\title{
A systematic review and economic model of switching from non-glycopeptide to glycopeptide antibiotic prophylaxis for surgery
}

G Cranny, R Elliott, H Weatherly,

D Chambers, N Hawkins, L Myers,

M Sculpher and A Eastwood

January 2008

Health Technology Assessment NHS R\&D HTA Programme www.hta.ac.uk

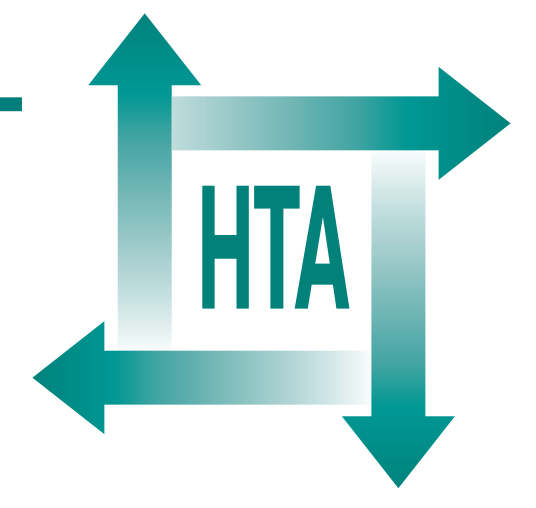




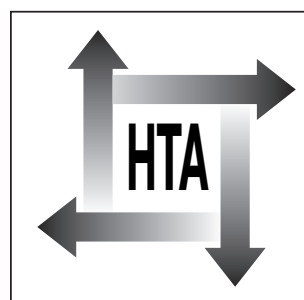

INAHTA

How to obtain copies of this and other HTA Programme reports.

An electronic version of this publication, in Adobe Acrobat format, is available for downloading free of charge for personal use from the HTA website (http://www.hta.ac.uk). A fully searchable CD-ROM is also available (see below).

Printed copies of HTA monographs cost $£ 20$ each (post and packing free in the UK) to both public and private sector purchasers from our Despatch Agents.

Non-UK purchasers will have to pay a small fee for post and packing. For European countries the cost is $£ 2$ per monograph and for the rest of the world $£ 3$ per monograph.

You can order HTA monographs from our Despatch Agents:

- fax (with credit card or official purchase order)

- post (with credit card or official purchase order or cheque)

- phone during office hours (credit card only).

Additionally the HTA website allows you either to pay securely by credit card or to print out your order and then post or fax it.

\section{Contact details are as follows:}

HTA Despatch

Email: orders@hta.ac.uk

c/o Direct Mail Works Ltd

Tel: 02392492000

4 Oakwood Business Centre

Fax: 02392478555

Downley, HAVANT PO9 2NP, UK

Fax from outside the UK: +442392478555

$\mathrm{NHS}$ libraries can subscribe free of charge. Public libraries can subscribe at a very reduced cost of $\notin 100$ for each volume (normally comprising 30-40 titles). The commercial subscription rate is $£ 300$ per volume. Please see our website for details. Subscriptions can only be purchased for the current or forthcoming volume.

\section{Payment methods}

Paying by cheque

If you pay by cheque, the cheque must be in pounds sterling, made payable to Direct Mail Works Ltd and drawn on a bank with a UK address.

Paying by credit card

The following cards are accepted by phone, fax, post or via the website ordering pages: Delta, Eurocard, Mastercard, Solo, Switch and Visa. We advise against sending credit card details in a plain email.

Paying by official purchase order

You can post or fax these, but they must be from public bodies (i.e. NHS or universities) within the UK. We cannot at present accept purchase orders from commercial companies or from outside the UK.

\section{How do I get a copy of HTA on CD?}

Please use the form on the HTA website (www.hta.ac.uk/htacd.htm). Or contact Direct Mail Works (see contact details above) by email, post, fax or phone. HTA on CD is currently free of charge worldwide.

The website also provides information about the HTA Programme and lists the membership of the various committees. 


\title{
A systematic review and economic model of switching from non-glycopeptide to glycopeptide antibiotic prophylaxis for surgery
}

\author{
G Cranny, ${ }^{\prime}$ R Elliott, ${ }^{2} \mathrm{H}$ Weatherly, ${ }^{3}$ \\ D Chambers,' $N$ Hawkins, ${ }^{3}$ L Myers, \\ $M$ Sculpher ${ }^{3}$ and A Eastwood ${ }^{1 *}$
}

I Centre for Reviews and Dissemination, University of York, UK

${ }^{2}$ School of Pharmacy, University of Manchester, UK

${ }^{3}$ Centre for Health Economics, University of York, UK

* Corresponding author

Declared competing interests of authors: none

Published January 2008

This report should be referenced as follows:

Cranny G, Elliott R, Weatherly H, Chambers D, Hawkins N, Myers L, et al. A systematic review and economic model of switching from non-glycopeptide to glycopeptide antibiotic prophylaxis for surgery. Health Technol Assess 2008; I 2(I).

Health Technology Assessment is indexed and abstracted in Index Medicus/MEDLINE, Excerpta Medica/EMBASE and Science Citation Index Expanded (SciSearch ${ }^{\circledR}$ ) and Current Contents ${ }^{\circledR} /$ Clinical Medicine. 


\section{NIHR Health Technology Assessment Programme}

$\mathrm{T}$ The Health Technology Assessment (HTA) Programme, part of the National Institute for Health Research (NIHR), was set up in 1993. It produces high-quality research information on the effectiveness, costs and broader impact of health technologies for those who use, manage and provide care in the NHS. 'Health technologies' are broadly defined as all interventions used to promote health, prevent and treat disease, and improve rehabilitation and long-term care.

The research findings from the HTA Programme directly influence decision-making bodies such as the National Institute for Health and Clinical Excellence (NICE) and the National Screening Committee (NSC). HTA findings also help to improve the quality of clinical practice in the NHS indirectly in that they form a key component of the 'National Knowledge Service'.

The HTA Programme is needs-led in that it fills gaps in the evidence needed by the NHS. There are three routes to the start of projects.

First is the commissioned route. Suggestions for research are actively sought from people working in the NHS, the public and consumer groups and professional bodies such as royal colleges and NHS trusts. These suggestions are carefully prioritised by panels of independent experts (including NHS service users). The HTA Programme then commissions the research by competitive tender.

Secondly, the HTA Programme provides grants for clinical trials for researchers who identify research questions. These are assessed for importance to patients and the NHS, and scientific rigour.

Thirdly, through its Technology Assessment Report (TAR) call-off contract, the HTA Programme commissions bespoke reports, principally for NICE, but also for other policy-makers. TARs bring together evidence on the value of specific technologies.

Some HTA research projects, including TARs, may take only months, others need several years. They can cost from as little as $£ 40,000$ to over 11 million, and may involve synthesising existing evidence, undertaking a trial, or other research collecting new data to answer a research problem.

The final reports from HTA projects are peer-reviewed by a number of independent expert referees before publication in the widely read journal series Health Technology Assessment.

\section{Criteria for inclusion in the HTA journal series}

Reports are published in the HTA journal series if (1) they have resulted from work for the HTA Programme, and (2) they are of a sufficiently high scientific quality as assessed by the referees and editors. Reviews in Health Technology Assessment are termed 'systematic' when the account of the search, appraisal and synthesis methods (to minimise biases and random errors) would, in theory, permit the replication of the review by others.

The research reported in this issue of the journal was commissioned by the HTA Programme as project number 05/36/01. The contractual start date was in September 2005. The draft report began editorial review in May 2006 and was accepted for publication in April 2007. As the funder, by devising a commissioning brief, the HTA Programme specified the research question and study design. The authors have been wholly responsible for all data collection, analysis and interpretation, and for writing up their work. The HTA editors and publisher have tried to ensure the accuracy of the authors' report and would like to thank the referees for their constructive comments on the draft document. However, they do not accept liability for damages or losses arising from material published in this report.

The views expressed in this publication are those of the authors and not necessarily those of the HTA Programme or the Department of Health.

Editor-in-Chief:

Professor Tom Walley

Series Editors:

Dr Aileen Clarke, Dr Peter Davidson, Dr Chris Hyde,

Programme Managers:

Dr John Powell, Dr Rob Riemsma and Professor Ken Stein

Sarah Llewellyn Lloyd, Stephen Lemon, Kate Rodger,

Stephanie Russell and Pauline Swinburne

ISSN | 366-5278

\section{(c) Queen's Printer and Controller of HMSO 2008}

This monograph may be freely reproduced for the purposes of private research and study and may be included in professional journals provided that suitable acknowledgement is made and the reproduction is not associated with any form of advertising.

Applications for commercial reproduction should be addressed to: NCCHTA, Mailpoint 728, Boldrewood, University of Southampton, Southampton, SOI6 7PX, UK.

Published by Gray Publishing, Tunbridge Wells, Kent, on behalf of NCCHTA.

Printed on acid-free paper in the UK by St Edmundsbury Press Ltd, Bury St Edmunds, Suffolk. 


\title{
Abstract
}

\section{A systematic review and economic model of switching from non-glycopeptide to glycopeptide antibiotic prophylaxis for surgery}

\author{
G Cranny, ' R Elliott, ${ }^{2}$ H Weatherly, ${ }^{3}$ D Chambers, ' $N$ Hawkins, ${ }^{3}$ L Myers, ' \\ M Sculpher ${ }^{3}$ and A Eastwood ${ }^{* *}$
}

I Centre for Reviews and Dissemination, University of York, UK

2 School of Pharmacy, University of Manchester, UK

${ }^{3}$ Centre for Health Economics, University of York, UK

* Corresponding author

Objectives: To determine whether there is a level of methicillin-resistant Staphylococcus aureus (MRSA) prevalence at which a switch from non-glycopeptide to glycopeptide antibiotics for routine prophylaxis is indicated in surgical environments with a high risk of MRSA infection.

Data sources: Major electronic databases were searched up to September 2005.

Review methods: The effectiveness review included controlled clinical trials comparing a glycopeptide with an alternative antibiotic regimen that reported effectiveness and/or adverse events. Controlled observational studies were also included for adverse events. The cost-effectiveness review included economic evaluations comparing glycopeptide prophylaxis with any alternative comparator. Study validity was assessed using standard checklists. The supplementary economic reviews assessed evaluations of non-glycopeptide antibiotic prophylaxis; evaluations where antibiotic resistance is a problem; methods of modelling resistance in infectious diseases; and developing a conceptual framework. An indicative decision analytic model was developed to compare vancomycin with a cephalosporin and with a combination of vancomycin and cephalosporin, using hip arthroplasty as an exemplar. Available data on, for example, surgical site infection (SSI) rates, MRSA rates, effectiveness of the antibiotics, were incorporated into the model. Costs were estimated from the perspective of the NHS.

Results: The effectiveness review included 16 randomised controlled trials, with a further three studies included for adverse events only. There was no evidence that glycopeptides were more effective than non-glycopeptides in preventing SSIs. Most of the trials did not report either the baseline prevalence of MRSA at the participating surgical units or MRSA infections as an outcome. The cost-effectiveness review included five economic evaluations of glycopeptide prophylaxis. Only one study incorporated health-related quality of life and undertook a cost-utility analysis. None of the studies was undertaken in the UK and none explicitly modelled antibiotic resistance. The supplementary reviews provided few insights into how to assess costeffectiveness in the context of resistance. No studies modelled cost-effectiveness alongside epidemiological models of resistance. There was little information regarding the impact of surgical infections on costs post-discharge and patient quality of life. The lack of available clinical evidence limited the development of the cost-effectiveness model and meant that the modelling could only be indicative in nature. The model can be used to show the threshold baseline risk at which the use of vancomycin as prophylaxis might be cost-effective (the model did not include teicoplanin). The indicative model suggests that the baseline risk of MRSA can be fairly modest at below the national average and it would still appear cost-effective to use glycopeptide prophylaxis. The model indicates that the use of glycopeptides as a form of prophylaxis in addition to a treatment for MRSA infections is unlikely to decrease the total usage and hence reduce the risk of future problems with glycopeptide-resistant bacteria.

Conclusions: There is insufficient evidence to determine whether there is a threshold prevalence of MRSA at which switching from non-glycopeptide to glycopeptide antibiotic prophylaxis might be clinically effective and cost-effective. Future research needs to address the complexities of decision-making relating to 
the prevention of MRSA and infection control in general. Research including evidence synthesis and decision modelling comparing a full range of interventions for infection control, which extends to other infections, not just MRSA, is needed. A long-term research programme to predict the pattern of drug resistance and its implications for future costs and health is also needed. 


\section{Contents}

Glossary and list of abbreviations

\section{Executive summary}

I Background

2 Aims and objectives

3 Systematic review of effectiveness

Methods

Results

Discussion

Summary

4 Systematic review of cost-effectiveness

Methods

Results

Discussion

Summary

5 Supplementary economic reviews

Introduction

Economic evaluations of

non-glycopeptide antibiotic prophylaxis

for surgery

Economic evaluations of antibiotics

where antibiotic resistance is a

problem

Use of epidemiological and decision

analytic techniques to model antibiotic resistance

A conceptual framework for the

economic evaluation of policies

against MRSA

Conclusions

6 Economic model

Introduction

Methods
63

63

vii

$\mathrm{xi}$

1

5

7

7

8

25

26

27

27

27

33

33

35
Results ........................................ 68

Discussion ............................................. 72

7 Discussion ........................................... 75

Development of a model to guide surgical antibiotic prophylaxis prescribing ...................................... 76

Recommendations for practice ............... 76

Recommendations for research ............... 77

8 Conclusions …................................... 79

Acknowledgements ............................. 81

References ..................................... 83

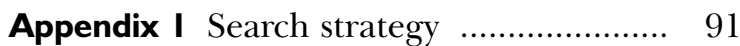

Appendix 2 Quality assessment checklists

Appendix 3 Studies excluded from the effectiveness review

Appendix 4 Data extraction tables: effectiveness review

Appendix 5 Data extraction tables: economic review

Appendix 6 Economic evaluation quality assessment

Health Technology Assessment reports published to date

Health Technology Assessment

Programme 



\section{8 \\ Glossary and list of abbreviations}

Technical terms and abbreviations are used throughout this report. The meaning is usually clear from the context, but a glossary is provided for the non-specialist reader. In some cases, usage differs in the

literature, but the term has a constant meaning throughout this review.

\section{Glossary}

Adverse event An abnormal or harmful effect caused by and attributable to exposure to an intervention, which is indicated by some result such as death, a physical symptom or visible illness. An effect may be classed as adverse if it causes functional or anatomical damage, causes irreversible change in the homeostasis of the organism or increases the susceptibility of the organism to other chemical or biological stress.

Blinding (synonym: masking) Keeping secret group assignment (e.g. to treatment or control) from the study participants or investigators. Blinding is used to protect against the possibility that knowledge of assignment may affect patient response to treatment, provider behaviours (performance bias) or outcome assessment (detection bias). Blinding is not always practical (e.g. when comparing surgery with drug treatment). The importance of blinding depends on how objective the outcome measure is; blinding is more important for less objective outcome measures such as pain or quality of life.

Concealment of allocation The process used to prevent foreknowledge of group assignment in a randomised controlled trial, which should be seen as distinct from blinding. The randomisation process should be administered by someone who is not responsible for recruiting participants, for example, a hospital pharmacy or a central office. Methods of assignment such as date of birth and case record numbers are open to manipulation. Adequate methods of allocation concealment include centralised randomisation schemes; randomisation schemes controlled by a pharmacy; numbered or coded containers in which capsules from identical-looking, numbered bottles are administered sequentially; on-site computer systems, where allocations are in a locked unreadable file; and sequentially numbered opaque, sealed envelopes.

Confidence interval (CI) Quantifies the uncertainty in measurement. Usually reported as $95 \%$ CI, i.e. the range of values within which one can be $95 \%$ sure that the true values for the whole population lie.

Cost-benefit analysis An attempt to give the consequences of the alternative interventions a monetary value. In this way, the consequences can be more easily compared with the costs of the intervention. This involves measuring individuals' 'willingness to pay' for given outcomes, and can be difficult.

Cost-consequence analysis Costs are reported separately from health effects.

Cost-effectiveness analysis The consequences of the alternatives are measured in natural units, such as years of life gained. The consequences are not given a monetary value.

Cost minimisation When two alternatives are found to have equal efficacy or outcomes (consequences). Therefore, the only difference between the two is cost. This is sometimes considered to be a subtype of cost-effectiveness analysis.

Cost-utility analysis The consequences of alternatives are measured in 'health state preferences', which are given a weighting score. In this type of analysis, different consequences are valued in comparison with each other, and the outcomes (e.g. life-years gained) are 


\section{Glossary continued}

adjusted by the weighting assigned. In this way, an attempt is made to value the quality of life associated with the outcome so that life-years gained become quality-adjusted life-years gained.

Discounting The process of converting future pounds sterling and future health effects to their present value.

Dominance The state when an intervention under study is both less costly and more effective than for the comparator(s).

Economic evaluation Comparative analysis of alternative course of action in terms of both their costs and effects.

Effectiveness The extent to which a specific intervention, when used under ordinary circumstances, does what it is intended to do.

Extended dominance The state when a strategy is both more costly and less effective than a linear combination of two other strategies with which it is mutually exclusive.

Hemiarthroplasty Arthroplasty where only the femur end of the hip joint is replaced with a prosthesis.

Incidence The number of new cases of a disease or event in a population during a specific period.

\section{Incremental cost-effectiveness ratio (ICER)}

An expression of the additional cost of health gain associated with an intervention relative to an appropriate comparator. Expressed as the difference in mean costs (relative to the comparator) divided by the difference in mean effects. Sometimes expressed with confidence intervals.

Intention-to-treat (ITT) An intention-to-treat analysis is one in which all the participants in a trial are analysed according to the intervention to which they were allocated, whether they received it or not. Intention-to-treat analyses are favoured in assessments of effectiveness as they mirror the non-compliance and treatment changes that are likely to occur when the intervention is used in practice and because of the risk of attrition bias when participants are excluded from the analysis.
Methodological quality (synonyms: validity, internal validity) The extent to which the design and conduct of a study are likely to have prevented systematic errors (bias). Variation in quality can explain variation in the results of studies included in a systematic review. More rigorously designed (better 'quality') trials are more likely to yield results that are closer to the 'truth'.

$p$-Value In the context of significance tests, the $p$-value represents the probability that a given difference is observed in a study sample, when such a difference does not exist in the relevant population. Small $p$-values indicate stronger evidence to reject the null hypothesis of no difference and a $p$-value of less than 0.05 indicates that a result is statistically significant.

Prevalence The measure of the proportion of people in a population who have some attribute or disease at a given point in time or during some time period.

Primary resistance This occurs when the initial infecting strain is resistant to standard treatment.

Quality-adjusted life-year (QALY) An index of health gain where survival duration is weighted or adjusted by the patient's quality of life during the survival period. QALYs have the advantage of incorporating changes in both quantity (mortality) and quality (morbidity) of life.

Randomised controlled trial (RCT) An experiment in which investigators randomly allocate eligible people into intervention groups to receive or not to receive one or more interventions that are being compared. The results are assessed by comparing outcomes in the treatment and control groups.

Relative risk (RR) (synonym: risk ratio) The ratio of risk in the intervention group to the risk in the control group. The risk (proportion, probability or rate) is the ratio of people with an event in a group to the total in the group. An $\mathrm{RR}=1$ indicates no difference between comparison groups. For undesirable outcomes, an $\mathrm{RR}<1$ indicates that the intervention was effective in reducing the risk of that outcome. 


\section{Glossary continued}

Secondary resistance This develops from an initially sensitive infecting strain in an individual during treatment.

Sensitivity analysis A mathematical method that examines uncertainty associated with parameter estimated into the analysis to test the robustness of the analysis findings. In oneway sensitivity analysis each parameter is varied individually, for multi-way analysis two or more parameters are varied at the same time, threshold analysis identifies the critical values above or below which the results of a study vary and analysis of extremes is used to examine the most pessimistic and the most optimistic scenarios. Finally, probabilistic sensitivity analysis attributes distributions of probabilities to uncertain variables that are incorporated within a model.

Systematic review A review of a clearly formulated question that uses systematic and explicit methods to identify, select and critically appraise relevant research, and to collect and analyse data from the studies that are included in the review.

Time trade-off (TTO) Measuring a health state by trading off life-years in a state of less than perfect health for a shorter life span in a state of perfect health.

Utility A measure of the strength of an individual's preference for a given health state or outcome. Utilities assign numerical values on a scale from 0 (death) to 1 (optimal or 'perfect' health), and provide a single number that summarises health.

Values An alternative measure of the strength of an individual's preference for a given health state or outcome. In contrast to utilities, values reflect preferences elicited in a risk-less context.

\section{List of abbreviations}

ACT artemisinin-based combination therapy

ADR

$\mathrm{AE}$

ASA

CABG adverse drug reaction

CCT controlled clinical trial

CDC Centers for Disease Control

CGE computable general equilibrium

CI confidence interval

CNS coagulase-negative staphylococci

CPS coagulase-positive staphylococci

CRD Centre for Reviews and Dissemination

CRPF chloroquine-resistant Plasmodium faciparum
DALY disability-adjusted life-year

DOT directly observed therapy

ENT ear, nose and throat

GART genotypic resistance screening

GDP gross domestic product

GISA glycopeptide intermediateresistance Staphylococcus aureus

GRSA glycopeptide-resistant Staphylococcus aureus

HAART highly active anti-retroviral therapy

HAI hospital-acquired infection

HIV human immunodeficiency virus

HRQoL health-related quality of life

ICER incremental cost-effectiveness ratio 


\section{List of abbreviations continued}

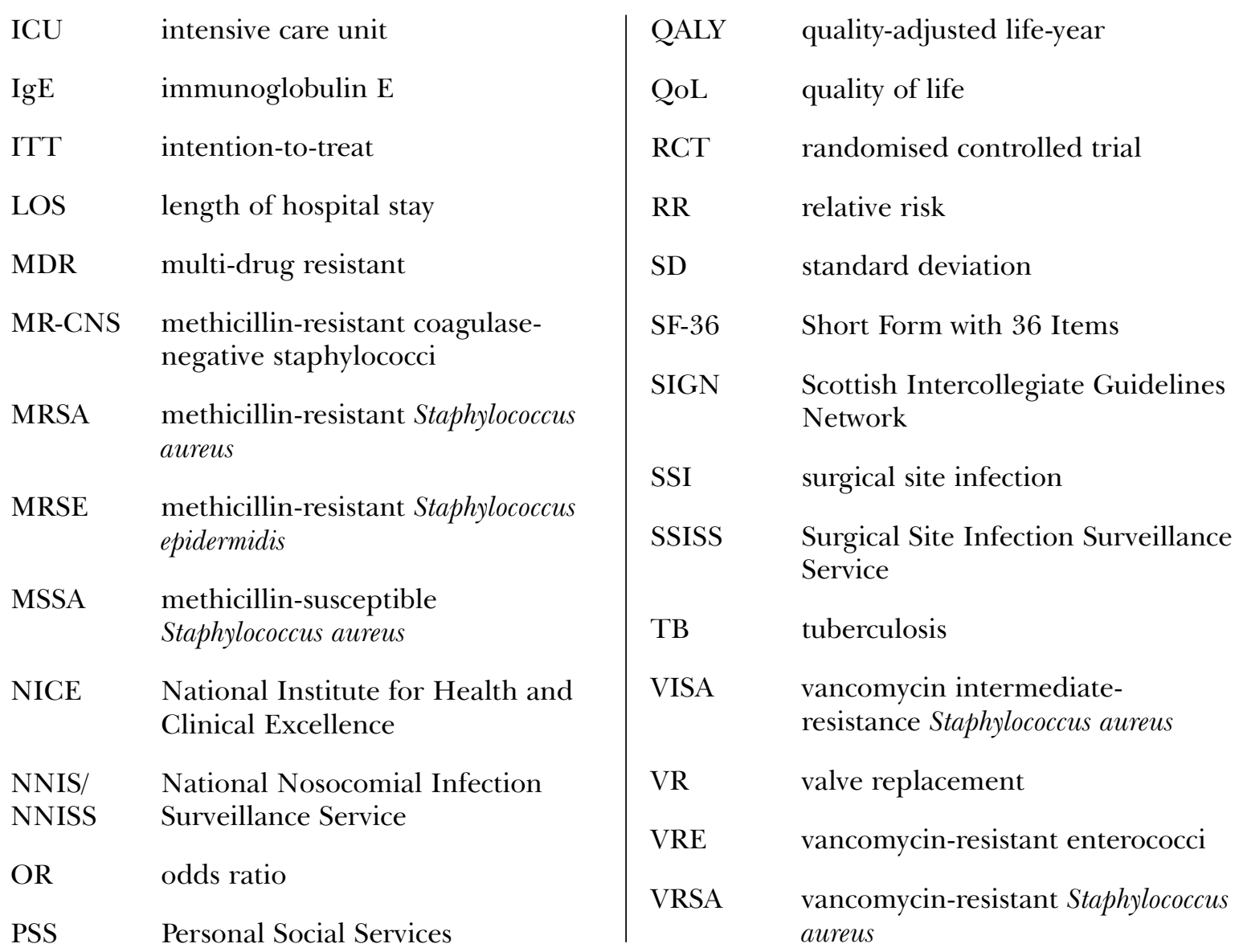

All abbreviations that have been used in this report are listed here unless the abbreviation is well known (e.g. NHS), or it has been used only once, or it is a non-standard abbreviation used only in figures/tables/appendices in which case the abbreviation is defined in the figure legend or at the end of the table. 


\section{8 \\ Executive summary}

\section{Background}

Surgical site infections (SSIs) are a major cause of morbidity and mortality in surgical patients.

Antibiotic prophylaxis is recommended when the risk of infection is high and/or the consequences of infection are likely to be severe. In recent years, the prevalence of antibiotic-resistant bacteria has increased markedly, methicillin-resistant Staphylococcus aureus (MRSA) being a cause of particular concern. Glycopeptide antibiotics (vancomycin and teicoplanin) are active against MRSA, but are normally reserved for the treatment of MRSA infections because of the perceived risk of selecting new resistant strains by increasing glycopeptide use. This project considers the implications of switching from nonglycopeptide to glycopeptide antibiotics for surgical prophylaxis.

\section{Objectives}

Our overall objective was to determine whether there is a level of MRSA prevalence at which a switch from non-glycopeptide to glycopeptide antibiotics for routine prophylaxis is indicated in surgical environments with a high risk of MRSA infection. We addressed this question by undertaking:

- a systematic review of the effectiveness of glycopeptide compared with non-glycopeptide antibiotic prophylaxis to determine whether there is evidence to guide antibiotic choice for surgical prophylaxis at different levels of MRSA prevalence

- a systematic review of published economic evaluations, to examine the cost-effectiveness of glycopeptide antibiotics compared with appropriate comparators

- a series of supplementary reviews, to support the modelling work and associated research recommendations

- a modelling approach to estimate the costeffectiveness of glycopeptide antibiotic prophylaxis relative to appropriate comparators, using orthopaedic surgery as an exemplar.

\section{Methods}

\section{Systematic reviews}

We searched 11 databases from 1990 to September 2005. Internet searches and searching of the reference lists of included papers were also performed. NHS EED, HEED and IDEAS were also searched for the cost-effectiveness review and modelling.

The effectiveness review included controlled clinical trials, comparing a glycopeptide with an alternative antibiotic regimen in adults undergoing surgical procedures where prophylaxis is recommended, that reported effectiveness and/or adverse events. Controlled observational studies were also included for adverse events. The cost-effectiveness review included economic evaluations comparing glycopeptide prophylaxis with any alternative comparator. Study validity was assessed using standard checklists.

\section{Supplementary reviews}

The supplementary economic reviews assessed evaluations of non-glycopeptide antibiotic prophylaxis; evaluations where antibiotic resistance is a problem; methods of modelling resistance in infectious diseases; and developing a conceptual framework.

\section{Economic modelling}

An indicative decision analytic model was developed to compare vancomycin with a cephalosporin and with a combination of vancomycin and cephalosporin, using hip arthroplasty as an exemplar. Available data on SSI rates, MRSA rates, effectiveness of the antibiotics in reducing infections and consequences of infection [impact on survival, length of hospital stay, healthrelated quality of life (HRQoL) and treatment intensity] were incorporated into the model. Costs were estimated from the perspective of the NHS.

\section{Results}

\section{Systematic reviews}

The effectiveness review included 16 randomised controlled trials, with a further three studies 
included for adverse events only. There was no evidence that glycopeptides were more effective than non-glycopeptides in preventing SSIs. Most of the trials did not report either the baseline prevalence of MRSA at the participating surgical units or MRSA infections as an outcome. The costeffectiveness review included five economic evaluations of glycopeptide prophylaxis. Only one study incorporated HRQoL and undertook a cost-utility analysis. None of the studies was undertaken in the UK, limiting the generalisability of the results to the UK, and none explicitly modelled antibiotic resistance.

\section{Supplementary reviews}

The supplementary reviews provided few insights into how to assess cost-effectiveness in the context of resistance. No studies modelled costeffectiveness alongside epidemiological models of resistance. In addition, there was little information regarding the impact of surgical infections on costs post-discharge and patient quality of life.

\section{Economic modelling}

The lack of available clinical evidence limited the development of the cost-effectiveness model and meant that the modelling could only be indicative in nature. Hip arthroplasty was chosen as an exemplar because it is a 'clean' procedure and patients are at high risk of MRSA. The model can be used to show the threshold baseline risk at which the use of vancomycin as prophylaxis might be cost-effective (the model did not include teicoplanin). The indicative model suggests that the baseline risk of MRSA (the average risk of MRSA infection in the population of patients undergoing hip arthroplasty in a given centre) can be fairly modest at below the national average and it would still appear cost-effective to use glycopeptide prophylaxis. However, this conclusion is reached in the absence of any modelling of the effect on resistance caused by increased glycopeptide use. The model indicates that, at all plausible baseline infection rates, the use of glycopeptides as a form of prophylaxis in addition to a treatment for MRSA infections is unlikely to decrease the total usage and hence reduce the risk of future problems with glycopeptide-resistant bacteria.

\section{Conclusions}

\section{Implications for healthcare}

There is insufficient evidence to determine whether there is a threshold prevalence of MRSA at which switching from non-glycopeptide to glycopeptide antibiotic prophylaxis might be clinically effective and cost-effective.

\section{Recommendations for research}

Future research needs to address the complexities of decision-making relating to the prevention of MRSA and infection control in general. Focusing on MRSA alone is too limited and the prophylactic use of glycopeptides is only one aspect of infection control.

Research including evidence synthesis and decision modelling comparing a full range of interventions for infection control, which extends to other infections, not just MRSA, is needed. A long-term research programme to predict the pattern of drug resistance and its implications for future costs and health is also needed. 


\section{Chapter I Background}

$\mathrm{H}$ ospital acquired infections (HAIs) are a major source of morbidity and mortality in the NHS and surgical site infection (SSI) is one of the most common types of HAI. ${ }^{1}$ Recent surveillance data from 102 English hospitals suggest that developing an SSI extends the patient's hospital stay by an average of 9 days, and up to 21 days for limb amputation, resulting in an additional cost ranging from $£ 959$ to $£ 6103 .^{2}$ When these SSIs are deep incisional or organ space infections, they also result in a substantial increase in risk of 30-day mortality, with odds ratios of 6.8 for vascular surgery and 2.5 for hip prosthesis surgery. ${ }^{2}$

Prophylactic administration of antibiotics in the pre- or perioperative period may reduce the risk of SSIs and other infections by inhibiting bacterial growth and adherence to prosthetic implants. Prophylaxis is not always indicated but is appropriate when the risk of infection is high and/or the consequences of any infection are likely to be severe (for example, in total hip

replacement). Guidelines produced by the Scottish Intercollegiate Guidelines Network (SIGN) ${ }^{3}$ (being updated at the time of writing this report) recommend prophylaxis for a wide range of surgical procedures classified as clean (no inflammation and no opening of the respiratory, alimentary or genitourinary tracts), cleancontaminated (respiratory, alimentary or genitourinary tracts are entered without significant spillage) or contaminated [acute inflammation or visible wound contamination (without pus) is present].

A range of different types of antibiotics with different modes of action is available for use in surgical prophylaxis. The cephalosporins have been most widely used and studied but regimens involving cephalosporins for surgical prophylaxis probably need to be reassessed for efficacy since vancomycin-resistant enterococci (VRE) and methicillin-resistant Staphylococcus aureus (MRSA) have become more prevalent in the UK. ${ }^{4}$ Other major antibiotic families include penicillins and other $\beta$-lactams, glycopeptides (vancomycin, teicoplanin), aminoglycosides (gentamicin, tobramicin, netilmicin and amikacin), macrolides (erythromycin and clarithromycin), quinolones/fluoroquinolones (ciprofloxacin, levofloxacin, norfloxacin and others), metronidazole, tetracyclines, sulfonamides, clindamycin, chloramphenicol and fusidic acid. Penicillins, glycopeptides, aminoglycosides and metronidazole are the principal agent groups used in surgical prophylaxis.

Most of the bacterial species causing SSIs can be categorised as either Gram-positive or Gramnegative, depending on how they respond to a Gram stain procedure. Bacteria are also either spherical (cocci) or rod-shaped (bacilli). These two criteria are easily visible by microscope and give rise to four easily identifiable subgroups of bacteria: Gram-positive cocci, Gram-positive bacilli, Gram-negative cocci and Gram-negative bacilli. Staphylococci may or may not have the enzyme coagulase, and their response to a test further divides them into 'coagulase-positive' or 'coagulase-negative' species.

Staphylococcus aureus (found on the skin and nares of about $30 \%$ of the population) is a coagulasepositive species and Staphylococcus epidermidis (found on the skin) is a coagulase-negative species (Figure 1). All species of staphylococci, both coagulase-positive and coagulase-negative, have developed resistance to many antibiotics. These are known as 'methicillin (or multi-) resistant'. This gives rise to MRSA and methicillin-resistant Staphylococcus epidermidis (MRSE). Primary resistance occurs when the initial infecting strain is resistant to standard treatment. Secondary resistance develops from an initially sensitive infecting strain in an individual during treatment.

The prevalence of MRSA has increased markedly over the last 10 years. Data on SSIs in hospitals in England indicate that during 1997-2002, 49\% of causal organisms identified were staphylococci, of which $81 \%$ were $S$. aureus. ${ }^{5}$ The majority of S. aureus strains $(63 \%)$ were MRSA. Although not a particularly virulent pathogen, MRSA can be difficult to treat and because of its concentration in hospitals, nursing homes and other care facilities it disproportionately affects the elderly and other vulnerable groups. The prevalence of MRSA infection in the community is also increasing, although cases mainly occur among 


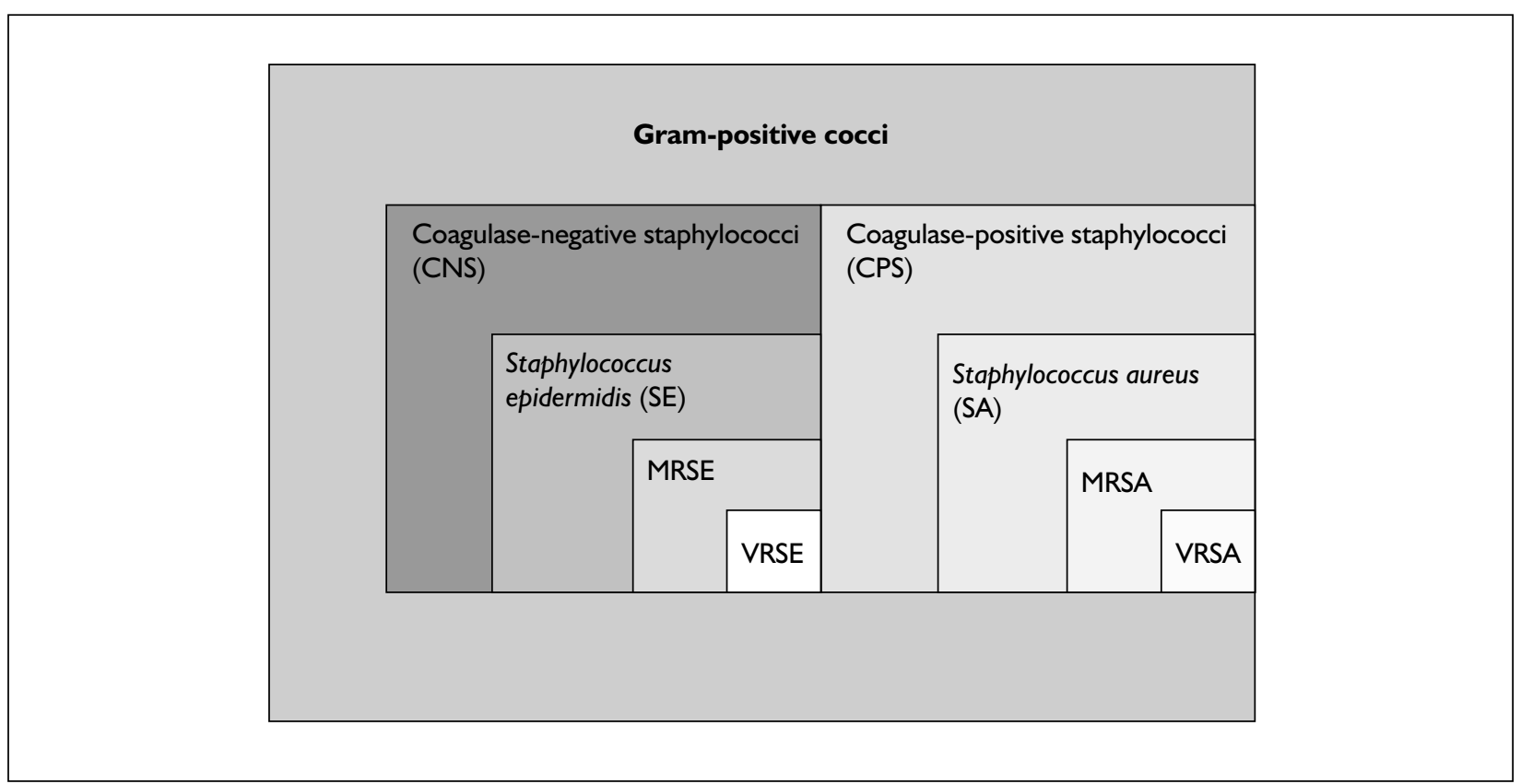

FIGURE I Gram-positive cocci families. VRSA, Vancomycin-resistant Staphylococcus aureus; VRSE, Vancomycin-resistant Staphylococcus epidermidis

people who have recently been in contact with the healthcare system. ${ }^{4}$

Glycopeptide antibiotics are active against Grampositive bacteria, including MRSA. Vancomycin was introduced during the 1950s and has generally been reserved for treatment of infections for which other antibiotics cannot be used because of patient sensitivity or bacterial resistance.

Teicoplanin has similar activity to vancomycin but has a longer duration of action. Other glycopeptide antibiotics are still under investigation

(oritavancin, ramoplanin) or are only used in animals (avoparcin). The SIGN guidelines do not recommend the use of glycopeptide antibiotics for prophylaxis because of lack of evidence of clinical benefit and concern that overuse of these drugs may increase the prevalence of vancomycinresistant bacteria. ${ }^{3}$ Similarly guidelines produced by the US Centers for Disease Control (CDC) in 1999 do not recommend vancomycin for routine prophylaxis, although they state that vancomycin may be the agent of choice in the presence of a cluster of MRSA infections or incisional SSIs caused by other methicillin-resistant bacteria. ${ }^{6}$

MRSA strains vary in their antibiotic resistance profiles. Methicillin-susceptible $S$. aureus (MSSA) may also possess acquired resistance to multiple antibiotics and be a source of new resistance combinations for MRSA. A survey of UK hospitals in 2004 found that $88 \%$ of MRSA isolates were
$93 \%$ were resistant to fluoroquinolones and $81 \%$ to macrolides and over $99 \%$ of strains were reported as fully susceptible to glycopeptide antibiotics (vancomycin and teicoplanin). ${ }^{4}$ However, vancomycin resistance has been detected in entercocci and subsequently in $S$. aureus $^{7,8}$ and MRSA strains with reduced susceptibility to teicoplanin have been identified in the $\mathrm{UK}^{9}$ and France. ${ }^{10}$ Depending on the mechanism of resistance, these strains are variably referred to as vancomycin-resistant. S. aureus (VRSA), glycopeptide-resistant $S$. aureus (GRSA), vancomycin intermediate-resistance $S$. aureus (VISA) and glycopeptide intermediate-resistance S. aureus (GISA).

From the viewpoint of the NHS and other national healthcare systems, SSIs caused by MRSA and other methicillin-resistant bacteria are primarily an issue in cardiac, vascular and orthopaedic surgery. Current recommendations for prophylaxis of infection in patients undergoing surgery are that glycopeptides should be limited to those with known MRSA infection or colonisation in order to limit selection for new glycopeptide-resistant strains. ${ }^{4}$ However, if prophylactic treatment with a single dose of a glycopeptide can prevent the development of an infection requiring longer treatment, possibly with higher doses, routine prophylaxis might have the effect of reducing overall antibiotic use. Some sources recommend the use of brief courses of vancomycin for prophylaxis in institutions where the prevalence of 
MRSA and MRSE is high, arguing that the benefits in terms of reducing the environmental pressure that promotes the development of vancomycin resistance and reducing the risk of superinfections caused by other bacteria and yeasts outweigh the risks. ${ }^{11}$ Although resistance to glycopeptides may be rare in the UK at present, the risk of new resistant strains emerging should be taken into account in determining policies for antibiotic use.

This study attempts to determine whether there is a threshold of MRSA prevalence at which switching to routine prophylaxis with a glycopeptide-based antibiotic regimen might be clinically effective and cost-effective. The individual patient's risk of developing an MRSA infection is affected by a range of policies, for example screening, patient isolation and prevention of transmission by healthcare staff. Screening for MRSA colonisation/infection and if necessary eradicating the organism before surgery have particular relevance to the risk of SSI caused by MRSA. The broader aspects of infection control that inform the perioperative and postoperative prevention and treatment of SSI will be dealt with in guidelines from the National Institute for Health and Clinical Excellence (NICE) that were under development at the time of writing this report (http://www.nice.org.uk/page.aspx?o=299840). Antibiotic prophylaxis was excluded from the scope of these guidelines but an update of the SIGN guidelines ${ }^{3}$ is due to be published in 2007. 



\section{Chapter 2}

\section{Aims and objectives}

$\mathrm{T}$ he overall objective of the project was to determine whether there is a level of MRSA prevalence at which a switch from nonglycopeptide to glycopeptide antibiotics for routine prophylaxis is indicated in surgical environments with a high risk of MRSA infection. We attempted to answer this question using a number of different approaches:

1. A systematic review of the effects of glycopeptide antibiotics compared with nonglycopeptide antibiotics on MRSA infection, overall infections, other morbidity and mortality, adverse events and occurrence and transmission of glycopeptide resistance in patients undergoing surgical procedures with a high risk of SSI or other postoperative infections. The objective of this review was to determine whether there is evidence from controlled clinical trials to guide antibiotic choice for surgical prophylaxis at different levels of MRSA prevalence.

2. A systematic review of published economic evaluations, to examine the cost-effectiveness of glycopeptide antibiotics compared with appropriate comparators.

3. A series of supplementary reviews, to support the modelling work and associated research recommendations. These involved reviews of: economic evaluations of non-glycopeptide prophylaxis for surgery; economic evaluations assessing cost-effectiveness in areas of infectious disease where drug resistance is an issue; use of epidemiological and decision analytic techniques to model antibiotic resistance; and conceptual papers which might contribute to a framework for the economic evaluation of policies against MRSA.

4. A modelling approach to estimate the costeffectiveness of glycopeptide antibiotic prophylaxis relative to appropriate comparators. In view of the range of different types of surgery and the time and resources available, we decided to focus on orthopaedic surgery (hip arthroplasty) as an exemplar of surgery where antibiotic prophylaxis is strongly recommended, and where there is evidence of its effectiveness from clinical trials. ${ }^{3}$ Our objective was to develop a model that incorporated the effects of different treatment strategies on the risk of infection and that included patient, environmental and procedural variables related to the risk of MRSA infection. An additional objective was to seek to incorporate into the model evidence on the occurrence and transmission of glycopeptide-resistant organisms. 



\section{Chapter 3}

\section{Systematic review of effectiveness}

\section{Methods}

This systematic review aimed to establish the effectiveness of glycopeptide antibiotic prophylaxis compared with any other antibiotic prophylaxis, and to summarise adverse events related to the use of glycopeptide prophylaxis. It was undertaken following the guidelines for undertaking systematic review produced by the Centre for Reviews and Dissemination (CRD). ${ }^{12}$

\section{Search strategy}

We searched the following databases from 1990 to September 2005: MEDLINE, EMBASE, CINAHL, CENTRAL, Science Citation Index and BIOSIS. The cut-off date of 1990 was chosen after discussion with clinical experts as MRSA was unlikely to have been reported in trials published before 1990 .

The search strategies used for all databases are presented in Appendix 1. In addition, information on studies in progress, unpublished research or research reported in the grey literature was sought by searching the following databases: ISI Proceedings; Science and Technology Edition; Inside Conferences; National Research Register; metaRegister of Controlled Trials; and the National Technical Information Service. No language restrictions were applied.

Internet searches were also carried out using the specialist search engine OMNI

(http://www.omni.ac.uk) and the meta-search engine Copernic (http://www.copernic.com).

Attempts to identify further studies were made by examining the reference lists of all retrieved articles.

\section{Study selection}

Study selection was a two-stage process. Initially, the titles and abstracts of items retrieved by the literature search were screened for relevance by two reviewers independently. Full copies of all potentially relevant papers were obtained. These were then assessed for inclusion by two reviewers. Disagreements were resolved by discussion, with referral to a third reviewer if necessary.

\section{Inclusion criteria}

In order to be included in the review a study had to meet all of the following criteria.

\section{Study design}

- Effectiveness: controlled clinical trials (CCTs) (randomised or quasi-randomised) investigating the use of glycopeptide antibiotics for prophylaxis compared with any alternative prophylactic antibiotic regimen.

- Adverse events: CCTs (randomised or quasirandomised) comparing a prophylactic glycopeptide regimen with any alternative prophylactic antibiotic regimen. Controlled observational studies of a glycopeptide antibiotic regimen, compared with any alternative antibiotic regimen, when used for surgical prophylaxis.

\section{Participants}

Participants were all adult patients (as defined by the studies) undergoing surgical procedures.

\section{Types of surgery}

The following surgical procedures had been used (both traditional and minimally invasive surgery), where antibiotic prophylaxis is recommended by the SIGN guidelines: ${ }^{3}$

- Clean-contaminated surgery: procedures with a high risk of bacterial contamination where the respiratory, gastrointestinal, urinary or genital tracts are opened.

- Clean surgery: procedures with a low risk of contamination where none of the above viscera are opened, but which require prophylaxis because serious complications could result from an infection. Cardiac, vascular and any procedures involving an implant (orthopaedic or vascular) are included in this category.

Studies of contaminated procedures (involving major breaks in sterile technique, significant spillage from the gastrointestinal tract, fresh open wounds or acute inflammation), dirty procedures (involving pre-existing infection or perforated viscera), infections as indication for surgery (appendicitis, cholecystitis, diverticulitis, salpingitis) and where further surgery was required 
after or because of a surgical site infection were excluded from the review.

\section{Interventions}

The intervention of interest was pre- or intraoperative administration of glycopeptide antibiotics (vancomycin, teicoplanin, ramoplanin and decaplanin) compared with any alternative antibiotic regimen. Postoperative administration was excluded. All routes of administration were considered. Multiple doses of antibiotic, continuing after surgery is complete, were considered provided that the initial dose was administered either before or during surgery. Both monotherapy and multiple drug regimens were eligible.

\section{Outcome measures}

Effectiveness

- Primary outcomes: the occurrence of an SSI, MRSA infection, any other infection (e.g. sepsis or bacteraemia) and mortality. SSIs were categorised as superficial, deep or organ space, where reported. The primary period was any infection that occurred within 30 days of surgery, although late infections occurring after 1 month from surgery were also reported.

- Secondary outcomes: length of postoperative hospital stay, rehospitalisation, reoperation, morbidity or disability and adverse events (AEs).

\section{Adverse events}

Any reported AEs considered to be related to the antibiotic prophylaxis.

\section{Data extraction}

Data were extracted by one reviewer and checked for accuracy and consistency by a second reviewer. Disagreements were resolved by discussion, with referral to a third reviewer if necessary.

The following information was extracted: study details and aims, study population, surgery details, details of the interventions (glycopeptide and comparator(s)), results (primary and secondary outcomes, organism causing infection, AEs) and study conclusions.

For studies included for AEs only, study, participant and surgery details were extracted as for the CCTs. Details of the glycopeptide regimen and full details of any AEs, their severity, relationship to the antibiotic and numbers of patients affected were extracted.

\section{Quality assessment}

randomisation, allocation concealment, similarity of treatment groups at baseline, specification of eligibility criteria, blinding (of outcome assessors and patients), intention-to-treat (ITT) analysis, sample size calculation and reporting of withdrawals (see Appendix 2 for quality checklists). The planned quality assessment of controlled observational studies was not performed as the only observational study included was published as an abstract. Quality assessments were carried out independently by two reviewers. Any disagreements were resolved by discussion, with referral to a third reviewer if necessary.

\section{Data analysis}

For each dichotomous outcome, the numbers of patients experiencing the outcome were extracted for each treatment group. The relative risk (RR) and $95 \%$ confidence interval (CI) were calculated for each trial on an ITT basis where possible. Continuous data were analysed by calculating the difference in means and corresponding $95 \%$ CI for each trial.

No statistical pooling was performed because of clinical heterogeneity between the studies due to differences in surgical procedures, comparator antibiotics, dose and timing of both glycopeptide and comparator regimens. The study results are presented in Forest plots and described in a narrative synthesis, grouped by outcome and surgical specialty.

Statistical analyses were performed using StatsDirect statistical software (www.statsdirect.com). This calculates 95\% CIs for the RR using the method of Koopman.

\section{Results}

\section{Identified studies}

The literature searches identified 11,689 references. These were screened for relevance and 65 were considered to be potentially relevant. These 65 articles were assessed using the predefined inclusion criteria. Figure 2 shows the flow of studies through the review process and the number of studies excluded. Full details of the excluded studies, together with the reasons for exclusion, are presented in Appendix 3.

\section{Nature of the evidence}

A total of 19 studies met the review inclusion criteria, with 16 studies providing results on clinical effectiveness and 12 studies providing 


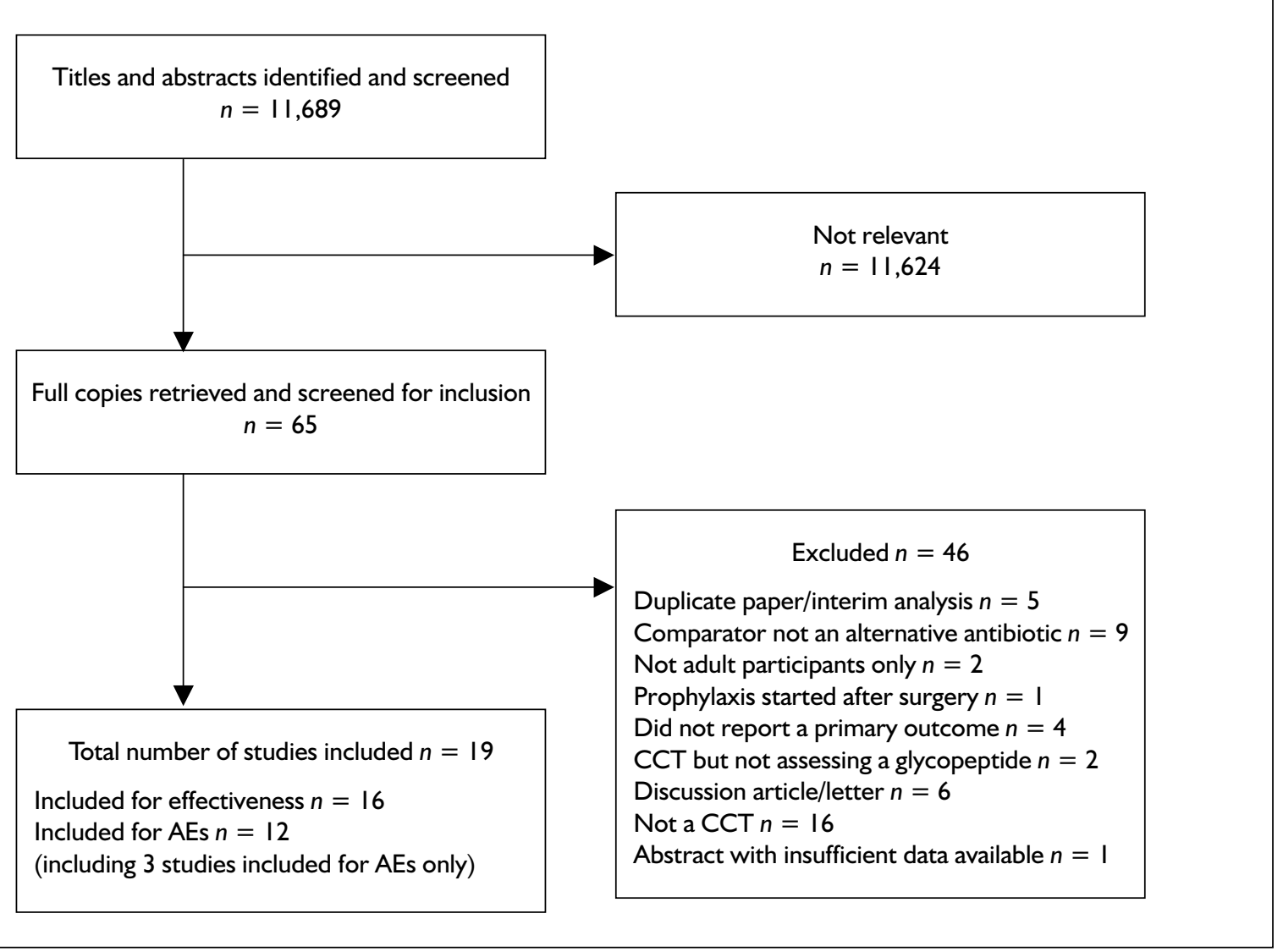

FIGURE 2 Flow chart of studies through the review process

results on AEs (three of these studies were included for AEs only). All studies, apart from one Spanish trial, ${ }^{13}$ were reported in English. Three studies ${ }^{14-16}$ were published as an abstract only.

The 16 studies included for effectiveness were all randomised controlled trials (RCTs). Five trials were in cardiac surgery; ${ }^{13,17-20}$ one trial included both cardiac and vascular procedures; ${ }^{21}$ three trials were in vascular surgery; ${ }^{16,22,23}$ five trials were in orthopaedic surgery; ${ }^{14,24-27}$ one trial was in neurosurgery; ${ }^{28}$ and one trial was in thoracic surgery. ${ }^{29}$ Most trials compared vancomycin or teicoplanin with a cephalosporin. The trial of cardiac and vascular surgery by Maki and colleagues $^{21}$ included two cephalosporin arms, cefazolin and cefamandole. As the trial reported comparisons of vancomycin versus the combined cephalosporin arms for the primary outcome of SSI, the results of the two cephalosporin arms have been combined in this review. Three trials compared a glycopeptide to an alternative glycopeptide regimen: one cardiac trial compared vancomycin with teicoplanin; ${ }^{13}$ one orthopaedic trial compared $400 \mathrm{mg}$ of teicoplanin given regionally with $800 \mathrm{mg}$ given systemically; ${ }^{27}$ and a trial of thoracic surgery in lung cancer patients compared long-term teicoplanin administration with short-term teicoplanin administration. ${ }^{29}$

An overview of the studies included for effectiveness detailing the surgical procedures, length of follow-up, prophylaxis regimens and outcomes reported is presented in Table 1 and an overview of the studies included for AE results only is presented in Table 2. Full details of all studies are presented in the data extraction tables in Appendix 4.

\section{Quality assessment}

The results of the quality assessment are summarised in Table 3, for studies included for effectiveness and those included for AEs only. Three studies ${ }^{14-16}$ were published in abstract form without enough information to allow a meaningful assessment of study quality and have been omitted from the table.

Of the 16 trials included for effectiveness, six used methods considered to produce true randomisation, including the use of computer programs, ${ }^{13,28}$ random number tables, ${ }^{20,25}$ permuted blocks ${ }^{22}$ and 


\begin{tabular}{|c|c|c|c|c|c|c|c|c|c|c|}
\hline 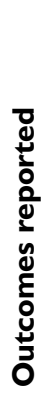 & 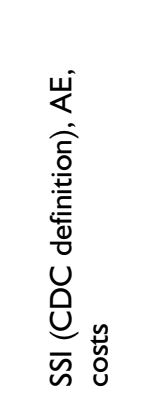 & 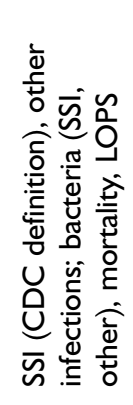 & 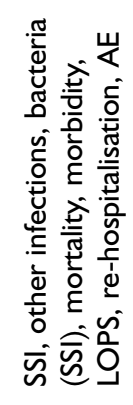 & 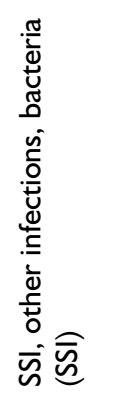 & 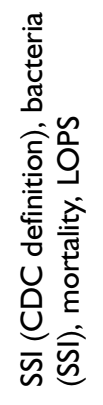 & 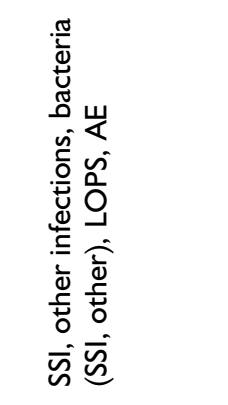 & 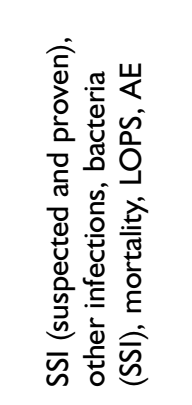 & 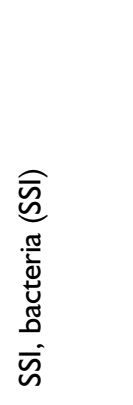 & 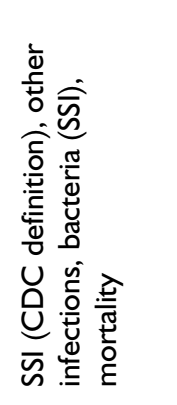 & \\
\hline 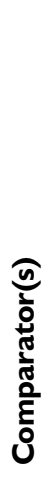 & 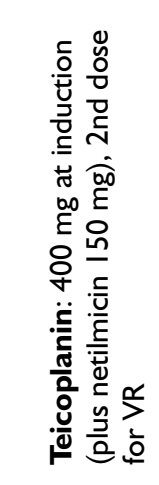 & 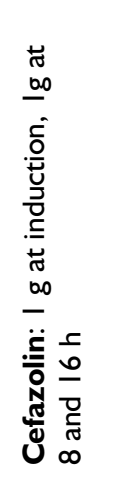 & 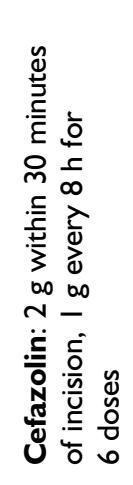 & 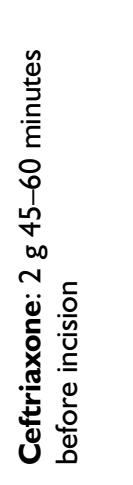 & 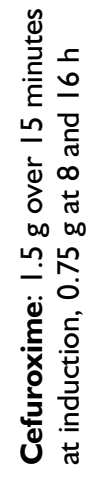 & 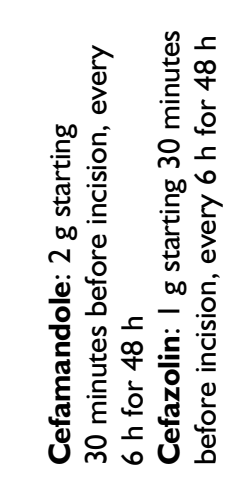 & 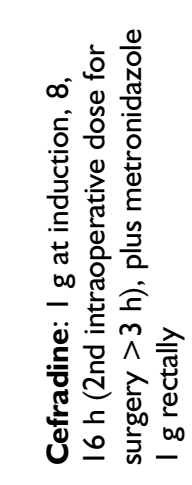 & 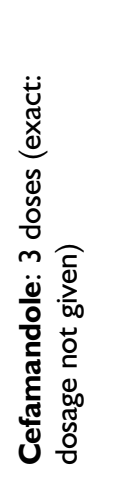 & 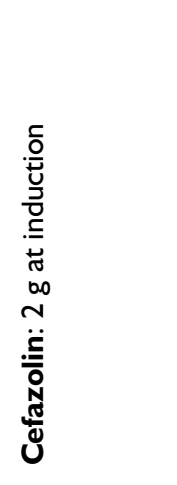 & \\
\hline 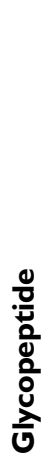 & 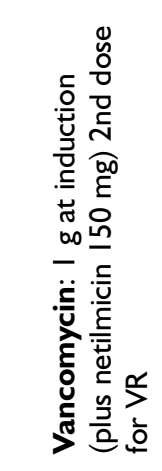 & 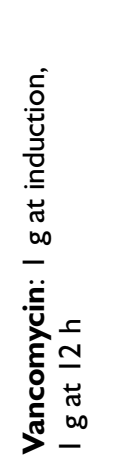 & 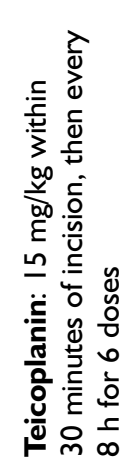 & 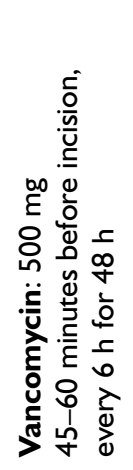 & 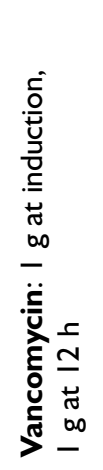 & 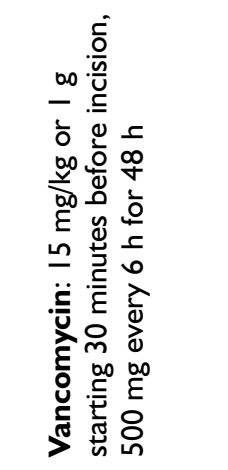 & 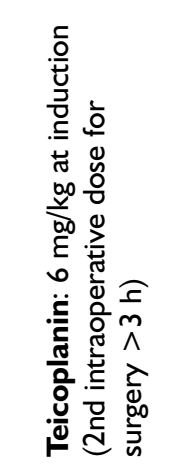 & 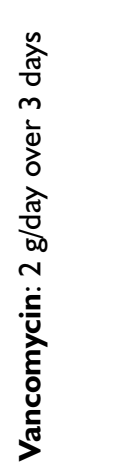 & 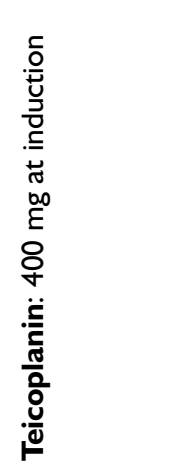 & \\
\hline $\begin{array}{l}\frac{0}{3} \\
\frac{1}{3} \\
\frac{0}{0} \\
4\end{array}$ & 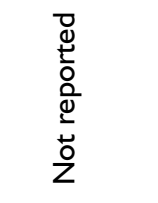 & 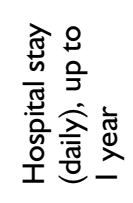 & 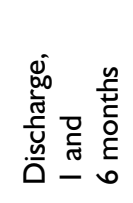 & 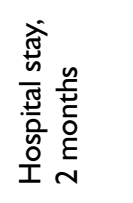 & 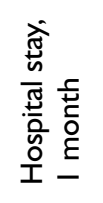 & 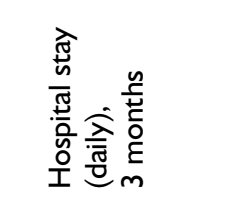 & 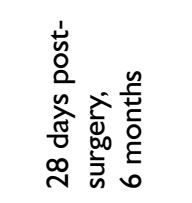 & 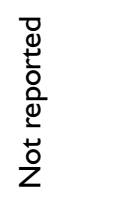 & 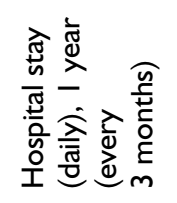 & \\
\hline$z$ & ৪ & $\stackrel{\widetilde{\tilde{O}}}{\underline{0}}$ & 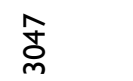 & ঃ্ & $\bar{\varnothing}$ & 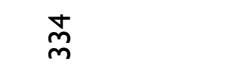 & $\underset{\sim}{\mathbb{N}}$ & ర్ & $\stackrel{\infty}{\sim}$ & \\
\hline 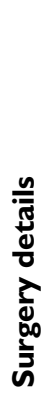 & 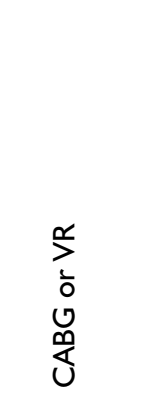 & 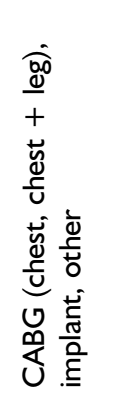 & 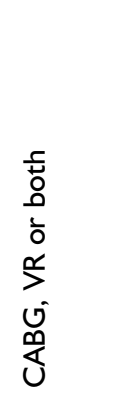 & 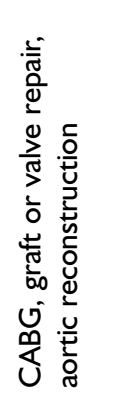 & 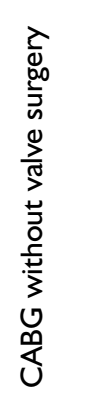 & 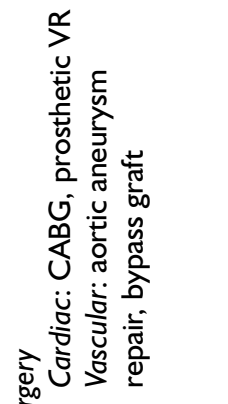 & 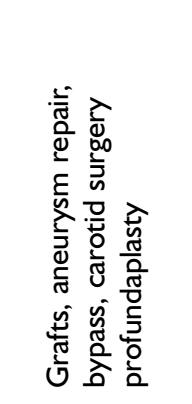 & 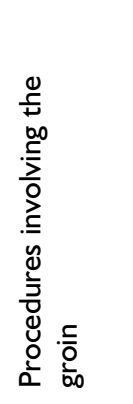 & 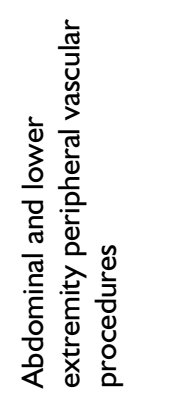 & \\
\hline $\begin{array}{l}\text { ते } \\
\text { मे }\end{array}$ & 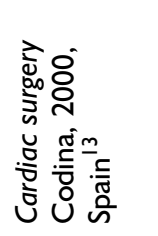 & 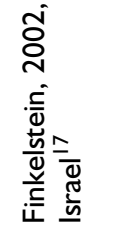 & 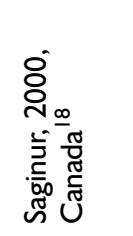 & 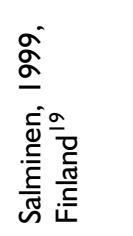 & 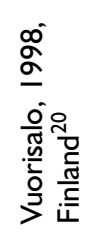 & 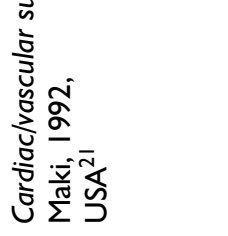 & 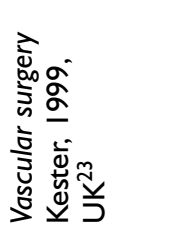 & 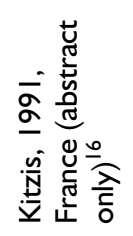 & 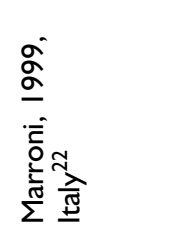 & \\
\hline
\end{tabular}




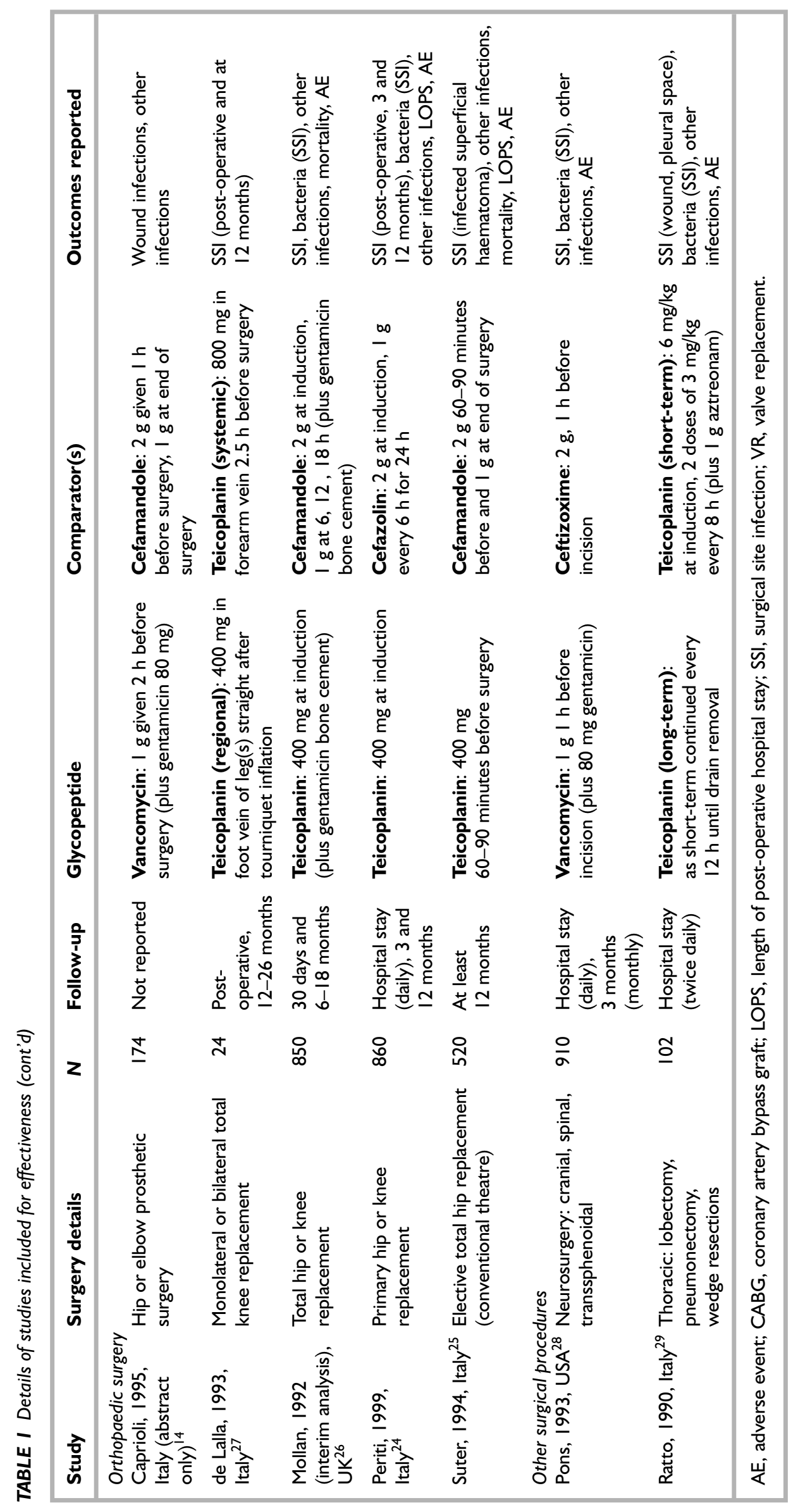




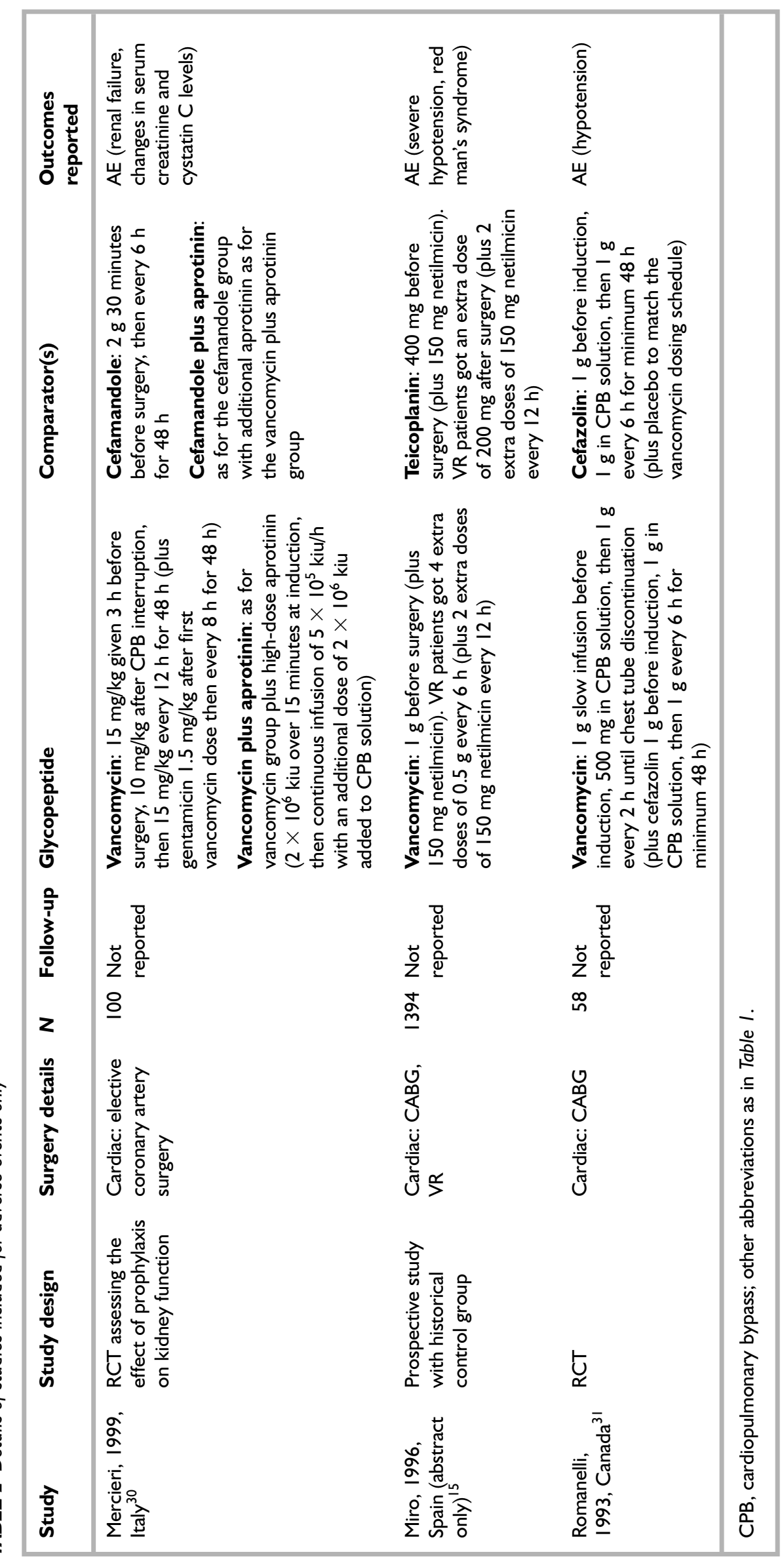




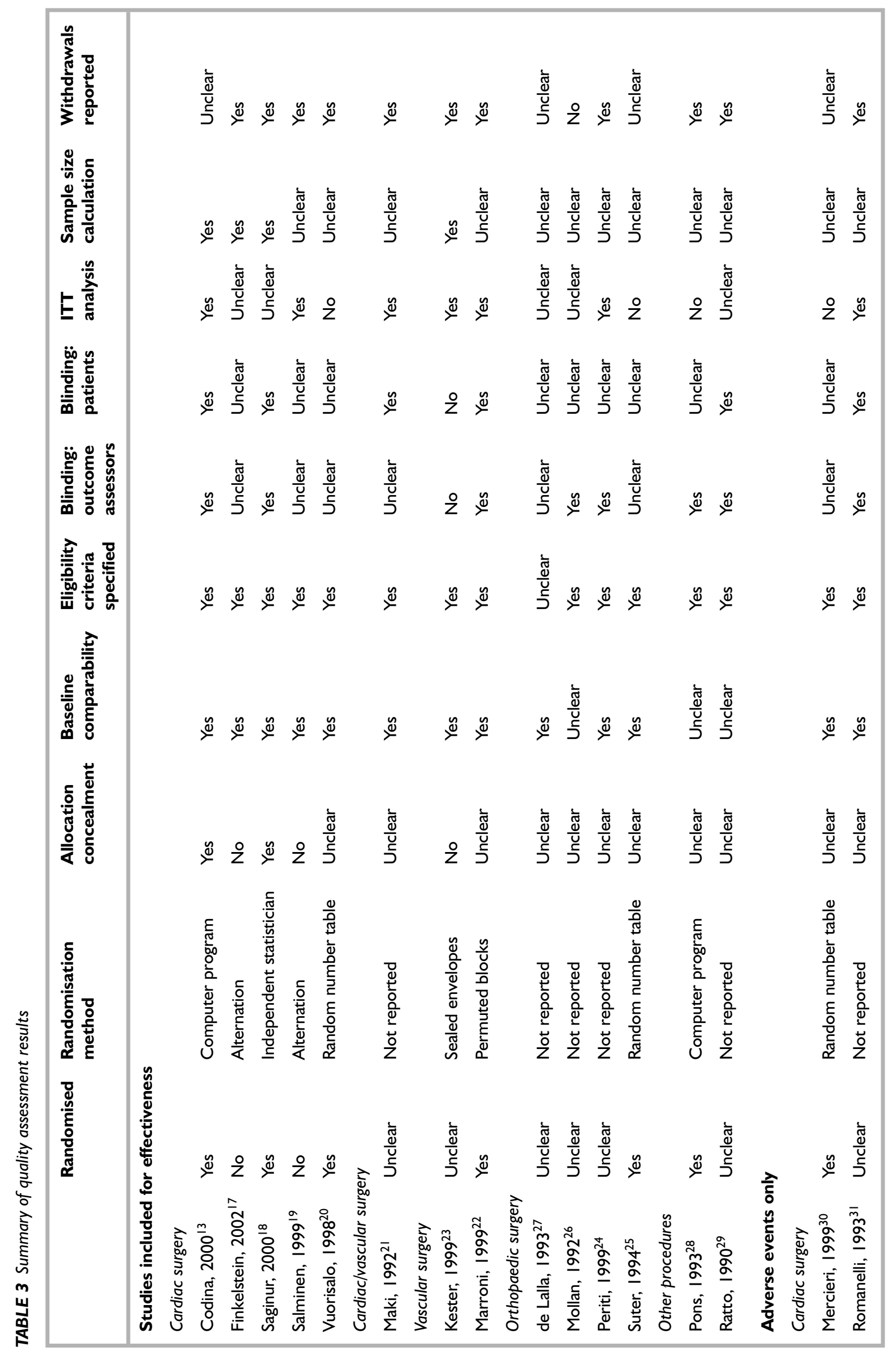


stratified randomisation by an independent statistician. ${ }^{18}$ Two trials used randomisation by alternation of birth date or social security number, ${ }^{17,19}$ which does not produce a genuinely random distribution between groups or conceal allocation of treatment. In the remaining trials, including four of the five in orthopaedic surgery, the method of randomisation was not reported. Concealment of treatment allocation was considered adequate in only two trials, both in cardiac surgery. ${ }^{13,18}$ Most trials reported their eligibility criteria and had treatment groups with similar characteristics at baseline.

Four trials reported blinding of both patients and outcome assessors ${ }^{13,18,22,29}$ and one was reported as an unblinded study; ${ }^{23}$ the other trials were unclear with respect to blinding of one or both groups. Six trials reported results on an ITT basis. ${ }^{13,19,21-24}$ A priori sample size calculations were reported for only four trials, ${ }^{13,17,18,23}$ three of which were in cardiac surgery. Withdrawals and drop-outs were reported for most trials. With two exceptions, ${ }^{25,26}$ trials that did not report withdrawals were focused on aspects other than effectiveness (cost-effectiveness ${ }^{13}$ or pharmacokinetics ${ }^{27}$ ).
Overall, the included trials showed a wide range of variation in methodological quality as presented in their published reports. The two trials with the best quality ratings were both in cardiac surgery, ${ }^{13,18}$ although one was a report of the economic analysis rather than the full trial results. ${ }^{13}$ One trial in vascular surgery also scored well on most quality criteria. ${ }^{22}$ Methodological aspects of the included trials in orthopaedic surgery were often poorly reported.

\section{Surgical site infection (SSI) Within $\mathbf{3 0}$ days of surgery}

Twelve trials reported the occurrence of an SSI within 30 days of surgery (or during postoperative hospitalisation if the trial did not define the timings of infections but the estimated overall duration of postoperative stay was approximately 30 days). ${ }^{17-26,28,29}$ The results are presented in Figure 3 and full details of all SSIs are presented in Table 4.

Only one trial found a statistically significant benefit of glycopeptide use. This was the trial of cardiac and vascular procedures by Maki and colleagues where fewer patients receiving vancomycin $(3.7 \%)$ had infections than patients

\section{Study}

Finkelstein $2002^{17}$

Saginur $2000^{18}$

Salminen $1999^{19}$

Vuorisalo $1998^{20}$

Maki $1992^{21}$

Kester $1999^{23}$

Marroni $1999^{22}$

Mollan $1992^{26}$

Periti $1999^{24}$

Suter $1994^{25}$

Pons $1993^{28}$

Ratto $1990^{29}$

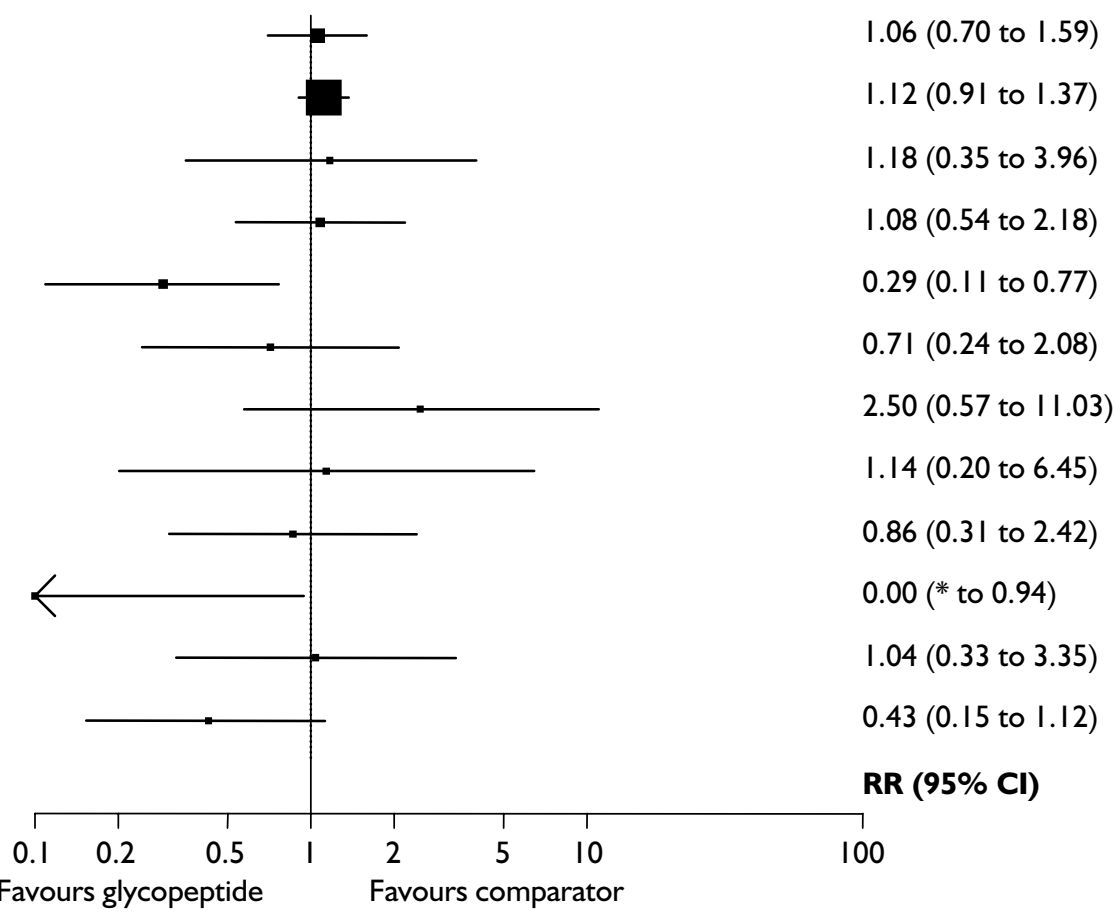


TABLE 4 Surgical site infections

\begin{tabular}{|c|c|c|c|c|c|}
\hline Surgery & Study & Glycopeptide & $n / N$ & Comparator & $n / N$ \\
\hline \multicolumn{6}{|c|}{ SSI within 30 days } \\
\hline \multirow[t]{4}{*}{ Cardiac } & Finkelstein, $2002^{17}$ & Vancomycin & $43 / 452$ & Cefazolin & $39 / 433$ \\
\hline & Saginur, $2000^{18}$ & Teicoplanin & $174 / 1518$ & Cefazolin & $155 / 1509$ \\
\hline & Salminen, $1999^{19}$ & Vancomycin & $5 / 103$ & Ceftriaxone & $4 / 97$ \\
\hline & Vuorisalo, $1998^{20}$ & Vancomycin & $15 / 440$ & Cefuroxime & $14 / 444$ \\
\hline Cardiac/vascular & Maki, $1992^{21}$ & Vancomycin & $4 / 107$ & $\begin{array}{l}\text { Cefazolin or } \\
\text { cefamandole }\end{array}$ & $29 / 227$ \\
\hline \multirow[t]{2}{*}{ Vascular } & Kester, $1999^{23}$ & Teicoplanin & $5 / 136$ & Cefradine & $7 / 136$ \\
\hline & Marroni, $1999^{22}$ & Teicoplanin & $5 / 119$ & Cefazolin & $2 / 119$ \\
\hline \multirow[t]{3}{*}{ Orthopaedic } & $\begin{array}{l}\text { Mollan, } 1992^{26} \\
\text { (infections occurring after 8-10 days) }\end{array}$ & Teicoplanin & $2 / 308$ & Cefamandole & $2 / 352$ \\
\hline & Periti, $1999^{24}$ & Teicoplanin & $6 / 422$ & Cefazolin & $7 / 424$ \\
\hline & Suter, $1994^{25}$ & Teicoplanin & $0 / 250$ & Cefamandole & $4 / 246$ \\
\hline Neurosurgery & Pons, $1993^{28}$ & Vancomycin & $5 / 404$ & Ceftizoxime & $5 / 422$ \\
\hline Thoracic & Ratto, $1990^{29}$ & $\begin{array}{l}\text { Teicoplanin } \\
\text { (long-term) }\end{array}$ & $4 / 25$ & $\begin{array}{l}\text { Teicoplanin } \\
\text { (short-term) }\end{array}$ & $9 / 24$ \\
\hline \multicolumn{6}{|c|}{ SSI after hospital discharge } \\
\hline \multirow[t]{2}{*}{ Cardiac } & Saginur, $2000^{18}$ (6 months post-surgery) & Teicoplanin & $32 / 1518$ & Cefazolin & $24 / 1509$ \\
\hline & $\begin{array}{l}\text { Vuorisalo, } 1998^{20} \text { (antibiotics prescribed } \\
\text { for suspected infections I month after } \\
\text { discharge) }\end{array}$ & Vancomycin & $80 / 440$ & Cefuroxime & $75 / 444$ \\
\hline \multirow[t]{2}{*}{ Vascular } & $\begin{array}{l}\text { Kester, } 1999^{23} \text { (bacteriologically proven } \\
\text { infections up to } 3 \text { months post-surgery) }\end{array}$ & Teicoplanin & $1 / 136$ & Cefradine & $1 / 136$ \\
\hline & $\begin{array}{l}\text { Marroni, } 1999^{22} \text { (graft infections up to } \\
3 \text { months post-surgery) }\end{array}$ & Teicoplanin & $2 / 119$ & Cefazolin & $0 / 119$ \\
\hline \multirow[t]{2}{*}{ Orthopaedic } & $\begin{array}{l}\text { Periti, } 1999^{24} \text { (deep wound infections } \\
3 \text { months post-surgery) }\end{array}$ & Teicoplanin & $3 / 375$ & Cefazolin & $3 / 364$ \\
\hline & $\begin{array}{l}\text { Periti, } 1999^{24} \text { (deep wound infections } \\
12 \text { months post-surgery) }\end{array}$ & Teicoplanin & $1 / 340$ & Cefazolin & $1 / 343$ \\
\hline \multicolumn{6}{|c|}{ No details of SSI timing reported } \\
\hline Orthopaedic & Caprioli, $1995^{14}$ & Vancomycin & $2 / 83$ & Cefamandole & $4 / 91$ \\
\hline Cardiac & Codina, $2000^{13}$ & Teicoplanin & $22 / 250$ & Vancomycin & $21 / 250$ \\
\hline Vascular & Kitzis, $1991^{16}$ & Vancomycin & $3 / 98$ & Cefamandole & $10 / 104$ \\
\hline
\end{tabular}

receiving either cefazolin or cefamandole $(12.8 \%)$ (RR $0.29,95 \%$ CI 0.11 to 0.77 ). ${ }^{21}$ The trial by Suter and colleagues of hip replacement surgery reported no SSIs for patients receiving teicoplanin and four $(1.6 \%)$ in patients receiving cefamandole (Fisher's exact test $p=0.059$, as reported by the authors; the estimated RR shown in Figure 3 has not been reported because of the incomplete $95 \%$ CI caused by zero values).$^{25}$ No statistically significant differences between glycopeptide and cephalosporin prophylaxis in preventing SSIs were found in the other trials.

\section{After hospital discharge}

Five trials provided results for late SSIs occurring after discharge (Figure 4). ${ }^{18,20,22-24}$ No statistically significant differences between glycopeptide and cephalosporin prophylaxis in preventing late infections were found in any of the trials. One vascular trial reported graft infections in two teicoplanin patients at 53 and 75 days after surgery (one was caused by MSSA) compared with no late infections in the cefazolin group. ${ }^{22}$ One orthopaedic trial reported details of infections occurring at 3 and 12 months, with there being no 


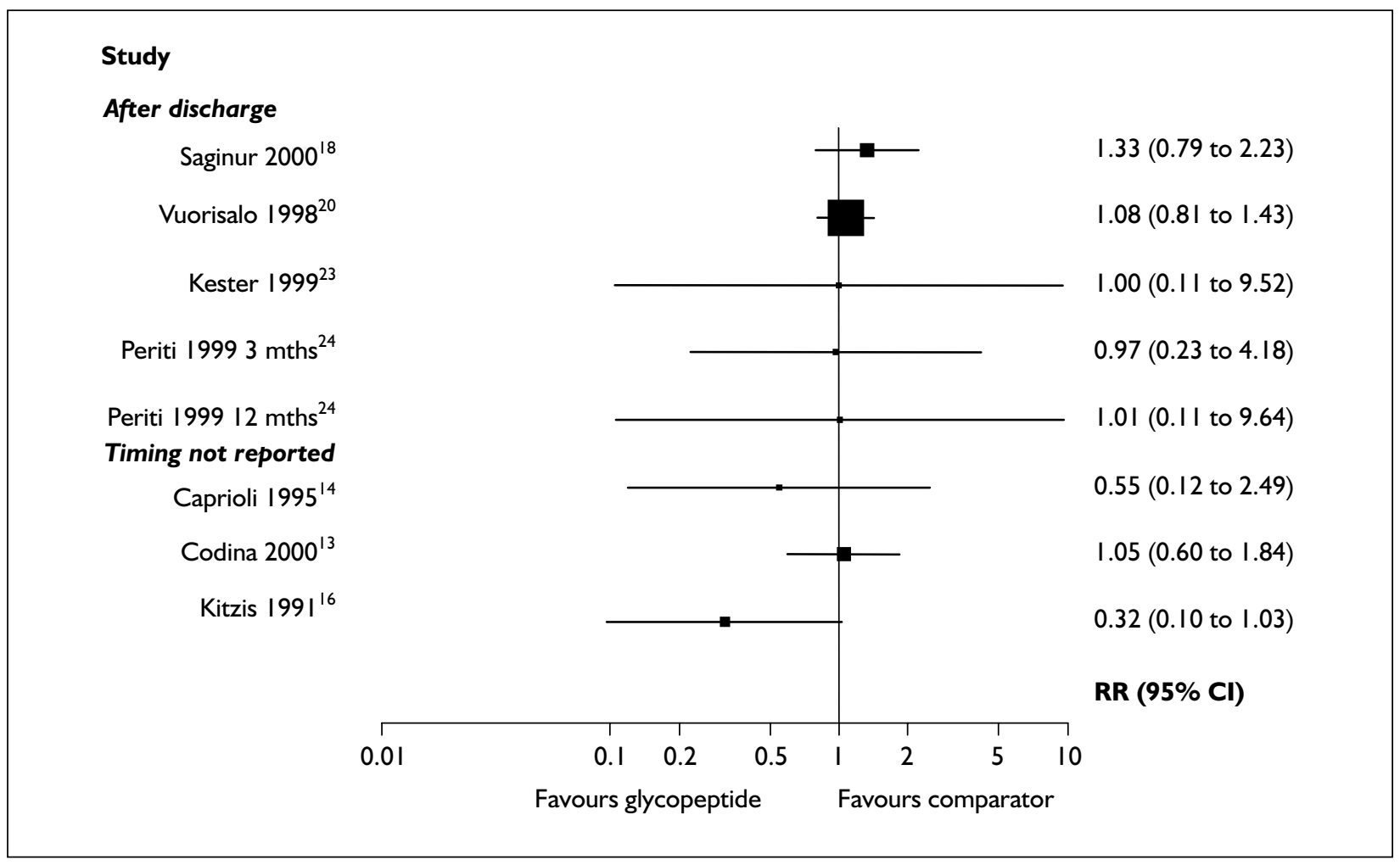

FIGURE 4 SSI after discharge

significant difference between teicoplanin and cefazolin (three infections at 3 months and one at 12 months in each group). ${ }^{24}$ Two other orthopaedic trials reported that no late infections occurred. ${ }^{25,27}$

Three trials ${ }^{13,14,16}$ did not provide information on when the SSI occurred and these are presented separately in Figure 4. No statistically significant differences between glycopeptide and cephalosporin prophylaxis in preventing infections were found in these three trials.

\section{Bacteria causing SSI MRSA}

Two trials reported the numbers of SSIs caused by MRSA. ${ }^{16,17}$ Three other trials reported that no MRSA infections occurred. ${ }^{19-21}$ The trial of cardiac surgery by Saginur and colleagues reported that $50 \%$ of S. epidermidis and $6.1 \%$ of S. aureus strains isolated during the trial were resistant to methicillin but did not report these results by treatment group. ${ }^{18}$ The remaining trials did not report whether they tested for MRSA. The results for infections caused by methicillin-resistant bacteria are presented in Figure 5 and Table 5. The trial by Finkelstein and colleagues found fewer cases of MRSA infections in patients receiving vancomycin compared with cefazolin [two $(0.4 \%)$ and seven (1.6\%), respectively], although this was not statistically significant. ${ }^{17}$ This trial was conducted in a cardiac surgical ward with a high prevalence of MRSA infections (incidence of new cases of infection or colonisation in 1995 and 1996 of $3 \%$ and $2.6 \%$, respectively). A trial of vascular surgery by Kitzis and colleagues reported no MRSA infections in vancomycin patients and four $(3.8 \%)$ in patients receiving cefamandole. ${ }^{16}$

In addition to the trial by Finkelstein and colleagues, ${ }^{17}$ a further three trials provided details of MRSA prevalence. One vascular trial reported that five (three CNS and two $S$. aureus) prosthetic vascular graft infections occurred in 298 procedures $(1.7 \%)$ in 1994 and three of these were methicillin resistant. ${ }^{22}$ One cardiac trial reported low prevalence but did not give any figures. ${ }^{18}$ Another cardiac trial also reported low prevalence (0-0.4\% from blood or pus specimens), with no new cases of MRSA occurring during the trial. ${ }^{20}$

\section{Other methicillin-resistant bacteria}

Three trials reported the numbers of SSIs caused by methicillin-resistant coagulase-negative staphylococci (MR-CNS). ${ }^{17,20,21}$ Patients receiving vancomycin [one $(0.2 \%)$ ] had significantly fewer MR-CNS infections compared with patients receiving cefuroxime [seven $(1.6 \%)$ in one trial of 


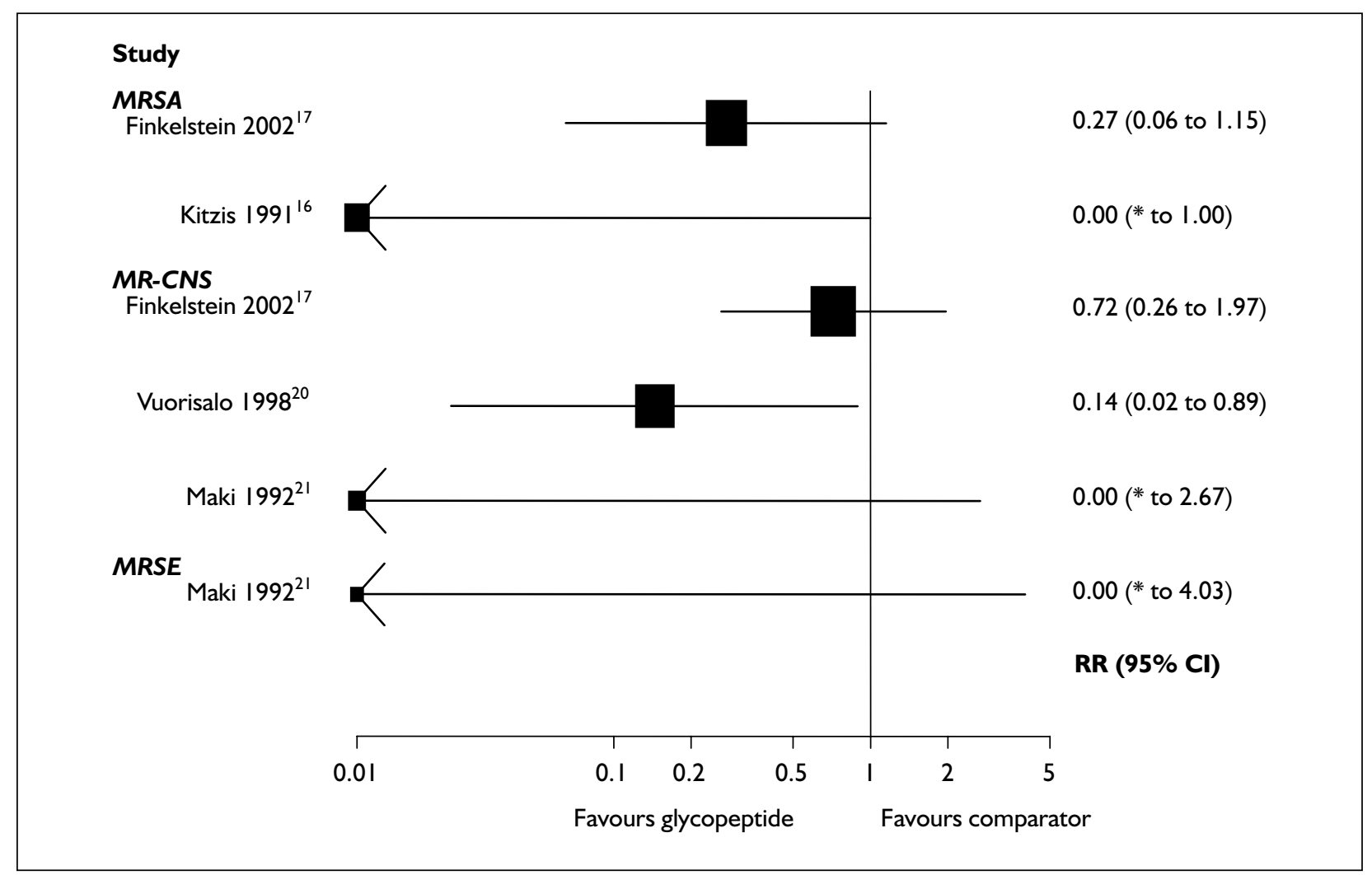

FIGURE 5 SSI caused by methicillin-resistant bacteria

cardiac surgery (RR $0.14,95 \%$ CI 0.02 to 0.89 ). ${ }^{20}$ No statistically significant differences between glycopeptide and cephalosporin prophylaxis were observed in the other two trials.

MRSE infections were reported in only one trial. One patient undergoing vascular surgery who received cefamandole in the trial by Maki and colleagues experienced a prosthetic graft infection caused by MRSE. ${ }^{21}$ No MRSE infections occurred in the vancomycin group.

\section{Glycopeptide-resistant bacteria}

Two trials reported testing for resistance to glycopeptides. ${ }^{18,21}$ The trial of cardiac surgery by Saginur and colleagues reported that no infections were resistant to teicoplanin, but $8 \%$ of the Grampositive and $34 \%$ of the Gram-negative infections were resistant to cefazolin. ${ }^{18}$ The trial of cardiac and vascular surgery by Maki and colleagues also reported that no infections were resistant to teicoplanin, but $20 \%$ of the Gram-positive and $60 \%$ of the Gram-negative infections were resistant to cefazolin or cefamandole. ${ }^{21}$

\section{Gram-positive bacteria}

Results for the total numbers of SSIs caused by Gram-positive bacteria (including all S.aureus, S. epidermidis and other CNS and CPS bacteria) are presented in Figure 6. No statistically significant differences between glycopeptide and cephalosporin prophylaxis were observed in any of the trials. Only the trial of thoracic surgery by Ratto, which compared long-term to short-term teicoplanin prophylaxis in lung cancer patients undergoing pulmonary resection, found a statistically significant reduction in infections caused by Gram-positive bacteria for long-term teicoplanin prophylaxis (RR $0.21,95 \%$ CI 0.06 to 0.76 ).$^{29}$

\section{Other bacteria}

Results for the numbers of SSIs caused by other bacteria are presented in Figure 7. This includes infections caused by Gram-negative bacteria, anaerobes and polymicrobial infections (mixed Gram-positive and -negative bacteria). No statistically significant differences between glycopeptide and cephalosporin prophylaxis were observed in any of the trials.

\section{Other infections}

Details of other infections remote from the surgical site are presented in Figures 8-10 and Table 6. Four trials reported the occurrence of bloodstream infections (Figure 8). ${ }^{17,18,21,22}$ No statistically significant differences between glycopeptide and cephalosporin prophylaxis were observed in any of the trials. 
TABLE 5 Bacteria causing SSI

\begin{tabular}{|c|c|c|c|c|c|}
\hline Surgery & Study & Glycopeptide & $n / N$ & Comparator & $n / N$ \\
\hline \multicolumn{6}{|l|}{ MRSA } \\
\hline Cardiac & Finkelstein, $2002^{17}$ & Vancomycin & $2 / 452$ & Cefazolin & $7 / 433$ \\
\hline Vascular & Kitzis, $1991^{16}$ & Vancomycin & $0 / 98$ & Cefamandole & $4 / 104$ \\
\hline \multicolumn{6}{|l|}{ MR-CNS } \\
\hline \multirow[t]{2}{*}{ Cardiac } & Finkelstein, $2002^{17}$ & Vancomycin & $6 / 452$ & Cefazolin & $8 / 433$ \\
\hline & Vuorisalo, $1998^{20}$ & Vancomycin & $1 / 440$ & Cefuroxime & $7 / 444$ \\
\hline Cardiac/vascular & Maki, $1992^{21}$ & Vancomycin & $0 / 107$ & $\begin{array}{l}\text { Cefamandole or } \\
\text { cefazolin }\end{array}$ & $3 / 227$ \\
\hline \multicolumn{6}{|l|}{ MRSE } \\
\hline Cardiac/vascular & Maki, 199221 & Vancomycin & $0 / 107$ & $\begin{array}{l}\text { Cefamandole or } \\
\text { cefazolin }\end{array}$ & $\mathrm{I} / 227$ \\
\hline \multicolumn{6}{|c|}{ Gram-positive (total: including S. aureus, S. epidermidis and other CNS and CPS) } \\
\hline Cardiac & Finkelstein, $2002^{17}$ & Vancomycin & $26 / 452$ & Cefazolin & $26 / 433$ \\
\hline & Saginur, $2000^{18}$ & Teicoplanin & $83 / 1518$ & Cefazolin & $60 / 1509$ \\
\hline & Salminen, $1999^{19}$ & Vancomycin & $3 / 103$ & Ceftriaxone & 2/97 \\
\hline & Vuorisalo, $1998^{20}$ & Vancomycin & $11 / 440$ & Cefuroxime & $14 / 444$ \\
\hline Cardiac/vascular & Maki, $1992^{21}$ & Vancomycin & $2 / 107$ & Cefazolin & $15 / 227$ \\
\hline \multirow[t]{3}{*}{ Vascular } & Kester, $1999^{23}$ & Teicoplanin & $2 / 136$ & Cefradine & $2 / 136$ \\
\hline & Marroni, $1999^{22}$ & Teicoplanin & $0 / 119$ & Cefazolin & $1 / 119$ \\
\hline & Kitzis, $1991^{16}$ & Vancomycin & $1 / 98$ & Cefamandole & $4 / 104$ \\
\hline \multirow[t]{3}{*}{ Orthopaedic } & Mollan, $1992^{26}$ & Teicoplanin & $2 / 308$ & Cefamandole & $2 / 352$ \\
\hline & Periti, $1999^{24}$ & Teicoplanin & $3 / 422$ & Cefazolin & $4 / 424$ \\
\hline & Suter, $1994^{25}$ & Teicoplanin & $0 / 250$ & Cefamandole & $4 / 246$ \\
\hline Neurosurgery & Pons, $1993^{28}$ & Vancomycin & $2 / 404$ & Ceftizoxime & $4 / 422$ \\
\hline Thoracic & Ratto, $1990^{29}$ & $\begin{array}{l}\text { Teicoplanin } \\
\text { (long-term) }\end{array}$ & $2 / 25$ & $\begin{array}{l}\text { Teicoplanin } \\
\text { (short-term) }\end{array}$ & $9 / 24$ \\
\hline \multicolumn{6}{|c|}{ Other bacteria (total: including Gram-negative, polymicrobial and anaerobic infections) } \\
\hline \multirow[t]{4}{*}{ Cardiac } & Finkelstein, $2002^{17}$ & Vancomycin & $21 / 452$ & Cefazolin & $20 / 433$ \\
\hline & Saginur, $2000^{18}$ & Teicoplanin & $21 / 1518$ & Cefazolin & $27 / 1509$ \\
\hline & Salminen, $1999^{19}$ & Vancomycin & $1 / 103$ & Ceftriaxone & $1 / 97$ \\
\hline & Vuorisalo, $1998^{20}$ & Vancomycin & $2 / 440$ & Cefuroxime & $0 / 444$ \\
\hline Cardiac/vascular & Maki, $1992^{21}$ & Vancomycin & $0 / 107$ & Cefazolin & $5 / 227$ \\
\hline \multirow[t]{3}{*}{ Vascular } & Kester, $1999^{23}$ & Teicoplanin & $4 / 136$ & Cefradine & $6 / 136$ \\
\hline & Marroni, $1999^{22}$ & Teicoplanin & $2 / 119$ & Cefazolin & $1 / 119$ \\
\hline & Kitzis, $\left.199\right|^{16}$ & Vancomycin & $2 / 98$ & Cefamandole & $6 / 104$ \\
\hline Orthopaedic & Periti, $1999^{24}$ & Teicoplanin & $1 / 422$ & Cefazolin & $2 / 424$ \\
\hline Thoracic & Ratto, $1990^{29}$ & $\begin{array}{l}\text { Teicoplanin } \\
\text { (long-term) }\end{array}$ & $2 / 25$ & $\begin{array}{l}\text { Teicoplanin } \\
\text { (short-term) }\end{array}$ & $0 / 24$ \\
\hline
\end{tabular}

Ten trials reported the occurrence of respiratory tract infections (Figure 9). ${ }^{14,18,19,21-25,28,29}$ The trial of cardiac surgery by Saginur and colleagues reported significantly more respiratory tract infections in the teicoplanin patients $(7.6 \%)$ than in the cefazolin patients $(4.7 \%)(\mathrm{RR} 1.62,95 \% \mathrm{CI}$ 1.22 to 2.16$).^{18}$

Eight trials reported the occurrence of urinary tract infections (Figure 10). ${ }^{14,18,19,21,22,24,25,28}$ The trial by Saginur and colleagues was again the only between a glycopeptide and a cephalosporin with more teicoplanin patients $(7.5 \%)$ than cefazolin patients (1.8\%) (RR 4.2, 95\% CI 2.79 to 6.33 ) experiencing a urinary tract infection. ${ }^{18}$

\section{Bacteria causing other infections}

In the trial of cardiac surgery by Finkelstein and colleagues, ${ }^{17}$ MRSA was isolated in the bloodstream infections of two $(0.4 \%)$ vancomycin patients and four $(0.9 \%)$ cefazolin patients, and MR-CNS was isolated in two $(0.4 \%)$ vancomycin patients and two $(0.5 \%)$ cefazolin patients. In the 


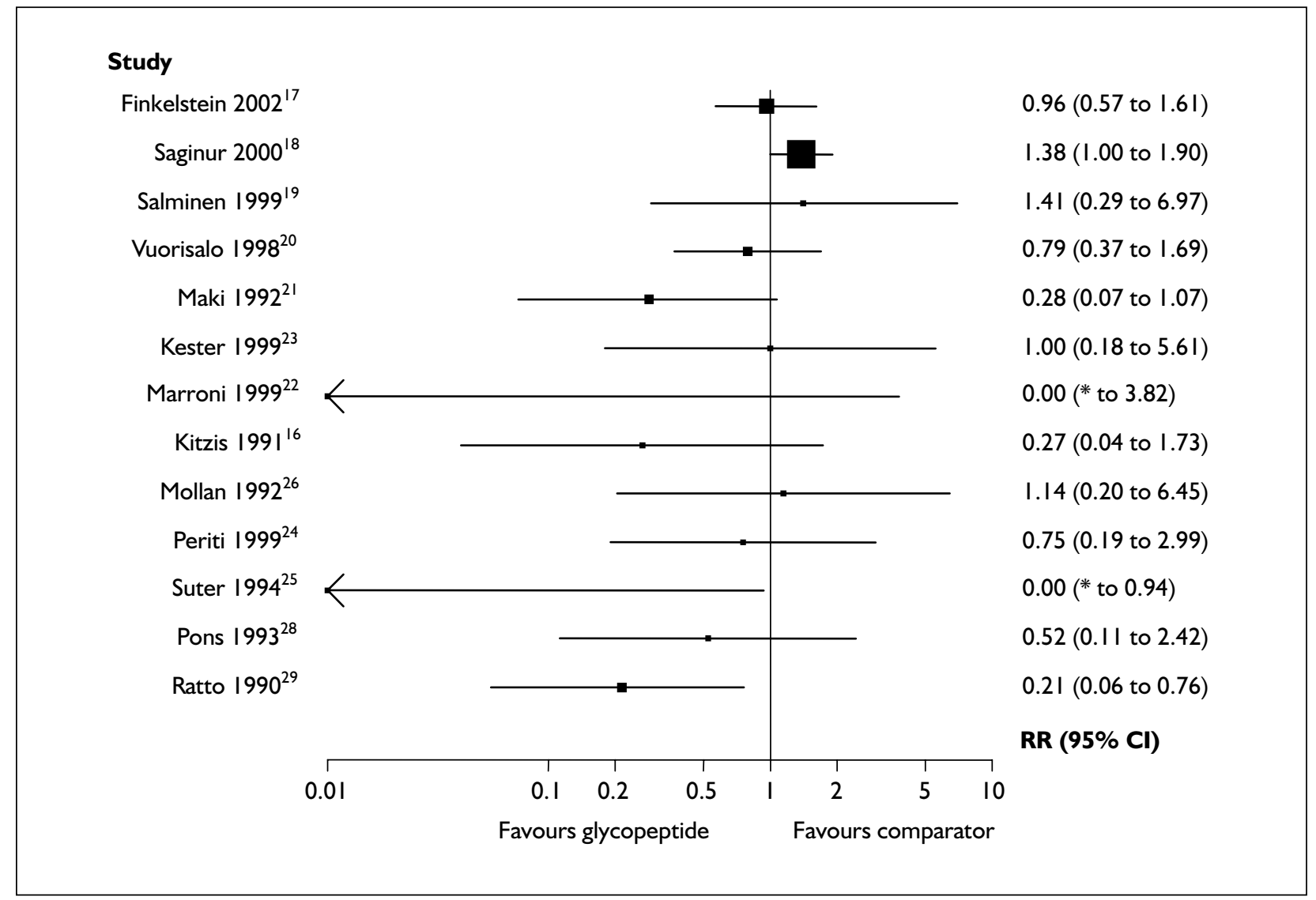

FIGURE 6 SSI caused by Gram-positive bacteria

\section{Study}

Finkelstein $2002^{17}$

Saginur $2000^{18}$

Salminen $1999^{19}$

Maki $1992^{21}$

Kester $1999^{23}$

Marroni $1999^{22}$

Kitzis $|99|^{16}$

Periti $1999^{24}$

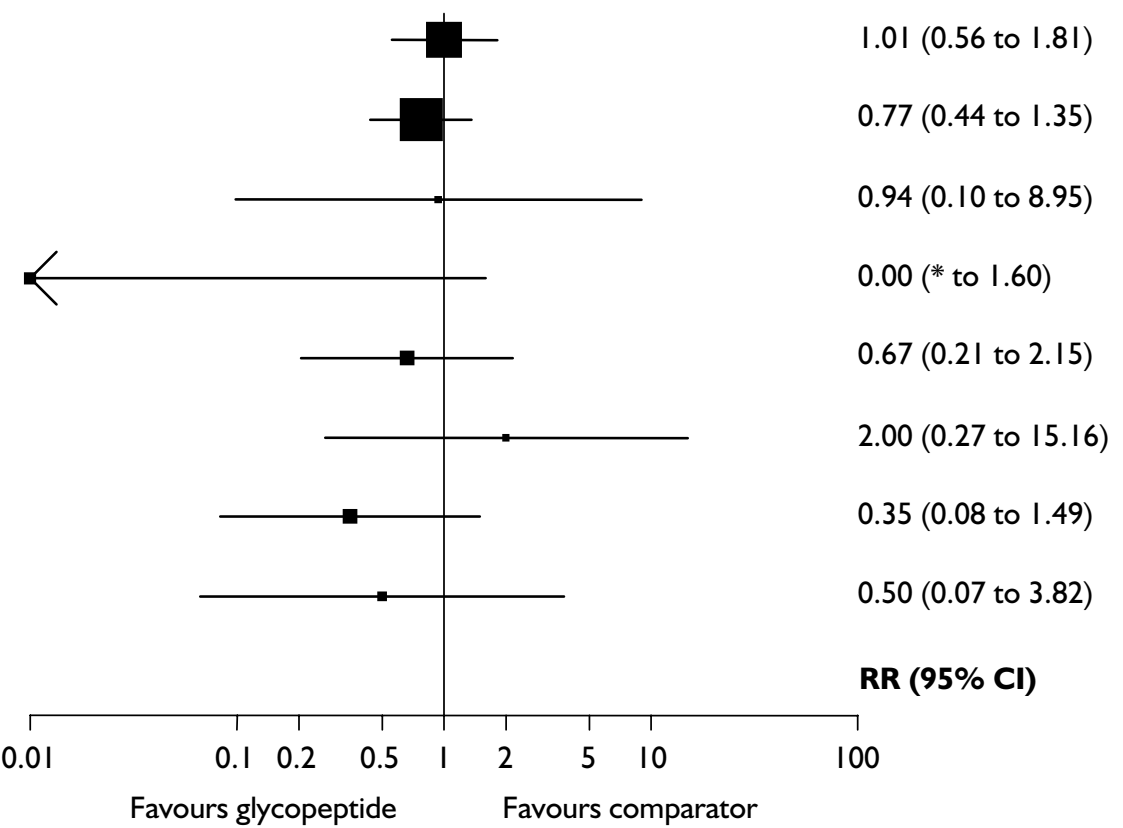




\section{Study}

Finkelstein $2002^{17}$

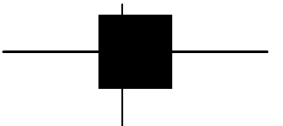

$1.06(0.58$ to 1.97$)$

Saginur $2000^{18}$

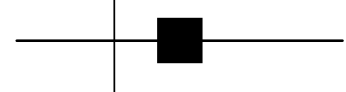

1.36 (0.64 to 2.89$)$

Maki $1992^{2}$

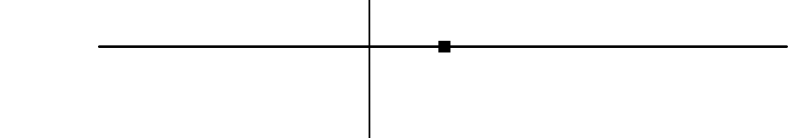

I.4I (0.28 to 6.96$)$

Marroni $1999^{22}$

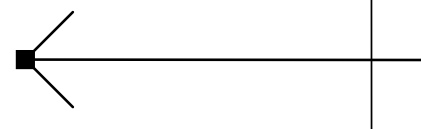

0.00 (* to I.26)

$\operatorname{RR}(95 \% \mathrm{Cl})$

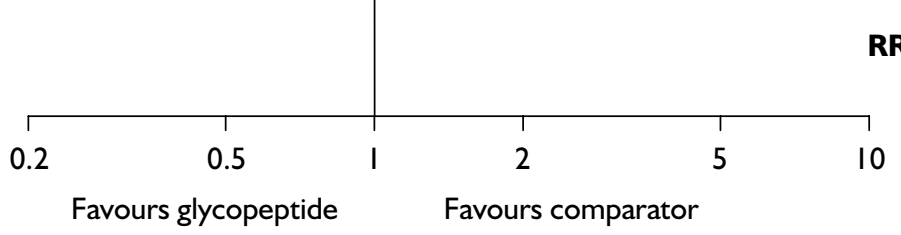

FIGURE 8 Bloodstream infections

Study

Saginur $2000^{18}$ Salminen $1999^{19}$

Maki $1992^{21}$

Kester $1999^{23}$

Marroni $1999^{22}$

Caprioli $1995^{14}$

Periti $1999^{24}$

Suter $1994^{25}$

Pons $1993^{28}$

Ratto $1990^{29}$

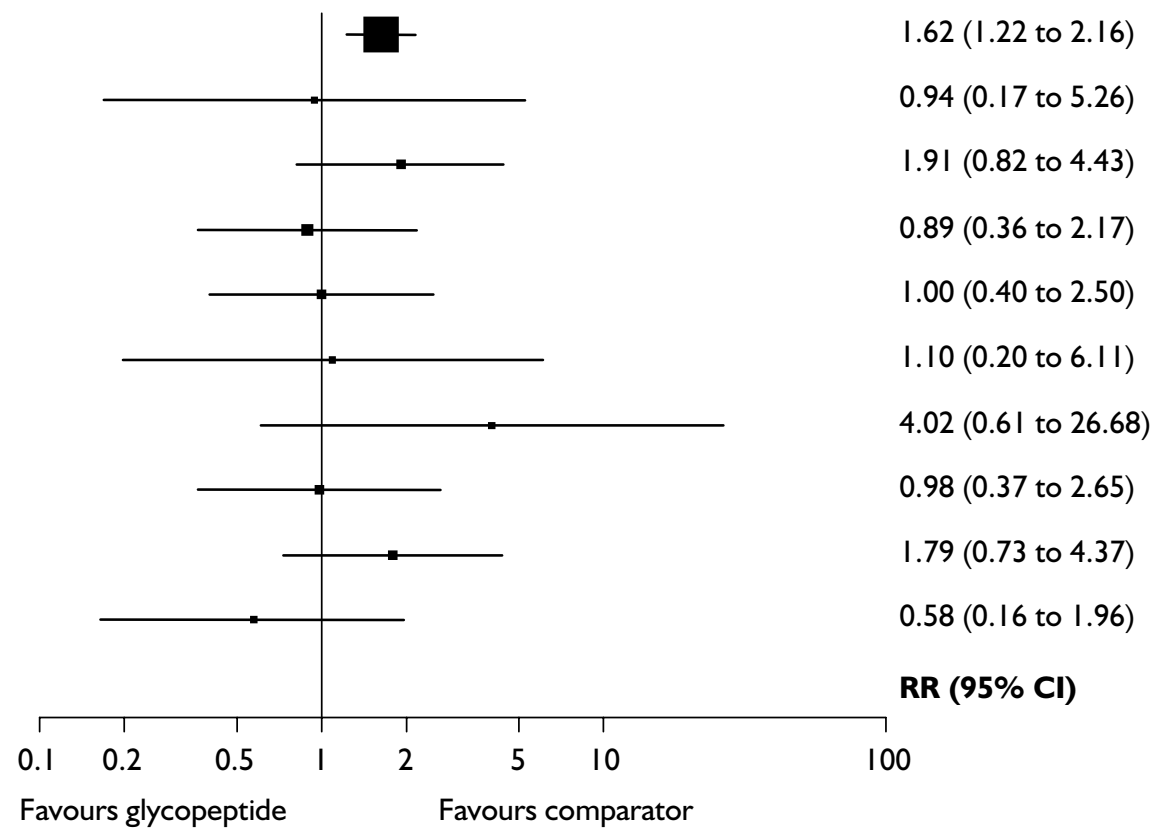




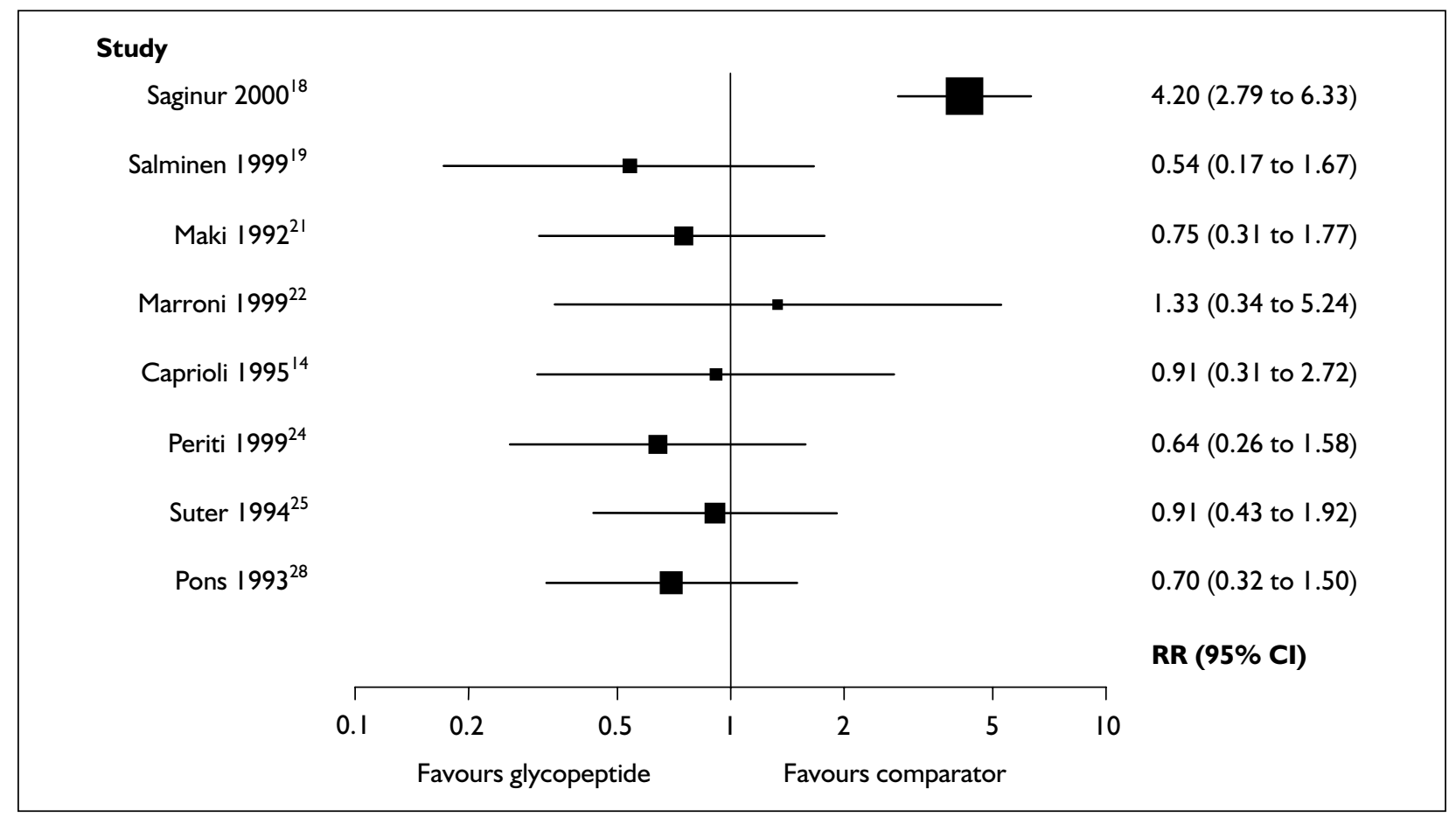

FIGURE 10 Urinary tract infections

TABLE 6 Other infections

\begin{tabular}{|c|c|c|c|c|c|}
\hline Surgery & Study & Glycopeptide & $n / N$ & Comparator & $n / N$ \\
\hline $\begin{array}{l}\text { Bloodstream } \\
\text { Cardiac }\end{array}$ & $\begin{array}{l}\text { Finkelstein, } 2002^{17} \\
\text { Saginur, } 2000^{18}\end{array}$ & $\begin{array}{l}\text { Vancomycin } \\
\text { Teicoplanin }\end{array}$ & $\begin{array}{l}20 / 452 \\
15 / 1518\end{array}$ & $\begin{array}{l}\text { Cefazolin } \\
\text { Cefazolin }\end{array}$ & $\begin{array}{l}18 / 433 \\
11 / 1509\end{array}$ \\
\hline Cardiac/vascular & Maki, $1992^{21}$ & Vancomycin & $2 / 107$ & Cefazolin & $3 / 227$ \\
\hline Vascular & Marroni, $1999^{22}$ & Teicoplanin & $0 / 119$ & Cefazolin & $3 / 119$ \\
\hline $\begin{array}{l}\text { Respiratory trac } \\
\text { Cardiac }\end{array}$ & $\begin{array}{l}\text { Saginur, } 2000^{18} \\
\text { Salminen, } 1999^{19} \text { (at } 7 \text { days) }\end{array}$ & $\begin{array}{l}\text { Teicoplanin } \\
\text { Vancomycin }\end{array}$ & $\begin{array}{c}116 / 1518 \\
2 / 103\end{array}$ & $\begin{array}{l}\text { Cefazolin } \\
\text { Ceftriaxone }\end{array}$ & $\begin{array}{c}71 / 1509 \\
2 / 97\end{array}$ \\
\hline Cardiac/vascular & Maki, $1992^{21}$ & Vancomycin & $9 / 107$ & Cefazolin & $10 / 227$ \\
\hline Vascular & $\begin{array}{l}\text { Kester, } 1999^{23} \\
\text { Marroni, } 1999^{22} \text { (pneumonia) }\end{array}$ & $\begin{array}{l}\text { Teicoplanin } \\
\text { Teicoplanin }\end{array}$ & $\begin{array}{l}8 / 136 \\
8 / 119\end{array}$ & $\begin{array}{l}\text { Cefradine } \\
\text { Cefazolin }\end{array}$ & $\begin{array}{l}9 / 136 \\
8 / 119\end{array}$ \\
\hline Orthopaedic & $\begin{array}{l}\text { Caprioli, } 1995^{14} \text { (pneumonia/pleuritis) } \\
\text { Periti, } 1999^{24} \\
\text { Suter, } 1994^{25}\end{array}$ & $\begin{array}{l}\text { Vancomycin } \\
\text { Teicoplanin } \\
\text { Teicoplanin }\end{array}$ & $\begin{array}{l}2 / 83 \\
4 / 422 \\
7 / 250\end{array}$ & $\begin{array}{l}\text { Cefamandole } \\
\text { Cefazolin } \\
\text { Cefamandole }\end{array}$ & $\begin{array}{l}2 / 91 \\
1 / 424 \\
7 / 246\end{array}$ \\
\hline Neurosurgery & Pons, $1993^{28}$ (pneumonia) & Vancomycin & $12 / 404$ & Ceftizoxime & $7 / 422$ \\
\hline Thoracic & Ratto, $1990^{29}$ & $\begin{array}{l}\text { Teicoplanin } \\
\text { (long-term) }\end{array}$ & $3 / 25$ & $\begin{array}{l}\text { Teicoplanin } \\
\text { (short-term) }\end{array}$ & $5 / 24$ \\
\hline $\begin{array}{l}\text { Urinary tract } \\
\text { Cardiac }\end{array}$ & $\begin{array}{l}\text { Saginur, } 2000^{18} \\
\text { Salminen, } 1999^{19}\end{array}$ & $\begin{array}{l}\text { Teicoplanin } \\
\text { Vancomycin }\end{array}$ & $\begin{array}{c}114 / 1518 \\
4 / 103\end{array}$ & $\begin{array}{l}\text { Cefazolin } \\
\text { Ceftriaxone }\end{array}$ & $\begin{array}{c}27 / 1509 \\
7 / 97\end{array}$ \\
\hline Cardiac/vascular & Maki, $1992^{21}$ & Vancomycin & $6 / 107$ & Cefazolin & $17 / 227$ \\
\hline Vascular & Marroni, $1999^{22}$ & Teicoplanin & $4 / 119$ & Cefazolin & $3 / 119$ \\
\hline Orthopaedic & $\begin{array}{l}\text { Caprioli, } 1995^{14} \\
\text { Periti, } 1999^{24} \text { (UTI + bacteriuria) } \\
\text { Suter, } 1994^{25}\end{array}$ & $\begin{array}{l}\text { Vancomycin } \\
\text { Teicoplanin } \\
\text { Teicoplanin }\end{array}$ & $\begin{array}{c}5 / 83 \\
7 / 422 \\
12 / 250\end{array}$ & $\begin{array}{l}\text { Cefamandole } \\
\text { Cefazolin } \\
\text { Cefamandole }\end{array}$ & $\begin{array}{l}6 / 9 \mid \\
\mid 1 / 424 \\
13 / 246\end{array}$ \\
\hline Neurosurgery & Pons, $1993^{28}$ & Vancomycin & $10 / 404$ & Ceftizoxime & $15 / 422$ \\
\hline
\end{tabular}


trial of cardiac and vascular surgery by Maki and colleagues, ${ }^{21}$ none of the remote infections were caused by MRSA and two (1.8\%) of the cefamandole patients and none of the vancomycin patients had remote infections caused by MR-CNS. None of the other trials reported the bacteriology of remote infections.

\section{Mortality}

Six trials ${ }^{17,18,20,22,25,29}$ reported deaths from any cause (Figure 11 and Table 7) and four trials ${ }^{18,20,22,23}$ reported deaths that were caused by or related to an infection (Figure 12 and Table 7). No statistically significant differences between glycopeptide and cephalosporin prophylaxis were observed in any of the trials and the numbers of infection-related deaths were generally small. In the trial of vascular surgery by Marroni and colleagues, one teicoplanin patient died from a prosthesis related infection and one cefazolin patient died from a bloodstream infection. ${ }^{22}$ In the two cardiac surgery trials, Saginur and colleagues reported that 14 $(0.9 \%)$ teicoplanin and $12(0.8 \%)$ cefazolin patients had ongoing infections at the time of death; ${ }^{18}$ and Vuorisalo and colleagues reported no infectionrelated deaths amongst patients receiving vancomycin but one cefuroxime patient died from mediastinitis caused by MR-CNS. ${ }^{20}$

\section{Length of postoperative stay}

Seven trials reported the length of postoperative hospital stay (Figure 13 and Table 8). ${ }^{17,18,20,21,23-25}$
Two trials reported the mean length of stay but not the associated standard deviation (SD) and are not included in Figure 13. The trial by Maki and colleagues was the only trial to report a statistically significant difference between the glycopeptide and cephalosporin arms. ${ }^{21}$ Patients receiving vancomycin stayed in hospital for less time than those receiving either cefazolin or cefamandole (mean difference 1.9 days, $95 \%$ CI 0.2 to 3.5 days).

\section{Rehospitalisation and reoperation}

Only one trial, of cardiac surgery by Saginur and colleagues, reported the incidence of rehospitalisation. ${ }^{18}$ A total of $320(21.1 \%)$ teicoplanin and 297 (19.7\%) cefazolin patients were rehospitalised after surgery and $52(3.4 \%)$ teicoplanin and 42 cefazolin $(2.8 \%)$ patients underwent repeat surgery. It was not reported if any of these were related to an infection.

\section{Morbidity}

There were no reports of cases of morbidity or disability related to infections.

\section{Adverse events}

Nine studies included in the effectiveness review reported on AEs possibly or probably related to antibiotic prophylaxis. ${ }^{13,18,21,23-26,28,29}$ Of these, one study compared vancomycin with teicoplanin ${ }^{13}$ and one compared long-term and short-term teicoplanin prophylaxis. ${ }^{29}$ Three studies were included in the review for data on

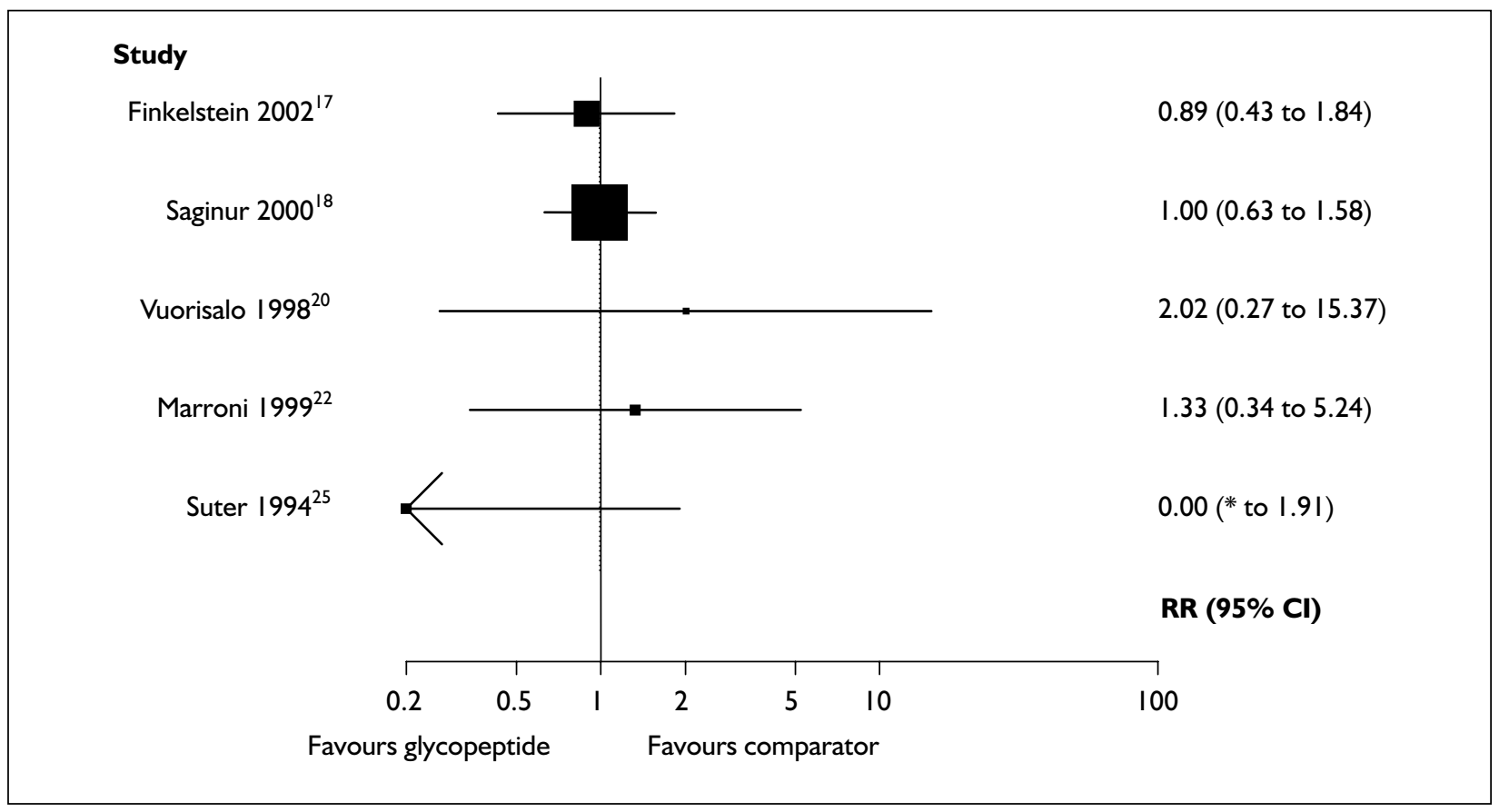


AEs only. ${ }^{15,30,31}$ One study compared vancomycin with cefamandole (both with or without aprotinin), ${ }^{30}$ although its aim was to assess the effect of these drugs on kidney function. Miro and colleagues reported adverse events from an open, non-randomised study comparing patients undergoing $\mathrm{CABG}$ or valve replacement from 1992 to 1994 who received teicoplanin prophylaxis with patients from 1990 to 1992 who received vancomycin. ${ }^{15}$ Romanelli and colleagues randomised patients undergoing CABG to vancomycin or placebo (in addition to cefazolin) to assess the haemodynamic effects of vancomycin administration. ${ }^{31}$ All AE data are summarised in Table 9.

'Red man syndrome' is an adverse reaction to vancomycin often attributed to rapid drug infusion, with symptoms including hypotension, pruritis and flushing of the face, neck and upper torso. Six studies reported the occurrence of hypotension or red man syndrome after vancomycin administration, with the numbers of patients affected ranging from 0.2 to $50 \%$. Two

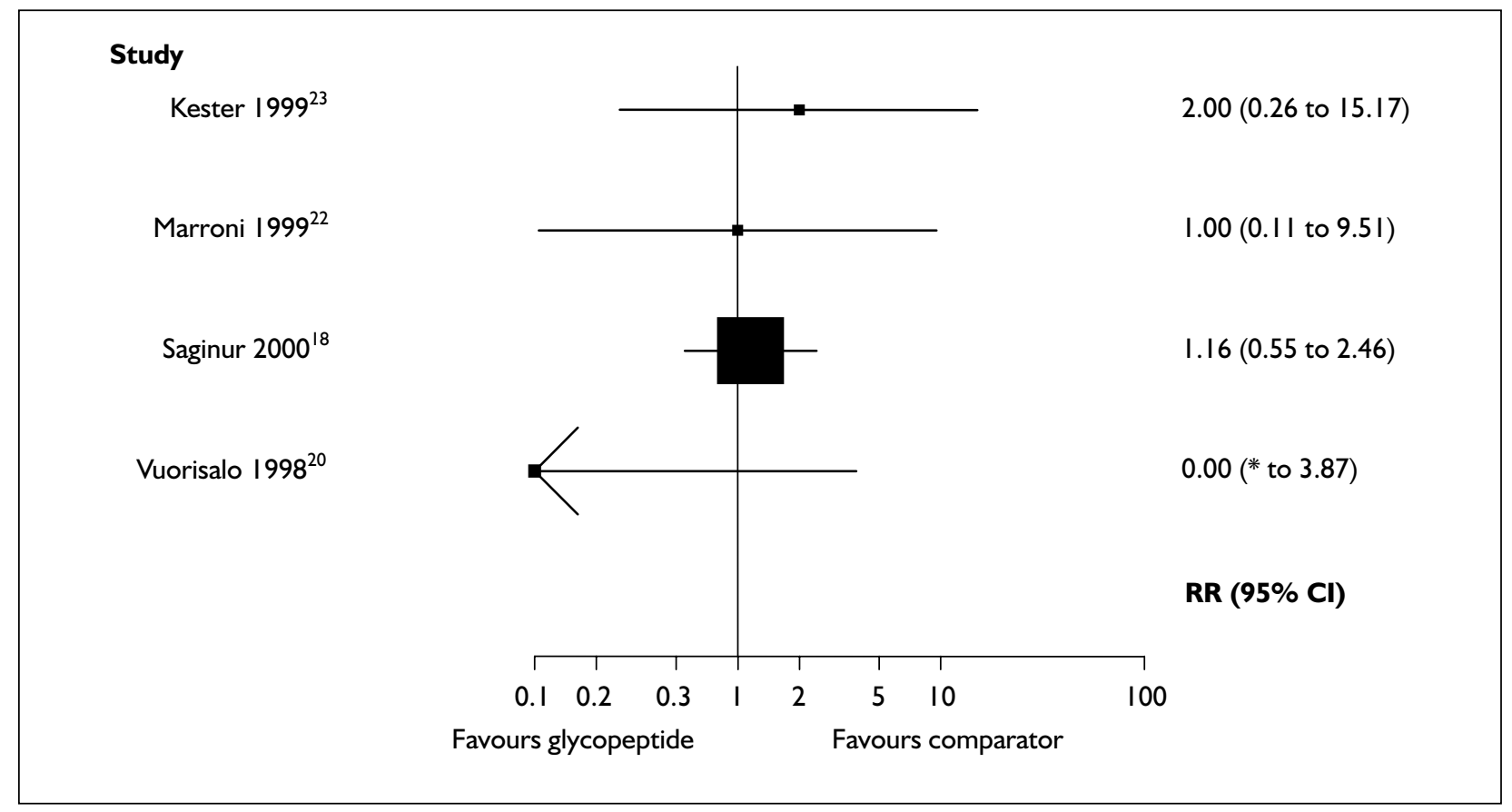

FIGURE I2 Death relating to infection

TABLE 7 Mortality

\begin{tabular}{|c|c|c|c|c|c|}
\hline Surgery & Study & Glycopeptide & $n / N$ & Comparator & $n / N$ \\
\hline \multicolumn{6}{|c|}{ Death from any cause } \\
\hline Cardiac & $\begin{array}{l}\text { Finkelstein, } 2002^{17} \\
\text { Saginur, } 2000^{18} \\
\text { Vuorisalo, } 1998^{20}\end{array}$ & $\begin{array}{l}\text { Vancomycin } \\
\text { Teicoplanin } \\
\text { Vancomycin }\end{array}$ & $\begin{array}{l}13 / 452 \\
35 / 1527 \\
2 / 440\end{array}$ & $\begin{array}{l}\text { Cefazolin } \\
\text { Cefazolin } \\
\text { Cefuroxime }\end{array}$ & $\begin{array}{c}14 / 433 \\
35 / 1520 \\
1 / 444\end{array}$ \\
\hline Vascular & Marroni, $1999^{22}$ & Teicoplanin & $4 / 119$ & Cefazolin & $3 / 119$ \\
\hline Orthopaedic & Suter, $1994^{25}$ & Teicoplanin & $0 / 260$ & Cefamandole & $2 / 260$ \\
\hline Thoracic & Ratto, $1990^{29}$ & $\begin{array}{l}\text { Teicoplanin } \\
\text { (long-term) }\end{array}$ & $1 / 25$ & $\begin{array}{l}\text { Teicoplanin } \\
\text { (short-term) }\end{array}$ & $0 / 24$ \\
\hline \multicolumn{6}{|c|}{ Death relating to infection } \\
\hline Vascular & $\begin{array}{l}\text { Kester, } 1999^{23} \\
\text { Marroni, } 1999^{22}\end{array}$ & $\begin{array}{l}\text { Teicoplanin } \\
\text { Teicoplanin }\end{array}$ & $\begin{array}{l}2 / 136 \\
1 / 119\end{array}$ & $\begin{array}{l}\text { Cefradine } \\
\text { Cefazolin }\end{array}$ & $\begin{array}{l}1 / 136 \\
1 / 119\end{array}$ \\
\hline Cardiac & $\begin{array}{l}\text { Saginur, } 2000^{18} \\
\text { Vuorisalo, } 1998^{20}\end{array}$ & $\begin{array}{l}\text { Teicoplanin } \\
\text { Vancomycin }\end{array}$ & $\begin{array}{l}14 / 1527 \\
0 / 440\end{array}$ & $\begin{array}{l}\text { Cefazolin } \\
\text { Cefuroxime }\end{array}$ & $\begin{array}{l}12 / 1520 \\
1 / 444\end{array}$ \\
\hline
\end{tabular}




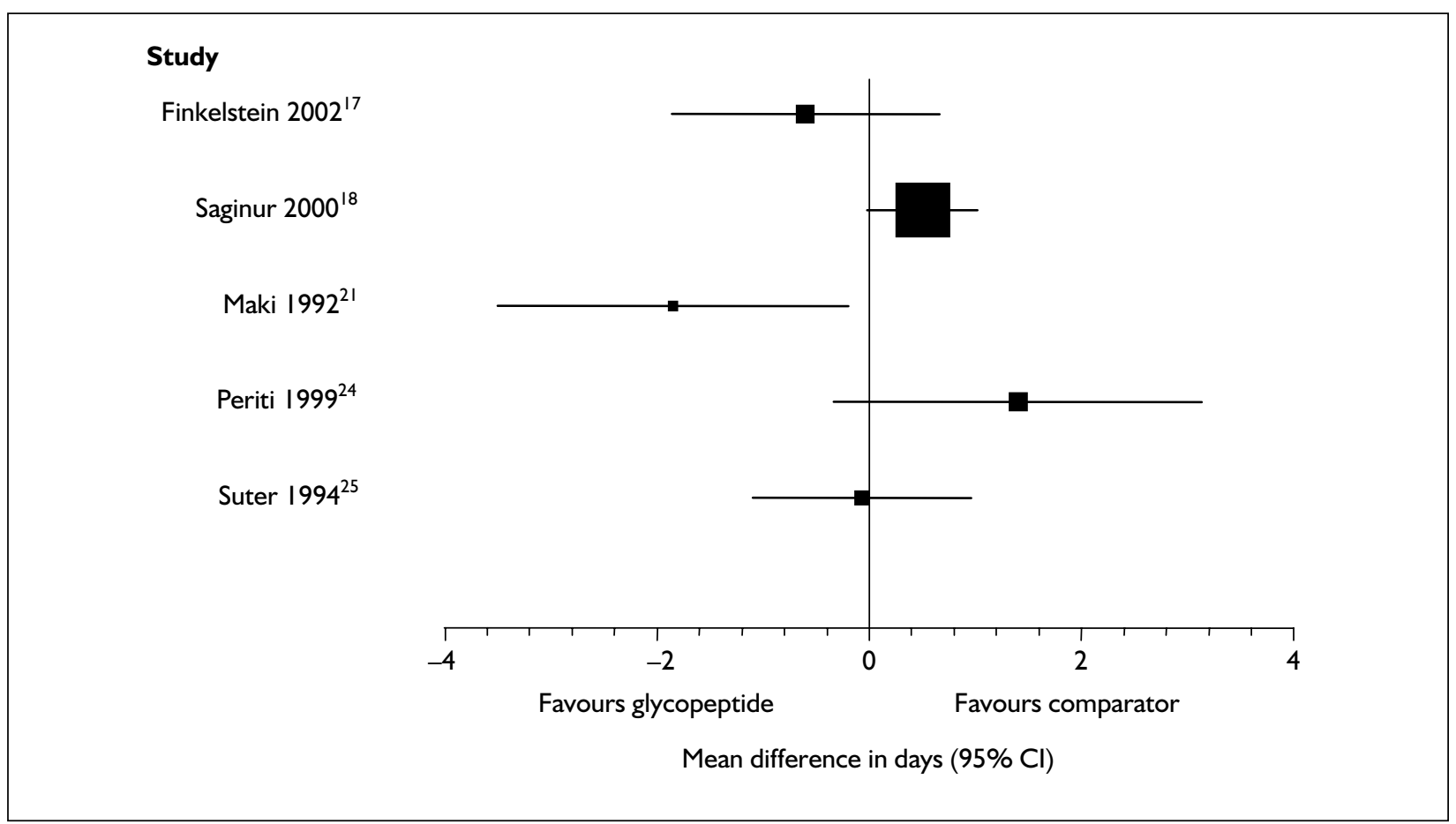

FIGURE 13 Length of postoperative stay

TABLE 8 Length of postoperative stay (days)

\begin{tabular}{|c|c|c|c|c|c|c|c|}
\hline Surgery & Study & Glycopeptide & Mean & SD & Comparator & Mean & SD \\
\hline Cardiac & $\begin{array}{l}\text { Finkelstein, } 2002^{17} \\
\text { Saginur, } 2000^{18} \\
\text { Vuorisalo, } 1998^{20}\end{array}$ & $\begin{array}{l}\text { Vancomycin } \\
\text { Teicoplanin } \\
\text { Vancomycin }\end{array}$ & $\begin{array}{l}8.7 \\
10 \\
11\end{array}$ & $\begin{array}{l}8 \\
7.9 \\
N R\end{array}$ & $\begin{array}{l}\text { Cefazolin } \\
\text { Cefazolin } \\
\text { Cefuroxime }\end{array}$ & $\begin{array}{r}9.3 \\
9.5 \\
11\end{array}$ & $\begin{array}{l}11 \\
6.7 \\
N R\end{array}$ \\
\hline Cardiac/vascular & Maki, $1992^{21}$ & Vancomycin & 10.1 & 6.1 & $\begin{array}{l}\text { Cefazolin or } \\
\text { cefamandole }\end{array}$ & 12 & 7.63 \\
\hline Vascular & Kester, $1999^{23}$ & Teicoplanin & 15.4 & $N R$ & Cefradine & 20.8 & NR \\
\hline Orthopaedic & $\begin{array}{l}\text { Periti, } 1999^{24} \\
\text { Suter, } 1994^{25}\end{array}$ & $\begin{array}{l}\text { Teicoplanin } \\
\text { Teicoplanin }\end{array}$ & $\begin{array}{l}24.4 \\
17.04\end{array}$ & $\begin{array}{r}13.7 \\
6.55\end{array}$ & $\begin{array}{l}\text { Cefazolin } \\
\text { Cefamandole }\end{array}$ & $\begin{array}{l}23 \\
17.11\end{array}$ & $\begin{array}{l}12 \\
5.02\end{array}$ \\
\hline
\end{tabular}

studies reported red man syndrome, which occurred in $1.5 \%(6 / 404)$ of vancomycin patients compared with no ceftizoxime patients in one study, ${ }^{28}$ and $5 \%(37 / 736)$ of vancomycin patients compared with $1.1 \%(7 / 656)$ of teicoplanin patients in the other study. ${ }^{15}$

Four studies reported on hypotension with serious hypotension in one study (defined by the study as a reduction in systolic arterial pressure greater than $30 \%$ ) occurring in one patient $(0.4 \%)$ in each of the vancomycin and teicoplanin groups. ${ }^{13}$ In a second study, hypotension during drug administration (pre- or postoperative) occurred in
$(5 / 227)$ of control patients (receiving cefamandole or cefazolin); this resolved in most patients when infusion was stopped and resumed at a lower rate but three vancomycin and two control patients were removed from the trial. ${ }^{21}$ Hypotension requiring a norepinephrine infusion occurred in $5 \%(37 / 736)$ of vancomycin plus cefazolin patients compared with $14.3 \%$ (4/28) of cefazolin patients in a third study, although the aim of this study was to assess the haemodynamic effects of drug infusion, not its prophylactic effectiveness. ${ }^{31}$ One teicoplanin study also reported hypotension, which occurred in three teicoplanin patients $(0.2 \%)$ compared with five $(0.3 \%)$ cefazolin patients. $^{18}$ 
TABLE 9 Adverse events possibly or probably related to antibiotic prophylaxis

\begin{tabular}{|c|c|c|c|c|c|}
\hline Study & Adverse event & Glycopeptide & $n / N$ & Comparator & $n / N$ \\
\hline \multicolumn{6}{|c|}{ Studies included for effectiveness } \\
\hline \multirow[t]{3}{*}{ Codina, $2000^{13}$} & $A E$ after first dose & Vancomycin & $51 / 250$ & Teicoplanin & $4 / 250$ \\
\hline & $\mathrm{AE}$ after second dose & Vancomycin & $6 / 118$ & Teicoplanin & $1 / 115$ \\
\hline & Hypotension (considered serious) & Vancomycin & $\mathrm{I} / 250$ & Teicoplanin & $1 / 250$ \\
\hline Kester, $1999^{23}$ & $\begin{array}{l}\text { Event possibly or probably related to } \\
\text { drug }\end{array}$ & Teicoplanin & $19 / 136$ & Cefradine & $15 / 136$ \\
\hline \multirow[t]{4}{*}{ Maki, $1992^{21}$} & $\begin{array}{l}\text { C. difficile antibiotic-associated colitis } \\
\text { (mild) }\end{array}$ & Vancomycin & $0 / 107$ & Cefamandole/cefazolin & $2 / 227$ \\
\hline & Maculopapular rash (mild) & Vancomycin & $1 / 107$ & Cefamandole/cefazolin & $3 / 227$ \\
\hline & $\begin{array}{l}\text { Hypotension during administration } \\
\text { (pre- or post-operative)) }\end{array}$ & Vancomycin & $8 / 107$ & Cefamandole/cefazolin & $5 / 227$ \\
\hline & $\begin{array}{l}\text { Patient removed from study due to } \\
\text { hypotension }\end{array}$ & Vancomycin & $3 / 107$ & Cefamandole/cefazolin & $2 / 227$ \\
\hline \multirow[t]{2}{*}{ Mollan, $1992^{26}$} & Vomiting $^{a}$ & Teicoplanin & $1 / 394$ & Cefamandole & $\mathrm{I} / 407$ \\
\hline & Pruritis/flushing & Teicoplanin & $0 / 394$ & Cefamandole & $\mathrm{l} / 407$ \\
\hline \multirow[t]{2}{*}{ Periti, $1999^{24}$} & Erythema & Teicoplanin & $0 / 394$ & Cefazolin & $1 / 424$ \\
\hline & Rash & Teicoplanin & $0 / 394$ & Cefazolin & $1 / 424$ \\
\hline Pons, $1993^{28}$ & $\begin{array}{l}\text { Red man syndrome (hypotension } \\
\text { and/or flushing) }\end{array}$ & Vancomycin & $6 / 404$ & Ceftizoxime & $0 / 422$ \\
\hline Ratto, $1990^{29}$ & Postoperative diarrhoea & $\begin{array}{l}\text { Teicoplanin } \\
\text { (long-term) }\end{array}$ & $3 / 25$ & $\begin{array}{l}\text { Teicoplanin } \\
\text { (short-term) }\end{array}$ & $1 / 24$ \\
\hline \multirow[t]{5}{*}{ Saginur, $2000^{18}$} & Anaphylactic shock & Teicoplanin & $1 / 1518$ & Cefazolin & $1 / 1509$ \\
\hline & Hypotension & Teicoplanin & $3 / 1518$ & Cefazolin & $5 / 1509$ \\
\hline & Nausea & Teicoplanin & $30 / 1518$ & Cefazolin & $22 / 1509$ \\
\hline & Rash & Teicoplanin & $4 / 1518$ & Cefazolin & $3 / 1509$ \\
\hline & Vomiting & Teicoplanin & $12 / 1518$ & Cefazolin & $11 / 1509$ \\
\hline Suter, $1994^{25}$ & Allergic reaction after first injection & Teicoplanin & $1 / 260$ & Cefamandole & $2 / 260$ \\
\hline \multicolumn{6}{|c|}{ Studies included for adverse events only } \\
\hline \multirow[t]{2}{*}{ Mercieri, $1999^{30}$} & Acute renal failure (no dialysis required) & Vancomycin & $0 / 21$ & Cefamandole & $1 / 18$ \\
\hline & Acute renal failure (no dialysis required) & $\begin{array}{l}\text { Vancomycin } \\
\text { (plus aprotonin) }\end{array}$ & $1 / 23$ & $\begin{array}{l}\text { Cefamandole } \\
\text { (plus aprotonin) }\end{array}$ & $0 / 22$ \\
\hline Miro, $1996^{15}$ & Severe hypotension/red man syndrome & Vancomycin & $37 / 736$ & Teicoplanin & $7 / 656$ \\
\hline Romanelli, $1993^{31}$ & $\begin{array}{l}\text { Hypotension requiring a } \\
\text { norepinephrine infusion }\end{array}$ & $\begin{array}{l}\text { Vancomycin } \\
\text { (plus cefazolin) }\end{array}$ & $15 / 30$ & Cefazolin & $4 / 28$ \\
\hline
\end{tabular}

Other AEs reported were generally mild and included nausea, vomiting and rash. In general, rates of these were low and similar between glycopeptide and comparator groups. One study that reported a higher rate of events in the vancomycin arm (20.4\% of patients after the first dose) found a higher incidence of AEs after the both the first and second doses compared with teicoplanin $(1.6 \%)$, although these were not considered to be serious and were mainly erythema and pruritis. ${ }^{13}$

\section{Discussion}

Only one trial of cardiac and vascular surgery found a statistically significant reduction in the numbers of SSIs occurring within 30 days of 
surgery for patients receiving vancomycin compared with cefazolin or cefamandole. ${ }^{21}$ Most of the trials did not report MRSA infections: two trials provided results for the numbers of infections caused by MRSA; three trials reported that no MRSA infections occurred; and one trial reported the number of MRSA infections overall but not separately for the glycopeptide and comparator groups. Both trials that provided MRSA results reported fewer MRSA infections in the glycopeptide arm but the results were not statistically significant. One of these was the only trial to report both MRSA prevalence and MRSA infections and was conducted in a cardiac unit with a high MRSA prevalence. ${ }^{17}$ Only one trial reported the occurrence of infections caused by MRSE. There was a limited amount of evidence that glycopeptide prophylaxis might be more effective in preventing infections caused by methicillin-resistant bacteria (including MRSA, MR-CNS and MRSE) with fewer infections reported in the glycopeptide arms of four trials, although this was only statistically significant in one trial. $^{21}$

With the exception of one trial, ${ }^{17}$ the prevention of MRSA infections was not the primary objective and most did not report the baseline prevalence of MRSA. This confirms the findings of the review by Bolon and colleagues which found no evidence to support a switch from beta-lactams to glycopeptide prophylaxis in cardiac surgery. ${ }^{32}$ Only two trials reported testing for glycopeptide-resistance and both found no resistance to teicoplanin.

Many of the trials included in the effectiveness review had weaknesses in design and/or reporting. Very few reported an a priori sample size calculation and so were likely to have been underpowered for detecting a difference in SSI rates. The largest and best conducted trial was in cardiac surgery, ${ }^{18}$ but this did not provide any comparative results for MRSA infections or report prevalence (other than saying it was low). Poor reporting may reflect the fact that many of these trials were published before the CONSORT standards for reporting of RCTs were widely adopted. ${ }^{33}$ Follow-up beyond 12 months was rarely reported, which may be an issue for orthopaedic surgery, where deep infections occurring some time after surgery are a major concern.

Most trials gave antibiotics at induction of anaesthesia, although some were earlier. Typical doses were $1 \mathrm{~g}$ for vancomycin, $400 \mathrm{mg}$ for teicoplanin and 1 or $2 \mathrm{~g}$ for cephalosporin comparators. Overall, there was not enough evidence to judge whether different doses and/or durations affected the risk of developing an infection. None of the trials evaluated the effect of varying dose or time of administration. There was also limited information on the effects of single compared with combined antibiotic regimens (no direct comparisons). Two orthopaedic trials assessed a glycopeptide plus gentamicin (alone or in bone cement), a neurosurgery trial assessed vancomycin plus gentamicin and one cardiac trial assessed vancomycin plus netilmicin.

The most common comparators (four trials each) were cefazolin and cefamandole. Third-generation cephalosporins were used as comparators in only two trials. Only one trial compared a glycopeptide with two different cephalosporins and the results for SSIs were similar for each cephalosporin. ${ }^{21}$

Most trials in the effectiveness review reported AEs and three additional studies were included for AEs only. The AE rates were generally low and most were mild such as nausea and vomiting. More serious events such as red man syndrome or serious hypotension were more common with vancomycin in four of the five studies that reported their occurrence. AEs were more frequent with vancomycin than with teicoplanin in direct comparisons. ${ }^{13,15}$ Any increase in infection prophylaxis from a switch to vancomycin from cephalosporins needs to be balanced against an increased risk of AEs.

\section{Summary}

The systematic review of effectiveness found that there was insufficient evidence available to suggest a threshold level of MRSA prevalence at which it would be appropriate to switch from nonglycopeptide to glycopeptide antibiotics for surgical infection prophylaxis. There was also a lack of evidence from RCTs to conclude that glycopeptide regimens are more effective than non-glycopeptides in preventing SSIs. Most studies did not test for drug-resistant bacteria, so the effectiveness review is unable to provide any information about the risk of increasing bacterial resistance to glycopeptides. 


\section{Chapter 4}

\section{Systematic review of cost-effectiveness}

\section{Methods}

The aim of the systematic review of published economic evaluations was to examine the costeffectiveness of glycopeptide antibiotics compared with appropriate comparators.

\section{Search strategy}

The literature searches for this review were undertaken in the same databases used for the effectiveness review (see Chapter 3).

Additional searches of the following economics databases were also undertaken (the search strategy is presented in Appendix 1):

- NHS EED

- HEED

- IDEAS.

\section{Inclusion criteria}

Studies that were full economic evaluations were considered, that is: (1) cost-effectiveness evaluations, including cost-minimisation and cost-consequence analyses; (2) cost-utility analyses; and (3) cost-benefit analyses. ${ }^{34}$ At least one of the interventions had to be a glycopeptide used as antibiotic prophylaxis for surgery.

\section{Data extraction and quality assessment}

Data were extracted on key components of standard economic evaluations, including data on the comparators, study population, main analytic approaches (e.g. patient-level analysis/decision analytic modelling), primary outcome specified for the economic analysis, details of adjustment for quality of life, direct costs (medical and nonmedical) and productivity costs, estimates of incremental cost-effectiveness and approaches to quantifying decision uncertainty (e.g. deterministic/probabilistic sensitivity analysis). Additional data were captured on whether or not screening and/or diagnostic tests for bacterial colonisation were undertaken, the type of surgery, the surgical site, the surgical environment, the method of administration of the prophylaxis, the use of other interventions to prevent/reduce infection rate and methods used to assess antibiotic resistance).
The quality of the cost-effectiveness studies was assessed according to a list updated from that developed by Drummond and colleagues. ${ }^{34}$ The list of items indicated components of 'good quality' in a standardised manner (see Appendix 2).

\section{Results}

\section{Nature of the evidence}

Fourteen potential studies were identified from the search of the NHS EED database. No additional studies were identified from other economic databases or the searches for the clinical effectiveness review. Once the inclusion/exclusion criteria were applied, five relevant costeffectiveness studies of glycopeptide antibiotic prophylaxis were included. ${ }^{13,22,35-37}$ All studies focused on clean surgery with the exception of that by Phillips and colleagues. ${ }^{35}$ Surgical sites covered were cardiac, ${ }^{13,36}$ cardiothoracic, ${ }^{37}$ prosthetic vascular ${ }^{22}$ and cardiovascular. ${ }^{35}$ The full data extraction tables are presented in Appendix 5 and the quality assessment in Appendix 6.

Glycopeptides (teicoplanin or vancomycin) were compared with each other or with cefazolin. No studies compared all glycopeptide and nonglycopeptide antibiotic prophylaxis which are relevant to this review. The following antibiotics were compared: vancomycin versus teicoplanin, ${ }^{13}$ teicoplanin versus cefazolin, ${ }^{22}$ vancomycin versus cefazolin $^{35,37}$ and vancomycin and rifampicin versus cefazolin. ${ }^{36}$

Glycopeptides were administered at induction of anaesthesia as single doses [coronary artery bypass graft (CABG) $\operatorname{arm}^{13,22}$ ] or perioperatively as multiple doses [in the valve replacement (VR) $\left.\operatorname{arm}^{13,35-37}\right]$. The duration of time over which multiple doses were administered was not always stated. In two studies it was stated that the glycopeptides were administered intravenously ${ }^{13,22}$ and in the other studies no mention was made about the administration of the antibiotics.

Two studies were based on prospective doubleblind RCTs, ${ }^{13,22}$ two studies included a 
hypothetical cohort of patients using effectiveness data obtained from the published literature ${ }^{35,37}$ and one economic evaluation was based on data from a single hospital where routine use of antibiotic prophylaxis changed from cephazolin to vancomycin and rifampicin. ${ }^{36}$ Three studies undertook some form of decision analysis to synthesise data. ${ }^{13,35,37}$ One model was informed by a single $\mathrm{RCT}^{13}$ and the other models were informed by reviews of the literature. Little detail was provided about the sources searched or the designs of the studies from which data were obtained to inform the models. The sample size of patients varied from 119 patients in one arm of a trial in one study ${ }^{22}$ to 599 in one arm of a trial in another study. ${ }^{36}$ Two studies were based on decision analytic models and included a hypothetical cohort of patients. ${ }^{35,37}$

The length of patient follow-up varied considerably across studies. In one study, patients were followed up until they were discharged from hospital. ${ }^{13}$ In another study, data relating to patients 3 months post-surgery were provided for the base case and up to 5 years for the reference case. ${ }^{37}$ Marroni and colleagues included patients who were followed up for an average of 2 years. ${ }^{22}$ For the remaining two studies, there was no information on the length of patient follow-up. ${ }^{35,36}$

None of the studies were conducted in the UK. The studies were conducted in Spain, ${ }^{13}$ Italy, ${ }^{22}$ Canada, ${ }^{35}$ Australia ${ }^{36}$ and the USA. ${ }^{37}$ There may be resource implications in terms of transferring findings to the UK setting. With the exception of Zanetti and colleagues, ${ }^{37}$ all studies were undertaken from the secondary care perspective. Costs were reported in Spanish pesetas, ${ }^{13}$ US dollars $22,35,37$ and Australian dollars. ${ }^{36}$

Codina and colleagues ${ }^{13}$ undertook a costminimisation analysis comparing vancomycin with teicoplanin as antibiotic prophylaxis in elective cardiac surgery. Results were analysed by surgery type, either by CABG or VR. One group of patients were administered $1 \mathrm{~g}$ of vancomycin intravenously at induction of anaesthesia. They also received netilmicin $150 \mathrm{mg}$ and a teicoplanin placebo. Patients undergoing VR received a second dose of vancomycin at the end of extracorporeal circulation. The other group of patients were administered $400 \mathrm{mg}$ of teicoplanin intravenously at induction of anaesthesia. They also received netilmicin $150 \mathrm{mg}$ and a vancomycin placebo. Patients undergoing VR received a second dose (200 $\mathrm{mg}$ of teicoplanin) at the end of extracorporeal circulation.
The analysis was based on a single-centre, doubleblind, parallel-group RCT conducted in Spain and was undertaken from the secondary care perspective. There were 250 patients in each group, 233 undergoing VR surgery and 267 undergoing CABG. Patients were followed up until they were discharged from hospital.

The primary health outcomes that were measured included the rate of adverse drug reactions and the postoperative infection rates. No statistically significant differences were found in adverse drug reactions or postoperative infection rates for patients in the vancomycin group compared with the teicoplanin group. On this basis, the authors assumed that the health outcomes were equivalent across the interventions and therefore they based their evaluation on the difference in costs between the interventions.

Costs comprised the cost of the drug, the intravenous mix and the administration costs, together with personnel, capital and overhead costs, and this was consistent with the hospital perspective adopted by the study. Resource use was collected prospectively and was reported separately from costs, in appropriate physical units, helping to make the analysis transparent and potentially aiding study generalisability.

A decision tree was used to evaluate two scenarios: antibiotic administration in (1) the surgical room theatre and (2) the medical ward. For the CABG patients, when the antibiotics were administered in the surgical room, the cost was 12,005 pesetas (pts) per patient (1998 prices) for those who received vancomycin and 8265 pts for those who received teicoplanin. In contrast, when the antibiotics were administered in the medical room, the cost was 2809 pts for those who received vancomycin and 6740 pts for those who received teicoplanin. For the valve replacement patients, when the antibiotics were administered in the surgical room, the cost was 14,528 pts for those who received vancomycin and 11,661 pts for those who received teicoplanin. If the antibiotics were administered in the medical ward, the cost was 10,140 pts for those who received vancomycin and 5308 pts for teicoplanin.

The authors concluded that the cost of antibiotic prophylaxis among cardiac surgery patients depends heavily on the setting and the circumstances of the drug administration. Based on the cost-minimisation analysis, vancomycin was the least costly option when administered within the medical ward, whereas teicoplanin was the 
most cost-effective option if the drug was administered within the surgical area. The authors note that if vancomycin is used in the medical ward, it is important that the right plasmatic drug levels of the antibiotic are administered at the beginning of the surgical procedure.

The authors justified their decision to undertake a cost-minimisation analysis due to the number of adverse drug events being equivalent across the treatments. The analysis can be considered as a partial economic evaluation analysis, since the authors did not make full use of all the available outcomes data, some of which did show across comparator differences. Costs were treated deterministically; however, patient-level data did allow for stochastic analysis in which it would have been possible to quantify the uncertainty associated with the cost-effectiveness estimates. No data were reported on MRSA. The impact of antibiotic resistance on long-term effectiveness of antibiotic prophylaxis was not considered within the analysis.

Marroni and colleagues ${ }^{22}$ undertook a costminimisation analysis comparing teicoplanin with cefazolin as antibiotic prophylaxis in prosthetic vascular surgery. Adults undergoing elective abdominal aortic and lower-extremity peripheral vascular surgery were included in the study. A single intravenous 400-mg dose of teicoplanin was compared with a 2-g dose of cefazolin at the induction of anaesthesia. The analysis was based on a single-centre, double-blind RCT conducted in Italy and was undertaken from the secondary care perspective. There were 119 patients in each group and the aim was to follow up patients for 1 year post-hospital discharge. In practice, the mean length of follow-up was 24 months.

The primary health outcomes that were measured included the rate of prosthetic and wound infections. Postoperative infections were defined using the CDC criteria. Secondary health outcomes included mortality and side-effects. Antibiotic-related nephrotoxicity was considered but ototoxicity was not considered.

In total, SSIs were reported for seven (5.9\%) patients in the teicoplanin group, two $(1.7 \%)$ of which were in grafts and five $(4.2 \%)$ of which were in wounds, and two $(1.7 \%)$ patients in the cefazolin group. One patient in the teicoplanin group developed an anastomotic aneurysm and MSSA was isolated from the aneurysm: the patient was cured and had no sign of infection 24 months later.
Early superficial wound infections, diagnosed a mean of 9 days after surgery, were reported in five $(4.2 \%)$ patients in the teicoplanin group and two $(1.7 \%)$ in the cefazolin group. Of the five infections in the teicoplanin group, two were microbiologically tested as CNS plus Proteus mirabilis and Enterobacter cloacae. Of the two infections in the cefazolin group, one was identified as MSSA plus Proteus mirabilis.

Other postoperative infections reported in patients' postoperative stay included 12 in the teicoplanin group, of which eight $(7 \%)$ patients had pneumonia and four $(3 \%)$ had urinary tract infections, and 14 patients in the cefazolin group, with eight $(7 \%)$ pneumonia infections, three $(2.5 \%)$ urinary tract infections and three $(2.5 \%)$ bloodstream infections.

Four $(3.4 \%)$ deaths were reported in the teicoplanin group and three $(2.5 \%)$ in the cefazolin group. One death in each group was related to infection.

No side-effects were reported that related to antibiotic prophylaxis.

The costs that were measured included the cost of the antibiotics and the daily cost of the hospital stay. The total cost of care for the teicoplanin group was US $\$ 571,572$ and for the cefazolin group it was US\$519,062, giving a difference in costs of US $\$ 52,510$ (price year not stated). Cost savings using cefazolin were related to the lower cost of the drug and the shorter duration of the hospital stay.

The authors justified their decision not to compare costs with effects because there were no statistically significant differences in effects measured between the two groups. Sensitivity analysis was not undertaken. The analysis was deterministic. Unit cost information was not provided and this undermines the transparency of the results. The source of the price/cost data is not clear. No data were reported on MRSA. The impact of antibiotic resistance on long-term effectiveness of antibiotic prophylaxis was not considered.

Phillips and colleagues ${ }^{35}$ undertook a costeffectiveness analysis comparing six strategies for antimicrobial prophylaxis in cardiovascular surgery patients who were labelled penicillin allergic. The six strategies were:

1. Vancomycin given to all patients labelled penicillin allergic. 
2. Cefazolin given to all patients labelled penicillin allergic.

3. Obtain a history from all patients who were labelled penicillin allergic and then give (a) vancomycin to all patients with a history suggesting an immunoglobulin E (IgE)-mediated reaction to penicillin and (b) cefazolin to patients without a history of IgE-mediated reaction.

4. Administer penicillin tests to patients with a history suggesting an IgE-mediated reaction to penicillin, give vancomycin to patients with a positive skin test and cefazolin to all others.

5. Administer penicillin tests to all patients labelled penicillin allergic and then give vancomycin to patients with a positive skin test and cefazolin to all patients with a negative skin test, regardless of history.

6. Administer penicillin tests to patients with all patients labelled penicillin allergic and then give vancomycin to patients with either a positive skin test or a history suggesting an IgE-mediated reaction to penicillin and give cefazolin to all others.

The analysis was based on a decision tree, informed by data in the published literature. The analysis was undertaken in Canada, from the secondary care perspective. The study population comprised a hypothetical cohort of cardiovascular surgery patients who were labelled penicillin allergic. It is not clear whether the surgery was elective. The length of follow-up was not stated but this was likely to be the length of the postoperative stay in hospital.

Two primary health outcomes were measured, that is, the rate of serious non-life-threatening reactions and the rate of potentially lifethreatening anaphylactic episodes. The rate of serious non-life threatening reactions was 0.03 with strategies (1), (2) and (3), 0.02 with strategy (4) and 0.0175 with strategies (5) and (6). The rate of potentially life-threatening anaphylactic episodes was 0.0004 with strategy (1), 0.0003 with strategy (2), 0.0002 with strategy (3), 0.00027 with strategy (4), 0.00021 with strategy (5) and 0.000134 with strategy (6).

Costs included the cost of the antibiotics, the penicillin and cephalosporin skin tests and the treatment of serious adverse reaction to vancomycin and cefazolin. The authors did not provide details on resource use. Data on unit costs were reported; however, the price year was not provided.

An incremental cost-effectiveness analysis was calculated to combine the costs and benefits of the six strategies, relative to strategy (2). Based on the rate of serious non-life-threatening reactions, strategy (2) dominated strategies (1) and (3). The incremental cost per serious non-life-threatening reaction avoided with strategy (4) was US $\$ 5426$ (price year not stated). For strategy (5), the incremental cost-effectiveness ratio (ICER) was US $\$ 10,024$ and with strategy (6) it was US\$10,906. Based on the rate of potentially life-threatening anaphylactic shock, the incremental cost per reaction avoided was US $\$ 166,667$ with strategy (3), US $\$ 159,204$ with strategy (1), US $\$ 428,571$ with strategy (4), US\$692,308 with strategy (5) and US $\$ 544,776$ with strategy (6) when compared with strategy (2). The authors concluded that selective use of vancomycin is more cost-effective than indiscriminate use of vancomycin in cardiovascular surgery patients who are labelled penicillin allergic.

Univariate sensitivity analyses were conducted to address the issue of uncertainty in the parameter estimates included in the model. All estimates were varied and, for the probability values, ranges were taken from the published literature. No justification was provided for the variation in costs. The results of the sensitivity analyses suggested that the rate of serious non-life threatening reactions had the most important impact on the ICER. It was found that unrealistic changes had to be made to parameter estimates for strategy (1) to become the most cost-effective strategy.

It is not clear how the authors obtained the estimates to include in their model or how they combined any data that they did obtain from the literature. The analysis was deterministic. No data were reported on MRSA. The impact of antibiotic resistance on long-term effectiveness of antibiotic prophylaxis was not considered.

Spelman and colleagues ${ }^{36}$ undertook a cost-consequence analysis, comparing cefazolin with vancomycin and rifampacin in antibiotic prophylaxis for cardiac surgery. It is not clear whether the surgery was elective. Intravenous cefazolin was administered in four 1 -g doses, that is, $1 \mathrm{~g}$ preoperatively and $3 \mathrm{~g}$ postoperatively. A 1 -g amount of intravenous vancomycin was administered and $600 \mathrm{mg}$ of oral rifampacin preoperatively, with a second dose of vancomycin 12 hours postoperatively. Patients were given oral rifampicin in the ward before being transferred to the operating room where, on arrival, they were given the vancomycin. The vancomycin was infused over about 1 hour. There were 599 
patients in the cefazolin group and 515 patients in the vancomycin and rifampicin group.

The data were obtained from a single hospital before and after a change in the routine use of antibiotic prophylaxis in surgery from cefazolin to vancomycin and rifampicin. The analysis was undertaken in Australia, from the secondary care perspective. The study population comprised those undergoing CABG. The length of follow-up per patient was not stated; the total length of data collection was 12 months for each type of drug regimen.

The health outcomes measured were the rate of deep sternal wound infection and the overall rate of infections. Following the switch from cefazolin, the number of deep infections fell from 28 (4.7 infections per 100 procedures, 95\% CI 3.1 to 6.7), of which 25 were deep sternal infections (4.2 per 100 procedures) to three infections $(0.6$ per 100 procedures, $95 \%$ CI 0.1 to $1.7 ; p<0.001)$. For deep sternal wound infection, the absolute risk reduction was 0.36 , the RR reduction was $86 \%$ and the number of patients needed to treat to prevent one infection was 28 .

Following the switch from cefazolin, the number of infections fell from 63 (10.5 infections per 100 procedures) to 25 (4.9 infections per 100 procedures) (95\% CI 3.2 to $7.1 ; p<0.001)$. The absolute risk reduction following use of vancomycin and rifampicin was 0.056 , the RR reduction was $55.3 \%$ and the number of patients needed to treat to prevent one infection with the new regimen was 18 .

There was a reduction in the bacteriology of SSI over the course of the study with MRSA accounting for $67 \%$ of infections when cefazolin was used and not being present when using vancomycin and rifampicin. MSSA was detected in five infected patients in the cefazolin group and in two infected patients in the vancomycin and rifampicin group. Ten skin or enteric flora were reported in each of the infected groups. There was no growth/specimen in three infected patients in the cefazolin group and four infected patients in the vancomycin and rifampicin group. There were three Enterobacteriacae infections in the cefazolin group and seven in the vancomycin and rifampicin group. Additionally, there were two other infections in the vancomycin and rifampicin group. It should be noted that no regular VRE screening was performed but there were three cases of VRE bacteraemia in each 12-month period.
Costs were based on resources used in hospital and were taken from a different patient sample compared with that used in the effectiveness study. Limited details of the cost analysis were presented. The mean length of postoperative stay related to cefazolin was 9.9 days compared with 10.2 days in the vancomycin and rifampicin group. The additional cost per deep sternal wound infection was $A \$ 31,597$ (no price date). The excess total cost of infections was $\mathrm{A} \$ 789,925$ for the cefazolin group. The gross cost savings by using vancomycin and rifampicin were $A \$ 600,343$ and the cost of this was $A \$ 23,688$, giving a net saving from using this strategy of $\mathrm{A} \$ 576,655$.

Costs and benefits were not combined. The authors concluded that cost savings were obtained and there was a statistically significant decrease in the total rates of SSI and deep SSI when using vancomycin and rifampicin compared with cefazolin, which suggests that this is the dominant strategy. The study did not consider the impact of antibiotic resistance on the long-term effectiveness of antibiotic prophylaxis.

Zanetti and colleagues ${ }^{37}$ undertook a costeffectiveness analysis and a cost-utility analysis based on a decision analytic approach, informed by data in the published literature. A statetransition model was used for the reference case analysis to incorporate the lifetime probability of death, myocardial infarction, angina or asymptomatic coronary artery disease following CABG to estimate life expectancy, quality-adjusted life expectancy and total lifetime costs.

The use of five doses of $1 \mathrm{~g}$ of vancomycin administered over 48 hours for perioperative prophylaxis against SSI in patients undergoing CABG was compared with six doses of cefazolin over 48 hours. The analysis was undertaken in the USA and the base case analysis was reportedly undertaken from the societal perspective, although there was no inclusion of productivity losses. The reference case was undertaken from the healthcare payer perspective. The study population for the base case analysis comprised a hypothetical cohort of 10,000 patients who underwent CABG. The reference case analysis was based on a 65-year-old man with stable, multi-vessel coronary heart disease. It was not clear whether surgery was elective. The length of follow-up was 3 months post-surgery for the base case and 5 years for the reference case.

The base case analysis measured outcomes using the number of deep SSIs avoided, the number of 
superficial SSIs avoided and the number of hospital deaths averted. The reference case analysis used quality-adjusted life-years (QALYs). Additionally the following outcomes were measured: incidence of causative organisms, the RR of SSI caused by susceptible organisms, the incidence of antibiotic-related AEs, the incidence of SSI due to resistant organisms and the probability of hospital death.

The authors provide a table which reports on the model variables; however, some of the data are difficult to interpret since it is not clear whether they are probabilities or rates. The incidence of superficial SSI was 0.08 (plausible range $0.02-0.12$ ). The incidence of deep SSI was 0.04 (plausible range 0.01-0.06). The RR of SSI caused by susceptible organisms was estimated to be the same for vancomycin versus no prophylaxis and cefazolin versus no prophylaxis at 0.4 (plausible range $0.2-0.8$ ). It is not clear if these figures refer to a rate.

The organisms causing SSI were S. aureus 0.25 (plausible range 0.2-0.35), CNS 0.25 (plausible range $0.2-0.35$ ), enterococci 0.05 (plausible range $0.02-0.15)$ and Gram-negative bacteria 0.3 (plausible range $0.15-0.5$ ). It seems that these data are probabilities, but it is not clear.

The incidence of SSI due to resistant organisms was 0.012 (plausible range $0-0.03$ ) for MRSA (as a proportion of all SSIs due to $S$. aureus), 0.024 (plausible range 0-0.03) for MR-CNS (as a proportion of all SSIs due to CNS), 0.003 (plausible range 0-0.006) for VRE (as a proportion of all SSIs due to enterococci) and 0.01 (plausible range 0-0.036) for cefazolin-susceptible Gramnegative bacteria (as a percentage of all SSIs due to Gram-negative bacteria). A history of allergy to $\beta$-lactams was estimated at 0.1 (plausible range $0.05-0.15)$. The incidence of vancomycin-related AEs was 0.08 (plausible range 0.01-0.2) and the incidence of cefazolin-related AEs was 0.08 (plausible range $0.01-0.2$ ). The probability of hospital death due to deep SSI was 0.082 (plausible range 0.01-0.1), due to an antibiotic allergic reaction 0.00002 (no range provided) and due to CABG surgery-related events 0.036 (plausible range 0.01-0.1).

For the reference case, QALYs were estimated by applying weights to the health states representing death, myocardial infarction, angina or asymptomatic coronary artery disease. Quality weights were obtained from the Beaver Dam Health Outcomes Study, in which the time trade- off technique was used to elicit utilities. ${ }^{38}$ It was not stated who provided these valuations. A wide range of quality weights for temporary health states were explored for superficial and deep SSI.

For the base case, it was found that if no antibiotic prophylaxis was given, 570 patients would develop a deep SSI and for every 10,000 patients there would be 405 hospital deaths. Routine use of vancomycin would result in 368 deep SSIs and 388 hospital deaths per 10,000 patients. Routine use of cefazolin would result in 397 deep SSIs and 391 hospital deaths per 10,000 patients.

For the reference case, use of routine vancomycin would result in a gain of 8.339 QALYs for the 65-year-old male, compared with 8.335 QALYs for use of cefazolin. The incremental QALY for cefazolin versus no prophylaxis was 0.023 and the incremental QALY for vancomycin versus cefazolin was 0.004 .

Costs included the use of the antibiotics and perfusion, SSI, hospital deaths, medical charges and AEs and were obtained from the published literature. Unit costs were not reported and neither was resource use. Future costs (and benefits) were discounted at an annual rate of $3 \%$.

For the base case, if no antibiotic prophylaxis was used, the total cost per 10,000 patients was US $\$ 33,410,000$ (1998 price year). US $\$ 23,360,000$ of vancomycin was administered and US $\$ 24,530,000$ if cefazolin was administered. It was calculated that an incremental saving of US $\$ 8,880,000$ per 10,000 patients would be gained from using cefazolin instead of no antibiotic prophylaxis and US $\$ 1,170,000$ per 10,000 patients from using vancomycin rather than cefazolin.

For the reference case, the lifetime cost was US $\$ 62,892$ if no antibiotic prophylaxis was administered and US $\$ 61,913$ when administering vancomycin and US $\$ 62,016$ using cefazolin prior to surgery. The use of cefazolin rather than no antibiotic prophylaxis resulted in an incremental saving of US $\$ 876$. The use of vancomycin rather than cefazolin resulted in an incremental saving of US\$103.

Costs and benefits were not synthesised. The cefazolin strategy dominated the no prophylaxis strategy. The vancomycin strategy was as cost saving and as effective as the cefazolin strategy. The authors suggested that there may be a tradeoff between short-term benefits to individual 
patients and long-term consequences for society in terms of increased antibiotic resistance. Due to a lack of data on the societal consequences that might result, the authors were reluctant to recommend a change in practice.

Univariate and multivariate sensitivity analysis was undertaken to explore the impact of variability on the estimates used in the model. Sensitivity analysis was conducted on the plausible ranges that were used as parameters for the analysis. Additionally, the potential variation in the cost of the antibiotics and the cost of administering them, and the use of different SSI rates, were explored in sensitivity analyses. The sensitivity analyses showed that no prophylaxis was always the most costly and the least effective option. The ranking of vancomycin compared with cefazolin was not affected by the sensitivity analyses. Results were most sensitive to changes in the cost of vancomycin, its efficacy and that of cefazolin, and the prevalence of bacterial resistance to antibiotics.

The authors did not model the relationship between antibiotic prophylaxis and resistance, due to lack of data. However, they did conduct sensitivity analyses to explore the impact of a decrease in efficacy of vancomycin on study results. They argued that the routine use of vancomycin would remain more effective and less costly than the routine use of cefazolin if all enterococci were resistant to vancomycin because of the small proportion of SSIs caused by enterococci postoperatively. They simulated a hypothetical scenario in which prevalence of VRE would continue to increase by $2 \%$ per year, as reported in US hospitals from 1989 to 1997, and assumed the same trend for staphylococci. They assumed that vancomycin prophylaxis, but not cefazolin prophylaxis, would accelerate this trend by $50 \%$. The result was that after 6 years vancomycin was no longer less costly than routine cefazolin and would become less effective over 13 years. However, this result was based on author assumption about resistance rates and they argued that no conclusions could be drawn from it.

\section{Discussion}

Four out of five of the studies focused on condition-specific measures of effect such as the rate of postoperative prosthetic and wound infections and AEs including mortality and sideeffects. ${ }^{13,22,35,36}$ Zanetti and colleagues included a generic measure of preference-based healthrelated quality of life, based on time trade-off valuations. ${ }^{37}$ For the reference case, QALYs were calculated, as favoured by NICE

(http://www.nice.org.uk/). ${ }^{39}$

Three studies calculated ICERs and undertook sensitivity analyses, ${ }^{13,35,37}$ only one of which included multivariate sensitivity analysis. ${ }^{37} \mathrm{No}$ studies undertook probabilistic sensitivity analysis.

Only one of the studies explored the implications of antibiotic resistance in any detail, ${ }^{37}$ although two of the other studies noted the bacteriological organisms found in existing SSI. ${ }^{22,36}$ Zanetti and colleagues undertook an exploratory sensitivity analysis to assess the impact of resistance in terms of a reduction in the efficacy of vancomycin on the study results. 37

\section{Summary}

The conclusions from this review are as follows:

- Only one study incorporated health-related quality of life and undertook a cost-utility analysis, the approach favoured by NICE.

- SSI can increase patient length of stay considerably, which has substantial cost implications. The length of hospital stay is the key cost driver from the secondary care perspective.

- None of the studies was undertaken in the UK, limiting the transferability of the results to the UK setting.

- No studies explicitly modelled antibiotic resistance as part of the primary analysis. 



\section{Chapter 5}

\section{Supplementary economic reviews}

\section{Introduction}

This chapter provides details of a series of literature reviews relating to issues of costeffectiveness in the context of the management of MRSA. In addition to conducting a systematic review of the effectiveness evidence on glycopeptide antibiotics compared with nonglycopeptide antibiotics, the research brief was to conduct a review of the corresponding costeffectiveness evidence to inform the development of an economic model. A key objective was to assess the impact of antibiotic prophylaxis on MRSA infection rates in surgical patients and to explore the potential longer term consequences the use of these antibiotics may have in terms of increased antibiotic resistance. To address this, four supplementary reviews were undertaken to broaden the scope of the review and to expand the potential pool of data that were available for use in developing the economic model. Details of the literature searches for these supplementary reviews are provided in Appendix 1.

The first supplementary review assessed the published economic evaluation literature which compared the costs and effects associated with the use of any antibiotic prophylaxis for surgery, excluding glycopeptides. The purpose of this review was to characterise the methods used in economic evaluations of antibiotic prophylaxis for surgery, including interventions other than glycopeptides. This review could provide useful information about how to conduct evaluations in this field and it provided the opportunity to use indirect evidence to strengthen the inference concerning the relative efficacy of the treatments for comparison. The cost-effectiveness review in Chapter 4 assessed studies that made direct comparisons with glycopeptides - that is, it included evaluations of glycopeptides compared with other antibiotics (e.g. interventions A versus $B$ and $B$ versus $C$, where $B$ was a glycopeptide and $\mathrm{A}$ and $\mathrm{C}$ were non-glycopeptides). This review included studies that could be used as indirect evidence (e.g. studies comparing interventions A with $\mathrm{C})$.

A second supplementary cost-effectiveness review was undertaken to assess economic evaluations of antibiotics where antibiotic resistance is a problem and, therefore, looked beyond antibiotic prophylaxis used in surgery. The purpose of this review was to explore the methodological approaches used to evaluate the antibiotic resistance problem.

A further two selective reviews were undertaken to enhance the development of an economic model. The first of these explored the literature on the use of epidemiological and decision analytic models in infectious diseases where resistance to antibiotics is an issue. The purpose was to obtain examples of methodological approaches to assessing and modelling the impact of antibiotic resistance in cost-effectiveness analyses. The final review explored the published literature to develop a conceptual evaluative framework for the economic evaluation of policies against MRSA. It considers different dimensions of the evaluation problem and a number of methodological approaches which might be applied to deal with these problems.

\section{Economic evaluations of non-glycopeptide antibiotic prophylaxis for surgery}

\section{Methods \\ Inclusion criteria}

Studies that were full economic evaluations were considered: (1) cost-effectiveness evaluations, including cost-minimisation and cost-consequence analyses; (2) cost-utility analyses; and (3) cost-benefit analyses. ${ }^{34}$ Comparator interventions had to include non-glycopeptide antibiotic prophylaxis for surgery.

\section{Data extraction and quality assessment}

Data were extracted on key components of standard economic evaluations including data on the comparators, study population, the length of prophylaxis, the type of surgery, the setting and perspective, the costs and outcomes measured, the definition of infection used, details of adverse drug reactions, the length of follow-up, estimates of incremental cost-effectiveness, how antibiotic resistance was handled, the risk of confounding, 
costs attributable to infection and details of sensitivity analyses.

\section{Results}

Key methodological issues in this area, apart from general economic evaluation method quality criteria, are the use of appropriate comparators, study population, the length of prophylaxis, the type of surgery, the costs and outcomes measured, the definition of infection used, details of adverse drug reactions, the length of follow-up, how antibiotic resistance was handled, the risk of confounding and methods used to determine costs attributable to infection. The studies were examined for any helpful lessons learnt that could be applied to the economic evaluation of glycopeptides, in addition to the economic evaluations of glycopeptides reviewed in Chapter 4.

Forty-three studies were obtained from the searches. Of these, 23 economic evaluations of antibiotic prophylaxis were reviewed. However, one economic evaluation was excluded because it was in Japanese and there was no English version of the abstract. ${ }^{40}$

Of the remaining 22 economic evaluations, 12 were RCTs. Eight were based on non-randomised cohorts and two were secondary economic evaluations, using meta-analyses.

The surgical groups were cardiothoracic (3), ear, nose and throat (ENT) (1), orthopaedic (2), abdominal and gynaecological (5), vascular (1), urological (2), head and neck (2), maxillofacial (1), penile prosthesis implants (1), breast (1), multiple surgical groups (1), inguinal hernia repair (1) and appendectomy (1). Only four trials had fewer than 100 patients.

Oral, intravenous and topical antibiotics were evaluated, but most studies examined the use of intravenous antibiotics only. Table 10 provides details of the prophylaxis regimens assessed by the evaluations and Table 11 provides full details of all the included studies.

The length of prophylaxis was not always stated, but ranged from one dose preoperatively, to 7-day courses of antibiotics. Adverse drug reactions (ADRs) were not usually reported, and when they were reported there was a wide variation. ADR rates reported by Paladino and colleagues comparing ampicillin-sulbactam with cefoxitin were around 20\%. ${ }^{58}$ Other studies reported much lower rates.

\section{Discussion}

The main outcomes used were condition specific: wound and SSIs, although variable definitions were applied. Diagnosis was usually based on clinical criteria, although some also included microbiological culture. Some studies appeared to differentiate between joint, deep and superficial/incisional wound infections. No generic measures of quality of life (QoL) or morbidity data were reported other than impact on length of hospital stay.

Costs were poorly reported in most studies. Two studies did not report actual costs at all ${ }^{41,45}$ and seven only reported costs for the antibiotics. ${ }^{46,53,55,58-60,62}$ The remaining studies combined the cost of the antibiotics with locally determined or national tariff charges for increased

TABLE 10 Types of antibiotic prophylaxis compared in the economic evaluations

\begin{tabular}{|c|c|c|}
\hline Study & Combinations & Number of studies \\
\hline $\begin{array}{l}\text { Pearson, 1996; } ;^{41} \text { Blair, 1995; } ;^{42} \text { VandenBergh, } 1996 ; ;^{43} \\
\text { Pearle, 1997; }{ }^{44} \text { Schilling, 1997; }{ }^{45} \text { Wieck, } 1997 ;{ }^{46} \text { Bold, 1998; }{ }^{47} \\
\text { Kapoor, 1998; } ;^{48} \text { Kulling, 2000; } ;^{49} \text { Lazorthes, } 1992^{50}\end{array}$ & Active versus placebo & 10 \\
\hline Albers, $1994^{51}$ & $\begin{array}{l}\text { One or more of the comparators } \\
\text { not stated }\end{array}$ & 3 \\
\hline $\begin{array}{l}\text { Davey, } 1995 ;{ }^{52} \text { Pestotnik, } 1996 ; ;^{53} \text { Schwartz, } 1996 ;{ }^{54} \\
\text { Pavan, } 1992^{55}\end{array}$ & $\begin{array}{l}\text { One or more of the comparators } \\
\text { a combination of more than one } \\
\text { prophylaxis regimen }\end{array}$ & 4 \\
\hline Thomas, $1999 ;{ }^{56}$ Rau, $2000^{57}$ & Comparison of two cephalosporins & 2 \\
\hline $\begin{array}{l}\text { Paladino, } 1994 .{ }^{58} \text { Heit, } 1997 ;{ }^{59} \text { Hall, } 1993 ;{ }^{60} \text { Roach, } 1990 ; ;^{61} \\
\text { Rau, } 2000^{57}\end{array}$ & Cephalosporin versus a penicillin & 5 \\
\hline Fried, $1996^{62}$ & Culture specific & I \\
\hline
\end{tabular}




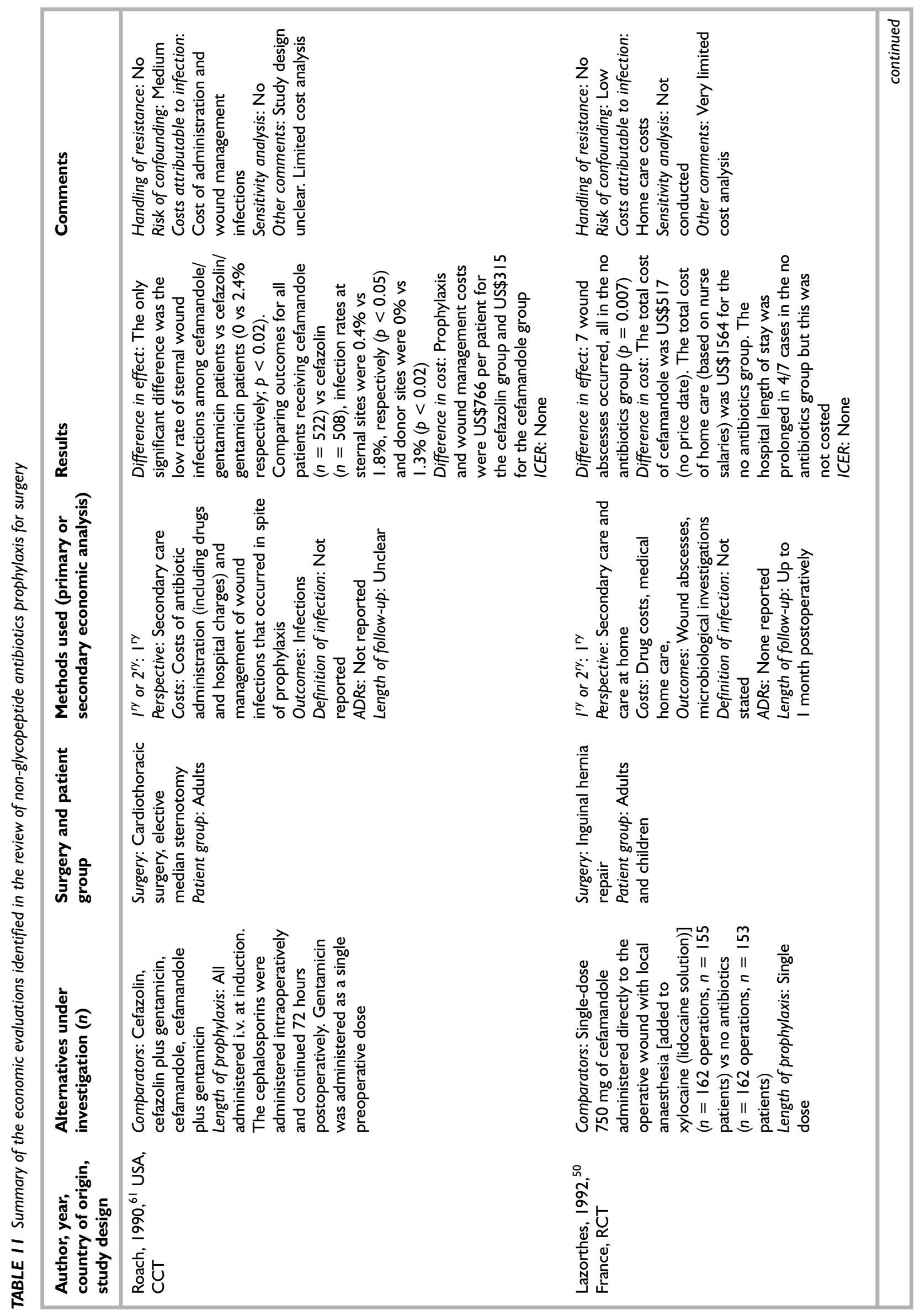




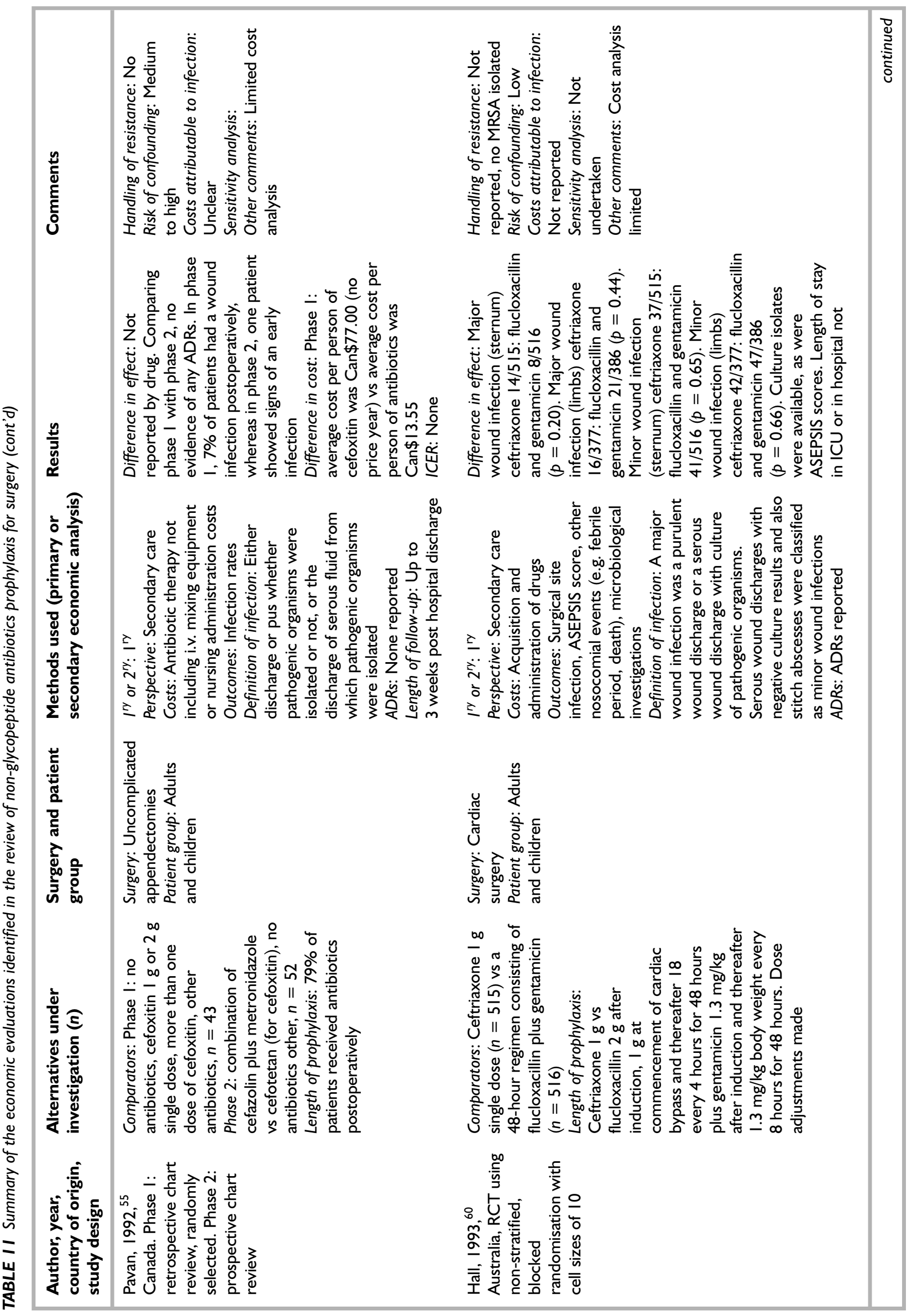




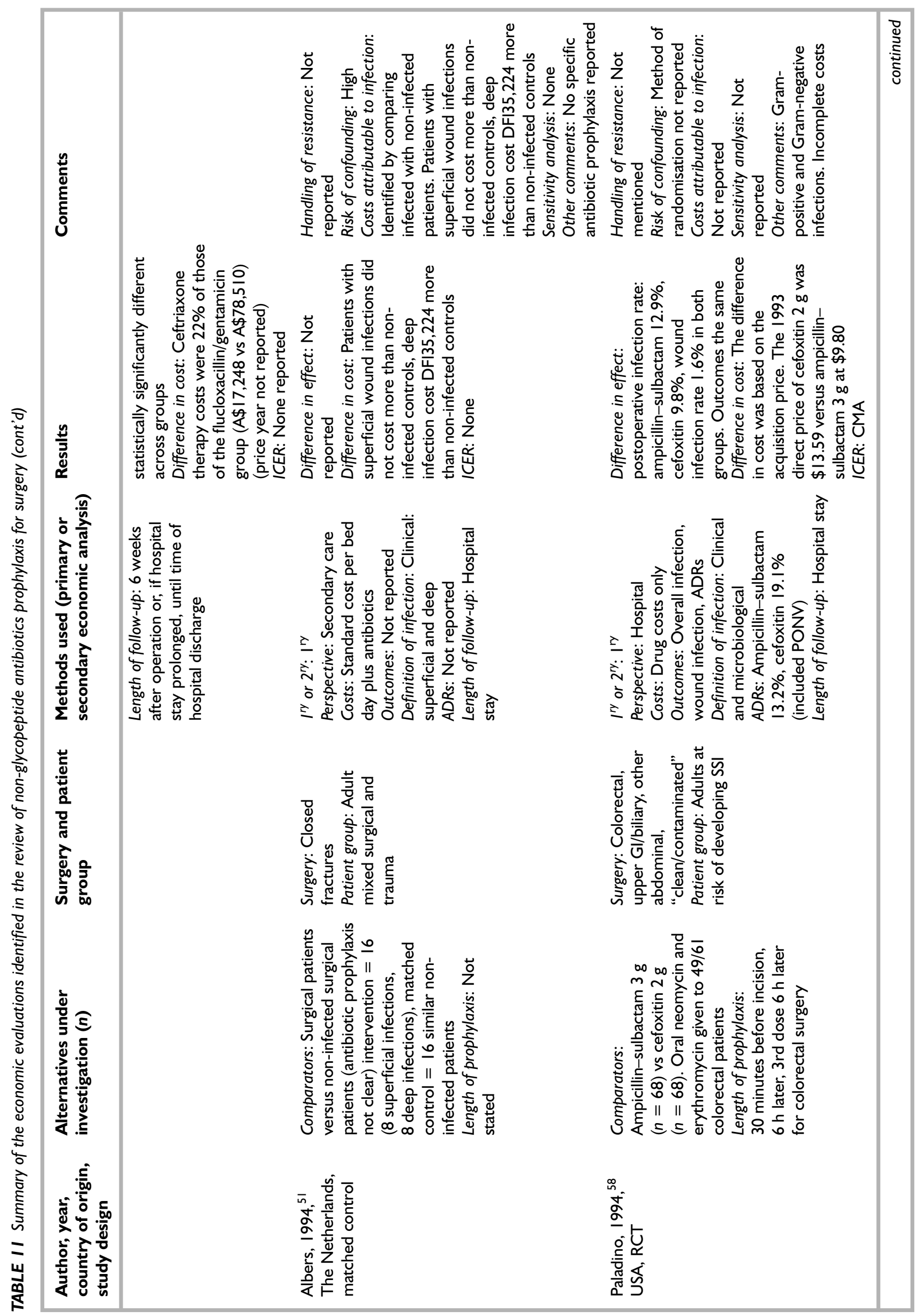




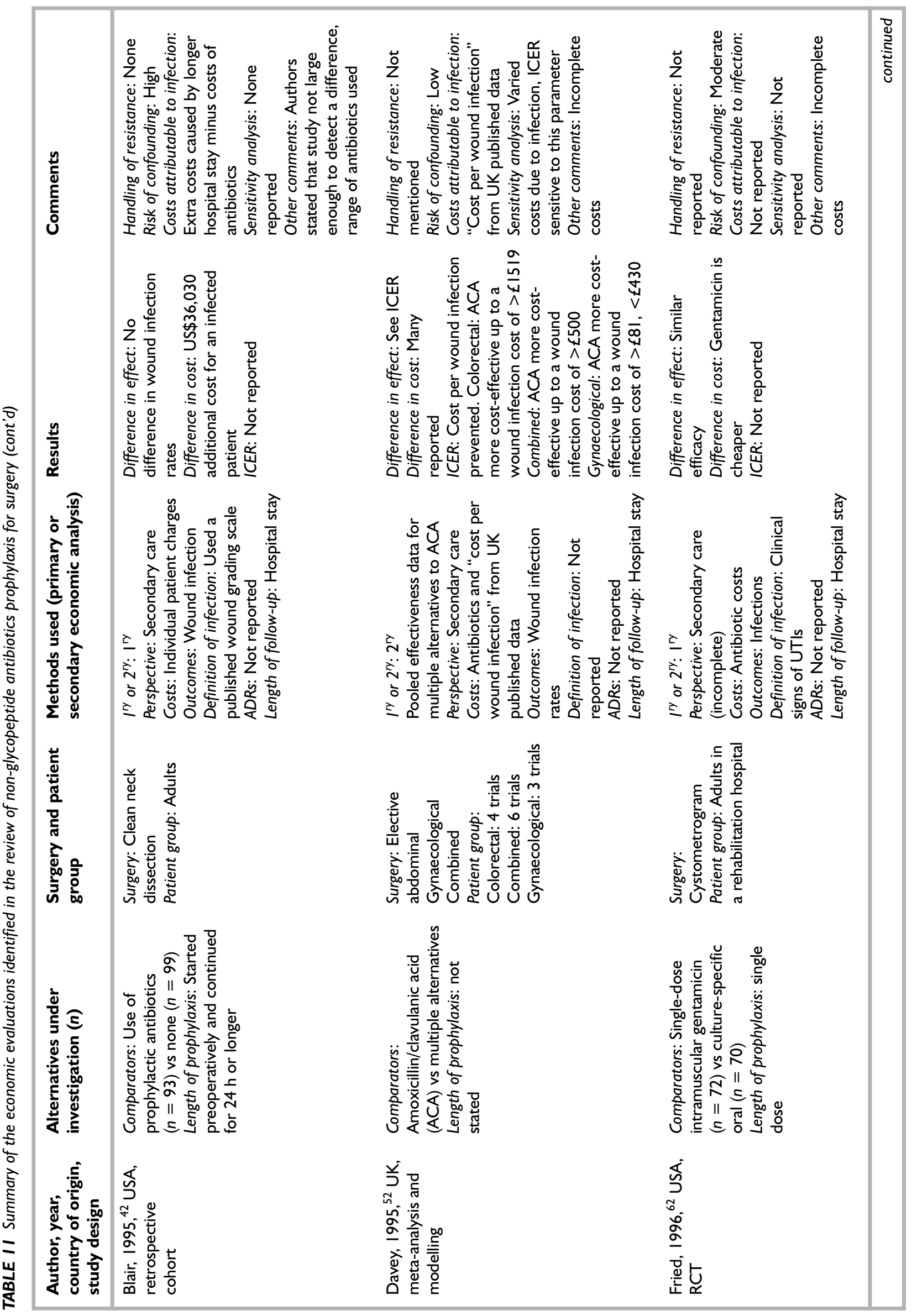




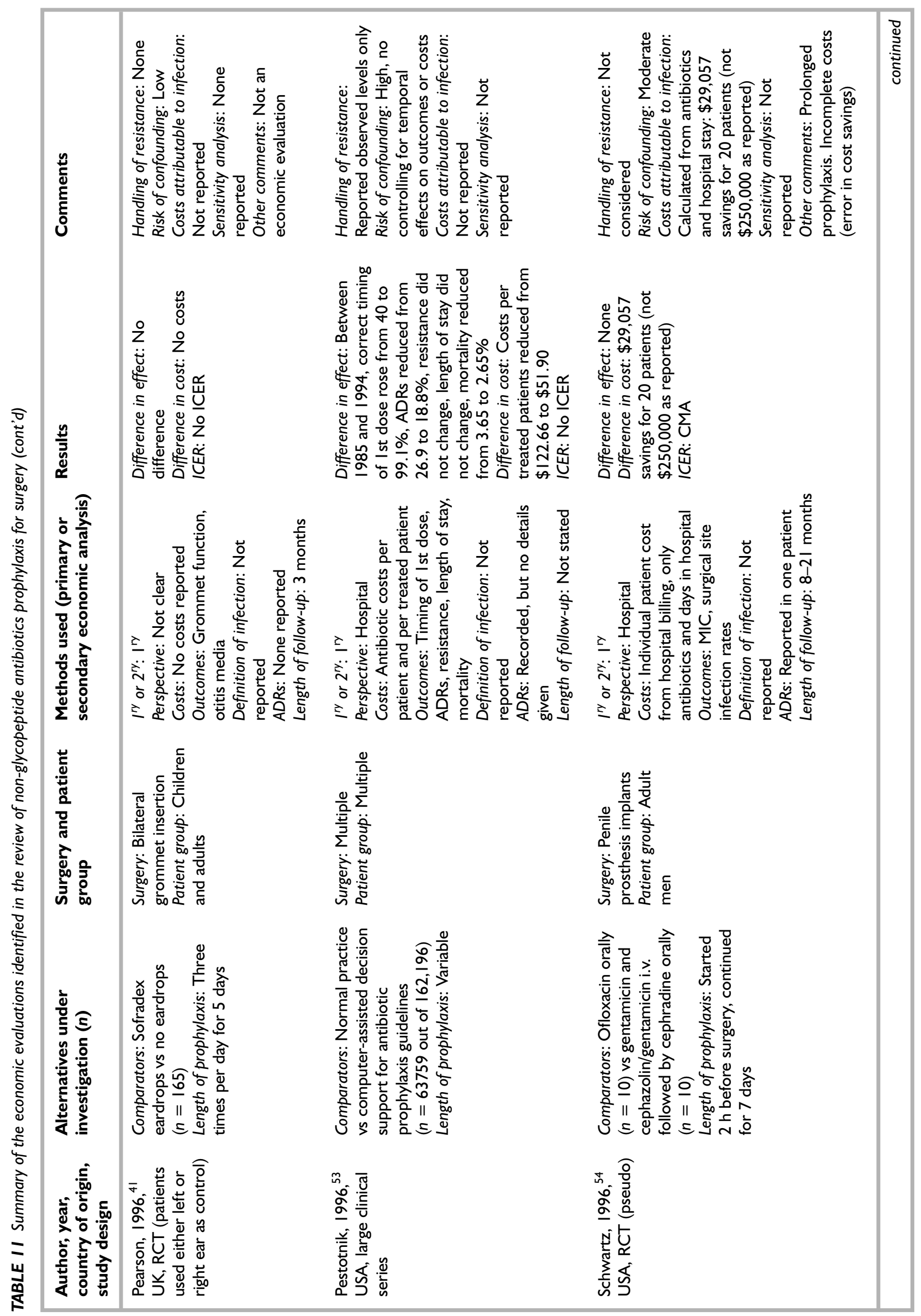




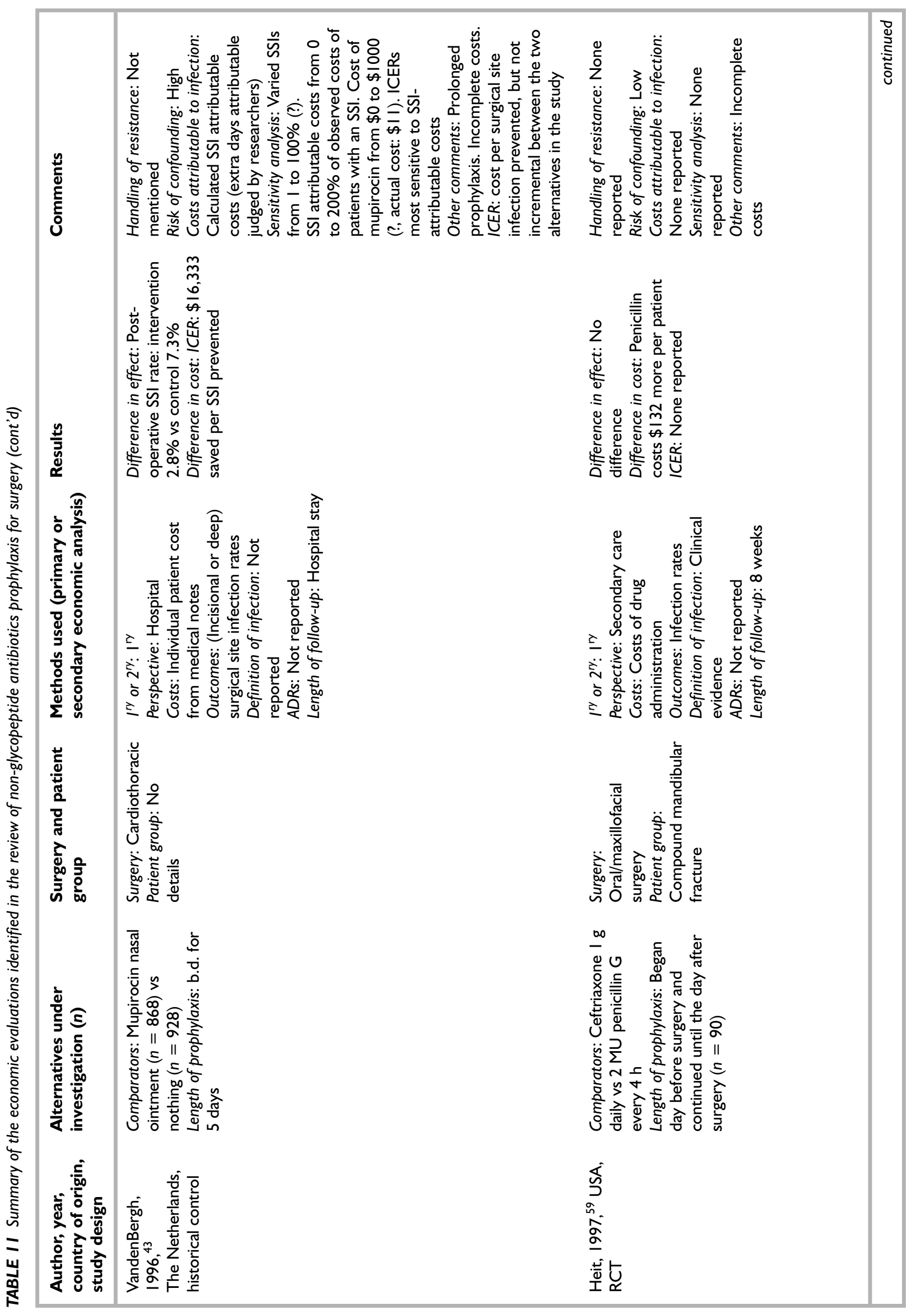




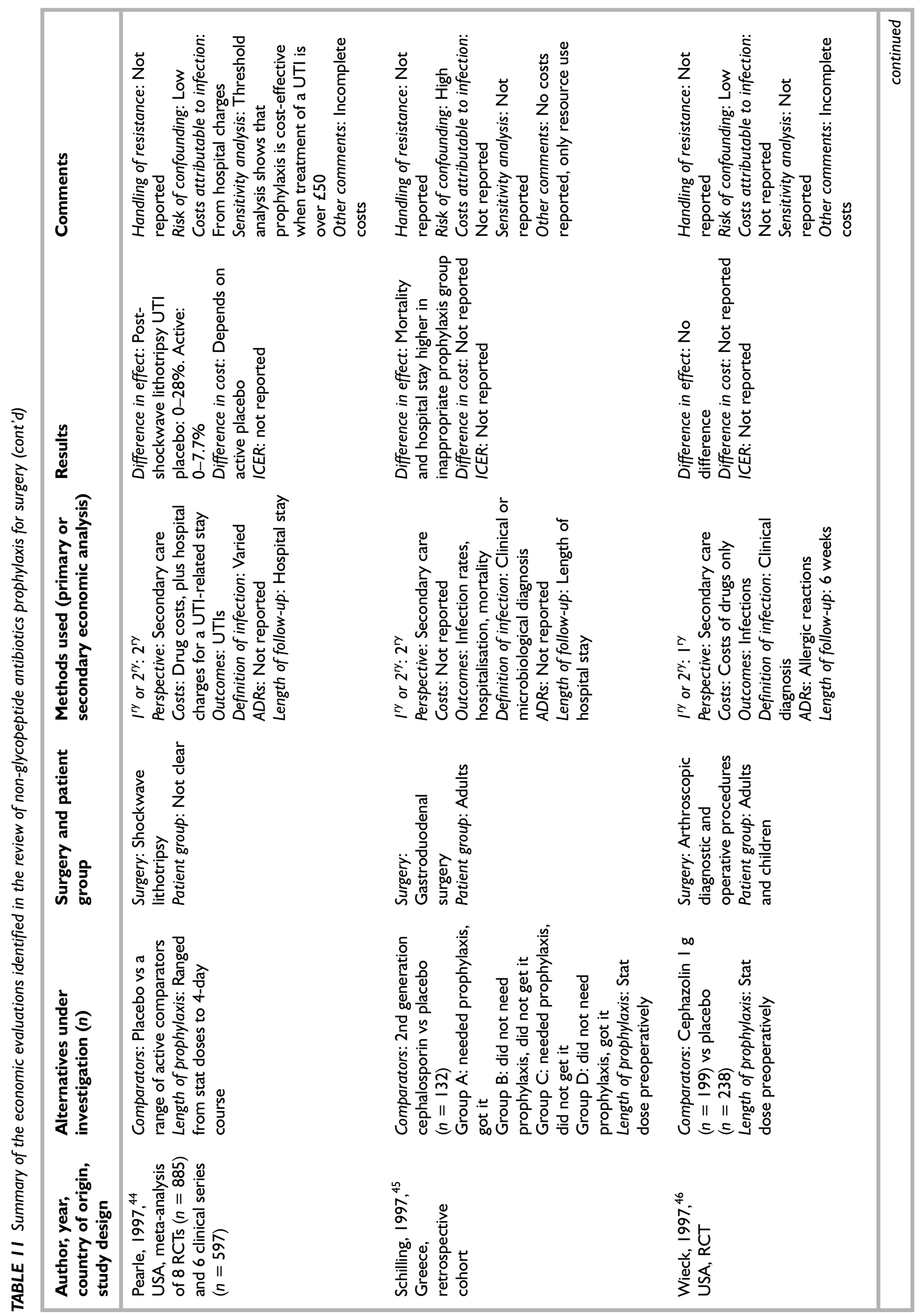




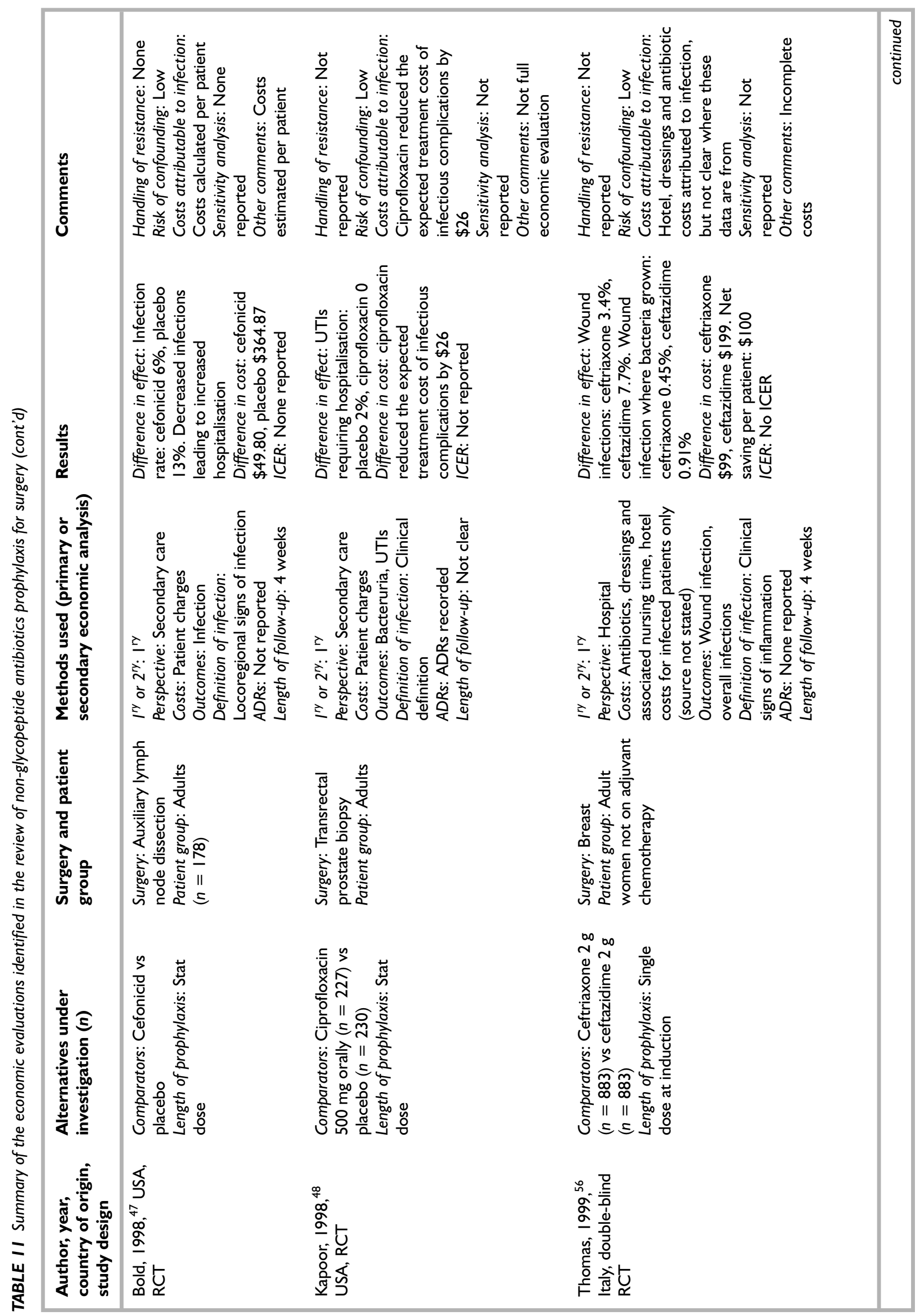




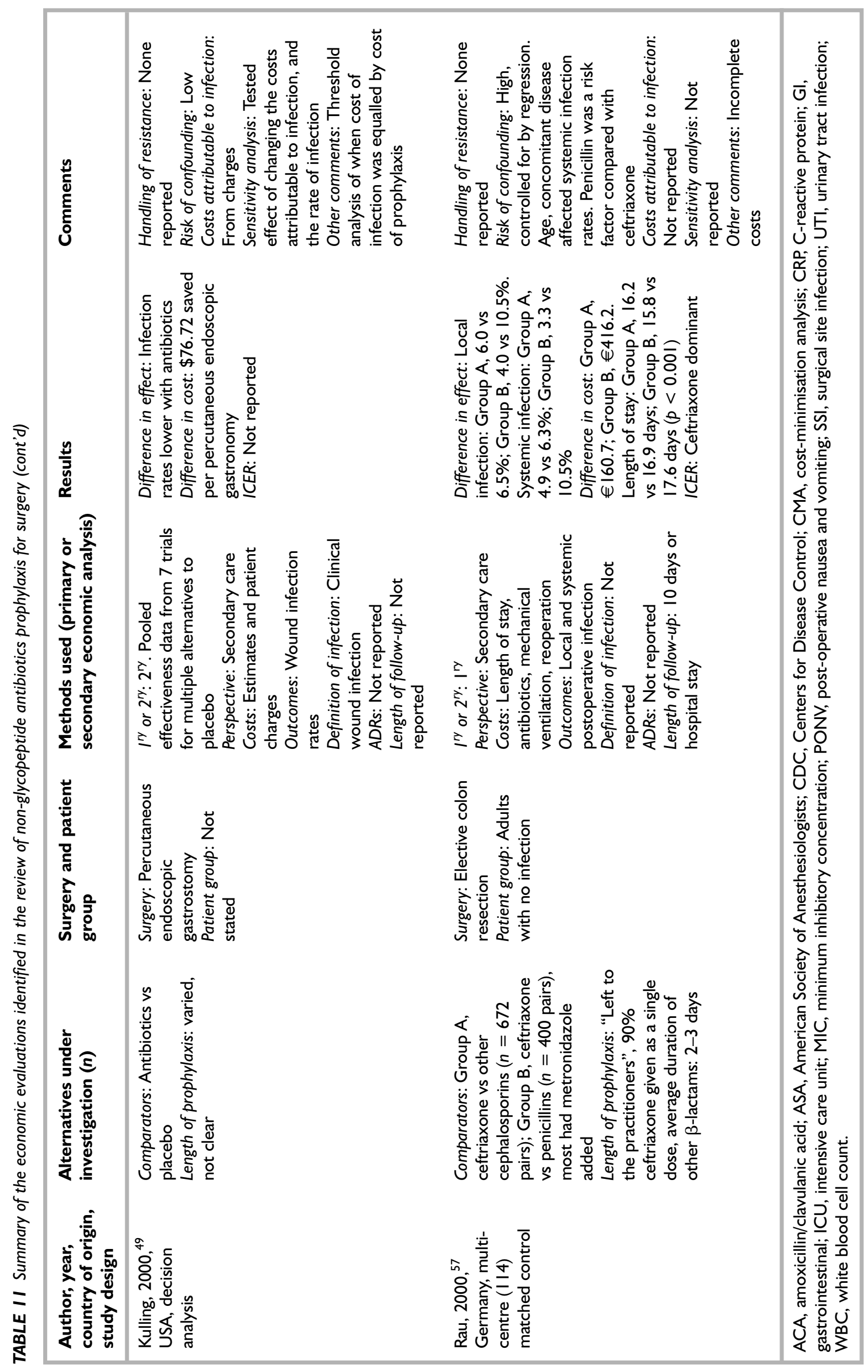


length of stay due to infection. ${ }^{42-44,47-52,54,56,57,61}$ Extra length of stay due to infection is a dominating factor in costs. This was obtained in a range of ways, from direct measurement, use of published data or qualitative assessment about whether days in hospital were attributable to infection or not. The perspective was always the secondary care payer, when it could be determined.

Only four studies reported ICERs. ${ }^{43,44,49,52}$ Only four studies reported sensitivity analysis, which suggested that costs attributable to infection had the most effect on the ICER.

There was no explicit modelling of resistance in any study, in either a static or dynamic model.

\section{Summary}

In summary, the lessons learnt from this review are as follows:

- Outcomes need to be very clearly defined and better reported, including adverse drug events, differing severity of infection and causative organisms.

- The main cost driver (from the secondary care perspective) is length of stay changes caused by infection. This needs to be measured directly and data should be corrected for confounding factors.

- Antibiotic resistance has not been considered explicitly so far.

The impact of surgical infections on costs postdischarge and patient QoL has not been considered so far.

\section{Economic evaluations of antibiotics where antibiotic resistance is a problem}

\section{Introduction}

The purpose of this review was to characterise the methods used to deal with resistance in economic evaluations of antibiotics. Due to the lack of explicit modelling of resistance in economic evaluations in surgical antibiotic prophylaxis, a wider set of studies were examined, including all anti-infective agents.

\section{Methods \\ Inclusion criteria}

Studies that were full economic evaluations were considered, that is: (1) cost-effectiveness evaluations, including cost-minimisation and cost-consequence analyses; (2) cost-utility analyses; and (3) cost-benefit analyses. ${ }^{34}$ Studies evaluated antibiotics and could include evaluations of antibiotic treatment or antibiotic prophylaxis for any medical use.

\section{Data extraction and quality assessment}

Data were extracted on key components of standard economic evaluations including data on the comparators, whether the study was based on primary or secondary economic analysis data, the perspective, the costs and outcomes measured, the length of follow-up, how antibiotic resistance was handled, estimates of incremental cost-

effectiveness and the risk of confounding and any costs attributable to infection.

\section{Results}

Resistance has been examined almost exclusively in the anti-infective management of HIV, malaria and tuberculosis (TB). The studies found were examined to obtain further guidance and ideas as to what methodological approach to employ in seeking to model the impact of antibiotic resistance.

Based on the 43 papers that were obtained through the search undertaken for this review, it was found that resistance appears to be handled differently between different infections. Hence this review is reported by type of infection. This reflects the different mechanisms of resistance, and the relative importance of primary and secondary resistance in treatment failure. Table 12 provides summary details of the included studies.

\section{Discussion \\ HIV}

In HIV, both primary and secondary resistance are key issues in economic evaluations. Resistance develops within an individual patient during treatment, leading to treatment failure and the need to switch to another regimen. Also, treatment-naïve patients can present with resistant HIV strains, referred to as primary resistance. Based on the review, three economic evaluations in HIV consider resistance. All three trials used lifetime Markov modelling. In the two US and one German HIV trials, resistance is only considered as a main component of 'treatment failure', so is considered important in determining whether a patient needs to go on to another regimen, ${ }^{63}$ or the relative cost-effectiveness of genotypic antiretroviral resistance testing. ${ }^{64,65}$ Varying efficacy of HIV highly active anti-retroviral therapy (HAART) from 2 to 50 years (i.e. the effect of change in efficacy due to drug resistance) 


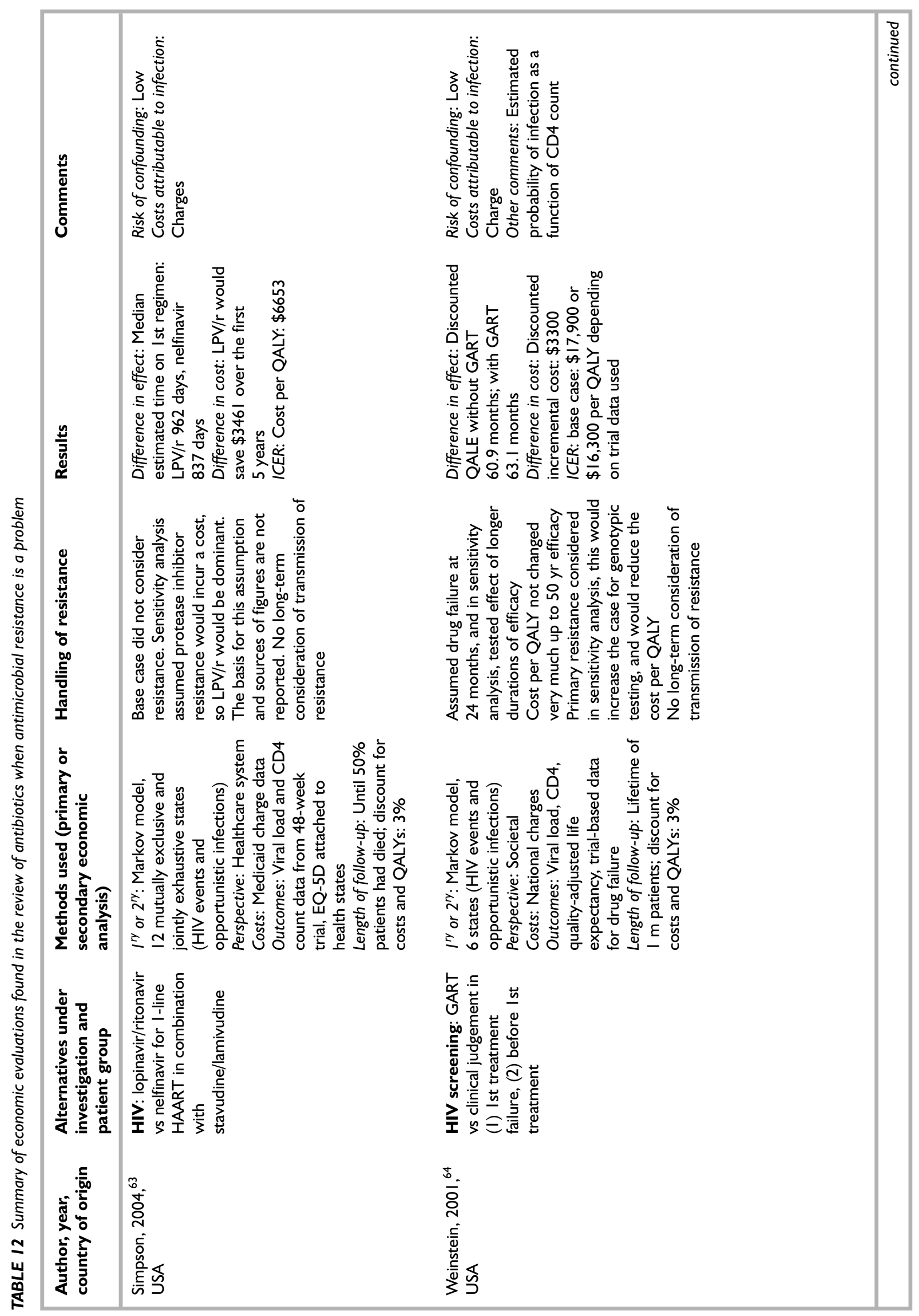




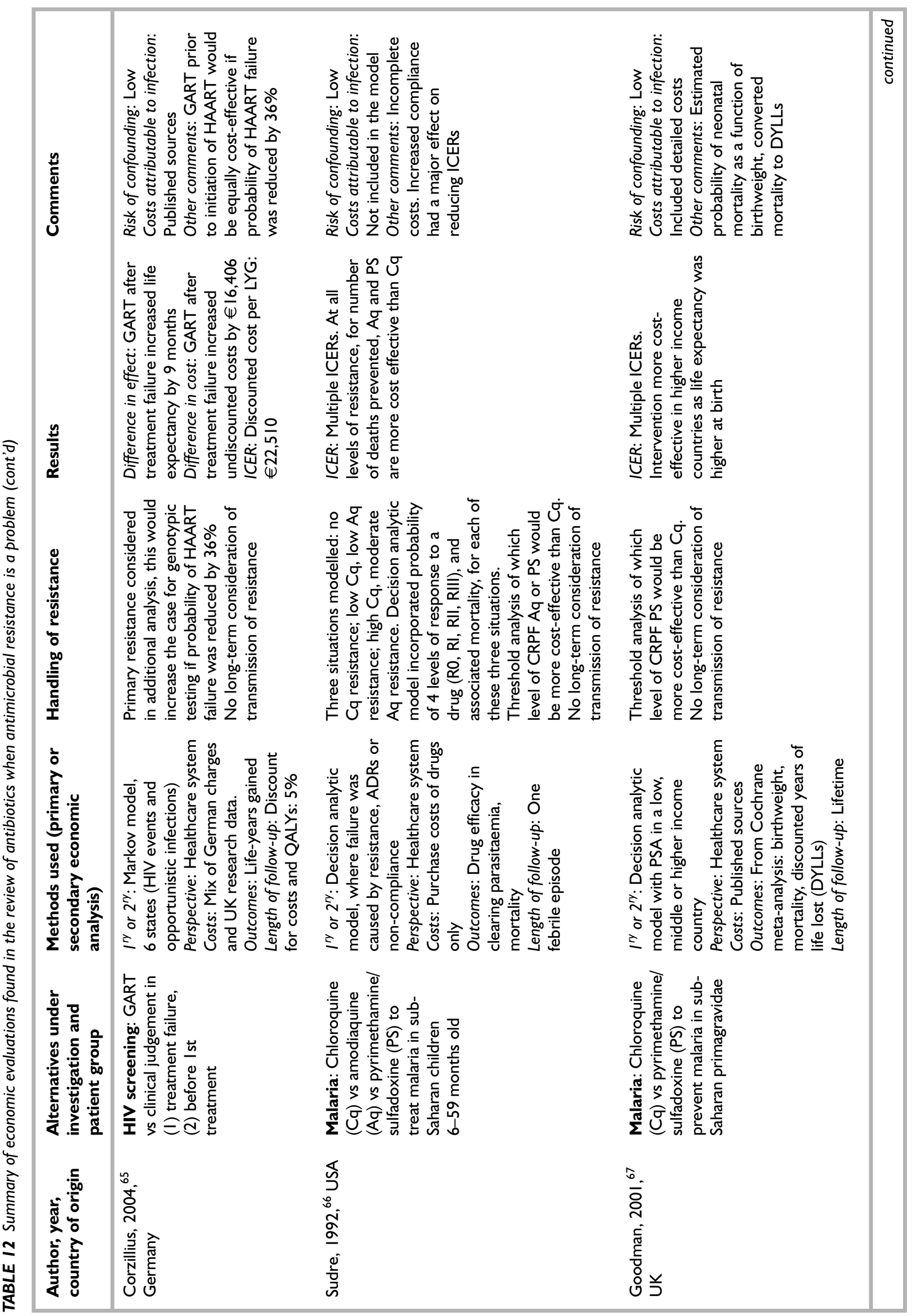




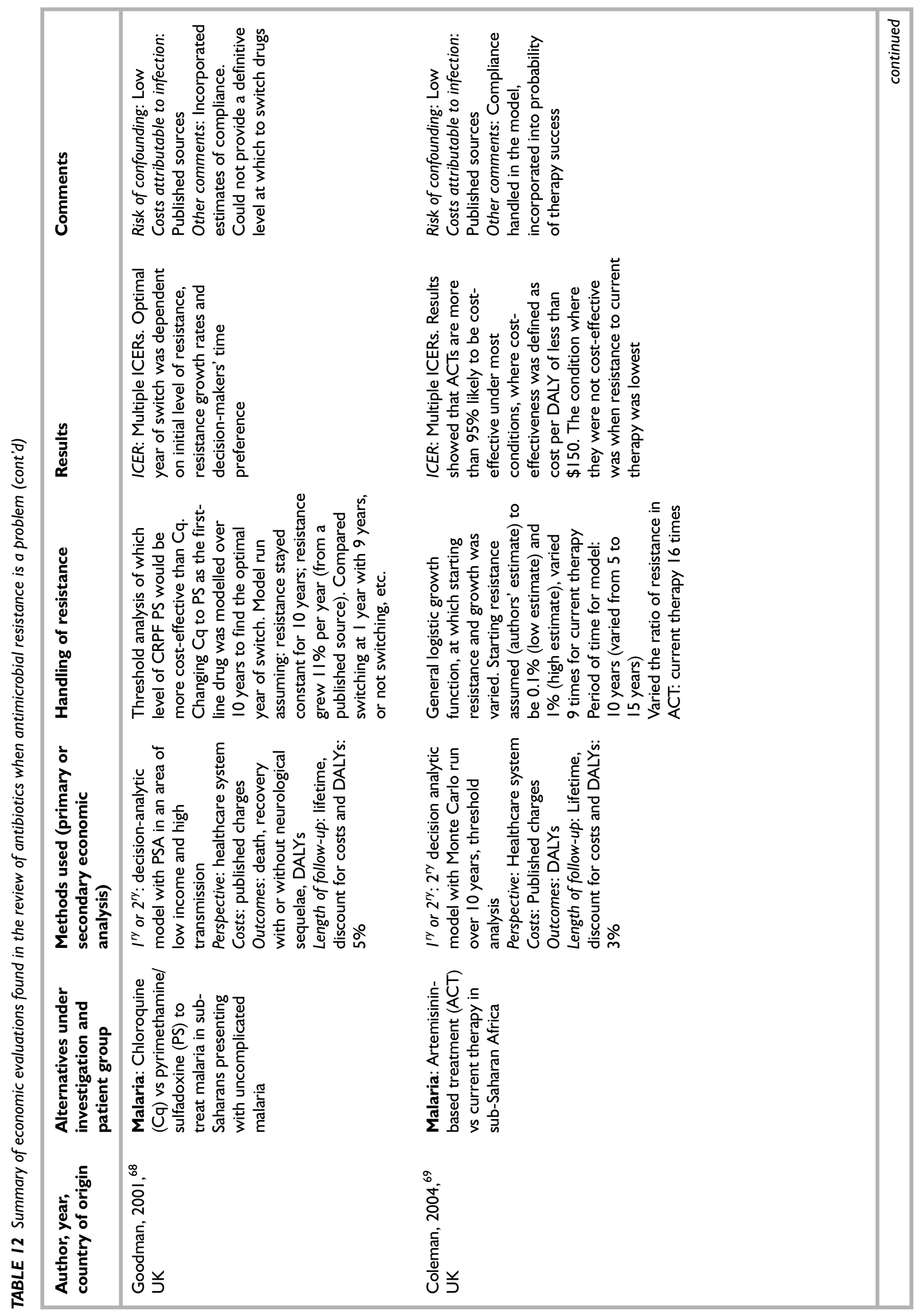




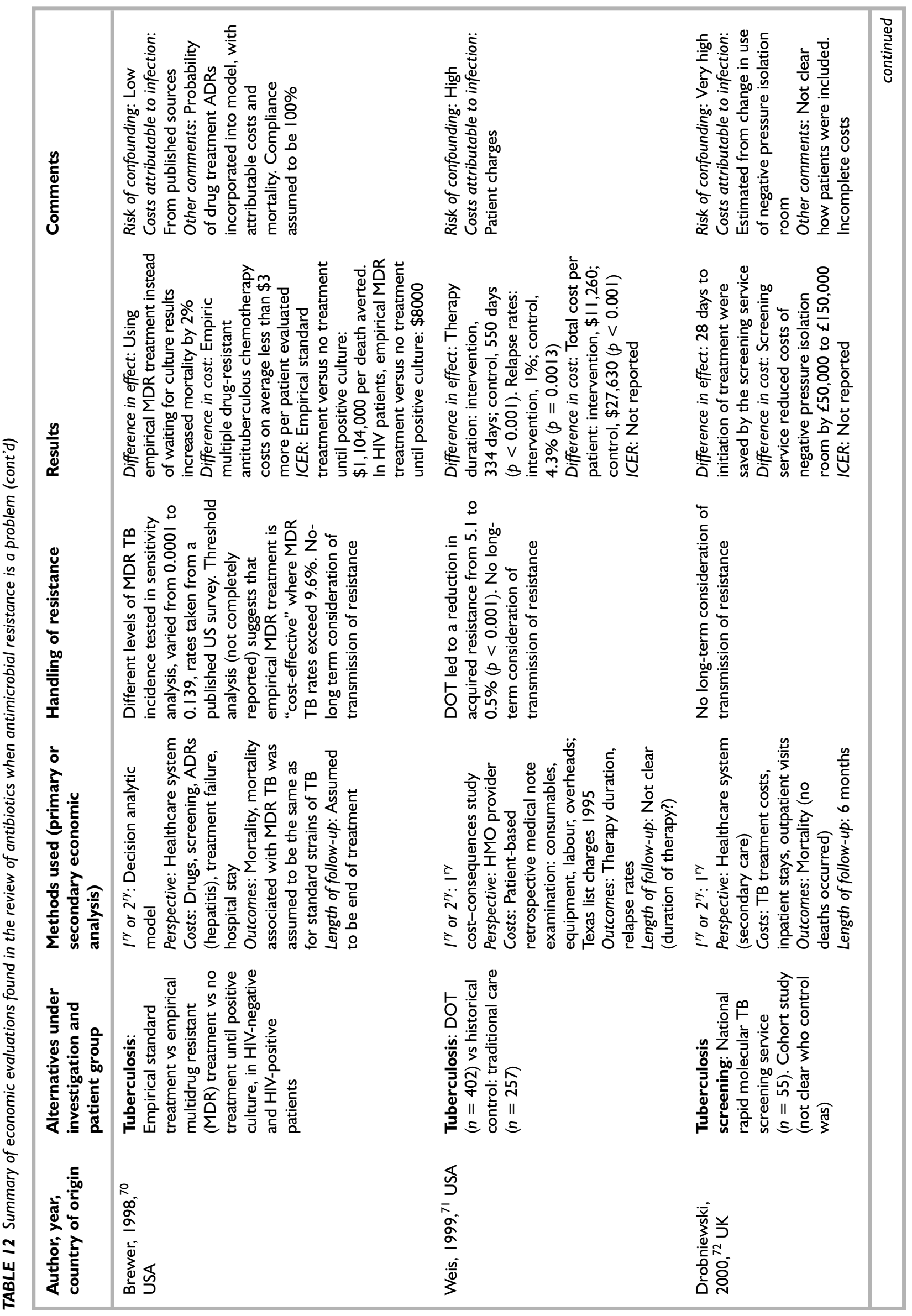




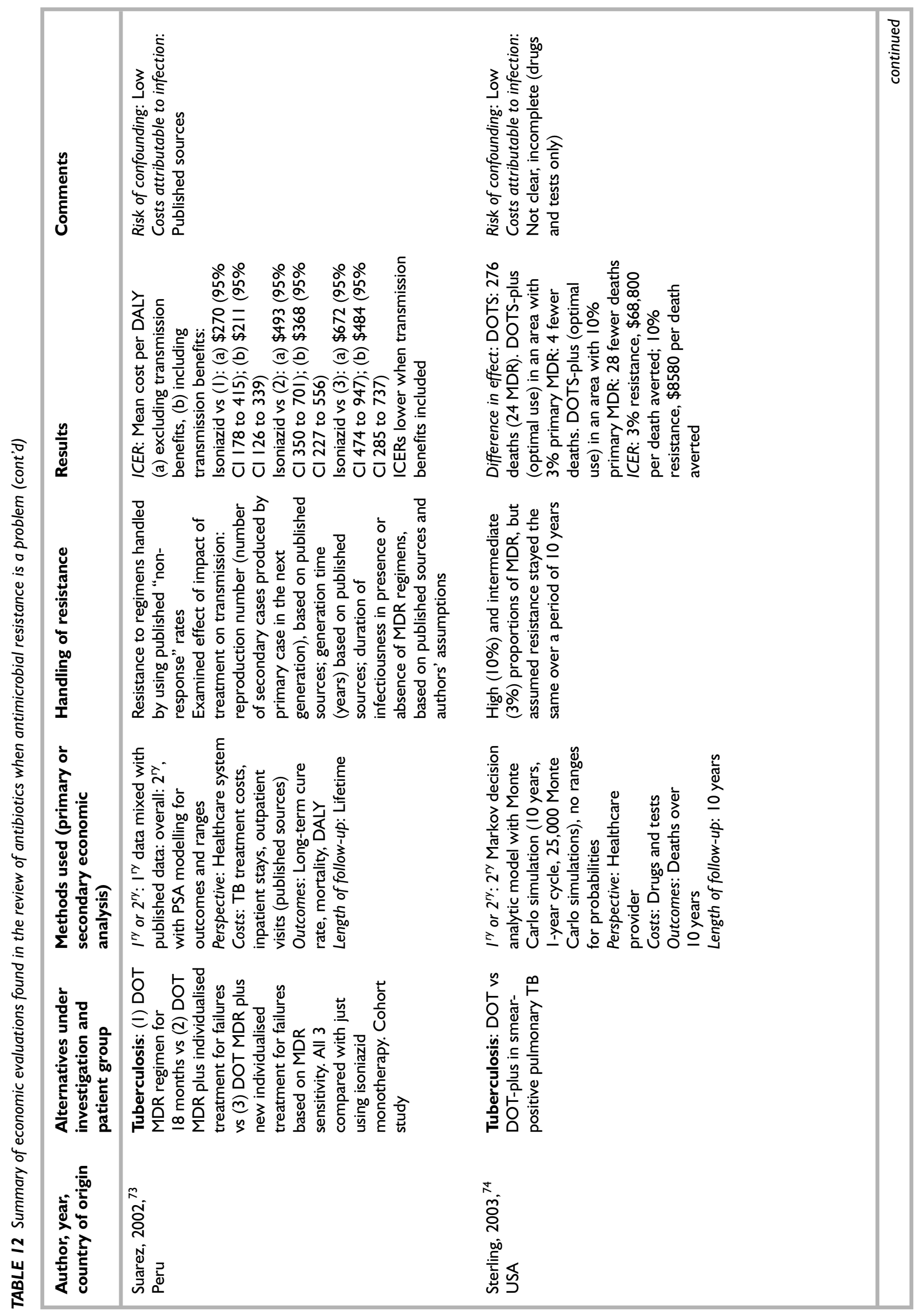




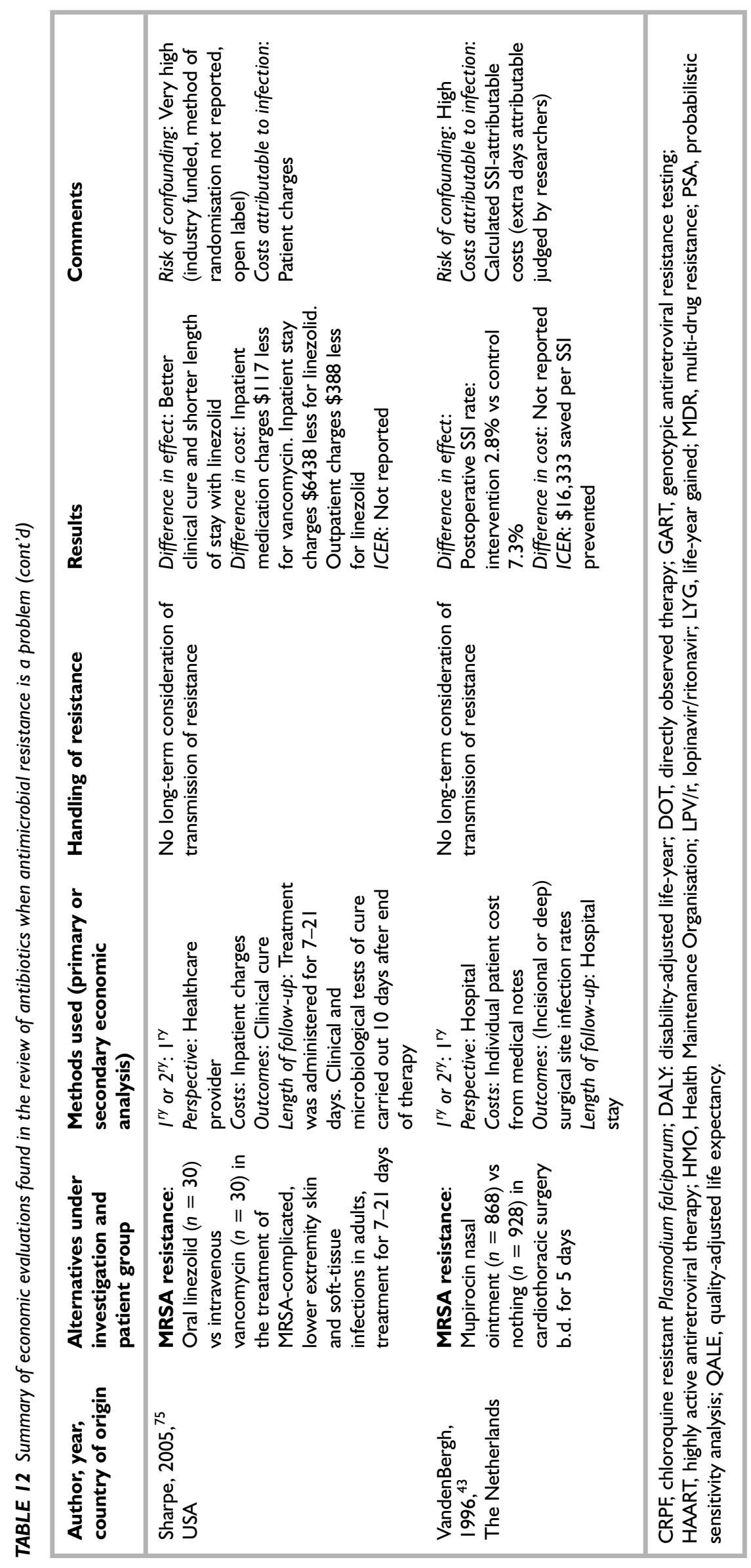


only changed the discounted cost per QALY from US $\$ 17,900$ to US $\$ 19,900 .{ }^{64}$ Primary resistance was considered in Weinstein and colleagues' HIV screening model, in the sensitivity analysis. ${ }^{64}$ This would increase the case for genotypic testing, and would reduce the cost per QALY. It is also considered by Corzillius and colleagues, ${ }^{65}$ to assess a level of resistance at which screening would be important. There is no long term consideration of transmission of resistance in any of these HIV trials, despite the increasing importance of transmission of primary resistance, and associated failure of first-line HAART. ${ }^{63-65}$ There was also no attempt to examine the potential effects of an increase in resistance over time. Compliance was not considered, so was evidently assumed to be $100 \%$, although this is a factor in increasing secondary resistance.

\section{Malaria}

In malaria, the key resistance issue is widespread primary resistance of Plasmodium falciparum to many antimalarial agents. Resistance does not develop within an individual during treatment, but primary resistant strains are transmitted between patients, leading to treatment failure and the need to switch to another regimen. Therefore, resistance is considered as a main component of treatment failure, and levels of resistance in a population predicate empirical first-line treatment. Four economic evaluations in malaria consider resistance. ${ }^{66-69}$

Sudre and colleagues ${ }^{66}$ modelled three levels of drug resistance, and their decision analytic model incorporated the probability of four levels of response to a drug (R0, RI, RII, RIII), and associated mortality, for each of these three situations. They carried out a threshold analysis of which level of chloroquine-resistant Plasmodium faciparum (CRPF) would change which drug was cost-effective. Increased compliance to drugs had a large impact on reducing ICERs. Goodman and colleagues also carried out a threshold analysis of the level of CRPF at which one antimalarial would be more cost-effective than another. ${ }^{67}$

Goodman and colleagues ${ }^{68}$ carried out a threshold analysis to determine at which level of CRPF pyrimethamine/sulfadoxine would be more costeffective than chloroquine. Changing chloroquine to pyrimethamine/sulfadoxine as the first-line drug was modelled over 10 years to find the optimal year of switch. The model was run assuming that (1) resistance stayed constant for 10 years and (2) resistance grew at $11 \%$ per year.
The authors compared switching at 1 year with 9 years, or not switching, and so on, generating multiple ICERs. They were not able to identify an optimal year of switch, as it was dependent on initial level of resistance, resistance growth rates and decision-makers' time preference.

Coleman and colleagues ${ }^{69}$ carried out a threshold analysis to determine at which level of CRPF artemisinin-based combination therapy (ACT) would be more cost-effective than pyrimethamine/ sulfadoxine. Changing pyrimethamine/sulfadoxine to ACT as the first-line drug was modelled over 5, 10 and 15 years. A general logistic growth function was used to calculate the effect of increasing resistance over time, and starting resistance and growth were varied in the model. Starting resistance was assumed (authors' estimate) to be $0.1 \%$ (low estimate) and $1 \%$ (high estimate), and was varied nine times for pyrimethamine/sulfadoxine. The authors also varied the ratio of resistance in ACT to pyrimethamine/sulfadoxine 16 times. The results showed that ACT is more than $95 \%$ likely to be cost-effective under most conditions, where cost-effectiveness was defined as cost per disabilityadjusted life-year (DALY) of less than US $\$ 150$. The condition where they were not cost-effective was when resistance to pyrimethamine/sulfadoxine was lowest.

There was no long-term consideration of transmission of resistance in any of the malaria studies. There was also no attempt to examine the potential effects of an increase in resistance over time in two studies. ${ }^{66,67}$ Two studies attempted to predict the future trajectory of drug resistance, but rates of growth of resistance were assumptive rather than empirical data. ${ }^{68,69}$ Compliance to drugs was not assumed to be $100 \%$ in all studies. ${ }^{66-69}$

\section{Tuberculosis}

In $\mathrm{TB}$, key resistance issues are both primary resistance, where treatment-naïve patients can present with resistant TB strains, and secondary resistance, where resistance develops during treatment, leading to treatment failure and the need to switch to another regimen. The incidence of multi-drug resistance (MDR) is increasing in all TB populations, particularly those who are also HIV positive. Five economic evaluations in TB consider resistance. In Brewer and colleagues, ${ }^{70}$ the impact of different levels of MDR TB incidence was tested in sensitivity analysis, varied from 0.0001 to 0.139 , rates being taken from a published US survey. Threshold analysis (not completely reported) suggests that empirical MDR 
treatment is "cost-effective" where MDR TB rates exceed $9.6 \%$. The probability of drug treatment ADRs was incorporated into the model, along with attributable costs and mortality. Mortality associated with MDR TB was assumed to be the same as for standard strains of TB. Using empirical MDR treatment instead of waiting for culture results increased mortality by $2 \%$.

Directly observed therapy (DOT) is considered to reduce the emergence of MDR as improved compliance with the regimen prevents resistance developing within an individual patient during treatment. Weis and colleagues ${ }^{71}$ compared the costs and effects of DOT with standard therapy and reported a reduction in acquired resistance from 5.1 to $0.5 \%(p<0.001)$.

Suarez and colleagues ${ }^{73}$ compared three regimens with isoniazid monotherapy: (1) DOT MDR regimen for 18 months; (2) DOT MDR plus individualised treatment for failures; and (3) DOT MDR plus new individualised treatment for failures based on MDR sensitivity. Resistance to regimens was handled by using published 'nonresponse' rates. The authors also examined the effect of impact of treatment on transmission, using the following parameters: reproduction number (number of secondary cases produced by primary case in the next generation), based on published sources; generation time (years), based on published sources; and duration of infectiousness in presence or absence of MDR regimens, based on published sources and authors' assumptions. ICERs (mean cost per DALY) were calculated excluding transmission benefits and including transmission benefits. ICERs were lower when transmission benefits were included.

Sterling and colleagues examined DOT versus DOT-plus in smear-positive pulmonary tuberculosis using a Markov decision analytic model with Monte Carlo simulation in a cohort over 10 years. ${ }^{74}$ They examined the effect of high $(10 \%)$ and intermediate $(3 \%)$ proportions of MDR, but assumed that resistance stayed the same over a period of 10 years. ICERs (cost per death averted) were higher where MDR resistance levels were lower.

Three studies report that delays caused by waiting for a positive screen increased mortality. ${ }^{70-72}$

There was no long-term consideration of transmission of resistance in four $\mathrm{TB}$
There was also no attempt to examine the potential effects of an increase in resistance over time in all studies. ${ }^{70-74}$

\section{MRSA resistance}

Two economic evaluations in MRSA consider primary resistance in terms of treatment failure. Sharpe and colleagues examined oral linezolid $(n=30)$ versus intravenous vancomycin $(n=30)$ in the treatment of MRSA-complicated, lower extremity skin and soft-tissue infections in adults. ${ }^{75}$ VandenBergh and colleagues examined mupirocin nasal ointment $(n=868)$ versus nothing $(n=928)$ in cardiothoracic surgery. ${ }^{43}$ There was no long-term consideration of transmission of resistance in either study.

\section{Summary}

In summary, the lessons learnt from this review are:

- Resistance levels: Most studies considered resistance as a component of treatment failure. Most studies only used estimates of initial levels of resistance rather than observed figures, only two TB studies used observed resistance rates. ${ }^{70,71}$ Published non-response rates were used as an empirical measure of resistance in one study. $^{73}$

- Transmission of resistance: Transmission effects were only examined in one study in TB, using reproduction numbers based on published sources, generation time (years) based on published sources and duration of infectiousness. $^{73}$

- Changes in resistance over time: Increases in resistance over time were examined in two malaria studies only, and these increases were author estimates, not observed increases. ${ }^{68,69}$

- Impact of intervention on resistance: Only one study measured the effect of the intervention on resistance. $^{71}$

- Decision-makers' time preference: This affects the impact of resistance on the choice of optimal intervention.

For any particular microorganism, little is known about current levels of resistance, the rate of development of resistance transmission rates and the impact of interventions on resistance. The models presented in these evaluations are indicative only. They also take a relatively narrow perspective. Despite the longitudinal nature of some of these models, there is no incorporation of evidence regarding the societal impact caused by the adverse health effects of resistance. 


\section{Use of epidemiological and decision analytic techniques to model antibiotic resistance}

\section{Introduction}

A selective review was undertaken based on published papers which provided examples of epidemiological and decision analytic techniques to model antibiotic resistance. The aim was to learn from innovative methodological approaches to the complex issue of modelling resistance. Ideally, modelling of resistance would allow us to obtain quantitative estimates of the change in the rate of resistance, and subsequent costs and consequences, arising from a decision to use glycopeptides prophylactically.

\section{Antibacterial resistance}

Antibiotic resistance arises due to selection. Genetic mutations that confer resistance to antibiotics are selected for in the presence of antibiotics as antibiotic-sensitive organisms are removed from the population and antibioticresistant bacteria thrive. However, resistance to a particular antibiotic can develop in the absence of that antibiotic, due to the transmission of antibiotic-resistant bacteria between individuals, due to the existence of common modes of resistance across antibiotics and also due to the exchange of genetic material (plasmids and transposons) between bacteria. The increased incidence of antibiotic-resistant infections has led to increased morbidity and mortality associated with infection and is especially worrying in the light of the likely lack of new antibiotics in the near future. ${ }^{76,77}$

\section{Effect of prophylactic usage on the development of resistance}

Although the widespread use of antibiotics, including their prophylactic use, has been identified as one factor leading to an increase in antibiotic resistance, it has been suggested that it is not axiomatic that the prophylactic use of antibiotics increases the total usage of antibiotics and rate at which resistance develops. In the Effective Healthcare Bulletin

(http:/www.york.ac.uk/inst/crd/ehc45.htm) entitled 'Antimicrobial prophylaxis in colorectal surgery', it was suggested that the "appropriate use of antimicrobial prophylaxis in colorectal surgery may help to reduce the development of antibiotic resistant bacteria" as "by preventing postoperative wound infection, single dose or short-term antibiotic prophylaxis can reduce the need for long-term antibiotic therapy and therefore may contribute to reducing selection of antibiotic resistant bacteria". In a similar vein, the SIGN guideline entitled 'Antibiotic prophylaxis in surgery'3 suggests as a possible cost-effectiveness decision rule that prophylaxis should be given if "it is likely to reduce overall antibiotic consumption the hospital" and goes on to suggest that prophylaxis is highly recommended if prophylaxis unequivocally reduces major morbidity, reduces hospital costs and [authors' emphasis] is likely to decrease overall consumption of antibiotics. However, it was suggested by consultees during the course of this project that the overall consumption of antibiotics was not a good proxy for risk of the development of resistance. Although the rate at which antibiotic resistance develops will increase as the usage and so the rate of exposure of bacteria to antibiotic increases, it will also be affected by therapeutic practice and patient characteristics, the presence of a large bacterial innoculum as a reservoir of resistant mutants and the use of insufficiently high drug doses may increase the rate of resistance. ${ }^{77,78}$

Specifically, it was suggested by the consultees that resistance may be more likely to develop where short prophylactic courses are used compared with longer therapeutic courses, even where the overall antibiotic usage is similar.

\section{Results}

The models of antibiotic resistance identified during this review could be grouped (with examples) as follows.

\section{Models of the development of resistance in an experimental system}

De Gelder and colleagues developed mathematical models to explain the loss of resistance observed within culture bacteria. ${ }^{79}$

\section{Models predicting resistance rates in localised clinical environments}

Lipstitch and Bergstrom ${ }^{80}$ and Sebille and colleagues $^{81}$ produced models of the rates of antibiotic resistance within an intensive care unit (ICU) to investigate the impact of local control measures. The model was used to investigate the effect of various factors, such as transmission rates within the hospital, length of stay, antimicrobial policy and handwashing policies use on the persistence of resistance in the unit. These models focused on the rates observed within the unit and did not predict changes in the rates observed within the wider community resulting from changes in practice. 


\section{Models treating the rate of resistance as an exogenous variable}

Khan and colleagues ${ }^{82}$ developed a decision model of the management of latent tuberculosis infection which identified the optimum intervention for a range of geographical regions conditional upon the rate of drug resistance observed in each region. Goodman and colleagues ${ }^{68}$ developed a decision model to identify the optimum first line drug as a function of time for malaria treatment conditional on different assumptions regarding the development of drug resistance over time. The model was conditional on assumptions regarding the relationship between the decision to adopt a therapy and the subsequent rate of development of resistance to that therapy. Although these models did address the impact of changes in resistance between geographic regions or over time, they treated the rate of resistance essentially as exogenous variables and did not directly model the relationship between the therapeutic decisions and the subsequent rates of development of resistance.

\section{Empirical studies of the rate of development of resistance}

Mahamat and colleagues ${ }^{83}$ and Lopez-Lozano and colleagues $^{84}$ used ARIMA time series models to examine the empirical relationship between the uses of fluoroquinolone and resistance amongst Escherichia coli urinary tract isolates and between the use of both ceftazidime and Gram-negative bacilli and imipenem and Pseudomonas aeruginosa, respectively.

\section{Summary}

No models were identified which would prospectively predict the change in rate of development of resistance in the global clinical population arising from a change in therapeutic practice. This is perhaps not surprising considering the complexity of and uncertainty surrounding potential interactions between geographic and therapeutic patient populations, the mechanism and dynamics of the development of antibiotic resistance and the potential for crossresistance and the potential interactions between bacterial populations arising from the exchange of genetic material (Figure 14).

Predictive models of resistance in a local population have been developed and more global decision models commonly condition on estimates of the rate of resistance. Empirical models are available to evaluate retrospectively the impact of a change in therapeutic practice on the development of resistance.

\section{A conceptual framework for the economic evaluation of policies against MRSA}

\section{Introduction}

Although this report focuses on a very specific question - the cost-effectiveness of glycopeptides as prophylaxis against MRSA in high-risk surgical patients - the issue of drug resistance potentially broadens the evaluative questions markedly. This section considers the complexities associated with evaluating policy options for controlling MRSA (indeed, managing any disease where resistance is an issue). Informed by this, it also describes what an ideal evaluative framework might be for these sorts of policy options. Although the costeffectiveness analysis reported above is based on a very specific part of this framework, it is important to recognise the other elements of a full evaluation that are not explicitly considered in this 'partial' analysis. Although not a formal systematic review, this section is informed by a formal literature search using the strategy defined in Appendix 1.

The next section considers different dimensions of the evaluation problem regarding MRSA and, for each, what method might be used to deal with these complexities. A third section assesses the basis on which simplifications might be made to an evaluation such that it might be tractable analytically but of value to guide decision-making.

\section{Dimensions of complexity in evaluating policies for MRSA control The interdependence of decisions}

In most areas of health technology assessment, it is possible to isolate the decision problem being addressed from others. For example, in considering the cost-effectiveness of a new cholesterol-lowering therapy for primary prevention of heart disease, the use of the therapy is unlikely to influence the effectiveness or cost of other interventions. This is still a simplification in that the cost-effectiveness of an intervention which extends life sufficiently for enough patients to receive the new cholesterol-lowering therapy may hinge on the latter's cost-effectiveness. In other words, the new cholesterol-lowering therapy may extend an individual's life sufficiently to make saving their life with an apparently independent intervention cost-effective. However, these may be considered weak interdependencies which can safely be assumed away because policy decisions are unlikely to be sensitive to whether or not they are included. 


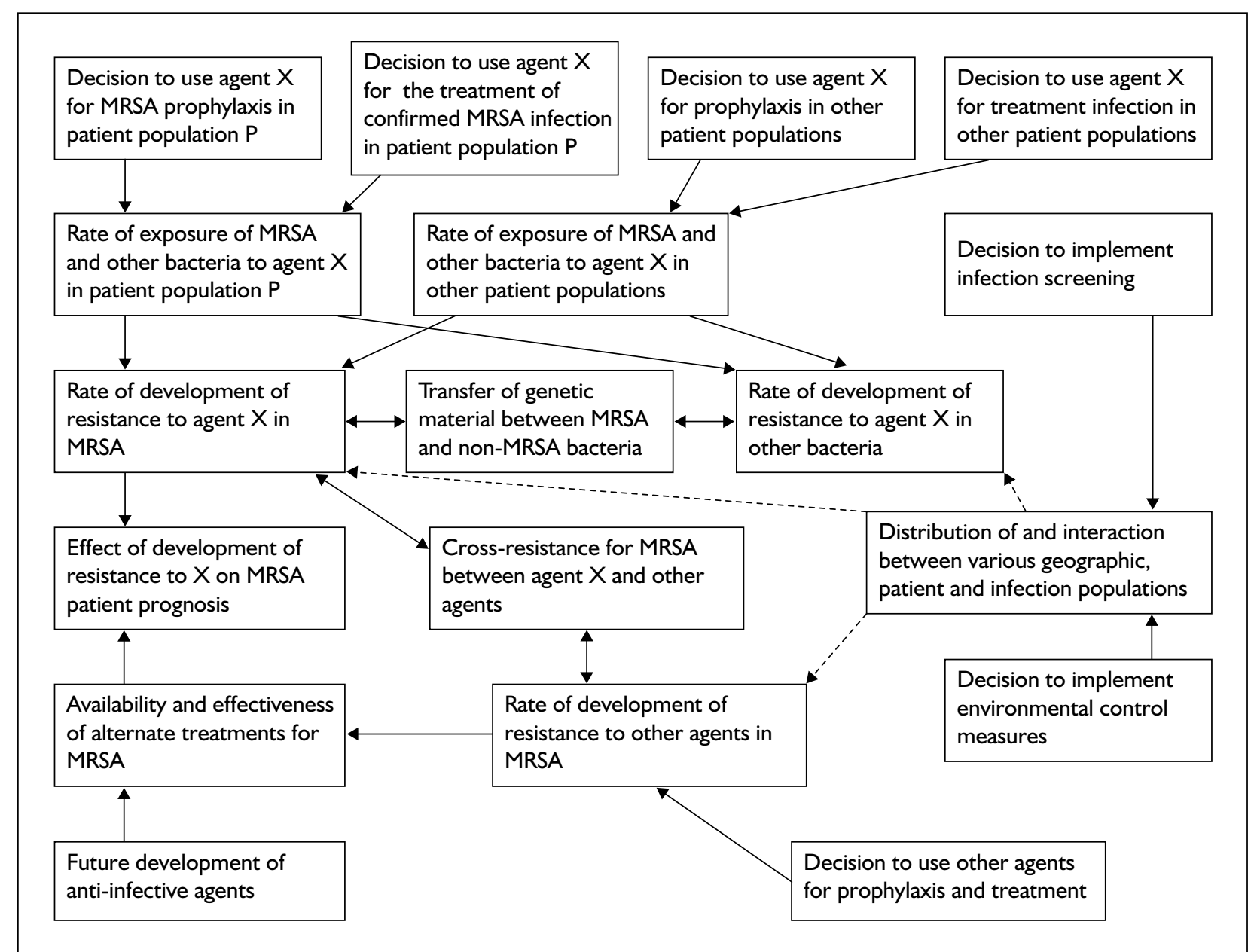

FIGURE I4 Potential interactions in a global model of the development of antimicrobial resistance consequences

In the case of managing diseases with therapies where resistance is an issue, the assumption of interdependence is stronger. This is illustrated in Figure 14, which shows the relationship between different types of decision and clinical effects in the context of the use of a given antibiotic. The interrelated decisions include whether to use the antibiotic as a prophylactic in one specific patient population, whether to use it as a prophylactic in one or more other patient populations, whether to use it as a treatment (of clinical infections) in a specific patient population, whether to use it as a treatment in one or more other patient populations and whether to use other MRSA control strategies (e.g. MRSA screening or environmental controls).

Typically, the decisions (at least to the extent to which they apply to different patient populations) would be considered independent. However, Figure 14 shows that this assumption may be unsafe to the extent that the decisions interact through the rate of exposure (in different patient populations) of MRSA to one or more agents and the rate of development of MRSA resistance to one or more agents. In short, the cost-effectiveness of a range of policy options available for one patient group (e.g. whether to give a particular antibiotic to patients undergoing hip replacement in one hospital) may influence the costeffectiveness of policy options for another patient group [e.g. whether to use that antibiotic (or, because of cross-resistance, another) in patients having heart surgery in another hospital].

How can an evaluative framework be developed to reflect this interdependence? In principle, this can be dealt with as an extension to standard decision modelling by linking the range of decisions. Thus such a model would, in effect, be simultaneously informing a cluster of decisions - that is, because of interdependence, it would make little sense to make decisions one at a time, and they should be addressed at the same time. Such decision models would make use of the epidemiological models that have been developed to model resistance (see 
the section 'Use of epidemiological and decision analytic techniques to model antibiotic resistance', p. 55), but also include appropriate parameters for economic analysis such as cost, utilities and prognosis. Although, given time and funding, such models could be developed, they would have to overcome two important challenges. The first is the difficulty of forecasting how drug resistance will develop over time, given its variability and the limited evidence base. This is a specific example of a general modelling problem of handling (often extreme) uncertainty in decision models in terms of parameter estimates. Although there is no 'magic bullet' to overcoming uncertainty, recent methods developments have provided a framework to quantify uncertainty in parameters ${ }^{85}$ express this in terms of decision uncertainty ${ }^{86}$ and assess the implications for research priorities and design. ${ }^{87,88}$

The second challenge is the need to decide where the boundaries would be drawn. That is, which decisions would formally be included in the model given that, in theory, there are numerous interdependencies. As for the example of the cholesterol-lowering therapy in the "standard economic analysis' described above, a judgement would have to be taken as to when the strength of the interdependency is sufficiently weak to leave out of the model under the assumption that its inclusion is unlikely to change the results.

\section{Large range of possible policy options}

In the face of antimicrobial drug resistance, there is a large range of possible policy options. This was emphasised by Coast and Smith, who identified 27 groups of policy options to contain antimicrobial resistance. ${ }^{89}$ These are summarised in Table 13. In principle, these policy options could be used individually or in combination. In terms of economic evaluation, they could be considered to be relevant comparators (individually or in combination) to each other and should be assessed in the same model. This would pose a number of challenges. As for modelling resistance, the first would be the uncertainty in the input parameters. In particular, there is likely to be limited and poor quality trial data comparing the different options. As described earlier, decision theory and value of information methods provide a potential way through this. A second, and related, problem is the lack of head-to-head trial data comparing all the policy options. This is not unusual in many technology assessment activities, and methods of indirect and mixed treatment comparisons exist to synthesise data in the absence of head-to-head trials. ${ }^{90}$
Despite the availability of these methods, however, the simultaneous comparison of all these policy options remains a major task. Some degree of prioritisation would seem appropriate - for example, some of the policy options may simply not be relevant for some local health systems. It would, of course, be necessary for this work to be adequately resourced and given a realistic timetable.

\section{Infectious disease}

The problem of resistance is a major source of added complexity in considering appropriate patient management regarding MRSA. However, the evaluation of policy options in the context of infection which can spread from one individual to another represents another level of complexity, and this clearly applies to MRSA, although the spread of the infection is typically in its preclinical stage. The implication of infection is that it is not necessarily reliable to assume that the rate of infection amongst a susceptible population is fixed as would typically be the case in decision models. In reality, the rate of infection is a function of the number of infected individuals in the community and should also include individual level characteristics (e.g. whether or not the individual is immune-compromised) and context level characteristics (e.g. the rate of infection within the hospital or the unit where the individual is located). Alongside this, consideration of the risk of carriage and the uncertainty of the relationship between carriage and infection is relevant. This increases the challenges associated with capturing the consequences of a change in the resistance rate. However, it is possible to adapt decision models to make them 'dynamic'. That is, they explicitly allow for the effects of herd immunity in a way that cannot be achieved with a static model ${ }^{91}$ Such models have been used for the economic evaluation of interventions to control infectious diseases, such as vaccination [which is an option in the context of MRSA (see Table 13)]. ${ }^{92}$ A key issue to consider is the extent to which failing to reflect the dynamic nature of a disease process in a decision model will lead to potentially misleading results and policy guidance.

\section{Cross-sectoral and macro economic effects}

Another feature of the evaluation of MRSA control activities is the fact that the costs and effects of alternative options will often extend outside the healthcare sector. This 'inter-sectoral' impact is a feature of the evaluation of many public health programmes and interventions. For example, in evaluating interventions for individuals with illegal drug dependence, there are likely to be effects 
TABLE I3 Policy strategies for containing antimicrobial resistance $^{89}$

\author{
Strategies for containing the emergence of \\ resistance

\section{By optimal use} \\ Antimicrobial cycling \\ Removal of potential septic foci/prostheses \\ Choosing the optimal agent, dose and dosage frequency \\ Use of drug combinations \\ By reducing use through the use of alternative \\ treatments \\ Use of antiseptics \\ Use of cranberry juice for urinary tract infection \\ Using probiotics \\ By reducing use through provision of improved \\ immunity \\ Increased vaccination \\ Improving nutrition \\ Minimise time patient immunocompromised \\ By reducing use without providing an alternative \\ treatment \\ Education of professionals \\ Patient education \\ Rapid diagnosis \\ Control of sensitivity data related to prescribers \\ Antimicrobial policies \\ Restriction of drug availability \\ Regulation of the use of antimicrobials in agriculture \\ Financial incentives
}

\section{Strategies for containing the transmission of resistance}

By early recognition of resistance

More rapid diagnostic techniques

Screening of patients/staff

Surveillance

By reducing infectivity

Use of antimicrobials to reduce infectivity

By reducing transmission possibilities

Isolation

Handwashing

Improvements in bed spacing

By reducing susceptibility to infection

Improve immunity by vaccination to reduce susceptibility Improve nutrition

(resource and other) on other public sector activities, most notably criminal justice. In the case of MRSA infection (and antibiotic resistance more generally), there are potentially extensive macroeconomic effects. ${ }^{93}$ At a domestic level, this would involve the adverse health effects of infection and resistance (from MRSA, but also susceptibility to other infections) leading to reduced productivity at work, which, other things being equal, would reduce national output and income. In turn, this would lead to reduced national savings, and hence investment, as well as welfare. In terms of production, lower productivity can lead to a reduction in company profits and hence in investment and employment. Increasing unemployment, together with lower profits and output, can reduce government revenues and increase government transfers; this can lead to reductions in health expenditure. Overall, there will be a reduction in societal welfare that, in principle, can extend much further than just health. At the broadest level, the problems of infection and resistance can have international implications in that, through individuals travelling abroad, drug resistance can spread. ${ }^{94}$

Although much depends on the extent of MRSA and other forms of antimicrobial resistance, the standard economic evaluation may be very limited in informing decisions about policy options. This is because these studies typically focus on costs to the health sector and changes in health outcomes within a given jurisdiction. However, these macroeconomic effects, if they are strong enough, can have a wider set of resource and welfare effects beyond national boundaries. Furthermore, there is a dynamic effect of such 'health shocks': an initial health effect has knock-on effects across different sectors and over time. It should be said that these macro-economic effects are potentially important for a range of diseases - for example, the economy-wide effects of HIV in many African countries is well known, and there is concern about the effects of a pandemic in avian influenza.

Part of the reaction to these macro-effects is in terms of the policy options to be evaluated. At a domestic level, policy options may not be confined to the healthcare sector and could include wider industrial policies or government expenditure and taxation decisions. The international dimension in how the effects spread means that some degree of coordinated global policy response might be appropriate. ${ }^{89}$ How can evaluation methods be extended to reflect these wider effects?

There has been some consideration of the use of general equilibrium models for policy evaluation in this context. ${ }^{93}$ These are models, similar to those used by the Treasury and other economic forecasters, which explicitly quantify the economic knock-on effects of health shocks. For example, Smith and colleagues describe a "computable general equilibrium (CGE) model". It is based on an economy made up of a set of economic agents - consumers, producers and government. ${ }^{93}$ Each of these is assumed to have a maximand and to 
make various decisions to satisfy this maximand subject to constraints. For example, a consumer is assumed to maximise their utility by allocating their time to paid employment and using income to purchase commodities. The interaction of these economic agents - each maximising their maximand subject to the relevant constraints - can result in an equilibrium when a set of prices are reached at which the level of production and consumption within each sector results in the quantity of supplied goods equalling the quantity demanded across all sectors. The macro effects of resistance are modelled as an external shock to the system, and policy options assessed by how the shock affects the macro economy. By assessing the new (post-shock) equilibrium in each sector, it is possible to estimate the net costs and benefits of each policy. In Smith and colleagues' CGE model, changes in national income from alternative policies are estimated and the distribution of those changes across different groups, and also levels of production and employment. Measures of welfare (such as Hicksian compensating variation) can be used, within a cost-benefit analysis framework, to assess the efficiency of alternative policy options. Smith and colleagues' model estimates that MRSA of $40 \%$ in the UK would lead to a reduction in gross domestic product (GDP) of between 0.4 and $1.6 \%$ (£3-11 billion), or about $6-20 \%$ of NHS expenditures in $1995 .{ }^{93}$

\section{Modelling the future}

It can be seen that assessing the cost-effectiveness of programmes and interventions in the context of resistance involves modelling under considerable uncertainty. One aspect of this uncertainty relates to modelling the future. There are several aspects of this. The first relates to the fact that the potential for drug resistance can result in policies which improve the health of current patients actually having a negative health effect on future patients. In the context of prophylactic antibiotics in surgical patients for MRSA, one of the factors referred to previously about the interdependence of decisions is the fact that the use of an antibiotic (such as the glycopeptides) as a form of prophylaxis today may lead to an increase in resistance to that drug, which, in turn, may reduce the available treatments for patients with clinical infections in the future. In other words, today's health gains are at the expense of tomorrow's health decrements - this could be characterised as a form of an inter-generational distribution problem.

Standard economic evaluation methods can deal with the fact that some patients gain and some lose from a particular policy decision. This is achieved by simply estimating a net health effect (gainers minus losers) across patients. An example of this is the evaluation of therapies which are efficacious in some patients but have side-effects in others and it is not possible to distinguish these patients when the treatment decision is made. In such a situation, the health benefit of the intervention is an average of the health gain and the health reduction weighted by the proportions of patients who experience these two types of outcomes. However, this standard approach to deal with 'gainers' and 'losers' is used when these two types of individual are contemporaneous. The MRSA and resistance problem is that the gainers are likely to be today and the losers are likely to be future generations of patients needing an effective treatment for MRSA.

Although not standard in economic evaluation, this feature could be handled by aggregating costs and benefits across time. Instead of modelling the costs and benefits of a therapy in a cohort of patients today, the model could model an evolving cohort over time, which would include those future patients needing treatment for MRSA infection. This is analogous to some screening models where a prevalence cohort of individuals is augmented by incidence cases over times. It also has parallels with the dynamic infections models referred to in the previous section. An important methodological issue with such a model, however, relates to the role of discounting. This is conventional in economic evaluations to reflect positive time preference which is anticipated in individuals and society - that is, the desire to bring forward benefits and to delay disbenefits. ${ }^{34}$ The use of an evolving cohort in a model with conventional discounting would, therefore, reduce the estimated health decrements for future patients (requiring treatment) compared with the health benefits for current patients (requiring prophylaxis), which may be considered inappropriate.

A related issue concerns the fact that the decision about whether to use antibiotics such as glycopeptides for prophylaxis is characterised by 'irreversibilities'. That is, once the decision to use the drug in this way is made, on the basis of what is currently known, it may be impossible to reverse the decision. This is because the greater exposure of the population to the drug is likely to increase drug-resistant strains of the bacteria, and this is unlikely to be reversed. This situation contrasts with decisions about other therapies where, if more evidence emerges about the effects and costs of a therapy, a past decision can be reversed, albeit 
at some cost. The fact that a decision may be irreversible (or only reversible at high cost) has implications for how that decision is made. Methods have been suggested about how to reflect 'irreversibilities' in economic evaluation. These include option pricing methods which, using approaches developed in financial economics, seek to model the emergence of future evidence and estimate the costs and benefits of delaying decisions. ${ }^{95}$ These methods have not been used in applied economic evaluations in healthcare and require further work to make them appropriate to the type of decisions facing health system decision-makers.

Another feature of modelling the cost-effectiveness of management options related to MRSA is that the costs and benefits associated with current decisions are likely to rest crucially on the future development of health technologies. Perhaps the most obvious example is that the anticipated future costs and health disbenefits resulting from antibiotic resistance are likely to be less as new antibiotics are developed because resistance will not necessarily reduce treatment options over the longer term. However, reflecting these possible future developments in economic evaluations is difficult. Again, this is due to the marked uncertainty associated with forecasting the future.

\section{Conclusions}

This chapter has provided a review of literature relevant to the economic evaluation of glycopeptides in prophylaxis. A series of reviews have been undertaken that relate to applied studies of glycopeptides specifically, of other forms of prophylaxis, in MRSA and in areas where resistance is a problem. Reviews have also been presented of epidemiological modelling work relating to resistance, and conceptual issues in economic evaluation in which resistance is an issue. From these reviews, it is possible to draw several conclusions:

- Published economic evaluations of glycopeptides do not address the decision problem facing the NHS. None has been undertaken in the UK, no study compared the full range of clinical options and most studies used condition-specific measures of effect rather the generic measures of health which are essential for system-level decision making (e.g. QALYs).

- Across the various reviews, few insights were provided on how to assess cost-effectiveness in the context of resistance. No studies modelled cost-effectiveness alongside epidemiological models of resistance. Those studies that considered resistance at all in a quantitative manner did so using sensitivity analysis or as a component of treatment failure, and few studies used empirical estimates of resistance.

- In a number of important respects, the economic evaluation of interventions in the areas where drug resistance is an issue is characterised by complexity. Although by no means unique compared to other disease areas, few others have the same number of complexities. In particular, the infectious nature of the disease, the interdependences of decisions and the extreme uncertainty about the future are major challenges for any evaluation.

- There are methods available which could begin to address these complexities. Some of these methods have been used before in economic evaluation - for example, the use of Bayesian statistical methods and value of information methods to be explicit about uncertainties and use these to prioritise future research. Other approaches have not been used very widely in the health field and would need further development - for example, macro-economic modelling. It is clear, however, that a major programme of research would be necessary to develop and apply these methods to the management of MRSA in the UK.

- In addition to estimating the cost-effectiveness of glycopeptide prophylaxis with regard to the costs and effects for the individual who may receive prophylaxis, it is also necessary to consider the potential for an increase in the rate of development of antibiotic resistance and its consequences across the future patient populations. Also, as it is likely that the effect of glycopeptide prophylaxis on antibiotic resistance will depend on the extent of usage, there is a need to consider the potential implementation across the patient population rather than simply considering the costeffectiveness for individuals. 



\section{Chapter 6 Economic model}

\section{Introduction}

In order to investigate whether there is a threshold level of MRSA prevalence at which it is cost-effective to switch from non-glycopeptide to glycopeptide antibiotic prophylaxis in surgical environments with a high risk of MRSA infection, the aim was to develop a new decision analytic model. There are a number of reasons why the modelling work presented in this chapter should be seen as indicative rather than definitive. The first of these is the number of major complexities associated with the economic evaluation of options in the field of MRSA (see the section 'A conceptual framework for the economic evaluation of policies against MRSA', p. 56). Second, the review of the economic evaluation evidence in Chapter 4 did not reveal any head-to-head studies which directly compared the prophylactic use of vancomycin and teicoplanin against all relevant non-glycopeptide prophylaxis comparators for use in surgery. No studies which evaluated glycopeptide prophylaxis were undertaken in the $\mathrm{UK}$, and infection control practices and SSIs are likely to differ substantially across settings. The supplementary literature reviews (see the sections 'Economic evaluations of antibiotics where antibiotic resistance is a problem', p. 46 and 'Use of epidemiological and decision analytic techniques to model antibiotic resistance', p. 55) provided few insights of how to assess cost-effectiveness of antibiotics in the context of resistance.

In addition, the results of the clinical review in Chapter 3 have highlighted the major limitations of the clinical evidence. In particular, only two studies reported the number of SSIs caused by MRSA. ${ }^{16,17}$ This dearth of data on the effectiveness of glycopeptides in the prophylaxis setting represents a major limitation in what is possible in terms of estimating their costeffectiveness.

It has therefore not been possible to reflect the range of complexities in MRSA modelling within the time available for the current analysis. Although methods are available that could begin to address these complexities, some of them have not been widely used in the health field and a major programme of research would be necessary to develop and apply these methods to the management of MRSA in the UK. Despite these difficulties, it is hoped that the indicative modelling presented in this chapter provides a contribution to decision making in this field. In particular, it describes a modelling approach that could be used more fully as stronger clinical data emerge. The model has been developed to estimate costs from the perspective of the UK NHS and Personal Social Services (PSS) and health outcomes in terms of QALYs and was developed in Excel.

\section{Exemplar: hip arthroplasty}

Based on the effectiveness and cost-effectiveness reviews, it is clear that resource use, costs and outcomes associated with antibiotic prophylaxis differ by type of surgery. Hence it was decided to focus on a single area of surgery, by way of an exemplar, for the indicative economic model. The surgical speciality chosen was hip arthroplasty for the following reasons:

1. Hip arthroplasty is classed as a 'clean' surgical procedure, that is, the operative wound is not likely to be infected or inflamed with preexisting infections and is closed. ${ }^{96}$

2. This group of patients is relatively homogeneous.

3. The group is at substantial risk of serious SSI including a high risk of MRSA infections.

4. Data availability is, in principle, greater in this type of surgery compared with others because of the high volume of orthopaedic surgery undertaken.

The use of surgical antibiotic prophylaxis is only recommended in procedures where there is a high risk of infection, such as colorectal surgery, or where the consequences of infection can be severe, such as joint arthroplasty. There is a general consensus that the minimum number and doses of antibiotics need to be administered in such a way as to provide sufficient cover during surgery, while at the same time prescribed appropriately to minimise the development of antimicrobialresistant bacteria. SSIs after hip arthroplasty result from a variety of factors, including bacterial contamination of the wound during the procedure, virulence of the contaminating organisms, factors 
within the surgical wound and patient risk factors such as concomitant disease. Use of prophylactic antibiotics is generally recommended after hip arthroplasty to minimise SSIs. ${ }^{97}$ A dose of an appropriate antibiotic is usually administered within 60 minutes of the surgical incision to ensure that adequate drug concentrations are present in the serum, tissue and wound during the entire time that the incision is open and at risk of bacterial contamination. Prophylactic

antimicrobials should then be discontinued within 24 hours after completion of the procedure.

\section{Methods}

\section{Purpose}

The purpose of the indicative modelling is to provide a framework within which the costeffectiveness of prophylactic interventions for high-risk surgery can be assessed. Using available evidence and clinically informed assumptions, the model seeks to illustrate how the threshold baseline risk of clinical MRSA infection at which the use of glycopeptides becomes cost-effective might be estimated. The analysis assessed the relative cost-effectiveness of vancomycin, cephalosporin or their combination.

Given the complexities and lack of data, as explained previously, a number of assumptions and simplifications are made in the analysis. Perhaps the most important of these is that longterm resistance to glycopeptides has not been explicitly modelled. It should be reiterated, therefore, that this analysis seeks to indicate how a model might be developed to estimate a threshold baseline risk; its results are of limited direct policy relevance.

\section{Model structure}

A decision model was developed to estimate the expected costs, the expected effects in terms of QALYs and the expected glycopeptide usage for patients receiving vancomycin, cephalosporin or vancomycin and cephalosporin used in combination. The decision model is illustrated in Figure 15. For each treatment arm the probability of no infection, MRSA infection and non-MRSA infection was estimated. For the two types of infection arms, the probability of a deep or superficial infection was estimated; this was assumed to be independent of whether it was an MRSA or non-MRSA infection. These rates were conditional on the 'baseline' MRSA infection rate with cephalosporin prophylaxis and non-MRSA infection rate with cephalosporin prophylaxis.
The odds ratios (ORs) for MRSA and non-MRSA infections for vancomycin prophylaxis compared with cephalosporin were estimated from a randomised trial. The baseline infection rates for cephalosporin prophylaxis were converted to odds. These were multiplied by the relevant ORs and converted back to probabilities to estimate the infection rates for vancomycin prophylaxis.

The baseline infection probabilities were converted into odds and multiplied by the relevant ORs for the prophylaxis options compared with cephalosporin prophylaxis.

For the no infection, deep and superficial infection populations, the risk of death was estimated; this was assumed to be independent of MRSA or non-MRSA infection. Costs were estimated for cephalosporin or vancomycin and cephalosporin prophylaxis and treatment for noninfected, deep and superficial MRSA and deep and superficial non-MRSA patients. QALYs were estimated for non-infected living patients and a utility decrement was incorporated for patients with deep and superficial infections. The utility decrement was estimated by multiplying the mean hospital stay by a utility decrement. In addition, the number of days of glycopeptide treatment for patients with deep and superficial MRSA infections was estimated.

\section{Parameters for use in the model}

The parameters in the model can be divided into the following categories:

- baseline infection rates: SSI rates; MRSA SSI rates

- effectiveness estimates for interventions in terms of the relative reduction in infection rates

- consequences of infection: impact on survival, length of hospital stay, health-related quality of life (HRQoL) and treatment intensity.

\section{Clinical effectiveness: which infection rates should be used?}

The model aims to identify the baseline MRSA SSI rate in a surgical patient cohort that would justify the use of prophylactic glycopeptide antibiotics, rather than non-glycopeptide antibiotics. As such, baseline surgical site MRSA infection rate is an output of the model rather than an input. However, to provide some context for these threshold estimates, it would be helpful to present some UK statistics on these risks. Nationally obtained infection control surveillance data report MRSA bacteraemia incidence rates in both medical and surgical hospital admissions. ${ }^{98}$ 


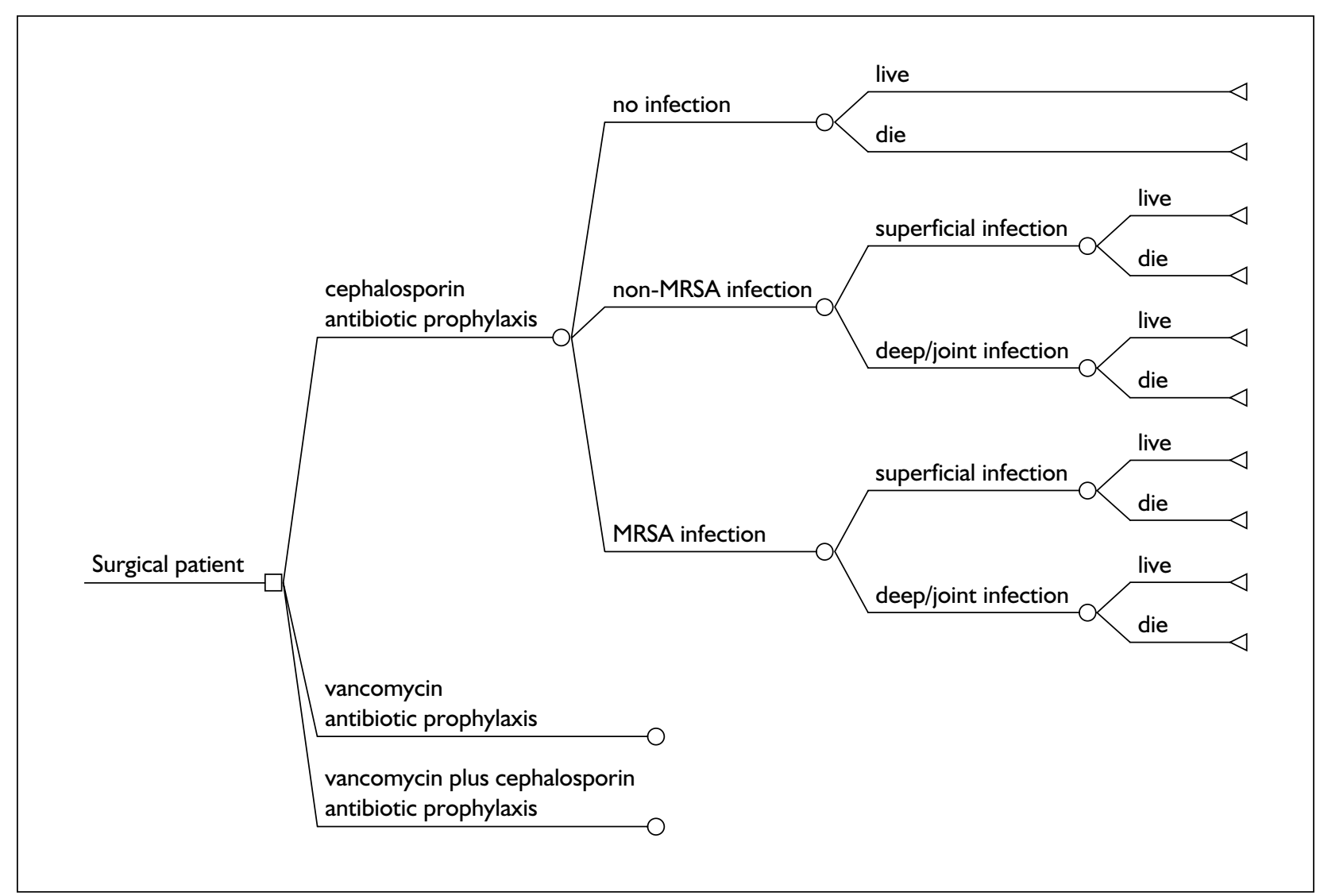

FIGURE I5 Model diagram

However, the link between bacteraemia rates and SSI rates is not well understood and, therefore, it is not appropriate to apply the infection control surveillance data that use MRSA bacteraemia incidence rates within our model.

The Surgical Site Infection Surveillance Service (SSISS), formerly known as the National Nosocomial Infection Surveillance Service (NNIS), was established by the Department of Health and the Health Protection Agency in $1996 .{ }^{99}$ Using CDC definitions for infections (Table 14), this service provides information from 102 hospitals in England, reported by surgical procedure, and provides information on causative organism, severity of infection and length of hospital stay. ${ }^{100}$ The results from this study reflect considerable variation between hospital SSI rates and the need for individual hospitals to be aware of their local SSI rates for application to our model. These data would make the model's estimate of a threshold relevant to local decision-makers because individual hospitals have information on their SSI rates. Therefore, they will be able to determine whether their MRSA infection risk is above or below thresholds derived in the model at which glycopeptides are potentially cost-effective.
SSISS figures from a survey of 102 English hospitals suggested that 363/16291 (2.23\%, 95\% CI 2.0 to $2.5 \%$ ) primary total hip arthroplasties resulted in SSI. Of these, $294(1.80 \%)$ were superficial wound infections, $38(0.23 \%)$ were deep wound infections and $30(0.18 \%)$ were joint infections. ${ }^{99}$ MRSA appears to be the most common pathogen to cause early SSI in hip arthroplasty; ${ }^{99} 24.3 \%$ were positive for MRSA, $21.9 \%$ for MSSA and $\mathbf{1 5 . 3 \%}$ for CNS.

Coagulase-negative Staphylococcus sp. may be more important in deep infections involving the joint that ultimately lead to late joint failure. MRSA may play a limited role in late hip arthroplasty failure. ${ }^{101}$ Therefore, the use of glycopeptide or non-glycopeptide antibiotics around surgery is not likely to have an effect on late infective failure. As a result, the primary analysis reported here examines the impact of a change in antibiotic policy on the incidence of early SSIs only.

\section{Baseline MRSA SSI rates}

In our model, the baseline incidence of SSI due to MRSA was $1.2 \%$ in the base case, with a plausible range of $0-0.3 \%$ from Zanetti and colleagues; however, this related to CABG, not orthopaedic 
TABLE I4 Infection definitions used in the SSISS database 100

\begin{tabular}{|c|c|}
\hline Type of infection & Definitions \\
\hline Superficial incisional & $\begin{array}{l}\text { Occurs within } 30 \text { days of surgery. } \\
\text { Involves only skin and subcutaneous tissue and meets at least one of the following criteria: } \\
\text { I. Purulent drainage from superficial incision. } \\
\text { 2. Organisms are grown and pus cells seen from aseptically obtained swab/tissue from the } \\
\text { superficial incision. } \\
\text { 3. At least two of the following signs and symptoms: } \\
\text { (a) pain or tenderness, localised swelling, redness or heat, and } \\
\text { (b) (i) the clinician diagnoses an infection or (ii) the superficial incision is deliberately opened } \\
\text { by a surgeon to manage the infection, unless the incision is culture-negative. }\end{array}$ \\
\hline Deep incisional & $\begin{array}{l}\text { Occurs within } 30 \text { days (no implant) or I year (implant) of surgery. } \\
\text { Involves deep fascia and muscle layers. } \\
\text { Appears to be related to the procedure and meets at least one of the following criteria: } \\
\text { I. Purulent drainage from the deep tissue but not the joint or bone. } \\
\text { 2. Organisms are grown and pus cells seen from aseptically obtained swab/tissue from the deep } \\
\text { incision. } \\
\text { 3. A deep incision which spontaneously dehisces or is opened by the surgeon when the patient } \\
\text { has fever }\left(>38^{\circ} \mathrm{C}\right) \text {, localised pain or tenderness unless the incision is culture-negative. } \\
\text { 4. An abscess or other evidence of deep infection found during re-operation, or by } \\
\text { histopathological or radiological examination. }\end{array}$ \\
\hline Joint/bone infection & $\begin{array}{l}\text { Occurs within } 30 \text { days (no implant) or I year (implant) of surgery. } \\
\text { Involves joint and/or bone related to the site of the operation with any other tissues. } \\
\text { Appears to be related to the procedure and meets at least one of the following criteria: } \\
\text { I. Purulent drainage from a drain which is placed through a stab incision into the joint. } \\
\text { 2. Organisms are grown and pus cells seen from aseptically obtained swab/tissue from the } \\
\text { joint/bone. } \\
\text { 3. An abscess or other evidence of joint/bone infection found during re-operation, or by } \\
\text { histopathological or radiological examination. } \\
\text { 4. The patient has at least two of the following signs or symptoms with no other recognised } \\
\text { cause: joint pain, swelling, tenderness, heat, evidence of effusion or limitation of movement } \\
\text { and at least one of the following: } \\
\text { (a) organisms and white blood cells seen on Gram stain of the joint } \\
\text { (b) positive antigen test on blood, urine or joint fluid } \\
\text { (c) cellular profile and chemistry of joint fluid compatible with infection and not explained by } \\
\text { an underlying rheumatological disorder } \\
\text { (d) radiological evidence of infection, e.g. abnormal findings on radiographs, CT scans, MRI, } \\
\text { radiolabelled scan (gallium, technetium, etc.). }\end{array}$ \\
\hline
\end{tabular}

surgery. ${ }^{37}$ The purpose of the model was to identify the threshold value, within this range, at which glycopeptides might be considered costeffective.

\section{Treatment effect}

The treatment effect of vancomycin was applied to this baseline rate. This assumes that standard practice in the UK is to use a non-glycopeptide for antibiotic prophylaxis in primary hip arthroplasty, as currently recommended by the British Orthopaedic Association. ${ }^{97}$

The effectiveness review reported in Chapter 3 shows how few data are available on the effect of glycopeptides specifically on MRSA SSIs, and that no orthopaedic surgical trials reported MRSA rates. The treatment effect used was, therefore, derived from the wider set of effectiveness data from other clean surgery trials to this baseline MRSA rate.

Of the SSIs that occur after primary hip arthroplasty, $80.7 \%$ are superficial wound infections, $10.3 \%$ are deep wound infections and $8.1 \%$ are joint infections. ${ }^{2}$ In the model, it is assumed that the three categories of SSIs occur with this relative frequency in all treatment arms. It was assumed that the treatment effect of glycopeptides was equal across all categories of SSIs. Finally, it is assumed that $24.3 \%$ SSIs are MRSA, equally across all categories of SSIs. ${ }^{99}$ 


\section{Consequences of infection}

The consequences of infection can be divided into

(1) impact on survival, (2) length of hospital stay,

(3) HRQoL and (4) treatment intensity.

\section{Mortality attributable to infection}

From the trials included in the effectiveness review, it was not possible to determine mortality attributable to infection as these studies were small to estimate the risk of such a rare event.

Data on mortality attributable to SSI in general, based on Ridgeway and colleagues' SSISS study, ${ }^{99}$ were analysed, with mortality for hip arthroplasty patients with SSIs being adjusted for confounders [age, sex, American Society of Anesthesiology (ASA) class, wound class, elective/emergency surgery, duration of operation, complexity of operation, trauma, time in hospital to operation]. ${ }^{2}$ Mortality without an SSI was $2.4 \%$, versus $7.4 \%$ with SSI (adjusted OR 1.8, 95\% CI 1.3 to 2.7; $p<0.002$ ). Mortality for superficial SSI was $5.7 \%$, and for deep/joint SSI it was $11.9 \%$.

Mortality attributable to MRSA SSI was not reported in Coello and colleagues' SSISS study. ${ }^{2,99}$ MRSA infection does not appear to lead consistently to increased mortality over other types of infection. The underlying comorbidity of patients who develop MRSA infections appears to confound and exaggerate the effect of MRSA. ${ }^{102}$ In our model, it is assumed that MRSA infections have the same attributable mortality as other infections.

\section{Length of hospital stay attributable to infection}

SSI is likely to increase length of hospital stay (LOS), due to the interval from detection, through treatment and infection resolution to discharge. From the trials included in the effectiveness review, it was not possible to determine LOS attributable to infection.

From SSISS data, the median time to detection of SSI was 8 days for superficial incisional, 11 days for deep incisional and 11 days for joint/bone infections. For each procedure, the mean length of stay doubled for patients with SSI. ${ }^{99}$ The median LOS was 9 days for primary total hip arthroplasty patients without SSI and 17 days for patients with SSI. These LOSs have not been corrected for confounding factors and means (necessary for the decision model) were not reported. However, these data were analysed further and increased LOSs for hip arthroplasty patients with SSIs were adjusted for confounders (i.e. age, sex, ASA class, wound class, elective/emergency, duration of operation, complexity of operation, trauma, time in hospital to operation). ${ }^{2}$ The geometric mean LOS for hip arthroplasty patients without SSI was 11.1 days. The mean extra LOS attributable to all SSIs, identified though multivariate analysis, was 11.5 days (95\% CI 10.3 to 12.8 days). The mean extra LOS attributable to superficial SSI was 8.9 days (95\% CI 7.7 to 10.2 days). The mean extra LOS attributable to deep/joint SSI was 22.8 days (95\% CI 19.2 to 26.9 days).

The presence or absence of an increased LOS due to MRSA over other infections was not reported in the SSISS studies. ${ }^{2,99}$ Increased LOS has been reported for MRSA bacteraemia compared with MSSA (9 versus 7 days, $p=0.045$ ). ${ }^{103}$ In this study, after correcting for confounders, the authors report a median attributable length of stay of 2 days. It is not clear whether these data, from a 1997-2000 US cohort of bacteraemia patients, are applicable to hip arthroplasty-associated SSI. In a model of the cost-effectiveness of different MRSA screening methods, Kunori and colleagues added a range of number of days LOS to account for MRSA screening delays. ${ }^{104}$ However, when treating an SSI, cultures would be taken whether the infection was MRSA or not, so there would be no incremental difference. In the absence of compelling evidence to the contrary, it is assumed that there is no increased LOS due to MRSA over other infections.

\section{Quality of life, utility data and QALYs}

As noted in the review of economic evaluations of glycopeptides, only one study included a generic measure of health, based on QALYs. ${ }^{37}$ However, it was not possible to use these QoL weights since they were direct valuations of health states specific to cardiothoracic surgery. One study did use a generic outcome measure [Short Form with 36 Items (SF-36)] in orthopaedic surgery in order to assess the impact of SSIs 1 year after their initial detection, post-orthopaedic surgery. ${ }^{105}$ The study was based on a pairwise, matched control within cohort study. All patients had undergone orthopaedic surgery and the case patients had an SSI postoperatively whereas the control patients were free of SSIs. It was found that case patients experienced substantial reductions in their HRQoL 1 year post-SSI detection compared with the control patients, with the largest decrements in their HRQoL observed in the physical functioning and role-physical domains. It is possible to generate utility scores based on the SF-36 scores; however, to do so would have required access to individual patient data and the authors of the study did not respond to requests for access to these data. 
Tengs and Wallace have identified $1000 \mathrm{HRQoL}$ estimates based on a review of the literature and found a utility weight for an infection relating to an artificial joint of $0.9 .{ }^{106}$ Therefore, infection was related to a 0.1 decrement in utility. In order to convert these data into QALYs, four elements were combined:

1. A utility decrement associated with infection of 0.1 was calculated, based on Tengs and Wallace's data. ${ }^{106}$

2. The mortality associated with infection (as described in the section 'Mortality attributable to infection', p. 67) from which the probability of dying with no infection, a superficial infection and a deep/joint infection were calculated. ${ }^{2}$

3. Life expectancy [based on life-tables for 65-year-old men in the UK based on data for 2002-2004 (http://www.gad.gov.uk/Life_Tables/ Interim_life_tables.htm)].

4. The national QoL norms by age and sex (0.78 for 65-74-year-olds and 0.73 for $\geqslant 75$-year-olds) (http://www.york.ac.uk/inst/che/ pdf/DP172.pdf).

QALYs (and costs) were discounted at 3.5\% per year. Patients who survived an infection experienced 8.74 discounted QALYs over a lifetime (based on males aged 65 years).

\section{Infection-related interventions and treatment intensity}

Of the SSIs that occur after primary hip

arthroplasty, most are superficial wound infections, with only $18.4 \%$ being deep wound or joint infections. ${ }^{99}$ Superficial infections are likely to require less intensive intervention than deep or joint infections. Interventions following infection can be minor, such as antibiotics, or there can be a more major requirement for surgical intervention, either for debridement of the wound or for revision arthroplasty. ${ }^{107}$ In a study of a cohort in Avon, early infection after primary hip arthroplasty occurred in 14 out of 1567 procedures, and eight were treated with antibiotics, two had exploration, debridement and washout and four had exploration, debridement and washout, and a revision procedure. ${ }^{107}$

Based on this study and clinical advice, it is assumed that the following level of intervention occurs for each severity of SSI:

- superficial SSI: antibiotic treatment

- deep/joint SSI: exploration, debridement and washout, antibiotic treatment.

There are a number of antibiotics used for treatment of non-MRSA infections. For the purpose of this particular exemplar, we have assumed treatment with oral erythromycin (Kay P, Consultant Orthopaedic Surgeon: personal communication, 2006).

\section{MRSA infection-related interventions and treatment intensity}

Superficial MRSA infections are treated with intravenous vancomycin. Deep or joint MRSA infections are treated with intravenous vancomycin and oral rifampicin ${ }^{4}$ (Kay $\mathrm{P}$, Consultant Orthopaedic Surgeon: personal communication, 2006).

Patients who develop an MRSA infection will require isolation and barrier nursing in addition to the interventions described above. Published UK estimates of the resource use associated with this suggest the following (Table 15). ${ }^{104}$

During an MRSA infection, patients would be barrier nursed in a side room, and will be discharged from hospital when they are MRSA negative. Table 16 summarises treatment pathways and mortality for non-MRSA and MRSA SSIs post hip arthroplasty.

\section{Results}

\section{Baseline infection rates}

In the model, the baseline MRSA SSI rates and non-MRSA rates with a cephalosporin were varied

TABLE I5 Isolation (barrier nursing) costs ${ }^{104}$

\begin{tabular}{|lll|}
\hline Cost component & Resource use & Cost $^{a}$ \\
\hline Barrier nursing costs per day & [gloves $(£ 0.02)+$ paper gown $(£ \mid .95)+$ & $£ 306.93$ per day (2002 prices); \\
& I minute labour $(£ 0.16)]$ multiplied by & inflated to 2005: $£ 348.27^{39}$ \\
& I44 room entries per day & \\
\hline${ }^{a}$ Source: Royal Free Hospital, London. & \\
\hline
\end{tabular}


TABLE 16 Treatment pathways and mortality attributable to infection

\begin{tabular}{|c|c|c|c|}
\hline Type of SSI & Treatment $^{107}$ & $\begin{array}{l}\text { Mean length of stay (days) } \\
(95 \% \mathrm{Cl})^{2}\end{array}$ & $\begin{array}{c}30 \text { day mortality } \\
(\%)^{2}\end{array}$ \\
\hline No SSI & None & 11.1 & 2.4 \\
\hline Superficial SSI & $\begin{array}{l}\text { Antibiotic treatment with: } \\
\text { Non-MRSA: erythromycin } \\
\text { MRSA: vancomycin }{ }^{4} \text { plus barrier nursing }\end{array}$ & 20.0 (18.8 to 21.3$)$ & 5.7 \\
\hline Deep or joint SSI & $\begin{array}{l}\text { Exploration, debridement and washout, } \\
\text { antibiotic treatment with: } \\
\text { Non-MRSA: erythromycin } \\
\text { MRSA: vancomycin and rifampicin }{ }^{4} \text { plus } \\
\text { barrier nursing }\end{array}$ & $33.9(30.3$ to 38.0$)$ & 11.9 \\
\hline
\end{tabular}

TABLE I7 Treatment effect ${ }^{17}$

\begin{tabular}{|llrrrrr|}
\hline Option & Event & $\boldsymbol{r}$ & $\mathbf{N}$ & $\mathbf{P}(\%)$ & Odds & OR \\
\hline Vancomycin & MRSA infection & 2 & 452 & 0.44 & 0.0044 & 0.2705 \\
& Non-MRSA infection & 41 & 452 & 9.07 & 0.0998 & 1.2501 \\
\multirow{3}{*}{ Cephalosporin } & MRSA infection & 7 & 433 & 1.62 & 0.0164 & \\
& Non-MRSA infection & 32 & 433 & 7.39 & 0.0798 & \\
\hline
\end{tabular}

from 0 to $1 \%$ and from 0 to $3 \%$, respectively. The baseline MRSA SSI rate was not a direct input to the model but was varied to find the threshold. To put this range in context, the mean overall SSI and MRSA SSI rates for hip arthroplasty in England are $2.23 \%^{2}$ and $0.54 \%$, respectively.

\section{Treatment effect of glycopeptides}

Only two studies in the systematic effectiveness review captured cases of MRSA (see Chapter 3). ${ }^{16,17}$ That by Kitzis and colleagues was a smaller study and only available in abstract form. ${ }^{16}$ Therefore, the treatment effect was obtained from Finkelstein and colleagues' study of vancomycin versus cefazolin prophylaxis for cardiac surgery in the setting of a high prevalence of methicillin-resistant staphylococcal infections, ${ }^{17}$ as summarised in Table 17.

Mortality data ${ }^{2}$ were combined with published utility weights ${ }^{106}$ and life expectancy estimates
(http://www.gad.gov.uk/Life_Tables/Interim_life tables.htm) to derive an estimate of effect of SSI on mortality and QALY production, as summarised in Table 18.

\section{Resource use and unit costs}

The resource use and costs associated with the following sections of the model were developed based on the following (see Tables 19 and 20):

- vancomycin prophylaxis

- cephalosporin prophylaxis

- inpatient hip arthroplasty episode

- management of superficial and deep/joint nonMRSA and MRSA infections.

\section{Incremental cost-effectiveness}

Table 21 summarises the effect of SSI, including type of SSI (superficial or deep/joint), on costs, QALYs and the average (mean) number of days' use of glycopeptides. Table 22

TABLE I8 Effect of SSI on mortality and QALYs

\begin{tabular}{|lclccc|}
\hline Event & QALY & Sub-event & Probability & QALY & Loss due to hospital stay \\
\hline No infection & 8.53 & Death & 0.024 & 0.00 & 0.0000 \\
& & Alive & 0.976 & 8.74 & \multirow{2}{*}{0.0030} \\
Superficial infection & \multirow{2}{*}{8.24} & Death & 0.057 & 0.00 & 0.0063 \\
& & Alive & 0.943 & 8.74 & 0.00 \\
Deep/joint infection & \multirow{2}{*}{7.70} & Death & 0.119 & 0.74 & 0.881 \\
\end{tabular}


TABLE 19 Treatment pathways and costs

\begin{tabular}{|c|c|c|c|}
\hline Resource use & Units & Cost $(t)$ & Source \\
\hline \multicolumn{4}{|c|}{ Treatment pathway and cost of vancomycin prophylaxis } \\
\hline $\begin{array}{l}\text { Vancomycin prophylaxis I g b.d. for } 24 \mathrm{~h} \\
\text { (BOA) }\end{array}$ & 2 & 32.22 & $\begin{array}{l}\text { http://www.boa.ac.uk/PDF\%20files/ } \\
\text { BOA\%20Hip\%20replacement.pdf }\end{array}$ \\
\hline $\begin{array}{l}\text { Administration costs: } 100 \mathrm{ml} \text { of } 0.9 \% \text { sodium } \\
\text { chloride solution }\end{array}$ & 2 & 0.60 & SPC (electronic Medicines Compendium) \\
\hline Total & & 32.82 & \\
\hline \multicolumn{4}{|c|}{ Treatment pathway and cost of cephalosporin prophylaxis } \\
\hline $\begin{array}{l}\text { Cefuroxime prophylaxis } 1.5 \mathrm{~g} \text { at induction, } \\
\text { followed by } 2 \text { doses of } 750 \mathrm{mg}(4 \text { ampoules } \\
750 \mathrm{mg} \text { ) (BOA/BNF) }\end{array}$ & 4 & 18.80 & $\begin{array}{l}\text { http://www.boa.ac.uk/PDF\%20files/ } \\
\text { BOA\%20Hip\%20replacement.pdf }\end{array}$ \\
\hline Administration costs: $10 \mathrm{ml}$ of water for injection & 3 & 0.93 & SPC (electronic Medicines Compendium) \\
\hline Total & & 19.73 & \\
\hline \multicolumn{4}{|c|}{ Treatment pathway and cost of a hip arthroplasty episode } \\
\hline Hip arthroplasty episode & I & 6060.60 & NHS reference costs; http://www.dh.gov.uk/ \\
\hline \multicolumn{4}{|c|}{ Treatment pathway and cost of superficial non-MRSA infection } \\
\hline $\begin{array}{l}\text { Non-MRSA antibiotic treatment with } \\
\text { erythromycin } 500 \mathrm{mg} \text { q.d.s. for } 7 \text { days }\end{array}$ & 28 & 5.32 & $\begin{array}{l}\text { Personal communication (P Kay, Consultant } \\
\text { Orthopaedic Surgeon) }\end{array}$ \\
\hline Administration costs: none & 0 & 0.00 & SPC (electronic Medicines Compendium) \\
\hline MRSA test & 1 & 7.09 & NHS reference costs; http://www.dh.gov.uk/ \\
\hline Inpatient day & 8.9 & 1780.00 & Coello, $2005^{2}$ \\
\hline Total & & $|792.4|$ & \\
\hline \multicolumn{4}{|c|}{ Treatment pathway and cost of deep/joint non-MRSA infection } \\
\hline $\begin{array}{l}\text { Non-MRSA antibiotic treatment with } \\
\text { erythromycin } 500 \mathrm{mg} \text { q.d.s. for } 14 \text { days }\end{array}$ & 56 & 10.64 & $\begin{array}{l}\text { Personal communication (P Kay, Consultant } \\
\text { Orthopaedic Surgeon) }\end{array}$ \\
\hline Administration costs:none & 0 & 0.00 & SPC (electronic Medicines Compendium) \\
\hline MRSA test & 1 & 7.09 & NHS reference costs; http://www.dh.gov.uk/ \\
\hline Inpatient day & 22.8 & 4560.00 & Coello, $2005^{2}$ \\
\hline Wound exploration & 1 & 1107.00 & Blom, $2004^{108}$ \\
\hline Total & & 5684.73 & \\
\hline
\end{tabular}

TABLE 20 Unit costs used in the model

\begin{tabular}{|c|c|c|}
\hline Item & Unit cost ( $(€)$ & Source \\
\hline Vancomycin I g injection & 16.11 & BNF 50 \\
\hline $100 \mathrm{ml} 0.9 \%$ sodium chloride solution & 0.30 & Local NHS contract cost \\
\hline Teicoplanin $400 \mathrm{mg}$ injection & 35.62 & BNF 50 \\
\hline Cefuroxime $1.5 \mathrm{~g}$ injection & 4.70 & BNF 50 \\
\hline Cefuroxime $750 \mathrm{mg}$ injection & 2.34 & BNF 50 \\
\hline Water for injection $10 \mathrm{ml}$ & 0.31 & BNF 50 \\
\hline Cost per orthopaedic stay including hip arthroplasty & 6060.60 & NHS reference costs; http://www.dh.gov.uk/ \\
\hline Inpatient day for orthopaedic surgical patient & 200.00 & NHS reference costs \\
\hline MRSA test & 7.09 & NHS reference costs \\
\hline Barrier nursing $^{a}$ & 348.27 & Kunori, $2002^{104}$ \\
\hline Wound exploration ${ }^{b}$ & 1107.00 & NHS reference costs; http://www.dh.gov.uk/ \\
\hline Rifampicin 300 mg capsule & 0.60 & BNF 50 \\
\hline Erythromycin $500 \mathrm{mg}$ tablets & 0.19 & BNF 50 \\
\hline
\end{tabular}


TABLE $2 I$ Effect of SSI on cost, QALYs and the number of days' use of glycopeptides

\begin{tabular}{|lccccccccc|}
\hline Event & $\begin{array}{c}\text { Cost } \\
(\boldsymbol{E})\end{array}$ & QALYs & $\begin{array}{c}\text { Glycopeptide } \\
\text { use (days) }\end{array}$ & Sub-event & Probability & $\begin{array}{c}\text { Cost } \\
(\boldsymbol{t})\end{array}$ & $\begin{array}{c}\text { QALYs } \\
\text { Glycopeptide } \\
\text { use (days) }\end{array}$ \\
\hline No infection & 6,061 & 8.53 & 0.00 & & & & & & \\
MRSA infection & $\mathrm{II}, 104$ & 7.44 & 7.09 & Superficial & 0.807 & 11,177 & 8.24 & 7 \\
& & & & & Deep/joint & 0.103 & 20,235 & 7.70 & 14 \\
Other infection & 7,547 & 7.44 & 0.00 & Superficial & 0.807 & 7,853 & 8.24 & 0 \\
& & & & & Deep/joint & 0.103 & 11,745 & 7.70 & 0
\end{tabular}

TABLE 22 Effect of treatment options on cost, QALYs and days' use of glycopeptide

\begin{tabular}{|c|c|c|c|c|c|c|c|c|c|}
\hline \multirow[b]{2}{*}{ Option } & & \multirow[b]{2}{*}{$\begin{array}{c}\text { Cost } \\
(f)\end{array}$} & \multirow[b]{2}{*}{ QALYs } & \multirow[b]{2}{*}{$\begin{array}{l}\text { Glycopeptide } \\
\text { use (days) }\end{array}$} & \multicolumn{4}{|c|}{ Individual events (mutually exclusive) } & \multirow[b]{2}{*}{$\begin{array}{c}\text { Glycopeptide } \\
\text { use (days) }\end{array}$} \\
\hline & & & & & Event & Probability & $\begin{array}{c}\text { Cost } \\
(E)\end{array}$ & QALYs & \\
\hline Vancomycin & $\begin{array}{l}\text { Treatment } \\
\text { Event } \\
\text { Total }\end{array}$ & $\begin{array}{l}32.82 \\
6,086 \\
6,119\end{array}$ & $\begin{array}{l}0 \\
8.52 \\
8.52\end{array}$ & $\begin{array}{l}1 \\
0.01 \\
1.01\end{array}$ & $\begin{array}{l}\text { No infection } \\
\text { MRSA alone } \\
\text { Other alone } \\
\text { MRSA + other }\end{array}$ & $\begin{array}{r}0.986 \\
0.001 \\
0.012 \\
r \quad 0.000\end{array}$ & $\begin{array}{r}6,061 \\
11,104 \\
7,547 \\
11,104\end{array}$ & $\begin{array}{l}8.53 \\
7.44 \\
7.44 \\
7.44\end{array}$ & $\begin{array}{l}0.00 \\
7.09 \\
0.00 \\
7.09\end{array}$ \\
\hline Cephalosporin & $\begin{array}{l}\text { Treatment } \\
\text { Event } \\
\text { Total }\end{array}$ & $\begin{array}{l}19.73 \\
6,101 \\
6,120\end{array}$ & $\begin{array}{l}0 \\
8.52 \\
8.52\end{array}$ & $\begin{array}{l}0 \\
0.04 \\
0.04\end{array}$ & $\begin{array}{l}\text { No infection } \\
\text { MRSA alone } \\
\text { Other alone } \\
\text { MRSA + other }\end{array}$ & $\begin{array}{r}0.985 \\
0.005 \\
0.010 \\
r \quad 0.000\end{array}$ & $\begin{array}{r}6,061 \\
11,104 \\
7,547 \\
11,104\end{array}$ & $\begin{array}{l}8.53 \\
7.44 \\
7.44 \\
7.44\end{array}$ & $\begin{array}{l}0.00 \\
7.09 \\
0.00 \\
7.09\end{array}$ \\
\hline $\begin{array}{l}\text { Cephalosporin } \\
\text { and } \\
\text { vancomycin }\end{array}$ & $\begin{array}{l}\text { Treatment } \\
\text { Event } \\
\text { Total }\end{array}$ & $\begin{array}{l}52.55 \\
6,082 \\
6,135\end{array}$ & $\begin{array}{l}0 \\
9 \\
8.52\end{array}$ & $\begin{array}{l}0 \\
0.01 \\
0.01\end{array}$ & $\begin{array}{l}\text { No infection } \\
\text { MRSA alone } \\
\text { Other alone } \\
\text { MRSA + other }\end{array}$ & $\begin{array}{r}0.989 \\
0.001 \\
0.010 \\
r \quad 0.000\end{array}$ & $\begin{array}{r}6,061 \\
11,104 \\
7,547 \\
11,104\end{array}$ & $\begin{array}{l}8.53 \\
7.44 \\
7.44 \\
7.44\end{array}$ & $\begin{array}{l}0.00 \\
7.09 \\
0.00 \\
7.09\end{array}$ \\
\hline
\end{tabular}

summarises the effect of the different treatment options on costs, QALYs and the average (mean) number of days' use of glycopeptides. This table also includes data on the probability of infection for each type of treatment option. Both tables summarise the data detailed in the previous three sections.

The incremental cost-effectiveness analysis examined the relative cost-effectiveness of vancomycin, cephalosporin and a combination of the cephalosporin with vancomycin. The indicative model was populated with the data detailed in Tables 21 and 22. Assuming a cost-effectiveness threshold of $£ 30,000$ per QALY gained, an expected net benefit for each option was calculated for a range of baseline MRSA SSI rates and non-MRSA rates: $0-1.0 \%$ and $0-3.0 \%$, respectively. This analysis identified the optimal choice of prophylaxis (vancomycin, cephalosporin or cephalosporin and vancomycin) at each level of infection rates, as summarised in Table 23.
The purpose of the analysis is to show the sort of modelling that could be undertaken if the complexities and evidence limitations discussed in the section 'Purpose' (p. 64) can be overcome. However, the relationship between the indicative results presented here and underlying infection risk is useful to understand. These results suggest that a cephalosporin alone is only optimal (1) when the other infection rate is $0 \%$ or (2) when the MRSA infection rate is $\geqslant 0.2 \%$ and the other infection rate is $\leqslant 0.1 \%$. Vancomycin alone is only optimal (1) where the MRSA infection rate is $\leqslant 0.15 \%$ and the other infection rate is $0.1 \%$ or (2) if the MRSA infection rate is $\leqslant 0.2 \%$ and the other infection rate is $\geqslant 0.2 \%$. If the MRSA infection rate is $\geqslant 0.25 \%$ and the other infection rate is $\geqslant 0.2 \%$, the combination of cephalosporin plus vancomycin is optimal.

The total glycopeptide use for each treatment option was also estimated as reported in Table 24. This showed that, up to a baseline MRSA infection rate of between 20 and $25 \%$, use of antibiotic 
TABLE 23 Details of the optimal form of prophylaxis [vancomycin (V), cephalosporin (C) or cephalosporin plus vancomycin (CV)] for a given combination of baseline MRSA infection rate and other infection rate, assuming a threshold cost-effectiveness of $£ 30,000$ per QALY gained

\begin{tabular}{|c|c|c|c|c|c|c|c|c|c|c|c|c|c|c|}
\hline \multirow{2}{*}{$\begin{array}{l}\text { Other } \\
\text { infection } \\
\text { rate: CV } \\
\text { (\%) }\end{array}$} & \multicolumn{14}{|c|}{ MRSA infection rate (\%) } \\
\hline & 0 & 0.05 & 0.10 & 0.15 & 0.20 & 0.25 & 0.30 & 0.40 & 0.50 & 0.60 & 0.70 & 0.80 & 0.90 & 1.00 \\
\hline 0.0 & C & C & C & C & C & C & C & C & C & C & C & C & C & C \\
\hline 0.1 & V & V & $V$ & $\mathrm{~V}$ & C & C & C & C & C & C & C & C & C & C \\
\hline 0.2 & V & V & V & V & V & $\mathrm{CV}$ & $\mathrm{CV}$ & $\mathrm{CV}$ & $\mathrm{CV}$ & $\mathrm{CV}$ & $C V$ & $\mathrm{CV}$ & $\mathrm{CV}$ & $C V$ \\
\hline 0.3 & V & V & V & V & V & $\mathrm{CV}$ & $\mathrm{CV}$ & $\mathrm{CV}$ & $\mathrm{CV}$ & $\mathrm{CV}$ & $\mathrm{CV}$ & $\mathrm{CV}$ & $\mathrm{CV}$ & $\mathrm{CV}$ \\
\hline 0.4 & V & V & V & V & V & $\mathrm{CV}$ & $\mathrm{CV}$ & $\mathrm{CV}$ & $\mathrm{CV}$ & $\mathrm{CV}$ & $C V$ & $\mathrm{CV}$ & $\mathrm{CV}$ & $C V$ \\
\hline 0.5 & V & V & V & V & V & $\mathrm{CV}$ & $\mathrm{CV}$ & $\mathrm{CV}$ & $\mathrm{CV}$ & $\mathrm{CV}$ & $\mathrm{CV}$ & $\mathrm{CV}$ & $\mathrm{CV}$ & $\mathrm{CV}$ \\
\hline 1.0 & V & V & V & V & V & $\mathrm{CV}$ & $\mathrm{CV}$ & $\mathrm{CV}$ & $\mathrm{CV}$ & $\mathrm{CV}$ & $\mathrm{CV}$ & $\mathrm{CV}$ & $\mathrm{CV}$ & $\mathrm{CV}$ \\
\hline 1.5 & V & V & V & V & V & $\mathrm{CV}$ & $\mathrm{CV}$ & $\mathrm{CV}$ & $\mathrm{CV}$ & $\mathrm{CV}$ & $\mathrm{CV}$ & $\mathrm{CV}$ & $\mathrm{CV}$ & $\mathrm{CV}$ \\
\hline 2.0 & V & V & V & V & V & $\mathrm{CV}$ & $\mathrm{CV}$ & $\mathrm{CV}$ & $\mathrm{CV}$ & $\mathrm{CV}$ & $\mathrm{CV}$ & $\mathrm{CV}$ & $\mathrm{CV}$ & $\mathrm{CV}$ \\
\hline 2.5 & V & V & V & V & V & $\mathrm{CV}$ & $C V$ & $\mathrm{CV}$ & $\mathrm{CV}$ & $\mathrm{CV}$ & $\mathrm{CV}$ & $\mathrm{CV}$ & $\mathrm{CV}$ & $\mathrm{CV}$ \\
\hline 3.0 & V & V & V & V & V & $\mathrm{CV}$ & $\mathrm{CV}$ & $\mathrm{CV}$ & $\mathrm{CV}$ & $C V$ & $\mathrm{CV}$ & $\mathrm{CV}$ & $\mathrm{CV}$ & $\mathrm{CV}$ \\
\hline
\end{tabular}

TABLE 24 Expected use for each treatment option

\begin{tabular}{|lccccccccccccc}
\hline & \multicolumn{10}{c|}{ Baseline MRSA rate (\%) } \\
\cline { 2 - 12 } & $\mathbf{0}$ & $\mathbf{5}$ & $\mathbf{1 0}$ & $\mathbf{1 5}$ & $\mathbf{2 0}$ & $\mathbf{2 5}$ & $\mathbf{3 0}$ & $\mathbf{3 5}$ & $\mathbf{4 0}$ & $\mathbf{4 5}$ & $\mathbf{5 0}$ \\
\hline $\begin{array}{l}\text { Expected glycopeptide use with } \\
\text { vancomycin prophylaxis }\end{array}$ & 0 & 0.354 & 0.709 & 1.064 & 1.418 & 1.773 & 2.127 & 2.482 & 2.836 & 3.191 & 3.546 \\
$\begin{array}{l}\text { Expected glycopeptide use with } \\
\text { cephalosporin prophylaxis }\end{array}$ & 1 & 1.100 & 1.207 & 1.323 & 1.449 & 1.586 & 1.737 & 1.902 & 2.083 & 2.285 & 2.510 \\
\hline
\end{tabular}

prophylaxis is not glycopeptide sparing. That is, up to these rates, the total use of glycopeptides (for prophylaxis and for treatment) is greater than when glycopeptides are used only for treatment. This might be expected to have significant implications for drug resistance over the longer term.

\section{Discussion}

This study set out to examine whether there is a threshold value for prevalence of MRSA that favours routine prophylaxis with glycopeptide antibiotics.

In order to answer this question, we developed an economic model, using hip arthroplasty by way of an exemplar. However, it is clear from the clinical evidence presented in Chapter 3 and the economic reviews in Chapters 4 and 5 that such a modelling exercise could only be indicative in nature. To address fully the cost-effectiveness of glycopeptides as surgical antibiotic prophylaxis gathering, synthesis and modelling initiative than has been possible in this review. The characteristics of such a research programme are described in Chapter 7.

Despite the model being indicative rather than definitive, it shows the approach that would be possible given appropriate evidence. It can be used to show the threshold baseline risk of MRSA infection at which a particular intervention (here glycopeptides as prophylaxis) might be costeffective. This involves estimating a treatment effect of the intervention on that baseline risk, incorporating its acquisition cost and those of its comparators and quantifying the implications of the various types of infections in terms of resource costs and quality-adjusted survival duration. An important feature of this modelling framework is the interpretation of the baseline risk threshold. As presented here, it relates to an average risk of MRSA SSI in the population of patients undergoing hip arthroplasty in a given centre. However, this baseline risk can also be seen at the level of the individual patient. Using a multivariable risk model, the baseline risk of 
infection in an individual patient can be predicted based on patient-level characteristics such as previous infections, age and co-morbidities such as presence of decubitus ulcer or history of previous hospitalisation, in addition to centre-level characteristics. ${ }^{109}$ Such an interpretation can also incorporate the effect of MRSA screening, which effectively changes a baseline (prior) probability of infection based on a population average into a revised (posterior) probability of infection.

Although the model results presented here are of little direct policy relevance, the indicative model suggests that, where the MRSA infection rate is $\geqslant 0.25 \%$ and the rate of other infections with cephalosporin prophylaxis is $\geqslant 0.2 \%$, the combination of cephalosporin plus vancomycin is the optimal antibiotic prophylaxis for hip arthroplasty patients. These results are not very surprising because vancomycin is very similar in cost to the standard cephalosporin, cefuroxime, but the limited evidence available seems to suggest that vancomycin is more effective in preventing MRSA infections. Furthermore, the combination arm has been assumed to have an additive effect. There is a mortality reduction associated with infection rate reduction. The cost of the antibiotics alone contributed little to overall costs and there was relatively little difference in cost across antibiotics used. In MRSA-infected patients, hospital discharge was delayed between 8 and 23 days, on average, with substantial cost implications. Therefore, a small decrease in attributable mortality would, on average, typically be expected to offset the cost of the antibiotic prophylaxis. Therefore, by reducing the rate of MRSA infection, vancomycin generates a gain in quality-adjusted life expectancy compared with cefuroxime; the combination strategy generates an even greater gain. As a result, in the indicative model, the baseline risk of MRSA can be modest (below the national average) and it would still appear cost-effective to use glycopeptides.

However, this conclusion is reached in the absence of any explicit modelling of the effect of greater use of glycopeptides on drug resistance. Little is known about current levels of resistance, the rate of development of resistance transmission rates and the impact of interventions on resistance. Therefore, like all modelling undertaken in this and related areas (see Chapters 4 and 5), no attempt has been made explicitly to reflect future resistance in the model. The model does indicate that, at all plausible baseline infection rates, the use of glycopeptides as a form of prophylaxis, in addition to a treatment of infections, will increase the total use of the drug. Therefore, even if the argument is accepted that the risk of resistance is directly proportional to total antibiotics usage and the argument that the shorter courses of antibiotics used in prophylaxis are more likely to lead to resistance than the longer therapeutic courses is discounted, prophylactic use of glycopeptides is unlikely to decrease total usage and so decrease the risk of resistance problems in the future. 



\section{Chapter 7 Discussion}

$\mathrm{T}$ he aim of this project was to determine whether there is a level of MRSA prevalence at which a switch from non-glycopeptide to glycopeptide antibiotics for routine prophylaxis is indicated in surgical environments with a high risk of MRSA infection. To answer this question, a systematic review of the clinical effectiveness and cost-effectiveness of glycopeptide prophylaxis was undertaken, and a decision analytic model investigating the cost-effectiveness of glycopeptides in relation to MRSA prevalence was developed. The systematic reviews provided little evidence for the clinical effectiveness or costeffectiveness of glycopeptides for MRSA prophylaxis. No cost-effectiveness studies and only two trials were conducted in the UK, which limits their generalisability. There was also a lack of recent evidence, as most of the studies were conducted prior to 2001. This confirms the conclusions of Glenny and Song, who conducted a systematic review in 1998 of antimicrobial prophylaxis for total hip replacement and concluded that the volume of research in this area was decreasing. ${ }^{110}$

Most studies identified in the effectiveness review did not report the prevalence of MRSA or the incidence of MRSA infections as an outcome. With one exception, a trial of cardiac surgery conducted in a hospital in Israel with a high MRSA prevalence, ${ }^{17}$ the trials were not designed primarily to address MRSA prevention. This meant that the systematic review was unable to link the effectiveness of glycopeptide prophylaxis to different MRSA prevalence levels.

It was not possible to undertake definitive modelling of cost-effectiveness within the scope of this project, given the lack of clinical evidence and major complexities, such as the choice of prophylaxis and treatment options and the highlevel uncertainty about future development of resistance. Instead, an indicative cost-effectiveness model was developed which focused on a single surgical specialty. Hip arthroplasty was chosen as an exemplar, as it is a clean procedure, patients are at high risk of infection (including MRSA), and it was expected that more data would be available compared with other surgical specialties. However, this model is indicative only and considers vancomycin but not teicoplanin prophylaxis. Given that the relevant evidence is so scant and the problem of modelling resistance so complex, this emphasises the care required in formalising policy in the context of resistance.

It is hoped that this indicative modelling framework will contribute to future research in this area in a number of ways. It shows how available evidence on a range of baseline infection risks, acquisition costs and treatment effects of alternative anti-infection interventions, and the costs and health consequences of infection can be brought together to inform decisions about the relative cost-effectiveness of these options as a function of baseline risks. Although the indicative model here was developed in the exemplar area of hip arthroplasty, the framework has relevance to a range of surgical areas including other 'clean' procedures such as vascular surgery, which, like orthopaedic surgery, is high-volume surgery and is increasingly performed in older, sicker patients who are more likely to be MRSA carriers. The model structure would have to be adjusted in contaminated procedures such as gastrointestinal surgery, where the presence of bacteria means that patients may be classified as 'infected' prior to the procedure.

Our indicative model concentrates on the use of vancomycin compared with cephalosporin prophylaxis in primary hip arthroplasty. However, most orthopaedic procedures are now carried out in specialist 'clean-air' suites that reduce the need for anti-MRSA prophylaxis. This is not necessarily the case for other types of surgery. Joint replacement patients are considered to be 'highrisk' patients, as an infection could have serious long-term consequences requiring revision surgery. Despite patient, manager and infection control teams' concerns about MRSA, this may not be the major concern for surgeons in orthopaedic surgery as evidence suggests that MRSE infections are more of a problem in failed joint replacements ${ }^{101}$ (Kay P, Campbell P, Consultant Orthopaedic Surgeons: personal communication, 2006) suggesting that focusing on MRSA may be too narrow a focus in areas other than orthopaedic surgery. 
More fundamentally, the mechanisms of development of resistance may be affected by the type of surgical procedure. The use of vancomycin in gastrointestinal surgery has been reported to promote the development of VRE, and this resistant strain is known to pass on its genes to S. aureus, thus cultivating VRSA. ${ }^{111,112}$ This mechanism has not been reported in clean surgery. The supplementary reviews conducted to assist with the modelling of antibiotic resistance found indicative models only where the methods varied according to disease type. In addition, no models were identified which could prospectively predict changes in the rate of the development of resistance in the global clinical population resulting from changes in treatment practice. These reviews did not provide any insights into how to model cost-effectiveness in the context of resistance. Given the constraints of the project, we were unable to incorporate the emergence of glycopeptide resistance in our model, and so could not fully explore its impact upon the use of glycopeptides as prophylaxis. However, our model indicates that, over all baseline MRSA prevalence rates, increasing the use of glycopeptide prophylaxis is likely to increase the risk of future resistance problems, if we assume that increased environmental exposure to glycopeptides is one of the factors that increase prevalence of resistance.

\section{Development of a model to guide surgical antibiotic prophylaxis prescribing}

The research need that led to this project was the development of a model that identifies an MRSA prevalence rate, or series of rates, dependent on other risk variables, which will inform practitioners whether a particular set of patient, environmental and procedural variables suggest that a patient, or patient group, should have glycopeptide surgical prophylaxis.

Development of a model or algorithm which could be used by a clinician to guide surgical antibiotic prophylaxis prescribing would have substantial clinical application. The combination of reviews and modelling carried out in this study suggests that such a model would require a number of inputs. Current uncertainty about the nature of those inputs, instability of these over time and complexity of the relationship of those inputs suggest that the development of a full model, or clinical algorithm, would require a groups. Practically, it would be difficult to define an MRSA threshold for a particular hospital. Discussion with clinical experts has suggested that an individual patient's risk of infection would be more useful for decision-making as the idea that a whole specialty would change its prophylaxis policy for all patients on the basis of an average infection rate, which may be out of date by the time the decision is taken, may be unrealistic. The hospital's ward-based and perioperative infection control policies, including MRSA screening, are factors affecting an individual patient's risk of an infection. If a hospital has a high MRSA prevalence, this is indicative of a failure of infection control systems, and suggests that they need to take other actions to improve those systems. If rates are high, or there is an outbreak, then wards or surgeries should be temporarily closed; increasing glycopeptide use will not be the solution. A policy or clinical algorithm should not just advocate the use of glycopeptide prophylaxis in this scenario.

\section{Recommendations for practice}

Due to the lack of available evidence about the effectiveness of glycopeptide prophylaxis, and complexities relating to the range of potential management options, we have been unable to develop a definitive decision analytic model which could be used by surgical centres to guide antibiotic prophylaxis choice. Our indicative model of the choice between vancomycin and cephalosporin prophylaxis in hip arthroplasty illustrates when vancomycin is likely to be most cost-effective based on varying baseline MRSA and other non-MRSA infection rates. However, this is an illustrative model only and inevitably provides only limited policy guidance. It does show that the use of glycopeptide prophylaxis is unlikely to reduce future overall exposure and may increase the risk of future resistance problems. The new British Society for Antimicrobial Chemotherapy guidelines ${ }^{4}$ recommend that glycopeptides (alone or with other antibiotics) should be given to patients with a history of MRSA colonisation, or infections without documented eradication, or have come from a facility with a high prevalence of MRSA. This patient-based risk approach, combined with knowledge of local resistance patterns, may be the most appropriate approach to use of glycopeptides in surgical prophylaxis, given current uncertainties around the effectiveness of glycopeptides, development of resistance and impact of glycopeptides on resistance patterns. 


\section{Recommendations for research}

This review highlights the complexities of decision-making relating to infection control in general and of MRSA control in particular. As yet, the full mechanism of resistance is not fully understood. Microbiologists and epidemiologists in the field are likely to provide an important contribution in furthering this understanding. Their research expertise could provide a useful precursor to further economic modelling work.

- Research to inform decisions in this area needs to reflect these complexities and adopt analytical methods which can handle them. One implication of this is that a focus on MRSA, rather than a broader consideration of infections in general, is too limited. Although the media may present it as otherwise, there seems to be little basis to justify MRSA being a priority in terms of prevalence or the severity of its sequelae, and future research needs to place MRSA in a broader context of infection control. Similarly, as highlighted in the fourth literature review in Chapter 5 ('A conceptual framework for the economic evaluation of policies against MRSA', p. 56), there is a large number of possible infection control policies which can be used in the presence of infection problems (MRSA or otherwise), and the prophylactic use of glycopeptides is only one of these. The results of this project suggest that the clinical evidence about glycopeptides in this context is limited. An investigation of whether other infection control policies would be more effective and costeffective than prophylaxis is needed.

- A full evidence synthesis and modelling study to inform wider decision-making in infection control is warranted, given a suitable budget and timetable. Such a study would aim to define the complete range of interventions and polices used in infection control for MRSA but also extending to other infections. This would need to include hospital- or department-wide policies such as bed configuration and screening and also interventions for individual patients such as prophylactic antibiotics. It would be necessary to bring together a wider evidence base to inform this comparison, and to include suitably elicited opinion from appropriate experts. The use of interdisciplinary collaboration would help to pursue the research agenda and to make use of the wide evidence base. For example, expertise developed in biostatistical modelling, such as in HIV/AIDS, could be highly relevant, in terms of both methods and sources of data used. The results from this research would inform both policy and priorities for primary research in this area.

- As this review has highlighted, a lack of evidence about the clinical effectiveness of glycopeptide prophylaxis for MRSA, a large multi-centre trial or cluster randomised trial is needed to address effectiveness across surgical centres with varying levels of MRSA prevalence. This could then be used to aid the development of a full decision analytic model. However, as rates of post-surgical MRSA infections are generally very low $(<1 \%)$, sample sizes would have to be large (for example, to detect a reduction in MRSA rates from 1 to $0.5 \%$ at a power of $80 \%$ would require approximately 9000 patients). An alternative strategy would be to perform a cluster randomised trial, with surgical units being randomised to a particular regimen rather than individual patients, although this would require a larger sample size than an equivalent patient-level RCT. As such a trial may be difficult to conduct, a feasibility project could be undertaken by surveying surgeons across different specialties to identify if they would be willing to participate.

- However, such a trial may not be the best option for future research as by the time the results were available they may no longer be of importance in the light of new anti-MRSA treatments currently in development. Given the paucity of clinical effectiveness data and the problems that undertaking trials in this area may present, it would be worth devoting research effort to see whether other non-experimental data could provide useful information.

- Measuring the development and patterns of resistance to glycopeptides requires further long-term research over many years and surveillance of the incidence of glycopeptideresistant bacteria, rather than through the medium of RCTs, which are suited to hypothesis testing, not characterisation or examination of complex longitudinal mechanisms. A research programme to predict the long-term pattern of drug resistance, its implications for future costs and health and the inter-sectoral, macroeconomic and international effects relating to resistance is needed. Modelling resistance is further complicated by the fact that there are numerous strains of MRSA and they all develop resistance using different mechanisms. Such a research initiative would be relatively expensive and take some years to produce useful results, but it is essential before major additional resources are devoted to new primary research such as RCTs. 



\section{Chapter 8 Conclusions}

$\mathrm{T}$ here is not enough evidence available from RCTs to assist in decision-making about whether and when to change from nonglycopeptide to glycopeptide antibiotics for prophylaxis. There was also a lack of evidence about the cost-effectiveness of glycopeptide antibiotic prophylaxis.

Due to the lack of available evidence about the effectiveness of glycopeptide prophylaxis, and complexities relating to the range of potential management options, we were unable to develop a definitive decision analytic model. The indicative economic model developed using hip arthroplasty as a surgical exemplar shows how available evidence on a range of baseline infection risks, the acquisition costs and treatment effects of alternative anti-infection interventions and the costs and health consequences of infection can be brought together to inform decisions about the relative cost-effectiveness of these options as a function of baseline risks. The findings of the indicative model do not support the case for using glycopeptides prophylactically rather than therapeutically.

There is a lack of available trial data reporting the incidence of glycopeptide-resistant bacteria, and no economic models incorporating resistance were identified. The impact of antibiotic resistance on the wider population was not incorporated into the economic model. There is very large uncertainty in terms of the impact of antibiotic resistance, surgeons and hospitals risk aversion to resistance and substantial variation in hospital infection control procedures across the UK, all of which would need to be incorporated into a 'full' economic model. The modelling of bacterial resistance is an extremely complex issue encompassing the effects of time, drug use and patterns of transmission and was beyond the scope of this project, given its short duration. 



\section{8 \\ Acknowledgements}

$\mathrm{W}$ e would like to thank our expert advisors for their advice during this project, and for providing comments on the protocol and report. They were Peter Campbell (Consultant Orthopaedic Surgeon), Professor Peter Gilbert (Clinical Microbiologist), Dr Ian Gould (Consultant Microbiologist), Peter Kay (Consultant Orthopaedic Surgeon), Steve Leveson (General Surgeon) and Jonathon Michaels (Professor of Vascular Surgery), and Eimear NicLochlainn, John Edmunds, Jenny Roberts and Richard Smith who advised on modelling methods. We would also like to thank our colleagues at the University of York, Professor Martin Bland, Professor Eve Roman and Professor David Torgerson, for providing advice on potential study designs for future research.

\section{Contribution of authors}

Gillian Cranny (Research Fellow) worked on the systematic review of effectiveness and was responsible for writing the protocol, study selection, data extraction, validity assessment and report writing. Rachel Elliott (Clinical Senior Lecturer) was responsible for the cost-effectiveness review of non-glycopeptide antibiotic prophylaxis for surgery and the cost-effectiveness review of antibiotics where antibiotic resistance is a problem. She was involved in the selection of studies, data extraction, quality assessment and report writing, and also in the development of the economic model. She contributed clinical and pharmacological expertise to the background section and the effectiveness section. Helen Weatherly (Research Fellow) was responsible for the cost-effectiveness review of glycopeptide antibiotics compared with appropriate comparators. She was involved in the selection of studies, data extraction and quality assessment, all the cost-effectiveness reviews, report writing and in the development of the economic model. Duncan Chambers (Research Fellow) worked on the systematic review of effectiveness and was responsible for writing the protocol, study selection, data extraction, validity assessment and report writing. Neil Hawkins (Research Fellow) was responsible for the review of the use of epidemiological and decision-analytic techniques to model antibiotic resistance in addition to being responsible for the economic model. He was involved in the selection of studies, data extraction and report writing. Lindsey Myers (Information Officer) devised the search strategy, carried out the literature searches and contributed to report writing. Mark Sculpher (Professor of Health Economics) provided input at all stages. He was responsible for writing the section on a conceptual framework for the economic evaluation of policies against MRSA and the discussion sections. He commented on the design of the economic model and on various drafts of the report. Alison Eastwood (Senior Research Fellow) provided input at all stages, commented on various drafts of the report and had overall responsibility for the clinical effectiveness review. 



\section{References}

1. Plowman R, Graves N, Griffin M, Roberts JA, Swan AV, Cookson B, et al. The socio-economic burden of hospital acquired infection. London: Public Health Laboratory Service; 1999.

2. Coello R, Charlett A, Wilson J, Ward V, Pearson A, Borriello P. Adverse impact of surgical site infections in English hospitals. J Hosp Infect 2005;60:93-103.

3. Scottish Intercollegiate Guidelines Network. Antibiotic prophylaxis in surgery SIGN 45. Edinburgh: Scottish Intercollegiate Guidelines Network; 2000.

4. Gemmell CG, Edwards DI, Fraise AP, Gould FK, Ridgway GL, Warren RE. Guidelines for the prophylaxis and treatment of methicillin-resistant Staphylococcus aureus (MRSA) infections in the UK. J Antimicrob Chemother 2006;57:589-608.

5. Nosocomial Infection National Surveillance Service. Surveillance of surgical site infection in English hospitals 1997-2002. London: Public Health Laboratory Service, 2002.

6. Mangram AJ, Horan TC, Pearson ML, Silver LC, Jarvis WR. Guideline for the prevention of surgical site infection. Infect Control Hosp Epidemiol 1999;20:250-78.

7. Tenover FC, Biddle JW, Lancaster MV. Increasing resistance to vancomycin and other glycopeptides in Staphylococcus aureus. Emerg Infect Dis 2001;7:327-32.

8. Chang S, Sievert DM, Hageman JC, Boulton ML, Tenover FC, Downes FP, et al. Infection with vancomycin-resistant Staphylococcus aureus containing the vanA resistance gene. $N$ Engl J Med 2003;348:1342-7.

9. Aucken HM, Ganner M, Murchan S, Cookson BD, Johnson AP. A new UK strain of epidemic methicillin-resistant Staphylococcus aureus (EMRSA17) resistant to multiple antibiotics. J Antimicrob Chemother 2002;50:171-5.

10. Bertrand X, Hocquet D, Thouverez M, Plesiat P, Talon D. Characterisation of methicillin-resistant Staphylococcus aureus with reduced susceptibility to teicoplanin in Eastern France. Eur J Clin Microbiol Infect Dis 2003;22:504-6.

11. Movahed MR, Kasravi B, Bryan CS. Prophylactic use of vancomycin in adult cardiology and cardiac surgery. J Cardiovasc Pharmacol Ther 2004;9:13-20.

12. NHS Centre for Reviews and Dissemination. Undertaking systematic reviews of research on effectiveness: CRD's guidance for those carrying out or commissioning reviews. CRD Report 4. 2nd ed. York: University of York; 2001.

13. Codina C, Miro JM, Tuset M, Claramonte J, Gomar C, Gotsens R, et al. [Vancomycin and teicoplanin use as antibiotic prophylaxis in cardiac surgery: pharmacoeconomic study]. Med Clin 2000;114 Suppl 3:54-61; in Spanish.

14. Caprioli S, Jelmoni GP, Perle S, Legnani P, Maggiolo F, Suter F. Vancomycin plus centamycin vs cefamandole in prosthetic orthopedic surgery prophylaxis. Can J Infect Dis 1995;6 Suppl C:2011.

15. Miro JM, Pomar JL, Claramonte X, Mestres C, Cartana R, Gatell JM, et al. Vancomycin (Van) versus teicoplanin (Tei) for antibiotic prophylaxis in cardiac surgery. Program and Abstracts of the Interscience Conference on Antimicrobial Agents and Chemotherapy 1996;36:248.

16. Kitzis M, Branger C, Andreassian B. Antimicrobial prophylaxis with vancomycin a comparative study in vascular surgery. In Interscience Conference on Antimicrobial Agents and Chemotherapy. Program and Abstracts of the Thirty-first Interscience Conference on Antimicrobial Agents and Chemotherapy; 1991 Sept 29-Oct 2; Chicago, Illinois. Washington, DC: American Society for Microbiology; 1991. p. 254.

17. Finkelstein R, Rabino G, Mashiah T, Bar-El Y, Adler Z, Kertzman V, et al. Vancomycin versus cefazolin prophylaxis for cardiac surgery in the setting of a high prevalence of methicillin-resistant staphylococcal infections.J Thorac Cardiovasc Surg 2002;123:326-32.

18. Saginur R, Croteau D, Bergeron MG. Comparative efficacy of teicoplanin and cefazolin for cardiac operation prophylaxis in 3027 patients. J Thorac Cardiovasc Surg 2000;120:1120-30.

19. Salminen U-S, Viljanen TU, Valtonen VV, Ikonen TE, Sahlman AE, Harjula AL. Ceftriaxone versus vancomycin prophylaxis in cardiovascular surgery. J Antimicrob Chemother 1999;44:287-90.

20. Vuorisalo S, Pokela R, Syrjala H. Comparison of vancomycin and cefuroxime for infection prophylaxis in coronary artery bypass surgery. Infect Control Hosp Epidemiol 1998;19:234-9.

21. Maki DG, Bohn MJ, Stolz SM, Kroncke GM, Acher CW, Myerowitz PD. Comparative study of cefazolin, cefamandole, and vancomycin for surgical prophylaxis in cardiac and vascular operations. A double-blind randomized trial. J Thorac Cardiovasc Surg 1992;104:1423-34. 
22. Marroni M, Cao P, Fiorio M, Maghini M, Lenti M, Repetto A, et al. Prospective, randomized, doubleblind trial comparing teicoplanin and cefazolin as antibiotic prophylaxis in prosthetic vascular surgery. Eur J Clin Microbiol Infect Dis 1999; 18:175-8.

23. Kester RC, Antrum R, Thornton CA, Ramsden $\mathrm{CH}$, Harding I. A comparison of teicoplanin versus cephradine plus metronidazole in the prophylaxis of post-operative infection in vascular surgery. J Hosp Infect 1999;41:233-43.

24. Periti P, Stringa G, Mini E, Italian Study Group for Antimicrobial Prophylaxis in Orthopedic Surgery. Comparative multicenter trial of teicoplanin versus cefazolin for antimicrobial prophylaxis in prosthetic joint implant surgery. Eur J Clin Microbiol Infect Dis 1999;18:113-19.

25. Suter F, Avai A, Fusco U, Gerundini M, Caprioli S, Maggiolo F. Teicoplanin versus cefamandole in the prevention of infection in total hip replacement. Eur J Clin Microbiol Infect Dis 1994;13:793-6.

26. Mollan RA, Haddock M, Webb CH. Teicoplanin vs cephamandole for antimicrobial prophylaxis in prosthetic joint implant surgery: preliminary results. Eur J Surg Suppl 1992;567:19-21.

27. de Lalla F, Novelli A, Pellizzer G, Milocchi F, Viola $\mathrm{R}$, Rigon A, et al. Regional and systemic prophylaxis with teicoplanin in monolateral and bilateral total knee replacement procedures: study of pharmacokinetics and tissue penetration. Antimicrob Agents Chemother 1993;37:2693-8.

28. Pons VG, Denlinger SL, Guglielmo BJ, Octavio J, Flaherty J, Derish PA, et al. Ceftizoxime versus vancomycin and gentamicin in neurosurgical prophylaxis: a randomized, prospective, blinded clinical study. Neurosurgery 1993;33:416-23.

29. Ratto GB. Antibiotic prophylaxis in lung cancer surgery. Lung Cancer 1990;6:18-91.

30. Mercieri M, Mercieri A, Tritapepe L, Ruggeri M, Arcioni R, Repetto M, et al. High-dose aprotinin with gentamicin-vancomycin antibiotic prophylaxis increases blood concentrations of creatinine and cystatin $\mathrm{C}$ in patients undergoing coronary artery bypass grafting. Br J Anaesth 1999;82:531-6.

31. Romanelli VA, Howie MB, Myerowitz PD, Zvara DA, Rezaei A, Jackman DL, et al. Intraoperative and postoperative effects of vancomycin administration in cardiac surgery patients: a prospective, double-blind, randomized trial. Crit Care Med 1993;21:1124-31.

32. Bolon MK, Morlote M, Weber SG, Koplan B, Carmeli Y, Wright SB. Glycopeptides are no more effective than beta-lactam agents for prevention of surgical site infection after cardiac surgery: a metaanalysis. Clin Infect Dis 2004;38:1357-63.

33. Moher D, Shulz KF, Altman DG. The CONSORT statement: revised recommendations for improving the quality of reports of parallel-group randomised trials. Lancet 2001;357:1191-4.

34. Drummond MF, Sculpher MJ, Torrance GW, O'Brien BJ, Stoddart GL. Methods for the economic evaluation of health care programmes. 3rd ed. Oxford: Oxford University Press; 2005.

35. Phillips E, Louie M, Knowles SR, Simor AE, Oh PI. Cost-effectiveness analysis of six strategies for cardiovascular surgery prophylaxis in patients labeled penicillin allergic. Am J Health Syst Pharm 2000;57:339-45.

36. Spelman D, Harrington G, Russo P, Wesselingh S. Concise communications. Clinical, microbiological, and economic benefit of a change in antibiotic prophylaxis for cardiac surgery. Infect Control Hosp Epidemiol 2002;23:402-4.

37. Zanetti G, Goldie SJ, Platt R. Clinical consequences and cost of limiting use of vancomycin for perioperative prophylaxis: example of coronary artery bypass surgery. Emerg Infect Dis 2001;7:820-27.

38. Fryback DG, Dasbach EJ, Klein R, Klein BE, Dorn N, Peterson K, et al. The Beaver Dam Health Outcomes Study: initial catalog of health-state quality factors. Med Decis Making 1993;13:89-102.

39. National Institute for Clinical Excellence. Methodology checklist: economic evaluations. In Guideline development methods: information for national collaborating centres and guideline developers. London: National Institute for Clinical Excellence; 2004. pp. G1-6.

40. Ise Y, Hagiwara K, Saitoh S, Honjo K, Soh S, Kato A, et al. Comparison of the effects of prophylactic antibiotic therapy and cost effectiveness between cefazolin (CEZ) and sulbactam/ampicillin (SBT/ABPC) in gastric cancer surgery employing clinical pathway. Yakugaku Zasshi 2004;124:815-24.

41. Pearson CR, Thomas MR, Cox HJ, Garth RJN. A cost-benefit analysis of the post-operative use of antibiotic ear drops following grommet insertion. J Laryngol Otol 1996;110:527-30.

42. Blair EA, Johnson JT, Wagner RL, Carrau RL, Bizakis JG. Cost analysis of antibiotic prophylaxis in clean head and neck surgery. Arch Otolaryngol Head Neck Surg 1995;121:269-71.

43. VandenBergh MFQ, Kluytmans JAJW, van Hout BA, Maat APWM, Seerden RJ, McDonnel J, et al. Cost-effectiveness of perioperative mupirocin nasal ointment in cardiothoracic surgery. Infect Control Hosp Epidemiol 1996;17:786-92.

44. Pearle MS, Roehrborn CG. Antimicrobial prophylaxis prior to shock wave lithotripsy in patients with sterile urine before treatment: a meta-analysis and cost-effectiveness analysis. Urology 1997;49:679-86. 
45. Schilling J, Michalopoulos A, Geroulanos S. Antibiotic prophylaxis in gastroduodenal surgery. Hepatogastroenterology 1997;44:116-20.

46. Wieck JA, Jackson JK, O'Brien TJ, Lurate RB, Russell JM, Dorchak JD. Efficacy of prophylactic antibiotics in arthroscopic surgery. Orthopedics 1997;20:133-4.

47. Bold RJ, Mansfield PF, Berger DH, Pollock RE, Singletary SE, Ames FC, et al. Prospective, randomized, double-blind study of prophylactic antibiotics in axillary lymph node dissection. $\mathrm{Am} \mathrm{J}$ Surg 1998;176:239-43.

48. Kapoor DA, Klimberg IW, Malek GH, Wegenke JD, Cox CE, Patterson AL, et al. Single-dose oral ciprofloxacin versus placebo for prophylaxis during transrectal prostate biopsy. Urology 1998;52:552-8.

49. Kulling D, Sonnenberg A, Fried M, Bauerfeind P. Cost analysis of antibiotic prophylaxis for PEG. Gastrointest Endosc 2000;51:152-6.

50. Lazorthes F, Chiotasso P, Massip P, Materre JP, Sarkissian M. Local antibiotic prophylaxis in inguinal hernia repair. Surg Gynecol Obstet 1992;175:569-70.

51. Albers BA, Patka P, Haarman HJ, Kostense PJ. [Cost-effectiveness of preventive antibiotic administration for lowering risk of infection by 0.25\%]. Unfallchirurg 1994;97:625-8; in German.

52. Davey PG, Parker SE, Crombie IK, Jaderberg M. The cost effectiveness of amoxicillin/clavulanic acid as antibacterial prophylaxis in abdominal and gynaecological surgery. PharmacoEconomics 1995;7:347-56.

53. Pestotnik SL, Classen DC, Evans RS, Burke JP. Implementing antibiotic practice guidelines through computer-assisted decision support: clinical and financial outcomes. Ann Intern Med 1996;124:884-90.

54. Schwartz BF, Swanzy S, Thrasher JB. A randomized prospective comparison of antibiotic tissue levels in the corpora cavernosa of patients undergoing penile prosthesis implantation using gentamicin plus cefazolin versus an oral fluoroquinolone for prophylaxis. J Urol 1996;156:991-4.

55. Pavan MM, Malyuk DL. A cost effective approach to surgical antibiotic prophylaxis. Can J Hosp Pharm 1992;45:151-6.

56. Thomas R, Alvino P, Cortino GR, Accardo R, Rinaldo M, Pizzorusso M, et al. Long-acting versus short-acting cephalosporins for preoperative prophylaxis in breast surgery: a randomized double-blind trial involving 1,766 patients. Chemotherapy 1999;45:217-23.

57. Rau HG, Mittelkotter U, Zimmermann A, Lachmann A, Kohler L, Kullmann KH. Perioperative infection prophylaxis and risk factor impact in colon surgery. Chemotherapy 2000; 46:353-63.

58. Paladino JA, Rainstein MA, Serrianne DJ, Przylucki JE, Welage LS, Collura ML, et al. Ampicillin-sulbactam versus cefoxitin for prophylaxis in high-risk patients undergoing abdominal surgery. Pharmacotherapy 1994;14: 734-9.

59. Heit JM, Stevens MR, Jeffords K. Comparison of ceftriaxone with penicillin for antibiotic prophylaxis for compound mandible fractures. Oral Surg Oral Med Oral Pathol Oral Radiol 1997;83:423-6.

60. Hall JC, Christiansen K, Carter MJ, Edwards MG, Hodge AJ, Newman MA, et al. Antibiotic prophylaxis in cardiac operations. Ann Thorac Surg 1993;56:916-22.

61. Roach AC, Kernodle DS, Kaiser AB. Selecting costeffective antimicrobial prophylaxis in surgery: are we getting what we pay for? Drug Intell Clin Pharm 1990;24:183-5.

62. Fried GW, Goetz G, Potts-Nulty S, Solomon G, Cioschi HM, Staas WE. Prospective evaluation of antibiotic prophylaxis prior to cystometrogram and/or cystogram studies:oral versus intramuscular routes. Arch Phys Med Rehabil 1996;77:900-2.

63. Simpson KN, Luo MP, Chumney E, Sun E, Brun S, Ashraf T. Cost-effectiveness of lopinavir/ritonavir versus nelfinavir as the first-line highly active antiretroviral therapy regimen for HIV infection. HIV Clin Trials 2004;5:294-304.

64. Weinstein MC, Goldie SJ, Losina E, Cohen CJ, Baxter JD, Zhang H, et al. Use of genotypic resistance testing to guide HIV therapy: clinical impact and cost-effectiveness. Ann Intern Med 2001;134:440-50.

65. Corzillius M, Muhlberger N, Sroczynski G, Jaeger H, Wasem J, Siebert U. Cost effectiveness analysis of routine use of genotypic antiretroviral resistance testing after failure of antiretroviral treatment for HIV. Antiviral Ther 2004;9:27-36.

66. Sudre P, Breman JG, McFarland D, Koplan JP. Treatment of chloroquine-resistant malaria in African children: a cost-effectiveness analysis. Int J Epidemiol 1992;21:146-54.

67. Goodman CA, Coleman PG, Mills AJ. The costeffectiveness of antenatal malaria prevention in sub-Saharan Africa. Am J Trop Med Hyg 2001;64 Suppl 1-2:45-56.

68. Goodman CA, Coleman PG, Mills AJ. Changing the first line drug for malaria treatment - costeffectiveness analysis with highly uncertain intertemporal trade-offs. Health Econ 2001;10:731-49.

69. Coleman PG, Morel C, Shillcutt S, Goodman C, Mills AJ. A threshold analysis of the costeffectiveness of artemisinin-based combination 
therapies in sub-saharan Africa. Am J Trop Med Hyg 2004;71 Suppl 2:196-204.

70. Brewer TF, Heymann SJ, Ettling M. An effectiveness and cost analysis of presumptive treatment for Mycobacterium tuberculosis. Am J Infect Control 1998;26:232-8.

71. Weis SE, Foresman B, Matty KJ, Brown A, Blais FX, Burgess G, et al. Treatment costs of directly observed therapy and traditional therapy for Mycobacterium tuberculosis: a comparative analysis. Int J Tuberc Lung Dis 1999;3:976-84.

72. Drobniewski FA, Watterson SA, Wilson SM, Harris GS. A clinical, microbiological and economic analysis of a national service for the rapid molecular diagnosis of tuberculosis and rifampicin resistance in Mycobacterium tuberculosis. J Med Microbiol 2000;49:271-8.

73. Suarez PG, Floyd K, Portocarrero J, Alarcon E, Rapiti E, Ramos G, et al. Feasibility and costeffectiveness of standardised second-line drug treatment for chronic tuberculosis patients: a national cohort study in Peru. Lancet 2002; 359:1980-9.

74. Sterling TR, Lehmann HP, Frieden TR. Impact of DOTS compared with DOTS-plus on multidrug resistant tuberculosis and tuberculosis deaths: decision analysis. BMJ 2003;326:574.

75. Sharpe JN, Shively EH, Polk HCJ. Clinical and economic outcomes of oral linezolid versus intravenous vancomycin in the treatment of MRSA-complicated, lower-extremity skin and softtissue infections caused by methicillin-resistant Staphylococcus aureus. Am J Surg 2005;189:425-8.

76. Department of Health. Specialist Advisory Committee on Antimicrobial Resistance (SACAR). URL: http://www.advisorybodies.doh.gov.uk/sacar/ index.htm. Accessed 7 November 2005.

77. Struelens MJ. The epidemiology of antimicrobial resistance in hospital acquired infections: problems and possible solutions. BMJ 1998; 317:652-4.

78. NHS Centre for Reviews and Dissemination. Antimicrobial prophylaxis in colorectal surgery. Effective Health Care 1998;4:1-8.

79. De Gelder L, Ponciano JM, Abdo Z, Joyce P, Forney LJ, Top EM. Combining mathematical models and statistical methods to understand and predict the dynamics of antibiotic-sensitive mutants in a population of resistant bacteria during experimental evolution. Genetics 2004;168: 1131-44.

80. Lipsitch M, Bergstrom CT. Modeling of antibiotic resistance in the ICU - US slant. In: Weinstein RA, Bonten $\mathrm{M}$, editors. Infection control in the ICU environment. Boston, MA: Kluwer; 2002. chapt. 19.
81. Sebille V, Chevret S, Valleron A. Modeling the spread of resistant nosocomial pathogens in an intensive-care unit. Infect Control Hosp Epidemiol 1997;18:84-92.

82. Khan K, Muennig P, Behta M, Zivin JG. Global drug-resistance patterns and the management of latent tuberculosis infection in immigrants to the United States. N Engl J Med 2002;347:1850-9.

83. Mahamat A, Lavigne JP, Fabbro-Peray P, Kinowski JM, Daures JP, Sotto A. Evolution of fluoroquinolone resistance among Escherichia coli urinary tract isolates from a French university hospital: application of the dynamic regression model. Clin Microbiol Infect 2005;11:301-06.

84. Lopez-Lozano JM, Monnet DL, Yague A, Burgos A, Gonzalo N, Campillos P, et al. Modelling and forecasting antimicrobial resistance and its dynamic relationship to antimicrobial use: a time series analysis. Int J Antimicrob Agents 2000; 14:21-31.

85. Spiegelhalter DJ, Abrams KR, Myles JP. Bayesian approaches to clinical trials and health-care evaluation. Chichester: Wiley; 2004.

86. Briggs AH, Goeree R, Blackhouse G, O'Brien BJ. Probabilistic analysis of cost-effectiveness models: choosing between treatment strategies for gastroesophageal reflux disease. Med Decis Making 2002;22:290-308.

87. Claxton K, Posnett J. An economic approach to clinical trial design and research priority-setting. Health Econ 1996;5:513-24.

88. Claxton K, Sculpher M, Drummond M. A rational framework for decision making by the National Institute for Clinical Excellence (NICE). Lancet 2002;360:711-15.

89. Coast J, Smith RD. Antimicrobial resistance: cost and containment. Expert Rev Antiinfect Ther $2003 ; 1: 241-51$.

90. Ades AE, Sculpher M, Sutton A, Abrams K, Cooper N, Welton N, et al. Bayesian methods for evidence synthesis in cost-effectiveness analysis. Pharmacoeconomics 2006;24:1-19.

91. Brisson M, Edmunds WJ. Economic evaluation of vaccination programs: the impact of herdimmunity. Med Decis Making 2003;23:76-82.

92. Edmunds WJ, Medley GF, Nokes DJ. Evaluating the cost-effectiveness of vaccination programmes: a dynamic perspective. Stat Med 1999;18:3263-82.

93. Smith RD, Yago M, Millar M, Coast J. Assessing the macroeconomic impact of a healthcare problem: the application of computable general equilibrium analysis to antimicrobial resistance. J Health Econ 2005;24:1055-75.

94. Rudholm N. Economic implications of antibiotic resistance in a global economy. J Health Econ 2002;21:1071-83. 
95. Palmer S, Smith PC. Incorporating option values into the economic evaluation of health care technologies.J Health Econ 2000;19:755-66.

96. Rahman MH, Anson J. Peri-operative antibacterial prophylaxis. Pharm J 2004;272:743-5.

97. British Orthopaedic Association. Total hip replacement: a guide to best practice. London: British Orthopaedic Association; 1999.

98. Department of Health. Bacteraemia surveillance scheme - MRSA bacteraemia by NHS Trust: table 2 data for each 6 months, 2001-2005. URL:

http://www.dh.gov.uk/PublicationsAndStatistics/ Publications/PublicationsStatistics/Publications StatisticsArticle/fs/en?CONTENT_ID=4085951\& chk $=$ HBt2QD. Accessed 7 November 2005.

99. Ridgeway S, Wilson J, Charlet A, Kafatos G, Pearson A, Coello R. Infection of the surgical site after arthroplasty of the hip. J Bone Joint Surg Br 2005;87:844-50.

100. Cooke EM, Coello R, Sedgwick J, Ward V, Wilson J, Charlett A, et al. A national surveillance scheme for hospital associated infections in England. Team of the Nosocomial Infection National Surveillance Scheme.J Hosp Infect 2000;46:1-3.

101. Mohanty SS, Kay PR. Infection in total joint replacements. Why we screen MRSA when MRSE is the problem? J Bone Joint Surg Br 2004;86: 266-8.

102. Niederman MS. Impact of antibiotic resistance on clinical outcomes and the cost of care. Crit Care Med 2001;29 Suppl 4:N114-20.

103. Cosgrove SE, Qi Y, Kaye KS, Harbarth S, Karchmer AW, Carmeli Y. The impact of methicillin resistance in Staphylococcus aureus bacteremia on patient outcomes: mortality, length of stay, and hospital charges. Infect Control Hosp Epidemiol 2005;26:166-74.

104. Kunori T, Cookson B, Roberts JA, Stone S, Kibbler C. Cost-effectiveness of different MRSA screening methods. J Hosp Infect 2002;51:189-200.

105. Whitehouse JD, Friedman ND, Kirkland KB, Richardson WJ, Sexton DJ. The impact of surgicalsite infections following orthopedic surgery at a community hospital and a university hospital: adverse quality of life, excess length of stay, and extra cost. Infect Control Hosp Epidemiol 2002; 23:183-9.

106. Tengs TO, Wallace A. One thousand health-related quality-of-life estimates. Med Care 2000; 38:583-637.

107. Blom AW, Taylor AH, Pattison G, Whitehouse S, Bannister GC. Infection after total hip arthroplasty. The Avon experience. J Bone Joint Surg Br 2003;85:956-9.
108. Blom AW, Brown J, Taylor AH, Pattison G, Whitehouse S, Bannister GC. Infection after total knee arthroplasty. J Bone Joint Surg Br 2004; 86:688-91.

109. Lodise Jr TP, McKinnon PS, Rybak M. Prediction model to identify patients with staphylococcus aureus bacteremia at risk for methicillin resistance. Infect Control Hosp Epidemiol 2003;24:655-61.

110. Glenny A, Song F. Antimicrobial prophylaxis in total hip replacement: a systematic review. Health Technol Assess 1999;3(21).

111. Woodford N. Biological counterstrike: antibiotic resistance mechanisms of Gram-positive cocci. Clin Microbiol Infect 2005;11 Suppl 3:2-21.

112. Akins RL, Haase KK. Gram-positive resistance: pathogens, implications, and treatment options: insights from the Society of Infectious Diseases Pharmacists. Pharmacotherapy 2005;25:1001-10.

113. Antrum R, Gorst K, Galvin K, Thornton C, Wynne K, Malata C, et al. A comparison of teicoplanin vs. cephradine and metronidazole in surgical prophylaxis: an interim analysis. Scand J Infect Dis Suppl 1990;72:45-53.

114. Antrum RM, Galvin K, Gorst K, Thornton CA, Lansdown M, Kester RC. Teicoplanin vs cephradine and metronidazole in the prophylaxis of sepsis following vascular surgery: an interim analysis of an ongoing trial. Eur J Surg Suppl 1992;567:43-6.

115. Barlas S, Onursal E, Barlas C, Dayioglu E, Sismanoglu M, Tireli E, et al. Prophylaxis against infection in open cardiac operations. Med Bull Istanbul Med Fac 1993;26:51-6.

116. Bayston R, Bannister C, Boston V, Burman R, Burns B, Cooke F, et al. A prospective randomised controlled trial of antimicrobial prophylaxis in hydrocephalus shunt surgery. Z Kinderchir 1990;45 Suppl 1:5-7.

117. Bell SM, Gatus BJ, Shepherd BD. Antibioticprophylaxis for the prevention of late infections of prosthetic joints. Aust N Z J Surg 1990;60:177-81.

118. Brooks JC, Hatton RC, Winner L. Outcome analysis of antimicrobial prophylaxis in cardiothoracic surgery patients receiving cefazolin alone or a ceftazidime-vancomycin combination. Hosp Pharm 2002;37:380-5.

119. Bucknell SJ, Mohajeri M, Low J, McDonald M, Hill DG. Single-versus multiple-dose antibiotics prophylaxis for cardiac surgery. Aust N Z J Surg 2000;70:409-11.

120. Cone LA, Slavin KV. Intraoperative antibiotic prophylaxis in clean spinal surgery: a retrospective analysis in a consecutive series of 973 cases. Surg Neurol 2004;61:129-35.

121. Mastronardi L, Tatta C. Intraoperative antibiotic prophylaxis in clean spinal surgery: a retrospective 
analysis in a consecutive series of 973 cases. Surg Neurol 2004;61:129-35.

122. Dazzi C, Licheri S, Sias F, Secci L, Daniele GM. [Teicoplanin in the prevention of wound infections in major ambulatory surgery]. Ann Ital Chir 1994;65:121-3; in Italian.

123. de Lalla F, Viola R, Pellizzer G, Lazzarini L, Tramarin A, Fabris P. Regional prophylaxis with teicoplanin in monolateral or bilateral total knee replacement: an open study. Antimicrob Agents Chemother 2000;44:316-19.

124. de Lalla F. Antibiotic prophylaxis in orthopedic prosthetic surgery. J Chemother 2001;13:48-53.

125. De Lucas-Villarrubia JC, Lopez-Franco M, Granizo JJ, De Lucas-Garcia JC, Gomez-Barrena E. Strategy to control methicillin-resistant Staphylococcus aureus post-operative infection in orthopaedic surgery. Int Orthop 2004;28:16-20.

126. Exner K, Lang E, Borsche A, Lemperle G. Efficacy, tolerability and pharmacokinetics of teicoplanin in patients undergoing breast surgery. Eur J Surg Suppl 1992;567:33-8.

127. Ferro JF, de-Pablos M, Logrono MJ, Guisasola L, Aizpuru F. Postoperative contamination after using vancomycin and gentamicin during phacoemulsification. Arch Ophthalmol 1997; 115: $165-70$.

128. Feys J, Salvanet-Bouccara A, Emond JP, Dublanchet A. Vancomycin prophylaxis and intraocular contamination during cataract surgery. J Cataract Refract Surg 1997;23:894-7.

129. Fontanesi G, Costa P, Giancecchi F, Tartaglia I, Rotini R. Antibiotic and antithromboembolic prophylaxis in prosthetic surgery of the knee. Chir Organi Mov 1991;76:133-43.

130. Franchelli S, Leone MS, Rainero ML, Gipponi M, Cafiero F, Capuzzo R, et al. Antibiotic prophylaxis with teicoplanin in patients undergoing breast reconstruction with the transverse rectus abdominis myocutaneous flap. Eur J Plast Surg 1993;16:204-7.

131. Friberg D, Lundberg C. Antibiotic-prophylaxis in major head and neck-surgery when cleancontaminated wounds are established. Scand J Infect Dis 1990;70:87-90.

132. Gadallah MF, Ramdeen G, Torres C, Mignone J, Patel D, Mitchell L, et al. Preoperative vancomycin prophylaxis for newly placed peritoneal dialysis catheters prevents post-operative peritonitis. $A d v$ Perit Dial 2000;16:199-203.

133. Haines SJ. Ceftizoxime versus vancomycin and gentamicin in neurosurgical prophylaxis: a randomized, prospective, blinded clinical study [letter]. Neurosurgery 1993;33:949.

134. Isringhaus H, Lang E, Witte PU, Rogee P. Tolerability and pharmacokinetics of teicoplanin in patients undergoing heart surgery: preliminary results. Int J Antimicrob Agents 1992;1 Suppl 1: S31-6.

135. Karran SJ, Karran SE, Toyn K, Brough P. Antibiotic prophylaxis in clean surgical cases and the role of community surveillance. Eur J Surg Suppl 1992;567:31-2.

136. Lazzarini L, Pellizzer G, Stecca C, Viola R, de Lalla F. Postoperative infections following total knee replacement: an epidemiological study. J Chemother 2001;13:182-7.

137. Lazzarini L, Novelli A, Marzano N, Timillero L, Fallani S, Viola R, et al. Regional and systemic prophylaxis with teicoplanin in total knee arthroplasty: a tissue penetration study. J Arthroplasty 2003;18:342-6.

138. Mendivil Soto A, Mendivil MP. The effect of topical povidone-iodine, intraocular vancomycin, or both on aqueous humor cultures at the time of cataract surgery. Am J Ophthalmol 2001;131: 293-300.

139. Mini E, Nobili S, Periti P. Does surgical prophylaxis with teicoplanin constitute a therapeutic advance? J Chemother 1999;12 Suppl $5: 40-55$.

140. Nehrer S, Thalhammer F, Schwameis E, Breyer S, Kotz R. Teicoplanin in the prevention of infection in total hip replacement. Arch Orthop Trauma Surg 1998;118:32-6.

141. Niederhäuser U, Vogt M, Vogt P, Genoni M, Künzli A, Turina MI. Cardiac surgery in a highrisk group of patients: is prolonged postoperative antibiotic prophylaxis effective? J Thorac Cardiovasc Surg 1997;114:162-8.

142. Pear SM, Goldsmith DL, Williamson TH, Mandel D, Sethi GK, Arzouman DA, et al. Identification and voluntary reduction of vancomycin use for perioperative antibiotic prophylaxis during coronary artery bypass graft surgery. Infect Control Hosp Epidemiol 1998; 19:513-5.

143. Periti P, Stringa G, Donati L, Mazzei T, Mini E, Novelli A. Teicoplanin - its role as systemic therapy of burn infections and as prophylaxis for orthopaedic surgery. Eur J Surg Suppl 1992; 567:3-8.

144. Rao GG, Osman M, Johnson L, Ramsey D, Jones S, Fidler H. Prevention of percutaneous endoscopic gastrostomy site infections caused by methicillin-resistant Staphylococcus aureus.J Hosp Infect 2004;58:81-3.

145. Renz CL, Thurn JD, Finn HA, Lynch JP, Moss J. Antihistamine prophylaxis permits rapid vancomycin infusion. Crit Care Med 1999; 27:1732-7.

146. Saginur R, Bergeron MG. Prophylaxis of infectious complications in patients undergoing elective 
CABG and/or valve replacement surgery: teicoplanin vs cefazolin. Can J Infect Dis 1995;6 Suppl C:210.

147. Santini C, Baiocchi P, Serra P. Perioperative antibiotic prophylaxis in vascular surgery. Eur J Vasc Endovasc Surg 1997;14 Suppl A:13-14.

148. Sassone D, Germano P, Tagliabue P. [Teicoplanine in the prophylaxis of Gram + bacterial infections in immunodeficient patients after surgical postmastectomy reconstructive operations]. Riv It Chir Plast 1991;23:317-20; in Italian.

149. Seppala H, Al-Juhaish M, Jarvinen H, Laitinen R, Huovinen P. Effect of prophylactic antibiotics on antimicrobial resistance of viridans streptococci in the normal flora of cataract surgery patients. J Cataract Refract Surg 2004;30:307-15.

150. Shimada M, Saitoh A, Kano T. Oral administration of vancomycin in preventing postoperative methicillin-resistant Staphylococcus aureus enterocolitis. Clin Drug Invest 1995;10:12-16.

151. Skinner GRB, Ahmad A, Zafar A, Hullin MG, Dick D. Prophylaxis against staphylococcal infection before tertiary knee joint replacement. Orthopedics 2001;24:289-91.

152. Sobaci G, Tuncer K, Tas A, Ozyurt M, Bayer A, Kutlu U. The effect of intraoperative antibiotics in irrigating solutions on aqueous humor contamination and endophthalmitis after phacoemulsification surgery. Eur J Ophthalmol 2003;13:773-8.

153. Steer JA, Papini RPG, Wilson APR, McGrouther DA, Nakhla LS, Parkhouse N. Randomized placebo-controlled trial of teicoplanin in the antibiotic prophylaxis of infection following manipulation of burn wounds. Br J Surg 1997;84:848-53.

154. Tinelli M, Caprioglio S, Fanello M, Parisi A, Murone MV, Maffezzini E. [Study of a casuistry of infections in the Department of Vascular Surgery in the St. Matthew of Pavia Polyclinic during the years 1991-1993: perioperative antibiotic prophylaxis with vancomycin]. G Mal Infett Parassit 1994;46:950-53; in Italian.

155. Tinelli M, Fanello M, Murone MV, Maffezzini E. Epidemiological analysis of infections in a vascular surgery department in the years 1991 to 1994 perioperative vancomycin prophylaxis in prosthetic substitutions. Can J Infect Dis 1995;6 Suppl C:2012.

156. Trenholme GM, Delaria GA, Reichardt LE, Koenig GI, Parkhurst G, Segreti J, et al. Antibiotic prophylaxis for cardiovascular surgery: a comparison of vancomycin and cefuroxime. In Interscience Conference on Antimicrobial Agents and Chemotherapy. Program and Abstracts of the Thirty-first Interscience Conference on Antimicrobial Agents and Chemotherapy; 1991 Sept 29-Oct 2; Chicago, Illinois. Washington, DC: American Society for Microbiology; 1991. p. 311.

157. Wilson AP, Sturridge MF, Treasure T. Aminoglycoside toxicity following antibiotic prophylaxis in cardiac surgery. J Antimicrob Chemother 1990;26:713-20.

158. Zibari GB, Gadallah MF, Landreneau M, McMillan R, Bridges RM, Costley K, et al. Preoperative vancomycin prophylaxis decreases incidence of postoperative hemodialysis vascular access infections. Am J Kidney Dis 1997; 30:343-8. 



\section{Appendix I \\ Search strategy}

$\mathrm{T}$ his appendix presents the detailed searches carried out to inform the review and the economic model.

\section{Effectiveness review}

The core search strategy used for this review was as follows:

1. Vancomycin/

2. 1404-90-6.rn.

3. vancomycin.ti,ab.

4. vancocin.ti,ab.

5. lyphocin.ti,ab.

6. vancoled.ti,ab.

7. vancor.ti,ab.

8. Teicoplanin/

9. 61036-62-2.rn.

10. teicoplanin.ti,ab.

11. teichomycin\$.ti,ab.

12. targocid.ti,ab.

13. exp Glycopeptides/

14. Antibiotics, Glycopeptide/

15. glycopeptide $\$$.ti,ab.

16. Antibiotic Prophylaxis/

17. Premedication/

18. (antibiotic $\$$ adj2 (prophyla $\$$ or premedicat $\$$ or pre-medicat $\$$ or therapeutic $\$)$ ).ti,ab.

19. ((anti-microbial\$ or antimicrobial\$) adj2 (prophyla $\$$ or premedicat $\$$ or pre-medicat $\$$ or therapeutic\$)).ti,ab.

20. ((anti-bacterial $\$$ or antibacterial $\$)$ adj2 (prophyla $\$$ or premedicat $\$$ or pre-medicat $\$$ or therapeutic\$)).ti,ab.

21. ((anti-mycobacterial\$ or antimycobacterial $\$$ ) adj2 (prophyla $\$$ or premedicat $\$$ or premedicat\$ or therapeutic $\$)$ ).ti,ab.

22. ((bacteriocidal or bacteriocide $\$$ ) adj2 (prophyla $\$$ or premedicat $\$$ or pre-medicat $\$$ or therapeutic $\$)$ ).ti,ab.

23. or/1-22

24. Bacterial Infections/

25. ((bacteri $\$$ or wound $\$$ or tissue $\$$ or prosthe $\$$ ) adj2 (infect\$ or contam\$)).ti,ab.

26. soft tissue infections/

27. prosthesis-related infections/

28. Sepsis/

29. sepsis.ti,ab.

30. (hospital\$ adj2 infect\$).ti,ab.
31. (mrsa or VISA or GISA or VRSA).ti,ab.

32. ((methicillin $\$$ or meticillin $\$$ or methycillin $\$)$ adj resist\$).ti,ab.

33. Staphylococcaceae/

34. staphylococcus/

35. staphylococcus aureus/

36. staphylococcus epidermidis/

37. staphylococcus haemolyticus/

38. staphylococcus hominis/

39. staphylococc $\$ . t i, a b$.

40. micrococcus pyogenes.ti,ab.

41. exp Staphylococcal Infections/

42. Gram-Positive Bacteria/

43. gram-positive bacterial infections/

44. gram-positive bacteri\$.ti,ab.

45. Gram-Positive Cocci/

46. gram-positive cocci.ti,ab.

47. Methicillin Resistance/

48. Methicillin/

49. Penicillin Resistance/

50. Drug Resistance, Microbial/

51. or $/ 24-50$

52. exp Surgical Procedures, Operative/

53. (surgery or surgical or operat\$).ti,ab.

54. (preoperat $\$$ or pre-operat $\$$ ).ti,ab.

55. (intraoperat\$ or intra-operat\$).ti,ab.

56. (perioperat $\$$ or peri-operat\$).ti,ab.

57. exp "Prostheses and Implants"/

58. (prosthe\$ or implant\$).ti,ab.

59. (bypass or graft\$).ti,ab.

60. (resection or dissect $\$$ or incision $\$$ ).ti,ab.

61. (biopsy or biopsies).ti,ab.

62. amputat\$.ti,ab.

63. ((hip $\$$ or knee $\$$ or joint $\$$ ) adj (replac $\$$ or arthroplast\$)).ti,ab.

64. (c-section $\$$ or caesarean $\$$ or ceasarean $\$$ or cesarean $\$$ or caesarian $\$$ or ceasarian $\$$ or cesarian\$).ti,ab.

65. hysterectom $\$ . t i, a b$.

66. abortion $\$ . t i, a b$.

67. or $/ 52-66$

68. Surgical Wound Infection/

69. ((surgery or surgical) adj2 (infect $\$$ or contamin \$)).ti,ab.

70. ((postoperat $\$$ or post-operat $\$)$ adj2 infect\$).ti,ab.

71. (incision\$ adj2 (infect\$ or contam\$)).ti,ab.

72. or $/ 68-71$

73. 23 and 51 and 67

74. 23 and 72 
75. 73 or 74

76. limit 75 to $y r=" 1990-2005 "$

77. Animals/

78. Humans/

79. 77 not (77 and 78)

80. 76 not 79

81. (editorial or historical article or letter).pt.

82. 80 not 81

This strategy was designed for searching MEDLINE through the Ovid interface and was adapted as appropriate for all other databases searched, taking into account differences in indexing terms and search syntax for each database.

Full details of all databases searched and search strategies are provided below.

\section{MEDLINE: Ovid (http://gateway.ovid.com/athens)}

The MEDLINE search covered the date range 1990 to September 2005 (Week 1). The search was carried out on 19 September 2005 and identified 4062 records.

1. VANCOMYCIN/ (6227)

2. 1404-90-6.rn. (6227)

3. vancomycin.ti,ab. (9106)

4. vancocin.ti,ab. (12)

5. lyphocin.ti,ab. (0)

6. vancoled.ti,ab. (6)

7. vancor.ti,ab. (0)

8. Teicoplanin/ (1292)

9. 61036-62-2.rn. (1292)

10. teicoplanin.ti,ab. (1833)

11. teichomycin\$.ti,ab. (22)

12. targocid.ti,ab. (3)

13. exp Glycopeptides/ (35378)

14. Antibiotics, Glycopeptide/ (2650)

15. glycopeptide\$.ti,ab. (5862)

16. Antibiotic Prophylaxis/ (3945)

17. Premedication/ (9969)

18. (antibiotic $\$$ adj2 (prophyla $\$$ or premedicat $\$$ or pre-medicat $\$$ or therapeutic $\$)$ ).ti,ab. (7968)

19. ((anti-microbial\$ or antimicrobial\$) adj2 (prophyla\$ or premedicat\$ or pre-medicat\$ or therapeutic\$)).ti,ab. (1496)

20. ((anti-bacterial\$ or antibacterial\$) adj2 (prophyla $\$$ or premedicat\$ or pre-medicat $\$$ or therapeutic $\$)$ ).ti,ab. (319)

21. ((anti-mycobacterial\$ or antimycobacterial $\$$ ) adj2 (prophyla $\$$ or premedicat $\$$ or premedicat $\$$ or therapeutic $\$)$ ).ti,ab. (11)

22. ((bacteriocidal or bacteriocide $\$$ ) adj2 (prophyla $\$$ or premedicat\$ or pre-medicat $\$$ or therapeutic\$)).ti,ab. (0)
23. or/1-22 (61524)

24. Bacterial Infections/ (46266)

25. ((bacteri $\$$ or wound $\$$ or tissue $\$$ or prosthe $\$$ ) adj2 (infect\$ or contam $\$$ )).ti,ab. (50156)

26. soft tissue infections/ (858)

27. prosthesis-related infections/ (3291)

28. Sepsis/ (7383)

29. sepsis.ti,ab. (34192)

30. (hospital\$ adj2 infect\$).ti,ab. (7731)

31. (mrsa or VISA or GISA or VRSA).ti,ab. (4478)

32. ((methicillin $\$$ or meticillin $\$$ or methycillin $\$)$ adj resist\$).ti,ab. (7145)

33. Staphylococcaceae/ (39)

34. staphylococcus/ (18264)

35. staphylococcus aureus/ (27303)

36. staphylococcus epidermidis/ (3496)

37. staphylococcus haemolyticus/ (25)

38. staphylococcus hominis/ (9)

39. staphylococc $\$ . t i, a b .(60096)$

40. micrococcus pyogenes.ti,ab. (12)

41. exp Staphylococcal Infections/ (31180)

42. Gram-Positive Bacteria/ (5922)

43. gram-positive bacterial infections/ (3947)

44. gram-positive bacteri\$.ti,ab. (6983)

45. Gram-Positive Cocci/ (809)

46. gram-positive cocci.ti,ab. (2222)

47. Methicillin Resistance/ (5223)

48. Methicillin/ (2609)

49. Penicillin Resistance/ (9324)

50. Drug Resistance, Microbial/ (49349)

51. or/24-50 (257344)

52. exp Surgical Procedures, Operative/ (1436152)

53. (surgery or surgical or operat\$).ti,ab. (926821)

54. (preoperat\$ or pre-operat\$).ti,ab. (110649)

55. (intraoperat\$ or intra-operat\$).ti,ab. (46051)

56. (perioperat\$ or peri-operat\$).ti,ab. (26339)

57. exp "Prostheses and Implants"/ (222018)

58. (prosthe\$ or implant\$).ti,ab. (189473)

59. (bypass or graft\$).ti,ab. (174427)

60 . (resection or dissect\$ or incision $\$$ ).ti,ab. (177550)

61. (biopsy or biopsies).ti,ab. (168204)

62. amputat\$.ti,ab. (17334)

63. ((hip\$ or knee\$ or joint\$) adj (replac\$ or arthroplast\$)).ti,ab. (20854)

64. (c-section $\$$ or caesarean $\$$ or ceasarean $\$$ or cesarean $\$$ or caesarian $\$$ or ceasarian $\$$ or cesarian\$).ti,ab. (23578)

65. hysterectom $\$ . t i, a b .(14956)$

66. abortion \$.ti,ab. (30146)

67. or/52-66 (2282138)

68. Surgical Wound Infection/ (19970)

69. ((surgery or surgical) adj2 (infect\$ or contamin $\$)) . t i, a b .(6627)$

70. ((postoperat $\$$ or post-operat $\$)$ adj2 infect\$).ti,ab. (5381) 
71. (incision\$ adj2 (infect\$ or contam\$)).ti,ab. (308)

72. or/68-71 (26955)

73. 23 and 51 and 67 (5475)

74. 23 and $72(4201)$

75. 73 or $74(7222)$

76. limit 75 to $\mathrm{yr}=" 1990-2005 "$ (4432)

77. Animals/ (3795171)

78. Humans/ (8961704)

79. 77 not (77 and 78) (2905784)

80. 76 not $79(4257)$

81. (editorial or historical article or letter).pt. (924445)

82. 80 not 81 (4062)

\section{EMBASE: Ovid}

\section{(http://gateway.ovid.com/athens)}

The EMBASE search covered the date range 1990 to 2005 (Week 37). The search was carried out on 19 September 2005 and identified 6580 records.

1. VANCOMYCIN/ (26284)

2. 1404-90-6.rn. (26378)

3. vancomycin.ti,ab. (9224)

4. vancocin.ti,ab. (15)

5. lyphocin.ti,ab. (0)

6. vancoled.ti,ab. (6)

7. vancor.ti,ab. (0)

8. Teicoplanin/ (5029)

9. 61036-62-2.rn. (5054)

10. teicoplanin.ti,ab. (2030)

11. teichomycin \$.ti,ab. (25)

12. targocid.ti,ab. (4)

13. Glycopeptide/ (3062)

14. exp polypeptide antibiotic agent/ (50141)

15. glycopeptide $\$ . t i, a b .(4757)$

16. Antibiotic Prophylaxis/ (8434)

17. Premedication/ (5500)

18. (antibiotic $\$$ adj2 (prophyla $\$$ or premedicat $\$$ or pre-medicat\$ or therapeutic\$)).ti,ab. (7060)

19. ((anti-microbial\$ or antimicrobial\$) adj2 (prophyla $\$$ or premedicat\$ or pre-medicat $\$$ or therapeutic\$)).ti,ab. (1416)

20. ((anti-bacterial $\$$ or antibacterial $\$)$ adj2 (prophyla $\$$ or premedicat $\$$ or pre-medicat $\$$ or therapeutic\$)).ti,ab. (279)

21. ((anti-mycobacterial\$ or antimycobacterial\$) adj2 (prophyla $\$$ or premedicat\$ or premedicat $\$$ or therapeutic\$)).ti,ab. (14)

22. ((bacteriocidal or bacteriocide $\$$ ) adj2 (prophyla $\$$ or premedicat\$ or pre-medicat\$ or therapeutic\$)).ti,ab. (1)

23. or/1-22 (71761)

24. Bacterial Infection/ (41468)

25. ((bacteri $\$$ or wound $\$$ or tissue $\$$ or prosthe $\$$ ) adj2 (infect\$ or contam\$)).ti,ab. (40607)

26. Soft Tissue Infection/ (2337)
27. Prosthesis Infection/ (644)

28. Sepsis/ (29228)

29. sepsis.ti,ab. (29674)

30. (hospital\$ adj2 infect\$).ti,ab. (6281)

31. (mrsa or VISA or GISA or VRSA).ti,ab. (4372)

32. ((methicillin\$ or meticillin\$ or methycillin\$) adj resist\$).ti,ab. (7088)

33. Staphylococcaceae/ (5)

34. exp staphylococcus/ (50535)

35. staphylococc $\$ . t i, a b . ~(45241)$

36. micrococcus pyogenes.ti,ab. (2)

37. Staphylococcus Infection/ (3901)

38. gram positive bacterium/ (12173)

39. gram-positive bacteri\$.ti,ab. (6246)

40. gram positive cocci/ (758)

41. gram-positive cocci.ti,ab. (1929)

42. antibiotic resistance/ (39148)

43. Meticillin/ (9160)

44. Penicillin Resistance/ (3059)

45. drug resistance/ (29683)

46. or/24-45 (234065)

47. exp surgery/ (1122365)

48. (surgery or surgical or operat\$).ti,ab. (720418)

49. (preoperat\$ or pre-operat\$).ti,ab. (93506)

50. (intraoperat $\$$ or intra-operat\$).ti,ab. (40440)

51. (perioperat\$ or peri-operat\$).ti,ab. (23970)

52. exp "prostheses and orthoses"/ (110394)

53. (prosthe\$ or implant\$).ti,ab. (138687)

54. (bypass or graft\$).ti,ab. (147283)

55. (resection or dissect\$ or incision\$).ti,ab. (144691)

56. (biopsy or biopsies).ti,ab. (140919)

57. amputat\$.ti,ab. (13015)

58. ((hip\$ or knee\$ or joint\$) adj (replac\$ or arthroplast\$)).ti,ab. (17540)

59. (c-section $\$$ or caesarean $\$$ or ceasarean $\$$ or cesarean $\$$ or caesarian $\$$ or ceasarian $\$$ or cesarian\$).ti,ab. (19256)

60. hysterectom $\$ . t i, a b .(12939)$

61. abortion\$.ti,ab. (16484)

62. or/47-61 (1681584)

63. Surgical Infection/ (2502)

64. ((surgery or surgical) adj2 (infect $\$$ or contamin\$)).ti,ab. (5608)

65. ((postoperat $\$$ or post-operat $\$$ ) adj2 infect\$).ti,ab. (4461)

66. (incision\$ adj2 (infect\$ or contam\$)).ti,ab. (236)

67. or/63-66 (11011)

68. 23 and 46 and 62 (7692)

69. 23 and 67 (2314)

70. 68 or $69(8520)$

71. limit 70 to $\mathrm{yr}=" 1990-2005 "$ (6925)

72. Animal/ (15773)

73. Human/ (5003512)

74. 72 not (72 and 73) (12793)

75. 71 not $74(6924)$ 
76. (editorial or letter).pt. (438750)

77. 75 not $76(6580)$

\section{CINAHL: Ovid}

\section{(http://gateway.ovid.com/athens)}

The CINAHL search covered the date range 1990 to September 2005 (Week 2). The search was carried out on 19 September 2005 and identified 600 records.

1. VANCOMYCIN/ (315)

2. vancomycin.ti,ab. (602)

3. vancocin.ti,ab. (1)

4. lyphocin.ti,ab. (0)

5. vancoled.ti,ab. (0)

6. vancor.ti,ab. (0)

7. teicoplanin.ti,ab. (28)

8. teichomycin\$.ti,ab. (0)

9. targocid.ti,ab. (1)

10. Antibiotics, Peptide/ (19)

11. glycopeptide $\$ . t i, a b .(40)$

12. Antibiotics/ (5226)

13. Antibiotic Prophylaxis/ (1108)

14. Premedication/ (240)

15. (antibiotic\$ adj2 (prophyla\$ or premedicat\$ or pre-medicat $\$$ or therapeutic\$)).ti,ab. (497)

16. ((anti-microbial\$ or antimicrobial\$) adj2 (prophyla $\$$ or premedicat $\$$ or pre-medicat $\$$ or therapeutic\$)).ti,ab. (90)

17. ((anti-bacterial\$ or antibacterial\$) adj2 (prophyla $\$$ or premedicat\$ or pre-medicat\$ or therapeutic\$)).ti,ab. (9)

18. ((anti-mycobacterial\$ or antimycobacterial\$) adj2 (prophyla $\$$ or premedicat\$ or premedicat\$ or therapeutic $\$)$ ).ti,ab. (0)

19. ((bacteriocidal or bacteriocide $\$$ ) adj2 (prophyla $\$$ or premedicat $\$$ or pre-medicat $\$$ or therapeutic $\$)) . t i, a b .(0)$

20. or/1-19 (7110)

21. Bacterial Infections/ (1593)

22. (bacteri $\$$ or wound $\$$ or tissue $\$$ or prosthe $\$$ ) adj2 (infect\$ or contam\$)).ti,ab. (2079)

23. soft tissue infections/ (86)

24. prosthesis-related infections/ (53)

25. Sepsis/ (1390)

26. sepsis.ti,ab. (1594)

27. (hospital\$ adj2 infect\$).ti,ab. (1355)

28. (mrsa or VISA or GISA or VRSA).ti,ab. (820)

29. ((methicillin\$ or meticillin\$ or methycillin\$) adj resist\$).ti,ab. (746)

30. staphylococcus/ (214)

31. staphylococcus aureus/ (705)

32. staphylococc $\$ . t i, a b .(1618)$

33. micrococcus pyogenes.ti,ab. (0)

34. exp Staphylococcal Infections/ (1664)

35. Gram-Positive Bacteria/ (100)

36. gram-positive bacterial infections/ (129)
37. gram-positive bacteri\$.ti,ab. (69)

38. gram-positive cocci.ti,ab. (67)

39. Methicillin Resistance/ (1142)

40. Methicillin/ (24)

41. Drug Resistance, Microbial/ (2696)

42. or/21-41 (11275)

43. exp Surgery, Operative/ (65532)

44. (surgery or surgical or operat\$).ti,ab. (43410)

45. (preoperat\$ or pre-operat\$).ti,ab. (3800)

46. (intraoperat\$ or intra-operat\$).ti,ab. (1382)

47. (perioperat\$ or peri-operat\$).ti,ab. (3042)

48. (prosthe $\$$ or implant $\$$ ).ti,ab. (6316)

49. (bypass or graft\$).ti,ab. (5358)

50. (resection or dissect\$ or incision\$).ti,ab. (3051)

51. (biopsy or biopsies).ti,ab. (2412)

52. amputat\$.ti,ab. (1638)

53. ((hip\$ or knee\$ or joint \$) adj (replac\$ or arthroplast\$)).ti,ab. (1656)

54. (c-section\$ or caesarean\$ or ceasarean $\$$ or cesarean $\$$ or caesarian $\$$ or ceasarian $\$$ or cesarian\$).ti,ab. (2043)

55. hysterectom $\$ . t i, a b .(815)$

56. abortion\$.ti,ab. (1374)

57. or/43-56 (96734)

58. Surgical Wound Infection/ (1374)

59. ((surgery or surgical) adj2 (infect\$ or contamin\$)).ti,ab. (718)

60. ((postoperat\$ or post-operat\$) adj2 infect\$).ti,ab. (256)

61. (incision\$ adj2 (infect\$ or contam\$)).ti,ab. (20)

62. or/58-61 (1766)

63. 20 and 42 and 57 (432)

64. 20 and 62 (345)

65. 63 or $64(642)$

66. limit 65 to $\mathrm{yr}=" 1990-2005 "$ (617)

67. (editorial or historical material or letter).pt. (88413)

68. 66 not $67(600)$

\section{CENTRAL: The Cochrane Library (CD-ROM issue 2005/3)}

Issue 2005/3 of the Cochrane Library was searched to identify trials on the Cochrane Central Register of Controlled Trials (CENTRAL). The search was carried out on 20 September 2005, with the date range set at 1990 to date. The search identified 1473 trials.

1. VANCOMYCIN single term (MeSH) (301)

2. vancomycin (587)

3. vancocin $(0)$

4. lyphocin (0)

5. vancoled (1)

6. vancor $(0)$

7. TEICOPLANIN single term (MeSH) (136) 
8. teicoplanin (207)

9. teichomycin* $(0)$

10. $\operatorname{targocid}(0)$

11. GLYCOPEPTIDES explode tree 1 (MeSH) (1027)

12. ANTIBIOTICS GLYCOPEPTIDE single term (MeSH) (123)

13. glycopeptide* (259)

14. ANTIBIOTIC PROPHYLAXIS single term (MeSH) (590)

15. PREMEDICATION single term $(\mathrm{MeSH})$ (2395)

16. ((antibiotic* near prophyla*) or (antibiotic* near premedicat*) or (antibiotic* near premedicat*) or (antibiotic* near therapeutic*)) (4428)

17. ((anti-microbial* near prophyla*) or (antimicrobial* near premedicat*) or (antimicrobial* near pre-medicat*) or (antimicrobial* near therapeutic*) or (antimicrobial* near prophyla*) or (antimicrobial* near premedicat*) or (antimicrobial* near pre-medicat*) or (antimicrobial* near therapeutic*)) (425)

18. ((anti-bacterial* near prophyla*) or (antibacterial* near premedicat*) or (antibacterial* near pre-medicat*) or (antibacterial* near therapeutic*) or (antibacterial* near prophyla*) or (antibacterial* near premedicat*) or (antibacterial* near premedicat*) or (antibacterial* near therapeutic*)) (2816)

19. ((anti-mycobacterial* near prophyla*) or (antimycobacterial* near premedicat*) or (antimycobacterial* near pre-medicat*) or (antimycobacterial* near therapeutic*) or (antimycobacterial* near prophyla*) or (antimycobacterial* near premedicat*) or (antimycobacterial* near pre-medicat*) or (antimycobacterial* near therapeutic*)) (0)

20. ((bacteriocidal near prophyla*) or (bacteriocidal near premedicat*) or (bacteriocidal near pre-medicat*) or (bacteriocidal near therapeutic*) or (bacteriocide* near prophyla*) or (bacteriocide* near premedicat*) or (bacteriocide* near pre-medicat*) or (bacteriocide* near therapeutic*)) (0)

21. (\#1 or \#2 or \#3 or \#4 or \#5 or \#6 or $\# 7$ or $\# 8$ or \#9 or \#10 or \#11 or \#12 or \#13 or $\# 14$ or \#15 or \#16 or \#17 or \#18 or \#19 or \#20) (9716)

22. BACTERIAL INFECTIONS single term (MeSH) (2448)

23. ((bacteri* near infect*) or (wound* near infect*) or (tissue* near infect*) or (prosthe* near infect*) or (bacteri* near contam*) or (wound* near contam*) or (tissue* near contam*) or (prosthe* near contam*)) (9624)

24. SOFT TISSUE INFECTIONS single term (MeSH) (29)

25. PROSTHESIS-RELATED INFECTIONS single term (MeSH) (73)

26. SEPSIS single term (MeSH) (392)

27. sepsis (2712)

28. (hospital* near infect*) (3306)

29. (mrsa or visa or gisa or vrsa) (145)

30. ((methicillin* next resist*) or (meticillin* next resist*) or (methycillin* next resist*)) (240)

31. STAPHYLOCOCCACEAE single term $(\mathrm{MeSH})(0)$

32. STAPHYLOCOCCUS explode tree $1(\mathrm{MeSH})$ (599)

33. staphylococc* (2248)

34. (micrococcus next pyogenes) $(0)$

35. STAPHYLOCOCCAL INFECTIONS explode tree $1(\mathrm{MeSH})(680)$

36. GRAM-POSITIVE BACTERIA single term (MeSH) (137)

37. GRAM-POSITIVE BACTERIAL INFECTIONS single term (MeSH) (143)

38. (gram-positive next bacteri*) (380)

39. GRAM-POSITIVE COCCI single term (MeSH) (9)

40. (gram-positive next cocci) (113)

41. METHICILLIN RESISTANCE single term $(\mathrm{MeSH})(110)$

42. METHICILLIN single term (MeSH) (40)

43. PENICILLIN RESISTANCE single term (MeSH) (210)

44. DRUG RESISTANCE MICROBIAL single term $(\mathrm{MeSH})(768)$

45. (\#22 or \#23 or \#24 or \#25 or \#26 or \#27 or $\# 28$ or \#29 or \#30 or \#31 or \#32 or \#33 or $\# 34$ or \#35 or \#36 or \#37 or \#38 or \#39 or \#40 or \#41 or \#42 or \#43 or \#44) (14811)

46. SURGICAL PROCEDURES OPERATIVE explode tree 1 (MeSH) (50730)

47. (surgery or surgical or operat*) (74595)

48. (preoperat* or pre-operat*) (10166)

49. (intraoperat* or intra-operat*) (6531)

50. (perioperat* or peri-operat*) (4142)

51. PROSTHESES AND IMPLANTS explode tree 1 (MeSH) (6015)

52. (prosthe* or implant*) (9161)

53. (bypass or graft*) (12093)

54. (resection or dissect* or incision*) (7502)

55. (biopsy or biopsies) (7126)

56. amputat* (720)

57. ((hip* next replac*) or (knee* next replac*) or (joint* next replac*) or (arthroplast* next replac*) or (hip* next arthroplast*) or (knee* next arthroplast*) or (joint* next arthroplast*) or (arthroplast* next arthroplast*)) (2662) 
58. (c-section* or caesarean* or ceasarean* or cesarean* or caesarian* or ceasarian* or cesarian*) (3532)

59. hysterectom* (2159)

60. abortion* (1642)

61. (\#46 or \#47 or \#48 or \#49 or \#50 or \#51 or $\# 52$ or \#53 or \#54 or \#55 or \#56 or \#57 or \#58 or \#59 or \#60) (104735)

62. SURGICAL WOUND INFECTION single term (MeSH) (1921)

63. ((surgery near infect*) or (surgical near infect*) or (surgery near contamin*) or (surgical near contamin*)) (3528)

64. ((postoperat* near infect*) or (post-operat* near infect*)) (1942)

65. ((incision* near infect*) or (incision* near contam*)) (136)

66. (\#62 or \#63 or \#64 or \#65) (4312)

67. (\#21 and \#45 and \#61) (2270)

68. (\#21 and \#66) (1830)

69. (\#67 or \#68) (2489)

70. \#69 ( 1990 to current date ) (1473)

71. ANIMALS single term (MeSH) (4802)

72. HUMANS check tag (MeSH) (260279)

73. (\#71 and (not (\#71 and \#72))) (14)

74. (\#70 and (not \#73)) (1473)

\section{Science Citation Index: MIMAS Web of Science (http://wok.mimas.ac.uk)}

The Science Citation Index search covered the date range 1990 to date. The search was carried out on 20 September 2005 and identified 2656 records.

1. $\mathrm{TS}=$ (vancomycin or vancocin or lyphocin or vancoled or vancor or teicoplanin or teichomycin* or targocid or glycopeptide*) (13733)

2. $\mathrm{TS}=\left(\left(\right.\right.$ antibiotic $^{*}$ or anti-microbial ${ }^{*}$ or antimicrobial* or anti-bacterial* or antibacterial* or anti-mycobacterial* or antimycobacterial* or bacteriocidal or bacteriocide*) same (prophyla* or premedicat* or pre-medicat* or therapeutic*)) (7736)

3. \#2 OR \# 1 (20976)

4. $\mathrm{TS}=\left(\left(\right.\right.$ bacteri $^{*}$ or wound $*$ or tissue* or prosthe*) same (infect* or contam*)) (59288)

5. TS $=$ (sepsis or staphylococc* or micrococcus pyogenes or gram-positive bacteri* or grampositive cocci) (75140)

6. $\mathrm{TS}=($ hospital* same infect*) $(11841)$

7. $\mathrm{TS}=(\mathrm{mrsa}$ or visa or gisa or vrsa or methicillin* resist* or meticillin* resist* or methycillin* resist*) (6775)

8. \#7 OR \#6 OR \#5 OR \#4 (> 100000)
9. $\mathrm{TS}=($ surgery or surgical or operat* or preoperat* or pre-operat* or intraoperat* or intra-operat* or perioperat* or peri-operat*) $(>100000)$

10. $\mathrm{TS}=($ prosthe $*$ or implant $*$ or bypass or graft $*$ or resection or dissect* or incision* or biopsy or biopsies or amputat*) (>100000)

11. $\mathrm{TS}=($ hip* replac* or knee* replac* or joint* replac* or hip* arthroplast* or knee* arthroplast* or joint* arthroplast*) (16290)

12. $\mathrm{TS}=(\mathrm{c}$-section $*$ or caesarean* or ceasarean* or cesarean* or caesarian* or ceasarian* or cesarian* or hysterectom* or abortion*) (37505)

13. \#12 OR \#11 OR \#10 OR \#9 (>100000)

14. $\mathrm{TS}=(($ surgery or surgical or incision* $)$ same (infect* or contamin*)) (9696)

15. $\mathrm{TS}=(($ postoperat* or post-operat* $)$ same infect*) (4928)

16. \#15 OR \# 14 (13128)

17. \#13 AND \#8 AND \#3 (2293)

18. \#16 AND \#3 (1457)

19. \#18 OR \# 17 (2656)

\section{BIOSIS: Edina}

\section{(http://edina.ac.uk/biosis/index.shtml)}

The BIOSIS search covered the date range 1990 to date. The search was carried out on 21

September 2005 and identified 1516 records and 329 records for the first and second search, respectively. The results from the two searches were de-duplicated in an endnote library, resulting in 1714 unique records.

Two separate searches were run on BIOSIS as the 'or' operator was not working at the time of searching.

\section{First search in Title/Subjects/Abstract}

vancomycin or vancocin or lyphocin or vancoled or vancor or teicoplanin or teichomycin* or targocid or glycopeptide* or antibiotic* $\mathrm{n} 2$ prophyla* or antibiotic* $\mathrm{n} 2$ premedicat* or antibiotic* $\mathrm{n} 2$ pre-medicat* or antibiotic* $\mathrm{n} 2$ therapeutic* or anti-microbial* $\mathrm{n} 2$ prophyla* or anti-microbial* $\mathrm{n} 2$ premedicat* or anti-microbial* $\mathrm{n} 2$ pre-medicat* or anti-microbial* $\mathrm{n} 2$ therapeutic* or antimicrobial* $\mathrm{n} 2$ prophyla* or antimicrobial* $\mathrm{n} 2$ premedicat* or antimicrobial* n2 pre-medicat* or antimicrobial* $\mathrm{n} 2$ therapeutic* or anti-bacterial* $\mathrm{n} 2$ prophyla* or anti-bacterial* $\mathrm{n} 2$ premedicat* or anti-bacterial* $\mathrm{n} 2$ pre-medicat* or anti-bacterial* $\mathrm{n} 2$ therapeutic* or antibacterial* $\mathrm{n} 2$ prophyla* or antibacterial* $\mathrm{n} 2$ premedicat* or antibacterial* $\mathrm{n} 2$ pre-medicat* or antibacterial* $\mathrm{n} 2$ therapeutic* or anti-mycobacterial* $\mathrm{n} 2$ prophyla* or anti-mycobacterial* $\mathrm{n} 2$ premedicat* or anti- 
mycobacterial* n2 pre-medicat* or anti-

mycobacterial* $\mathrm{n} 2$ therapeutic* or

antimycobacterial* n2 prophyla* or

antimycobacterial* $\mathrm{n} 2$ premedicat* or

antimycobacterial* n2 pre-medicat* or

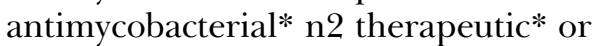

bacteriocidal $\mathrm{n} 2$ prophyla* or bacteriocidal $\mathrm{n} 2$ premedicat* or bacteriocidal $\mathrm{n} 2$ pre-medicat* or bacteriocidal $n 2$ therapeutic* or bacteriocide* $\mathrm{n} 2$ prophyla* or bacteriocide* $\mathrm{n} 2$ premedicat* or bacteriocide* $n 2$ pre-medicat* or bacteriocide* $\mathrm{n} 2$ therapeutic* (19300)

AND

bacteri* $\mathrm{n} 2$ infect or wound* $\mathrm{n} 2$ infect or tissue* $\mathrm{n} 2$ infect or prosthe* $\mathrm{n} 2$ infect* or bacteri* $\mathrm{n} 2$ contam* or wound* $\mathrm{n} 2$ contam* or tissue* $\mathrm{n} 2$ contam* or prosthe* $\mathrm{n} 2$ contam* or sepsis or staphylococc* or "micrococcus pyogenes" or grampositive w bacteri* or gram-positive w cocci or hospital* $n 2$ infect* or mrsa or visa or gisa or vrsa or methicillin* $\mathrm{w}$ resist* or meticillin* $\mathrm{w}$ resist* or methycillin* ${ }^{\mathrm{w}}$ resist* (124606)

AND

surgery or surgical or operat* or preoperat* or pre-operat* or intraoperat* or intra-operat* or perioperat* or peri-operat* or prosthe* or implant* or bypass or graft* or resection or dissect* or incision* or biopsy or biopsies or amputat* or hip* w replac* or knee* w replac* or joint* $\mathrm{w}$ replac* or hip* w arthroplast* or knee* ${ }^{*}$ arthroplast* or joint* w arthroplast* or c-section* or caesarean* or ceasarean* or cesarean* or caesarian* or ceasarian* or cesarian* or hysterectom* or abortion* (915038)

\section{Second search in Title/Subjects/Abstract}

vancomycin or vancocin or lyphocin or vancoled or vancor or teicoplanin or teichomycin* or targocid or glycopeptide* or antibiotic* $\mathrm{n} 2$ prophyla* or antibiotic* $\mathrm{n} 2$ premedicat* or antibiotic* $\mathrm{n} 2$ pre-medicat* or antibiotic* $\mathrm{n} 2$ therapeutic* or anti-microbial* n2 prophyla* or anti-microbial* $n 2$ premedicat* or anti-microbial* $\mathrm{n} 2$ pre-medicat* or anti-microbial* $\mathrm{n} 2$ therapeutic* or antimicrobial* n2 prophyla* or antimicrobial* $\mathrm{n} 2$ premedicat* or antimicrobial* n2 pre-medicat* or antimicrobial* $\mathrm{n} 2$ therapeutic* or anti-bacterial* n2 prophyla* or anti-bacterial* $\mathrm{n} 2$ premedicat* or anti-bacterial* $\mathrm{n} 2$ pre-medicat* or anti-bacterial* $\mathrm{n} 2$ therapeutic* or antibacterial* n2 prophyla* or antibacterial* n2 premedicat* or antibacterial* $\mathrm{n} 2$ pre-medicat* or antibacterial* $\mathrm{n} 2$ therapeutic* or anti-mycobacterial* n2 prophyla* or anti-mycobacterial* $\mathrm{n} 2$ premedicat* or antimycobacterial* $\mathrm{n} 2$ pre-medicat* or antimycobacterial* $\mathrm{n} 2$ therapeutic* or antimycobacterial* $\mathrm{n} 2$ prophyla* or antimycobacterial* $\mathrm{n} 2$ premedicat* or antimycobacterial* n2 pre-medicat* or antimycobacterial* $\mathrm{n} 2$ therapeutic* or bacteriocidal $\mathrm{n} 2$ prophyla* or bacteriocidal $\mathrm{n} 2$ premedicat* or bacteriocidal $\mathrm{n} 2$ pre-medicat* or bacteriocidal $\mathrm{n} 2$ therapeutic* or bacteriocide* $\mathrm{n} 2$ prophyla* or bacteriocide* $\mathrm{n} 2$ premedicat* or bacteriocide* $\mathrm{n} 2$ pre-medicat* or bacteriocide* $\mathrm{n} 2$ therapeutic* (19300)

AND

surgery $\mathrm{n} 2$ infect* or surgical $\mathrm{n} 2$ infect* or incision* $\mathrm{n} 2$ infect* or surgery $\mathrm{n} 2$ contamin*or surgical $n 2$ contamin* or incision* $n 2$ contamin* or postoperat* $\mathrm{n} 2$ infect* or post-operat* $\mathrm{n} 2$ infect* (1985)

\section{ISI Proceedings - Science and Technology edition: MIMAS Web of Science (http://wok.mimas.ac.uk)}

The ISI Proceedings search covered the date range 1990 to date. The search was carried out on 22 September 2005 and identified 324 records.

1. $\mathrm{TS}=$ (vancomycin or vancocin or lyphocin or vancoled or vancor or teicoplanin or teichomycin* or targocid or glycopeptide*) (1257)

2. $\mathrm{TS}=(($ antibiotic $*$ or anti-microbial* or antimicrobial* or anti-bacterial* or antibacterial* or anti-mycobacterial* or antimycobacterial* or bacteriocidal or bacteriocide*) same (prophyla* or premedicat* or pre-medicat* or therapeutic*)) (915)

3. \#2 OR \# 1 (2119)

4. $\mathrm{TS}=\left(\left(\right.\right.$ bacteri $^{*}$ or wound $*$ or tissue* or prosthe*) same (infect* or contam*)) (6558)

5. TS $=($ sepsis or staphylococc* or micrococcus pyogenes or gram-positive bacteri* or grampositive cocci) (8186)

6. TS $=($ hospital* same infect* $)(1405)$

7. $\mathrm{TS}=(\mathrm{mrsa}$ or visa or gisa or vrsa or methicillin* resist* or meticillin* resist* or methycillin* resist*) (767)

8. \#7 OR \#6 OR \#5 OR \#4 (14920)

9. $\mathrm{TS}=$ (surgery or surgical or operat* or preoperat* or pre-operat* or intraoperat* or intra-operat* or perioperat* or peri-operat*) $(>100000)$

10. TS $=($ prosthe* or implant* or bypass or graft* or resection or dissect* or incision* or biopsy or biopsies or amputat*) (88681)

11. $\mathrm{TS}=($ hip* replac* or knee* replac* or joint* replac* or hip* arthroplast* or knee* arthroplast* or joint* arthroplast*) (2216)

12. $\mathrm{TS}=(\mathrm{c}$-section* or caesarean* or ceasarean* or cesarean* or caesarian* or ceasarian* or 
cesarian* or hysterectom* or abortion*) (3823)

13. \#12 OR \# 11 OR \#10 OR \#9 (>100000)

14. $\mathrm{TS}=(($ surgery or surgical or incision* $*)$ same (infect* or contamin*)) (1329)

15. $\mathrm{TS}=\left(\left(\right.\right.$ postoperat* or post-operat $\left.{ }^{*}\right)$ same infect*) (715)

16. \#15 OR \# 14 (1826)

17. \#13 AND \#8 AND \#3 (277)

18. \#16 AND \#3 (196)

19. \#18 OR \#17 (324)

\section{Inside Conferences: Dialog (File 65)}

The Inside Conferences search covered the date range 1990 to date. The search was carried out on 24 November 2005 and identified 121 records.

Records on the Inside Conferences databases are very brief and abstracts are not included. The 'infection' terms in the strategy were removed for this search, to broaden it, so that potentially relevant papers might be identified.

1. s vancomycin or vancocin or lyphocin or vancoled or vancor or teicoplanin or teichomycin? or targocid or glycopeptide? (506)

2. s ((antibiotic? or anti(w)microbial? or antimicrobial? or anti(w)bacterial? or antibacterial? or anti(w)mycobacterial? or antimycobacterial? or bacteriocidal or bacteriocide?)(2n)(prophyla? or premedicat? or pre(w)medicat? or therapeutic?)) (199)

3. s surgery or surgical or operat? or preoperat? or pre(w)operat? or intraoperat? or intra(w)operat? or perioperat? or peri(w)operat? or prosthe? or implant? or bypass or graft? or resection or dissect? or incision? or biopsy or biopsies or amputat? or hysterectom? or abortion? (157507)

4. s ((hip or hips or knee or knees or joint?)(w)(replac? or arthroplast?)) (2974)

5. $\mathrm{s} \mathrm{c}(\mathrm{w})$ section? or caesarean? or ceasarean? or cesarean? or caesarian? or ceasarian? or cesarian? (593)

6. s s3:s5 (160417)

7. s s1 and s6 (22)

8. $\quad$ s s2 and s6 (99)

9. $\quad$ s s7 or s8 $(121)$

10. s s9/1990:2005 (121)

\section{National Research Register: Internet (http://www.update-software.com/ national)}

Issue 2005/3 of the National Research Register was searched to identify ongoing and recently carried out on 22 September 2005 and identified 76 projects.

1. VANCOMYCIN single term (MeSH) (26)

2. vancomycin $(61)$

3. $\operatorname{vancocin}(0)$

4. lyphocin $(0)$

5. vancoled $(0)$

6. vancor $(0)$

7. TEICOPLANIN single term (MeSH) (13)

8. teicoplanin (29)

9. teichomycin* $(0)$

10. $\operatorname{targocid}(0)$

11. GLYCOPEPTIDES explode tree $1(\mathrm{MeSH})$ (78)

12. ANTIBIOTICS GLYCOPEPTIDE single term (MeSH) (22)

13. glycopeptide* (39)

14. ANTIBIOTIC PROPHYLAXIS single term (MeSH) (46)

15. PREMEDICATION single term $(\mathrm{MeSH})(8)$

16. ((antibiotic* near prophyla*) or (antibiotic* near premedicat*) or (antibiotic* near (pre next medicat*)) or antibiotic* near therapeutic*)) (519)

17. (((anti next microbial*) near prophyla*) or ((anti next microbial*) near premedicat*) or ((anti next microbial*) near (pre next medicat*)) or ((anti next microbial*) near therapeutic*) or (antimicrobial* near prophyla*) or (antimicrobial* near premedicat*) or (antimicrobial* near (pre next

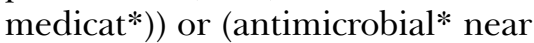
therapeutic*)) (16)

18. (((anti next bacterial*) near prophyla*) or ((anti next bacterial*) near premedicat*) or ((anti next bacterial*) near (pre next medicat*)) or ((anti next bacterial*) near therapeutic*) or (antibacterial* near prophyla*) or (antibacterial* near premedicat*) or (antibacterial* near (pre next medicat*)) or (antibacterial* near therapeutic*)) (32)

19. (((anti next mycobacterial*) near prophyla*) or ((anti next mycobacterial*) near premedicat*) or ((anti next mycobacterial*) near (pre next medicat*)) or ((anti next mycobacterial*) near therapeutic*) or (antimycobacterial* near prophyla*) or (antimycobacterial* near premedicat*) or (antimycobacterial* near (pre next medicat*)) or (antimycobacterial* near therapeutic*)) (0)

20. ((bacteriocidal near prophyla*) or (bacteriocidal near premedicat*) or (bacteriocidal near (pre next medicat*)) or (bacteriocidal near therapeutic*) or (bacteriocide* near prophyla*) or 
(bacteriocide* near premedicat*) or (bacteriocide* near (pre next medicat*)) or (bacteriocide* near therapeutic*)) (0)

21. (\#1 or \#2 or \#3 or \#4 or \#5 or \#6 or \#7 or \#8 or \#9 or \#10 or \#11 or \#12 or \#13 or \#14 or \#15 or \#16 or \#17 or \#18 or \#19 or \#20) (673)

22. BACTERIAL INFECTIONS single term (MeSH) (158)

23. ((bacteri* near infect*) or (wound* near infect*) or (tissue* near infect*) or (prosthe* near infect*) or (bacteri* near contam*) or (wound* near contam*) or (tissue* near contam*) or (prosthe* near contam*)) (926)

24. SOFT TISSUE INFECTIONS single term $(\mathrm{MeSH})(2)$

25. PROSTHESIS-RELATED INFECTIONS single term (MeSH) (12)

26. SEPSIS single term (MeSH) (194)

27. sepsis $(580)$

28. (hospital* near infect*) (1049)

29. (mrsa or visa or gisa or vrsa) (159)

30. ((methicillin* next resist*) or (meticillin* next resist*) or (methycillin* next resist*)) (126)

31. STAPHYLOCOCCACEAE single term $(\mathrm{MeSH})(0)$

32. STAPHYLOCOCCUS explode tree 1 (MeSH) (96)

33. staphylococc* $(241)$

34. (micrococcus next pyogenes) $(0)$

35. STAPHYLOCOCCAL INFECTIONS explode tree $1(\mathrm{MeSH})(119)$

36. GRAM-POSITIVE BACTERIA single term (MeSH) (5)

37. GRAM-POSITIVE BACTERIAL INFECTIONS single term (MeSH) (24)

38. (gram next positive next bacteri*) (36)

39. GRAM-POSITIVE COCCI single term $(\mathrm{MeSH})(1)$

40. (gram next positive next cocci) (6)

41. METHICILLIN RESISTANCE single term (MeSH) (88)

42. METHICILLIN single term (MeSH) (4)

43. PENICILLIN RESISTANCE single term $(\mathrm{MeSH})(0)$

44. DRUG RESISTANCE MICROBIAL single term (MeSH) (155)

45. (\#22 or \#23 or \#24 or \#25 or \#26 or \#27 or $\# 28$ or \#29 or \#30 or \#31 or \#32 or \#33 or \#34 or \#35 or \#36 or \#37 or \#38 or \#39 or \#40 or \#41 or \#42 or \#43 or \#44) (2734)

46. SURGICAL PROCEDURES OPERATIVE explode tree $1(\mathrm{MeSH})(8738)$

47. (surgery or surgical or operat*) (19869)

48. (preoperat* or (pre next operat*)) (1607)

49. (intraoperat* or (intra next operat*)) (483)

50. (perioperat* or (peri next operat*)) (468)
51. PROSTHESES AND IMPLANTS explode tree $1(\mathrm{MeSH})(1047)$

52. (prosthe* or implant*) (1924)

53. (bypass or graft*) (2140)

54. (resection or dissect* or incision*) (1720)

55. (biopsy or biopsies) (2457)

56. amputat* (335)

57. ((hip* next replac*) or (knee* next replac*) or (joint* next replac*) or (arthroplast* next replac*) or (hip* next arthroplast*) or (knee* next arthroplast*) or (joint* next arthroplast*) or (arthroplast* next arthroplast*)) (1046)

58. ((c next section*) or caesarean* or ceasarean* or cesarean* or caesarian* or ceasarian* or cesarian*) (540)

59. hysterectom* $(358)$

60. abortion* (298)

61. (\#46 or \#47 or \#48 or \#49 or \#50 or \#51 or $\# 52$ or \#53 or \#54 or \#55 or \#56 or \#57 or \#58 or \#59 or \#60) (616)

62. SURGICAL WOUND INFECTION single term (MeSH) (72)

63. ((surgery near infect*) or (surgical near infect*) or (surgery near contamin*) or (surgical near contamin*)) (260)

64. ((incision* near infect*) or (incision* near contam*)) (6)

65. ((postoperat* near infect*) or ((post next operat*) near infect*)) (171)

66. (\#62 or \#63 or \#64 or \#65) (366)

67. (\#21 and \#45 and \#61) (63)

68. (\#21 and \#66) (48)

69. (\#67 or \#68) (76)

\section{metaRegister of Controlled Trials: Current Controlled Trials (http://controlled-trials.com/mrct)}

The mRCT was searched on the Internet on 23 September 2005. The results were scanned for relevance and four potentially relevant trials were identified.

The search interface for the mRCT allows only very simple searching. The following terms were entered line-by-line:

- vancomycin OR vancocin OR lyphocin OR vancoled $\mathrm{OR}$ vancor $\mathrm{OR}$ teicoplanin $\mathrm{OR}$ teichomycin\% OR targocid OR glycopeptide\% (25)

- "antibiotic prophylaxis" OR "anti-microbial prophylaxis" OR "antimicrobial prophylaxis" OR "anti-bacterial prophylaxis" OR "antibacterial prophylaxis" (20)

- "surgical wound infection" OR "surgical wound infections" or "surgical infection" OR "surgical 
infections" OR "surgical contamination" OR

"surgical contaminations" (1)

- "postoperative wound infection" OR "postoperative wound infection" OR "postoperative wound infections" OR "post-operative wound infections" (1)

- "postoperative infection" OR "post-operative infection" OR "postoperative infections" OR "post-operative infections" (4).

\section{National Technical Information Service: Internet (http://www.ntis.gov/search/ index.asp? loc $=3-0-0$ )}

The NTIS was searched on the Internet on 23 September 2005. The results were scanned for relevance and one potentially relevant record was identified.

The search interface for the NTIS allows only very simple searching. The following terms were entered line-by-line:

- vancomycin OR vancocin OR lyphocin OR vancoled OR vancor OR teicoplanin OR teichomycin OR teichomycins OR targocid OR glycopeptide OR glycopeptides (30)

- "antibiotic prophylaxis" OR "anti-microbial prophylaxis" OR "antimicrobial prophylaxis" OR "anti-bacterial prophylaxis" OR "antibacterial prophylaxis" (7)

- "surgical wound infection" OR "surgical wound infections" OR "surgical infection" OR "surgical infections" OR "surgical contamination" OR "surgical contaminations" (4)

- "postoperative wound infection" OR "postoperative wound infection" OR "postoperative wound infections" OR "post-operative wound infections" (0)

- "postoperative infection" OR "post-operative infection" OR "postoperative infections" OR "post-operative infections" (0).

\section{Internet}

The Internet searching was carried out on 1 December 2005 through the specialist search engine OMNI and the meta-search engine Copernic.

\section{OMNI: Internet (http://www.omni.ac.uk)}

The web resources identified through OMNI were scanned and eight potentially relevant web pages were downloaded for consideration by the reviewers.

The search interface for OMNI allows only simple searching. The following terms were entered lineby-line:
- vancomycin OR vancocin OR lyphocin OR vancoled OR vancor OR teicoplanin OR teichomycin OR teichomycins OR targocid OR glycopeptide OR glycopeptides (1)

- "antibiotic prophylaxis" (8)

- "anti-microbial prophylaxis" (0)

- "antimicrobial prophylaxis" (2)

- "anti-bacterial prophylaxis" (0)

- "antibacterial prophylaxis" (0)

- "surgical wound infection" (3)

- "surgical wound infections" (0)

- "surgical infection" (0)

- "surgical infections" (0)

- "surgical contamination" (0)

- "surgical contaminations" (0)

- "postoperative wound infection" (0)

- "post-operative wound infection" (0)

- "postoperative wound infections" (0)

- "post-operative wound infections" (0)

- "postoperative infection" (0)

- "post-operative infection" (0)

- "postoperative infections" (0)

- "post-operative infections" (0).

\section{Copernic: Internet (http://www.copernic.com)}

The web resources identified through Copernic were scanned and 15 potentially relevant web pages were downloaded for consideration by the reviewers.

The search interface for Copernic allows only simple searching. The following terms were entered line-by-line:

- vancomycin vancocin lyphocin vancoled vancor teicoplanin teichomycin teichomycins targocid glycopeptide glycopeptides [Any word] (41)

- "antibiotic prophylaxis" surgery [All words] (36)

- "anti-microbial prophylaxis" surgery [All words] (33)

- "antimicrobial prophylaxis" surgery [All words] (38)

- "anti-bacterial prophylaxis" surgery [All words] (18)

- "antibacterial prophylaxis" surgery [All words] (45)

- surgical wound infection [Exact phrase] (37)

- surgical wound infections [Exact phrase] (42)

- surgical infection [Exact phrase] (37)

- surgical infections [Exact phrase] (40)

- surgical contamination [Exact phrase] (41)

- postoperative wound infection [Exact phrase] (46)

- post-operative wound infection [Exact phrase] (43)

- postoperative wound infections [Exact phrase] (41) 
- post-operative wound infections [Exact phrase] (38)

- postoperative infection [Exact phrase] (41)

- post-operative infection [Exact phrase] (47)

- postoperative infections [Exact phrase] (38)

- post-operative infections [Exact phrase] (38).

\section{Economic evaluations}

Economic evaluations were identified through the strategies run in the resources listed above, along with further searches of NHS EED, HEED and IDEAS. The strategies used to identify economic evaluations in these databases are listed below.

\section{NHS EED: Internal CRD Database}

The NHS EED search was carried out on 18 October 2005, using the CRD's internal search interface. The search identified 140 records.

Date limits were not set within the search as pre1990 records were deleted within the endnote library.

1. S vancomycin or vancocin or lyphocin or vancoled or vancor or teicoplanin or teichomycin $\$$ or targocid or glycopeptide $\$(88)$

2. $S$ antibiotic $\$(2 \mathrm{w})$ (prophyla $\$$ or premedicat $\$$ or pre-medicat\$ or therapeutic $\$$ ) (88)

3. S ((anti-microbial\$ or antimicrobial $\$)(2 \mathrm{w})($ prophyla $\$$ or premedicat $\$$ or pre-medicat $\$$ or therapeutic $\$$ )) (8)

4. S ((anti-bacterial\$ or antibacterial $\$)(2 \mathrm{w})$ (prophyla $\$$ or premedicat $\$$ or pre-medicat $\$$ or therapeutic $\$)$ ) (2)

5. S ((anti-mycobacterial\$ or antimycobacterial $\$)(2 \mathrm{w})$ (prophyla $\$$ or premedicat $\$$ or pre-medicat $\$$ or therapeutic $\$)$ ) (0)

6. S ((bacteriocidal or bacteriocide $\$)(2 \mathrm{w})$ (prophyla $\$$ or premedicat $\$$ or pre-medicat $\$$ or therapeutic $\$$ ) ) (0)

7. s S1 or s2 or s3 or s4 or s5 or s6 (140)

\section{HEED: CD-ROM (issue October 2005)}

The HEED search was carried out on 19 October 2005 and identified 244 records.

Date limits were not set within the search as pre1990 records were deleted within the endnote library.

1. $\mathrm{AX}=$ vancomycin $\mathrm{OR}$ vancocin $\mathrm{OR}$ lyphocin OR vancoled $O R$ vancOR OR teicoplanin $O R$ teichomycin* OR targocid OR glycopeptide* (144)
2. $\mathrm{AX}=$ 'antibiotic prophylaxis' within $2 \mathrm{OR}$ 'antibiotic premedication' within $2 \mathrm{OR}$ 'antibiotic pre-medication' within $2 \mathrm{OR}$ 'antibiotic therapeutic' within 2 (78)

3. $\mathrm{AX}=$ 'antibiotics prophylaxis' within $2 \mathrm{OR}$ 'antibiotics premedication' within 2 OR 'antibiotics pre-medication' within 2 OR 'antibiotics therapeutic' within 2 (5)

4. $\mathrm{AX}=$ 'anti-microbial prophylaxis' within $2 \mathrm{OR}$ 'anti-microbial premedication' within $2 \mathrm{OR}$ 'anti-microbial pre-medication' within $2 \mathrm{OR}$ 'anti-microbial therapeutic' within $2(0)$

5. $\mathrm{AX}=$ 'anti-microbials prophylaxis' within $2 \mathrm{OR}$ 'anti-microbials premedication' within 2 OR 'anti-microbials pre-medication' within $2 \mathrm{OR}$ 'anti-microbials therapeutic' within $2(0)$

6. $\mathrm{AX}=$ 'antimicrobial prophylaxis' within $2 \mathrm{OR}$ 'antimicrobial premedication' within $2 \mathrm{OR}$ 'antimicrobial pre-medication' within $2 \mathrm{OR}$ 'antimicrobial therapeutic' within 2 (27)

7. $\mathrm{AX}=$ 'antimicrobials prophylaxis' within $2 \mathrm{OR}$ 'antimicrobials premedication' within $2 \mathrm{OR}$ 'antimicrobials pre-medication' within 2 OR 'antimicrobials therapeutic' within 2 (1)

8. $\mathrm{AX}=$ 'anti-bacterial prophylaxis' within $2 \mathrm{OR}$ 'anti-bacterial premedication' within $2 \mathrm{OR}$ ‘anti-bacterial pre-medication' within 2 OR 'anti-bacterial therapeutic' within 2 (1)

9. $\mathrm{AX}=$ 'anti-bacterials prophylaxis' within $2 \mathrm{OR}$ ‘anti-bacterials premedication' within 2 OR 'anti-bacterials pre-medication' within 2 OR 'anti-bacterials therapeutic' within 2 (0)

10. $\mathrm{AX}=$ 'antibacterial prophylaxis' within $2 \mathrm{OR}$ 'antibacterial premedication' within $2 \mathrm{OR}$ 'antibacterial pre-medication' within 2 OR 'antibacterial therapeutic' within 2 (2)

11. $\mathrm{AX}=$ 'antibacterials prophylaxis' within $2 \mathrm{OR}$ 'antibacterials premedication' within 2 OR 'antibacterials pre-medication' within 2 OR 'antibacterials therapeutic' within $2(0)$

12. $\mathrm{AX}=$ 'anti-mycobacterial prophylaxis' within 2 OR 'anti-mycobacterial premedication' within 2 OR 'anti-mycobacterial pre-medication' within 2 OR 'anti-mycobacterial therapeutic' within $2(0)$

13. $\mathrm{AX}=$ 'anti-mycobacterials prophylaxis' within 2 OR 'anti-mycobacterials premedication' within 2 OR 'anti-mycobacterials pre-medication' within 2 OR 'anti-mycobacterials therapeutic' within $2(0)$

14. $\mathrm{AX}=$ 'antimycobacterial prophylaxis' within 2 OR 'antimycobacterial premedication' within 2 OR 'antimycobacterial pre-medication' within 2 OR 'antimycobacterial therapeutic' within 2 (0)

15. $\mathrm{AX}=$ 'antimycobacterials prophylaxis' within 2 OR 'antimycobacterials premedication' within 
2 OR 'antimycobacterials pre-medication' within 2 OR 'antimycobacterials therapeutic' within $2(0)$

16. $\mathrm{AX}=$ 'bacteriocidal prophylaxis' within $2 \mathrm{OR}$ 'bacteriocidal premedication' within 2 OR 'bacteriocidal pre-medication' within 2 OR 'bacteriocidal therapeutic' within $2(0)$

17. $\mathrm{AX}=$ 'bacteriocide prophylaxis' within $2 \mathrm{OR}$ 'bacteriocide premedication' within 2 OR 'bacteriocide pre-medication' within 2 OR 'bacteriocide therapeutic' within $2(0)$

18. $\mathrm{AX}=$ 'bacteriocides prophylaxis' within $2 \mathrm{OR}$ 'bacteriocides premedication' within 2 OR 'bacteriocides pre-medication' within 2 OR 'bacteriocides therapeutic' within $2(0)$

19. $\mathrm{CS}=1$ or 2 or 3 or 4 or 5 or 6 or 7 or 8 or 9 or 10 or 11 or 12 or 13 or 14 or 15 or 16 or 17 or 18 (244)

\section{IDEAS: Internet (http://ideas.repec.org)}

The IDEAS search was carried out on 20 October 2005. No relevant papers were identified.

The search interface for IDEAS allows only simple searching. The following terms were entered lineby-line:

- vancomycin or vancocin or lyphocin or vancoled or vancor or teicoplanin or teichomycin* or targocid or glycopeptide* $(0)$

- antibiotic* and (prophyla* or premedicat* or pre-medicat* or therapeutic*) (0)

- anti-microbial* and (prophyla* or premedicat* or pre-medicat* or therapeutic*) $(0)$

- antimicrobial* and (prophyla* or premedicat* or pre-medicat* or therapeutic*) (0)

- anti-bacterial* and (prophyla* or premedicat* or pre-medicat* or therapeutic*) $(0)$

- antibacterial* and (prophyla* or premedicat* or pre-medicat* or therapeutic*) $(0)$

- anti-mycobacterial* and (prophyla* or premedicat* or pre-medicat* or therapeutic*) $(0)$

- antimycobacterial* and (prophyla* or premedicat* or pre-medicat* or therapeutic*) (0)

- bacteriocidal and (prophyla* or premedicat* or pre-medicat* or therapeutic*) (0)

- bacteriocide* and (prophyla* or premedicat* or pre-medicat* or therapeutic*) $(0)$

\section{Economic model}

Restricted searches to inform the economic model were undertaken.

Searches were performed on NHS EED via the CRD's internal search interface, to identify:

- economic evaluations of glycopeptides versus non-glycopeptides

- economic evaluations on prophylaxis for surgery

- economic evaluations that assess the prevalence of surgical site infections

- economic evaluations of antimicrobial resistance.

Searches were performed on MEDLINE via Ovid (http://gateway.ovid.com/athens), to identify:

- papers on epidemiological modelling

- papers on decision analysis and antibiotics.

Searches were performed on MEDLINE and EMBASE via Ovid

(http://gateway.ovid.com/athens), to identify:

- papers on treatments used when antimicrobial resistance is a problem

- conceptual papers on evaluating the impact of MRSA/antimicrobial resistance.

Searches were performed on MEDLINE, EMBASE and CINAHL via Ovid

(http://gateway.ovid.com/athens) and NHS EED via the CRD's internal search interface, to identify:

- papers on modelling resistance.

Full details of the strategies used to inform the economic model are available from the CRD (tel. 01904 321846; email crd-info@york.ac.uk). 


\section{Appendix 2}

\section{Quality assessment checklists}

\section{Effectiveness review}

1. Was the assignment to treatment groups really random?

Adequate approaches to sequence generation:

- Computer-generated random numbers

- Random numbers tables.

Inadequate approaches to sequence generation:

- Use of alternation, case record numbers, birth dates or week days.

2. Method of randomisation.

3. Was the allocation of treatment concealed?

Adequate approaches to concealment of randomisation:

- Centralised or pharmacy-controlled randomisation

- Serially numbered identical containers

- On-site computer based system with a randomisation sequence that is not readable until allocation

- Other approaches with robust methods to prevent foreknowledge of the allocation sequence to clinicians and patients.

Inadequate approaches to concealment of randomisation:

- Use of alternation, case record numbers, birth dates or week days

- Open random numbers lists

- Serially numbered envelopes (even sealed opaque envelopes can be subject to manipulation).

4. Were the groups similar at baseline?

5. Were eligibility criteria specified?

6. Were outcome assessors blinded to treatment?

7. Was the patient blinded to treatment?

8. Were analyses on an intention-to-treat basis?

9. Was there an appropriate sample size calculation?

10. Were withdrawals reported?

\section{Cost-effectiveness review}

Studies of cost-effectiveness will be assessed using the following criteria, which is an updated version of the checklist developed by Drummond and colleagues: ${ }^{34}$

\section{Study question}

1. Costs and effects examined.
2. Alternatives compared.

3. The viewpoint(s)/perspective of the analysis is clearly stated (e.g. NHS, society).

\section{Selection of alternatives}

4. All relevant alternatives are compared (including do nothing if applicable).

5 . The alternatives being compared are clearly described (who did what, to whom, where and how often).

6. The rationale for choosing the alternative programmes or interventions compared is stated.

\section{Form of evaluation}

7. The choice of form of economic evaluation is justified in relation to the questions addressed.

8. If a cost-minimisation design is chosen, have equivalent outcomes been adequately demonstrated?

\section{Effectiveness data}

9. The source(s) of effectiveness estimates used are stated (e.g. single study, selection of studies, systematic review, expert opinion).

10. Effectiveness data from RCT or review of RCTs.

11. Potential biases identified (especially if data not from RCTs).

12. Details of the method of synthesis or metaanalysis of estimates are given (if based on an overview of a number of effectiveness studies).

\section{Costs}

13. All the important and relevant resource use included.

14. All the important and relevant resource use measured accurately (with methodology).

15. Appropriate unit costs estimated (with methodology).

16. Unit costs reported separately from resource use data.

17. Productivity costs treated separately from other costs.

18. The year and country to which unit costs apply is stated with appropriate adjustments for inflation and/or currency conversion. 


\section{Benefit measurement and valuation}

19. The primary outcome measure(s) for the economic evaluation are clearly stated (cases detected, life-years, QALYs, etc.).

20. Methods to value health states and other benefits are stated (e.g. time trade-off).

21. Details of the individuals from whom valuations were obtained are given (patients, members of the public, healthcare professionals, etc.).

\section{Decision modelling}

22. Details of any decision model used are given (e.g. decision tree, Markov model).

23. The choice of model used and the key input parameters on which it is based are adequately detailed and justified.

24. All model outputs described adequately.

\section{Discounting}

25. Discount rate used for both costs and benefits.

26. Do discount rates accord with NHS guidance (1.5-2\% for benefits; $6 \%$ for costs)?

\section{Allowance for uncertainty Stochastic analysis of patient-level data}

27. Details of statistical tests and CIs are given for stochastic data.

28. Uncertainty around cost-effectiveness expressed (e.g. CI around ICER, cost-effectiveness acceptability curves).

29. Sensitivity analysis used to assess uncertainty in non-stochastic variables (e.g. unit costs, discount rates) and analytic decisions (e.g. methods to handle missing data).

\section{Stochastic analysis of decision models}

30. Are all appropriate input parameters included with uncertainty?

31. Is second-order uncertainty (uncertainty in means) included rather than first-order uncertainty (uncertainty between patients)?

32. Are the probability distributions adequately detailed and appropriate?

33. Sensitivity analysis used to assess uncertainty in non-stochastic variables (e.g. unit costs, discount rates) and analytic decisions (e.g. methods to handle missing data).

\section{Deterministic analysis}

34. The approach to sensitivity analysis is given (e.g. univariate, threshold analysis).

35. The choice of variables for sensitivity analysis is justified.

36. The ranges over which the variables are varied are stated.

\section{Presentation of results}

37. Incremental analysis is reported using appropriate decision rules.

38. Major outcomes are presented in a disaggregated as well as an aggregated form.

39. Applicable to the NHS setting.

All items will be graded as either $\checkmark=$ yes (item adequately addressed), $X=$ no (item not adequately addressed), ? = unclear or not enough information, $\mathrm{NA}=$ not applicable or $\mathrm{NS}=$ not stated. 


\section{Appendix 3}

\section{Studies excluded from the effectiveness review}

\begin{tabular}{|c|c|}
\hline Study & Reason for exclusion \\
\hline Antrum, $1990^{1 / 3}$ & Interim analysis of Kester, $1999^{23}$ \\
\hline Antrum, $1992^{1 / 4}$ & Interim analysis of Kester, $1999^{23}$ \\
\hline Barlas, $1993^{115}$ & CCT but not assessing a glycopeptide (full paper was ordered as no abstract was available) \\
\hline Bayston, $1990^{116}$ & Not adult participants only \\
\hline Bell, $1990^{117}$ & Not a CCT (case series: full paper was ordered as no abstract was available) \\
\hline Brooks, $2002^{118}$ & Not a CCT (prospective cohort study) \\
\hline Bucknell, $2000^{119}$ & $\begin{array}{l}\text { CCT but not assessing a glycopeptide (only MRSA high-risk patients received a } \\
\text { glycopeptide) }\end{array}$ \\
\hline Cone, $2004^{120}$ & Duplicate of Mastronardi, $2004^{121}$ \\
\hline Dazzi, $1994^{122}$ & Comparator not an alternative antibiotic regimen \\
\hline de Lalla, $2000^{123}$ & Comparator not an alternative antibiotic regimen \\
\hline de Lalla, 200। 124 & Discussion paper (full paper was ordered as no abstract was available) \\
\hline De Lucas-Villarrubia, $2004^{125}$ & Not a CCT (prospective cohort study) \\
\hline Exner, $1992^{126}$ & Not a CCT (prospective cohort study) \\
\hline Ferro, $1997^{127}$ & Comparator not an alternative antibiotic regimen \\
\hline Feys, $1997^{128}$ & Comparator not an alternative antibiotic regimen \\
\hline Fontanesi, $|99|^{129}$ & Discussion paper (full paper was ordered as no abstract was available) \\
\hline Franchelli, $1993^{130}$ & Comparator not an alternative antibiotic regimen \\
\hline Friberg, $1990^{131}$ & Not a CCT (case reports: full paper was ordered as no abstract was available) \\
\hline Gadallah, $2000^{132}$ & $\begin{array}{l}\text { Not adult participants only. Primary outcome was peritonitis, SSIs were excluded from the } \\
\text { analysis }\end{array}$ \\
\hline Haines, $1993^{133}$ & Letter \\
\hline Isringhaus, $1992^{134}$ & Not a CCT (pharmacokinetic study: all patients received same dose of glycopeptide) \\
\hline Karran, $1992^{135}$ & Comparator not an alternative antibiotic regimen \\
\hline Lazzarini, $2001^{136}$ & Not a CCT (prospective cohort) \\
\hline Lazzarini, $2003^{137}$ & Does not report a primary outcome (primary outcome is blood drug concentration) \\
\hline Mastronardi, $2004^{121}$ & Not a CCT (retrospective cohort) \\
\hline Mendivil Soto, $2001^{138}$ & Does not report a primary outcome \\
\hline Mini, $1999^{139}$ & Discussion paper (full paper was ordered as no abstract was available) \\
\hline Nehrer, $1998^{140}$ & Not a CCT (prospective cohort) \\
\hline Niederhäuser, $1997^{141}$ & Prophylaxis not started before or during surgery (patients were randomised after surgery) \\
\hline Pear, $1998^{142}$ & Not a CCT (prospective cohort) \\
\hline Periti, $1992^{143}$ & Interim analysis of Periti, $1999^{24}$ \\
\hline Rao, $2004^{144}$ & Not a CCT (prospective cohort) \\
\hline Renz, $1999^{145}$ & $\begin{array}{l}\text { Does not report a primary outcome and glycopeptide vs an alternative antibiotic regimen } \\
\text { not main comparison }\end{array}$ \\
\hline Saginur, $1995^{146}$ & Abstract of Saginur, $2000^{18}$ \\
\hline Santini, $1997^{147}$ & Discussion paper (full paper was ordered as no abstract was available) \\
\hline Sassone, $|99|^{148}$ & Not a CCT (prospective cohort) \\
\hline Seppala, $2004^{149}$ & Not a CCT (prospective cohort) \\
\hline Shimada, $1995^{150}$ & Comparator not an alternative antibiotic regimen \\
\hline Skinner, 2001 ${ }^{151}$ & Not a CCT(case report: full paper was ordered as no abstract was available) \\
\hline Sobaci, $2003^{152}$ & Does not report a primary outcome \\
\hline Steer, $1997^{153}$ & Comparator not an alternative antibiotic regimen \\
\hline Tinelli, $1994^{154}$ & Not a CCT (epidemiological study) \\
\hline Tinelli, $1995^{155}$ & Not a CCT (epidemiological study) \\
\hline Trenholme, $\left.199\right|^{156}$ & Abstract only, not enough information reported to extract outcome data \\
\hline Wilson, $1990^{157}$ & Discussion paper: reports results of two trials but both published pre- 1990 \\
\hline Zibari, $1997^{158}$ & Comparator not an alternative antibiotic regimen \\
\hline
\end{tabular}





\section{Appendix 4}

\section{Data extraction tables: effectiveness review}




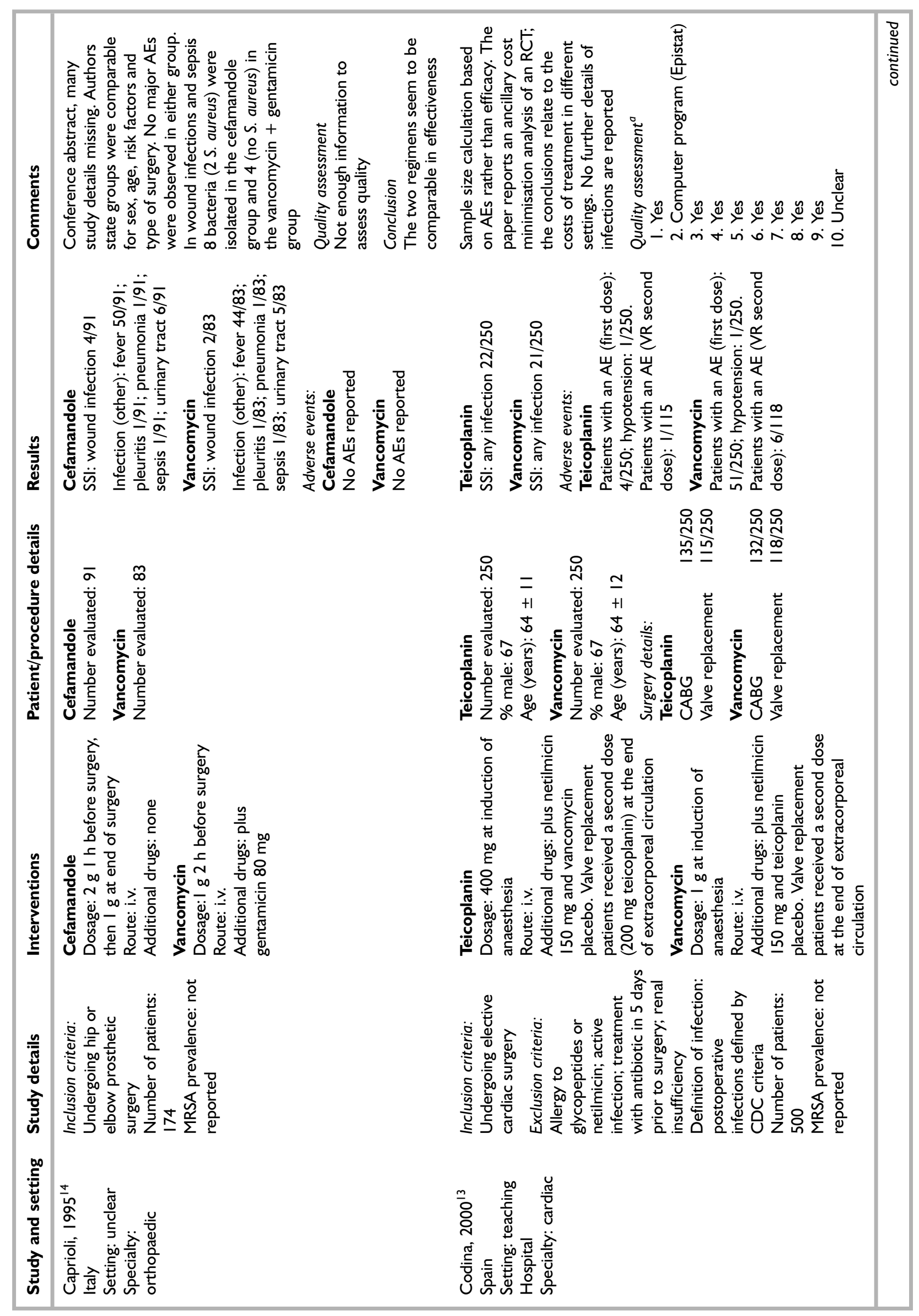




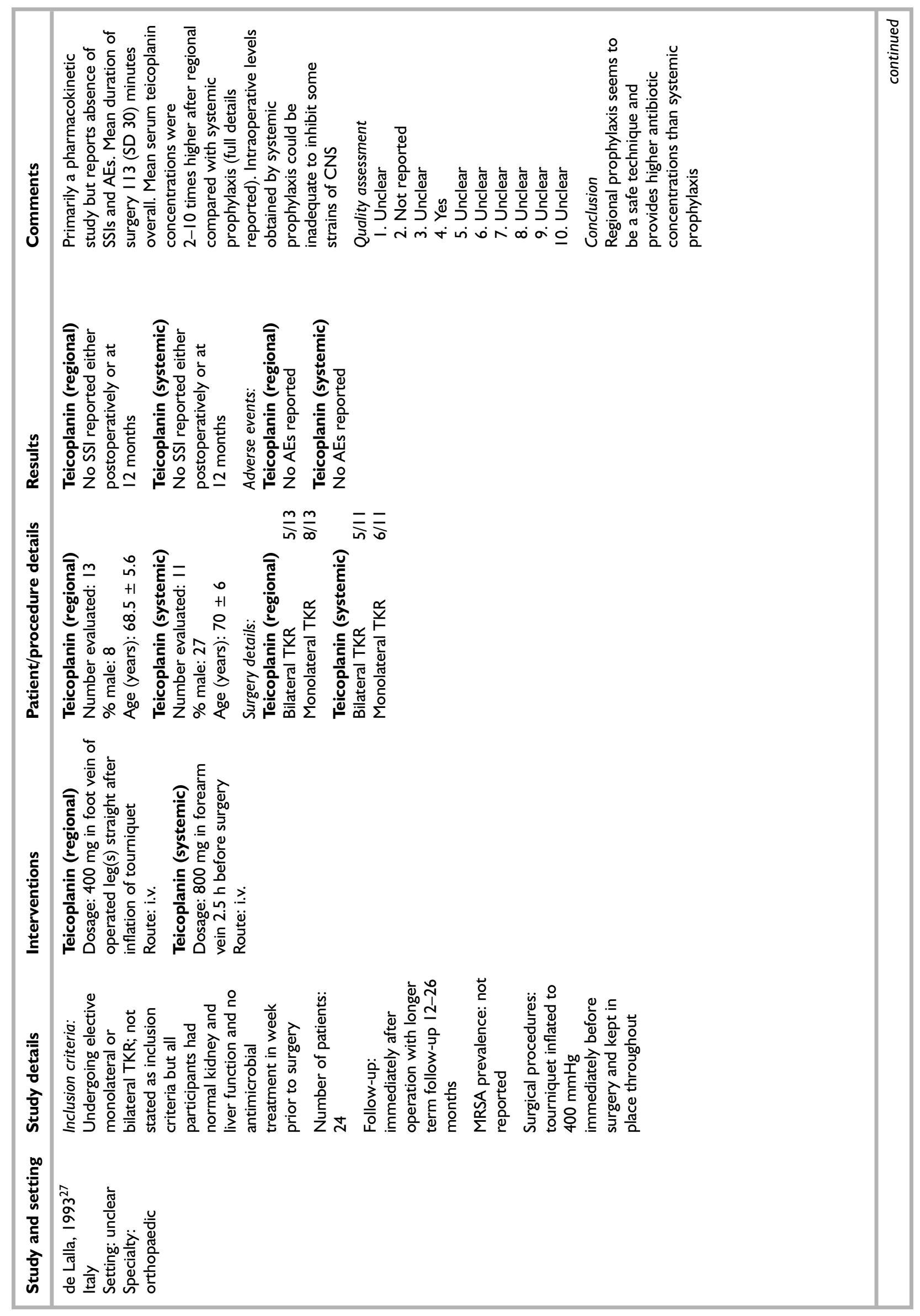




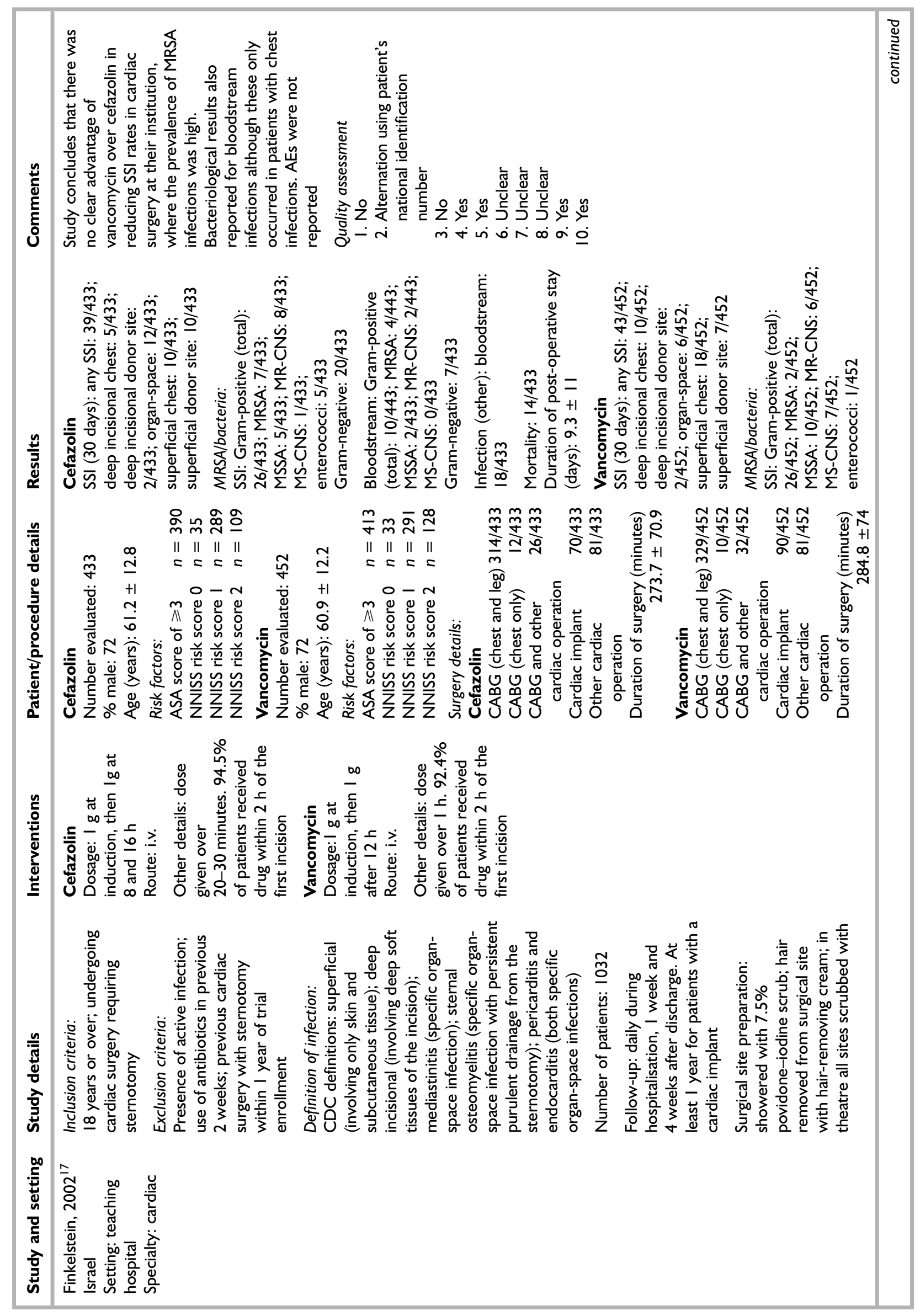




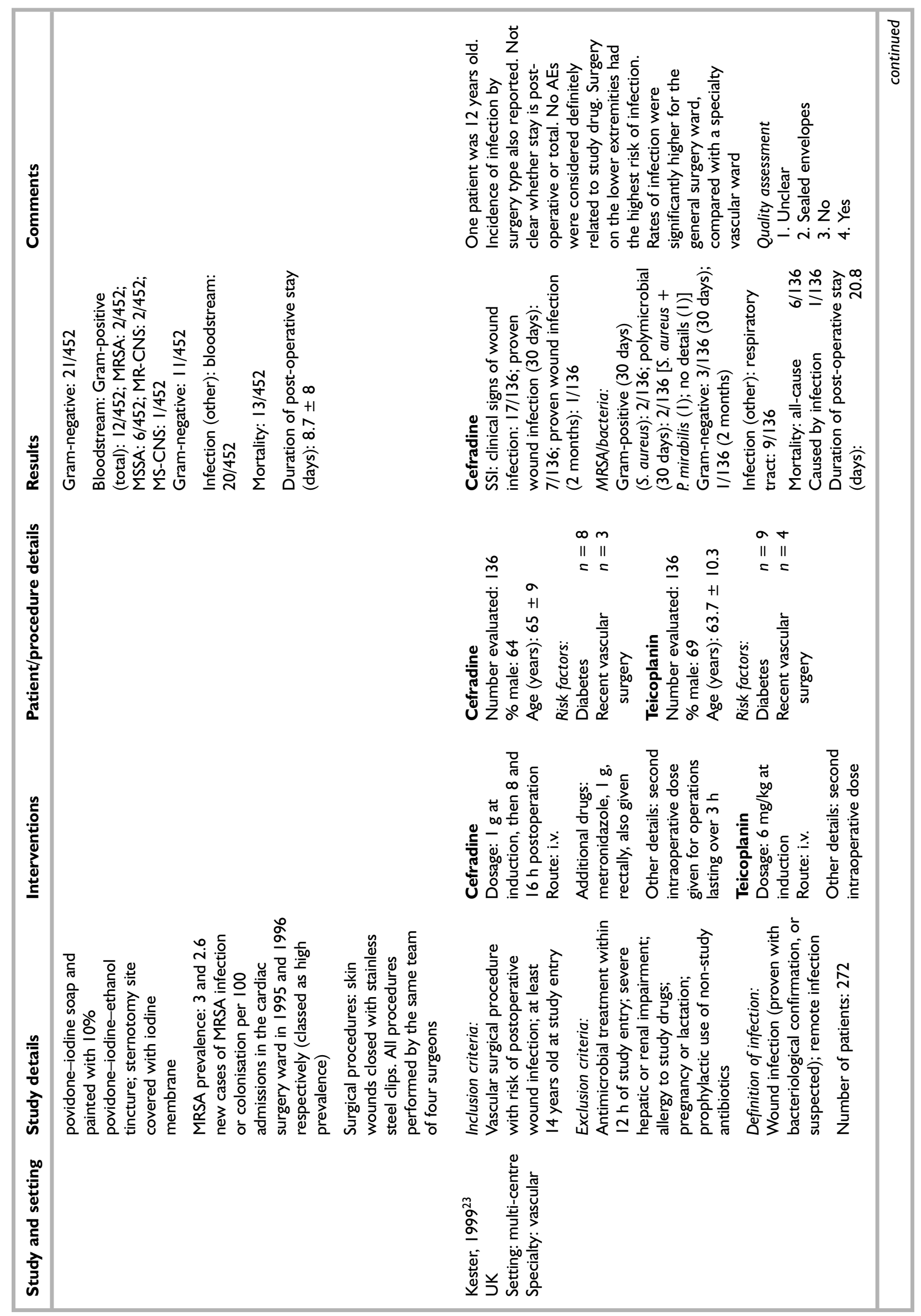




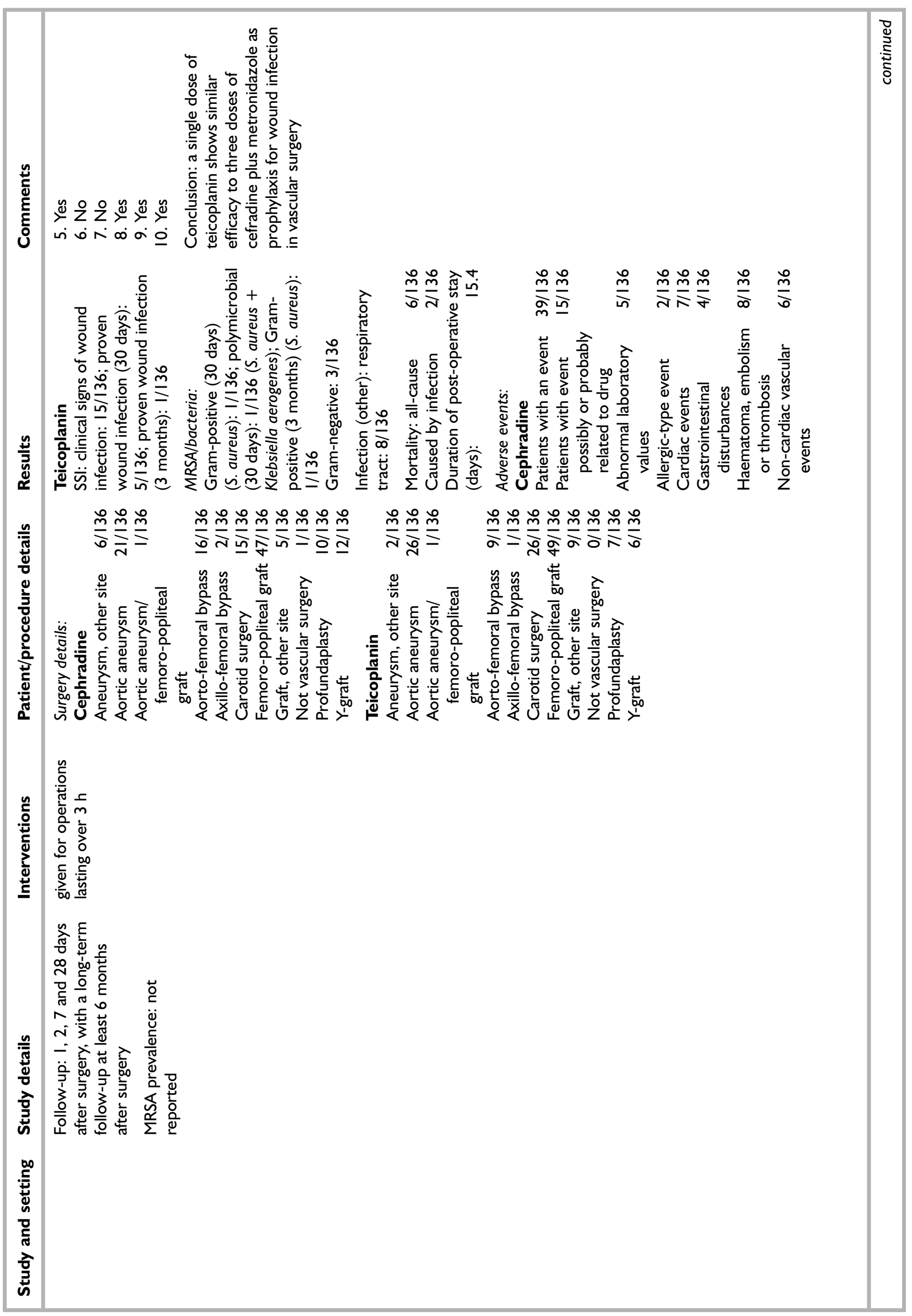




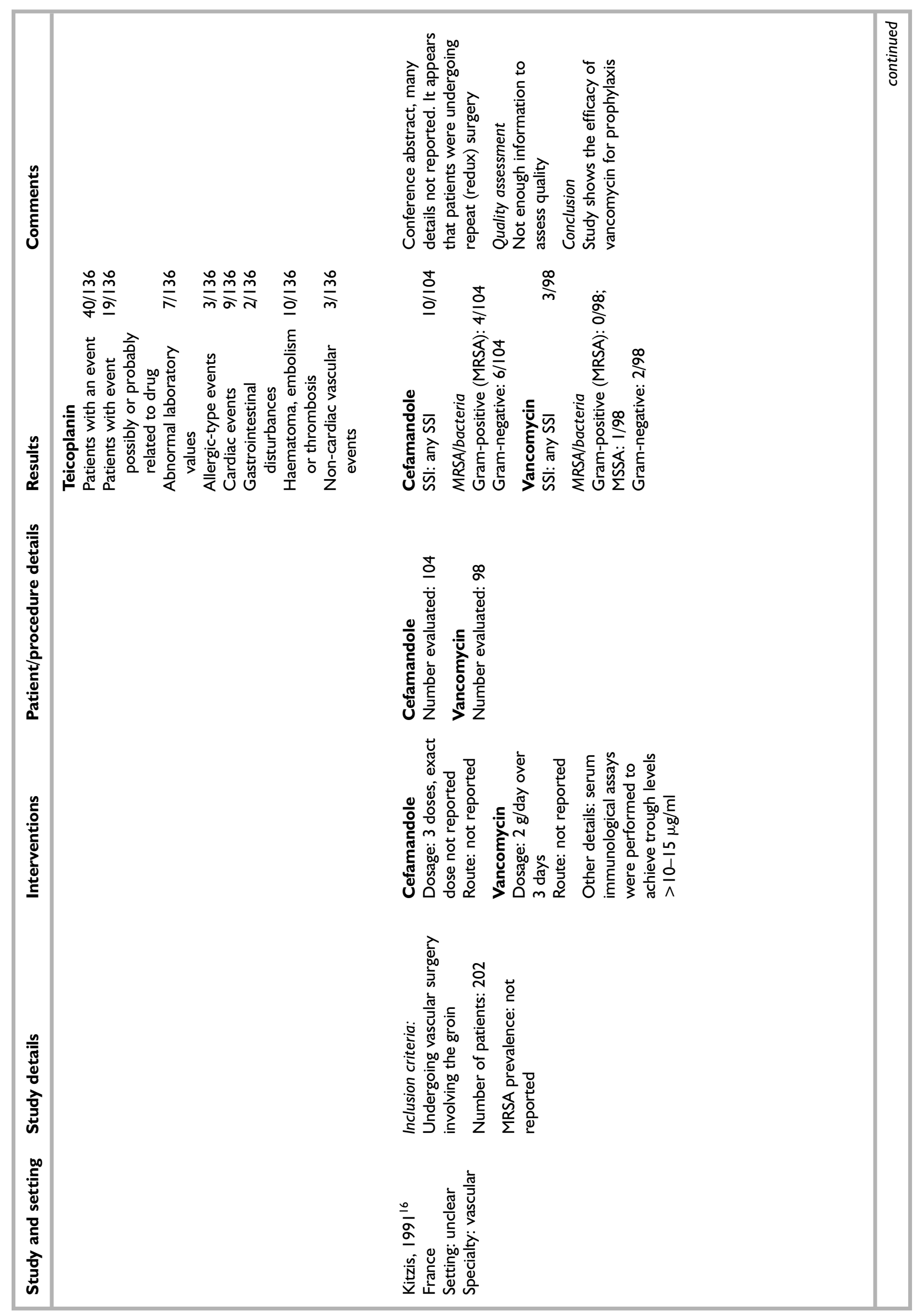




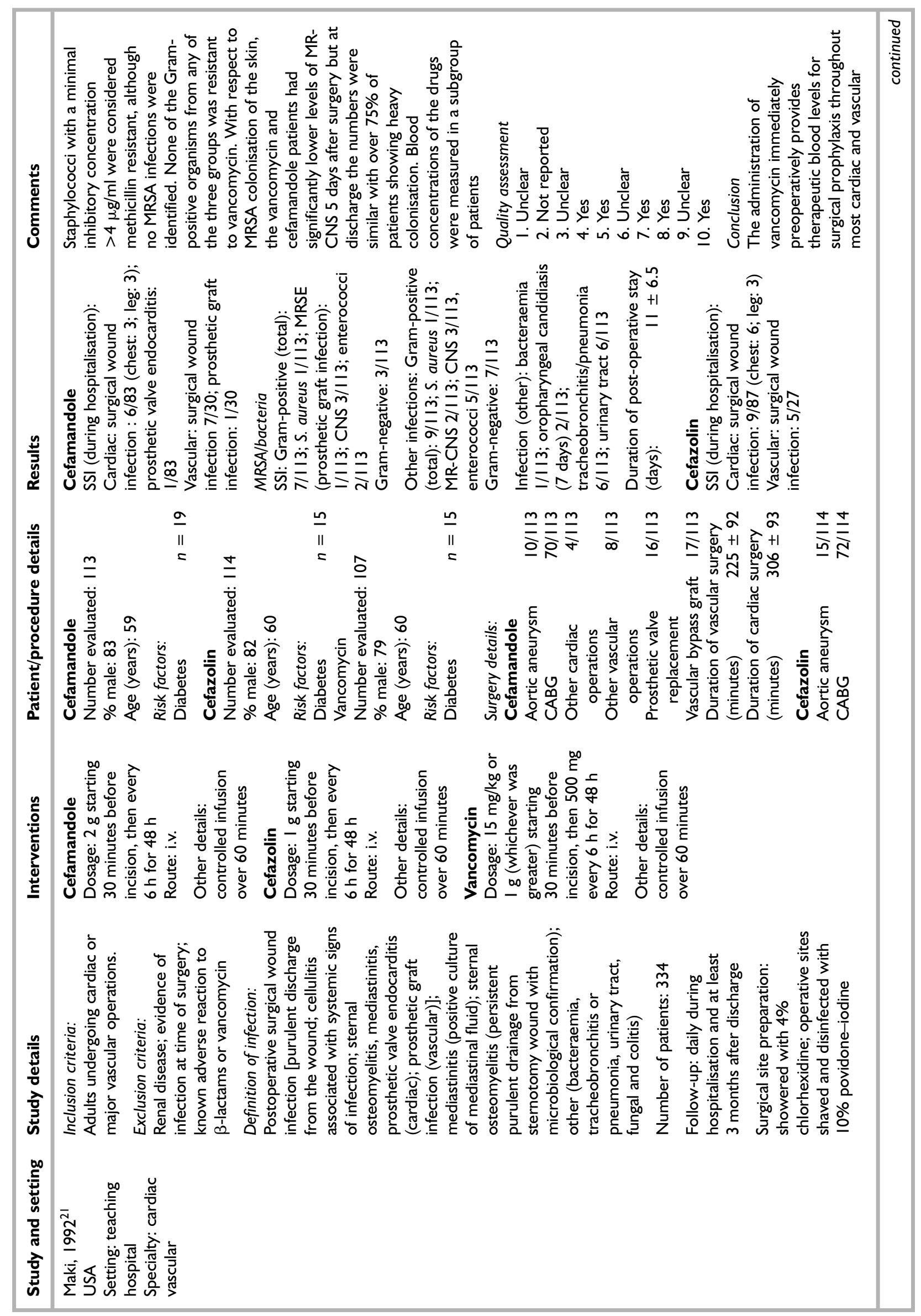




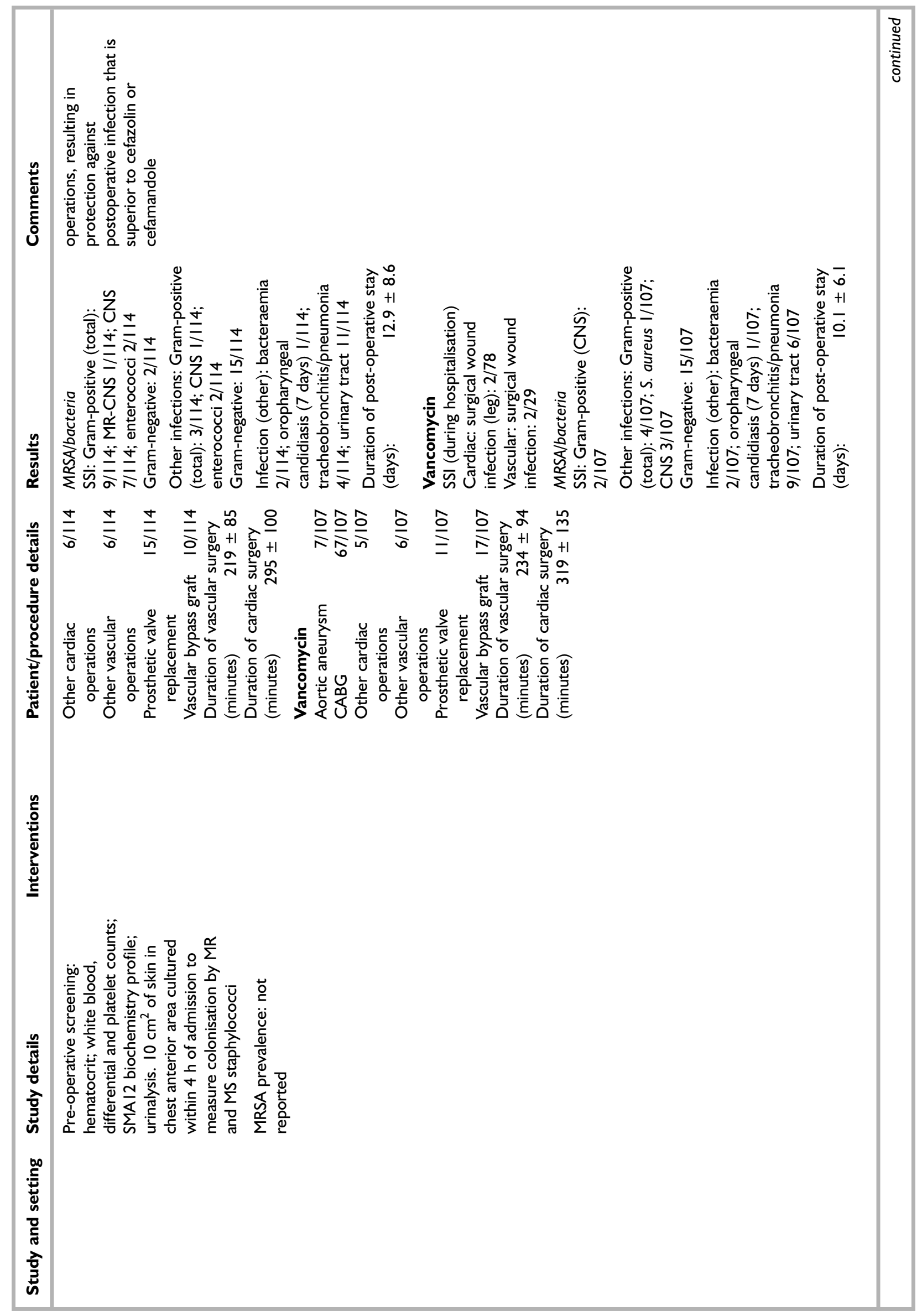




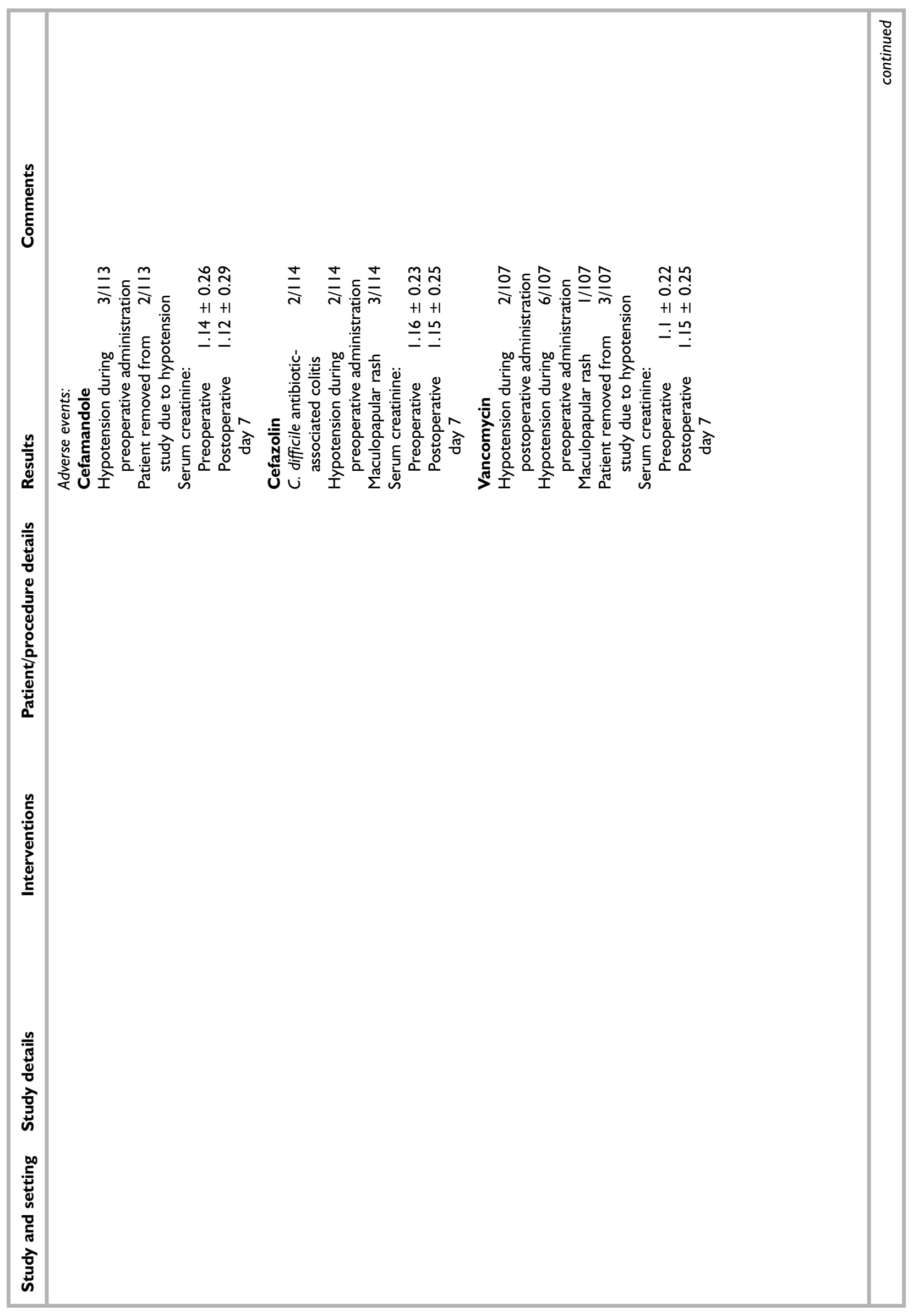




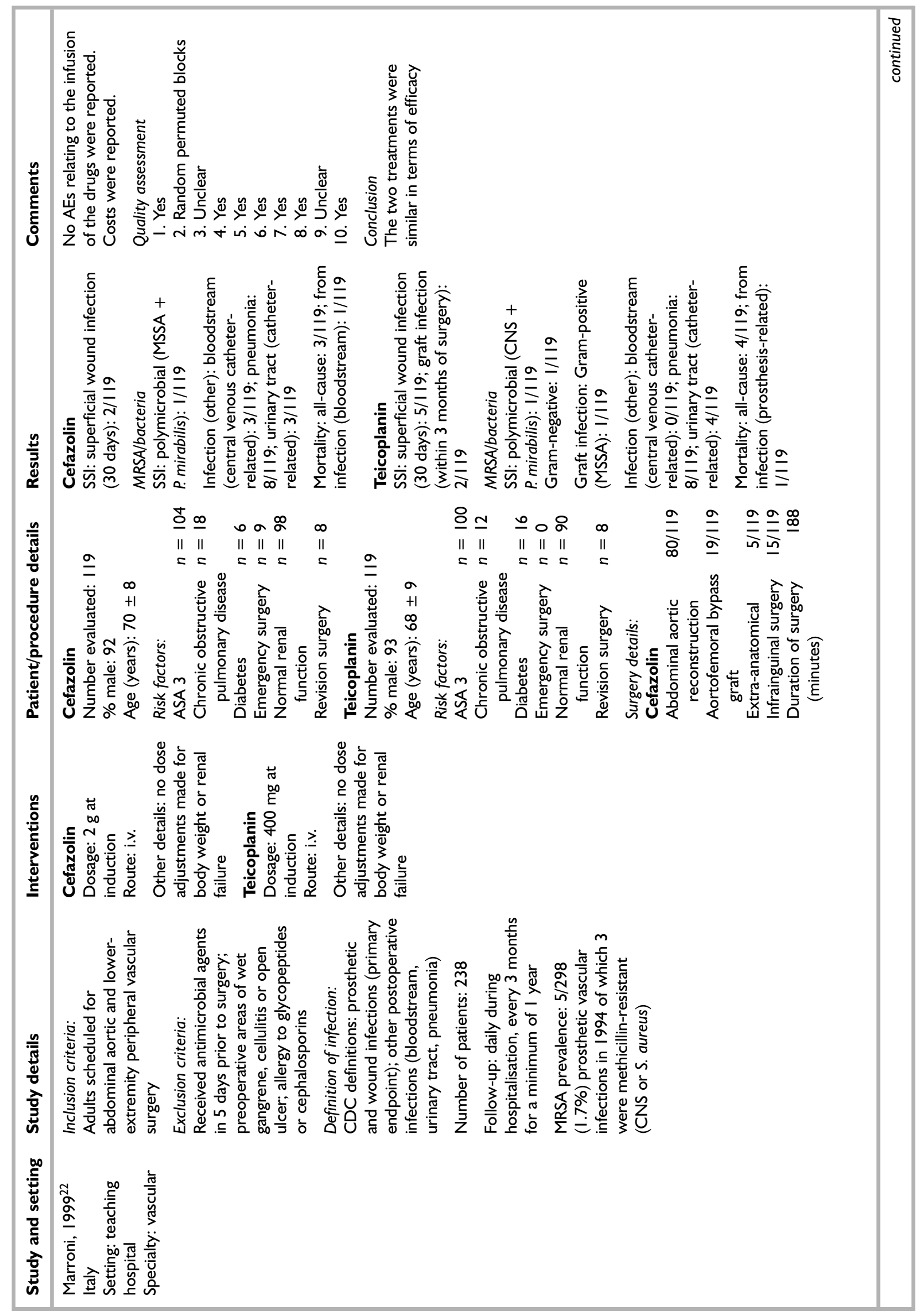




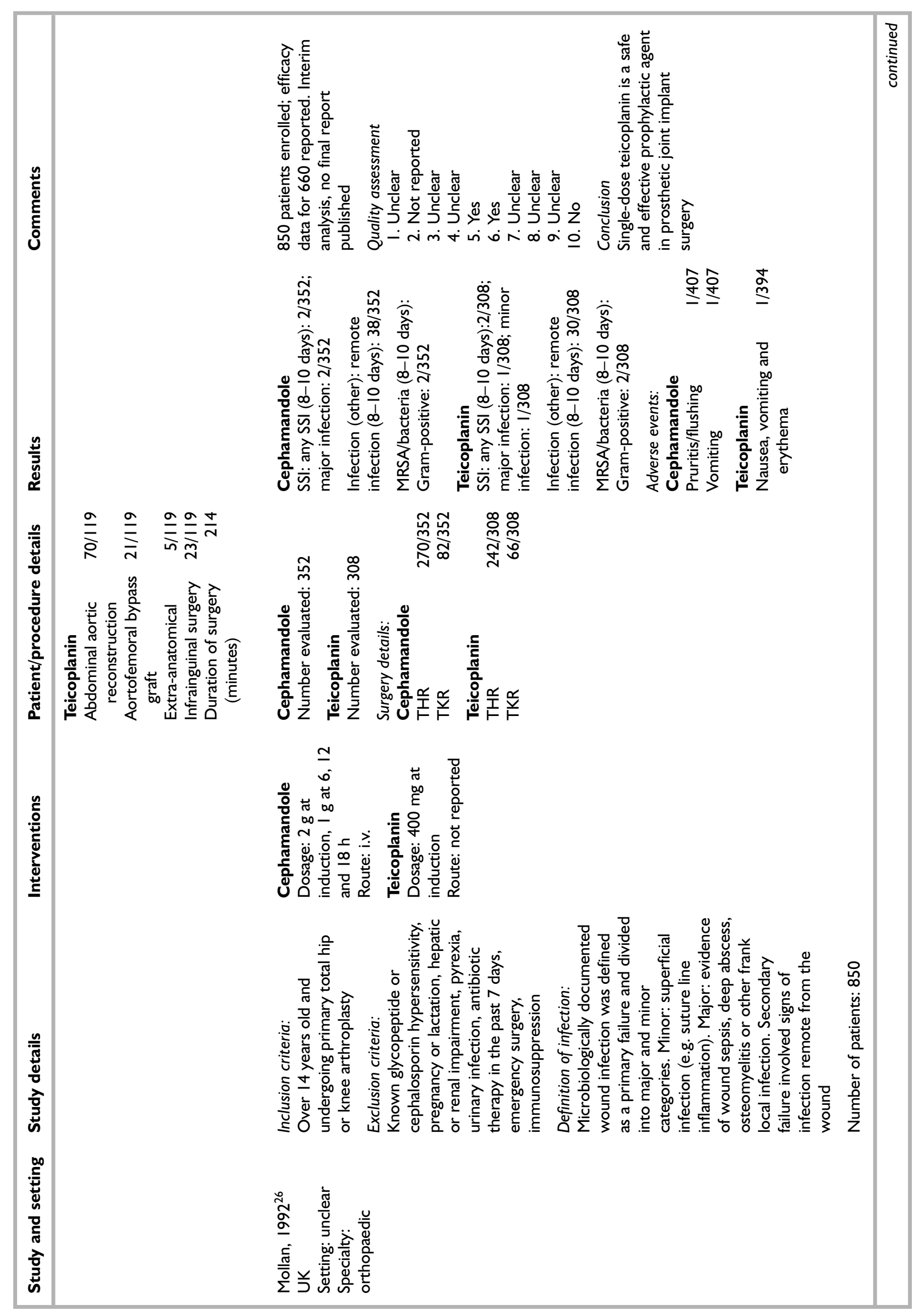




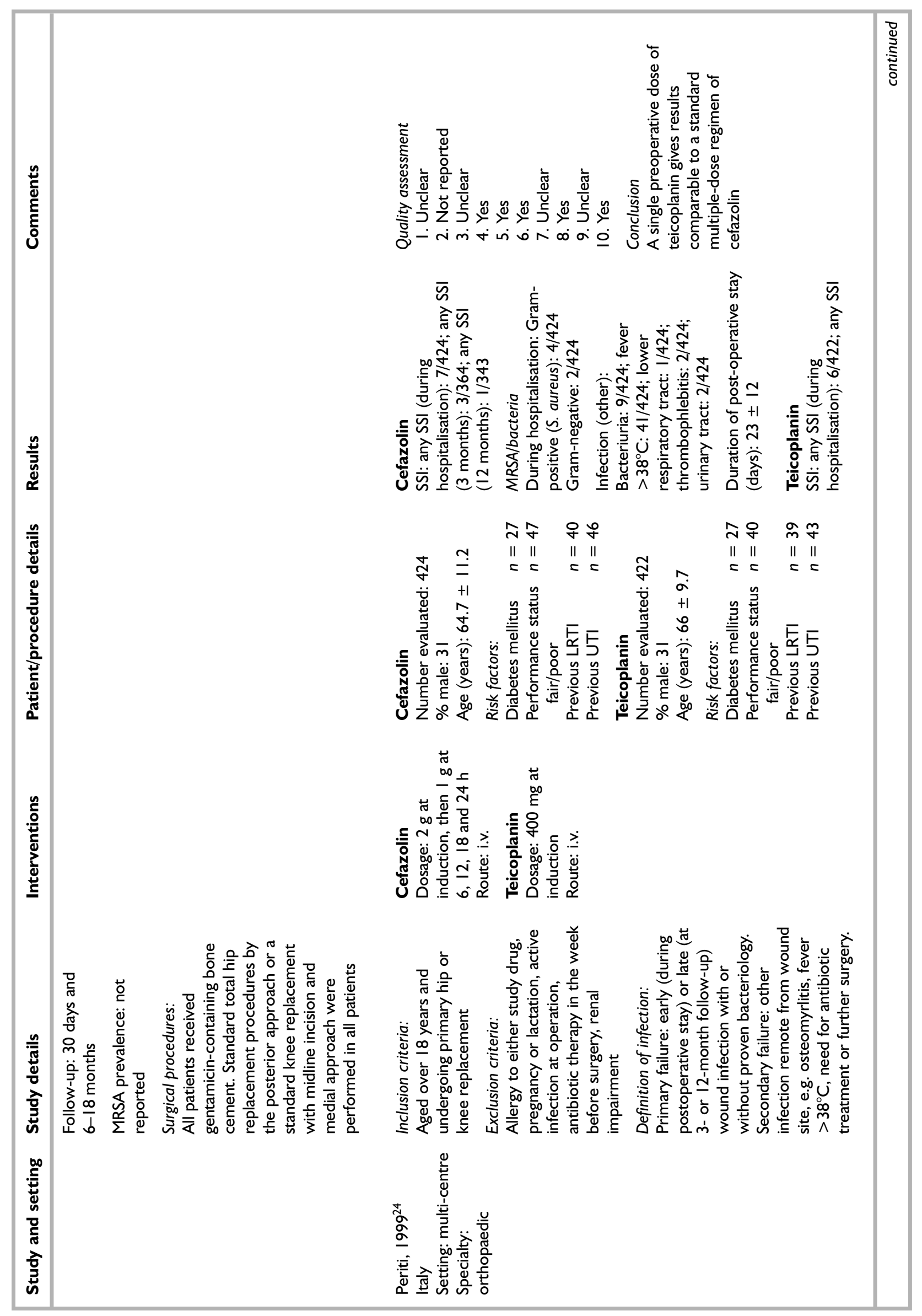




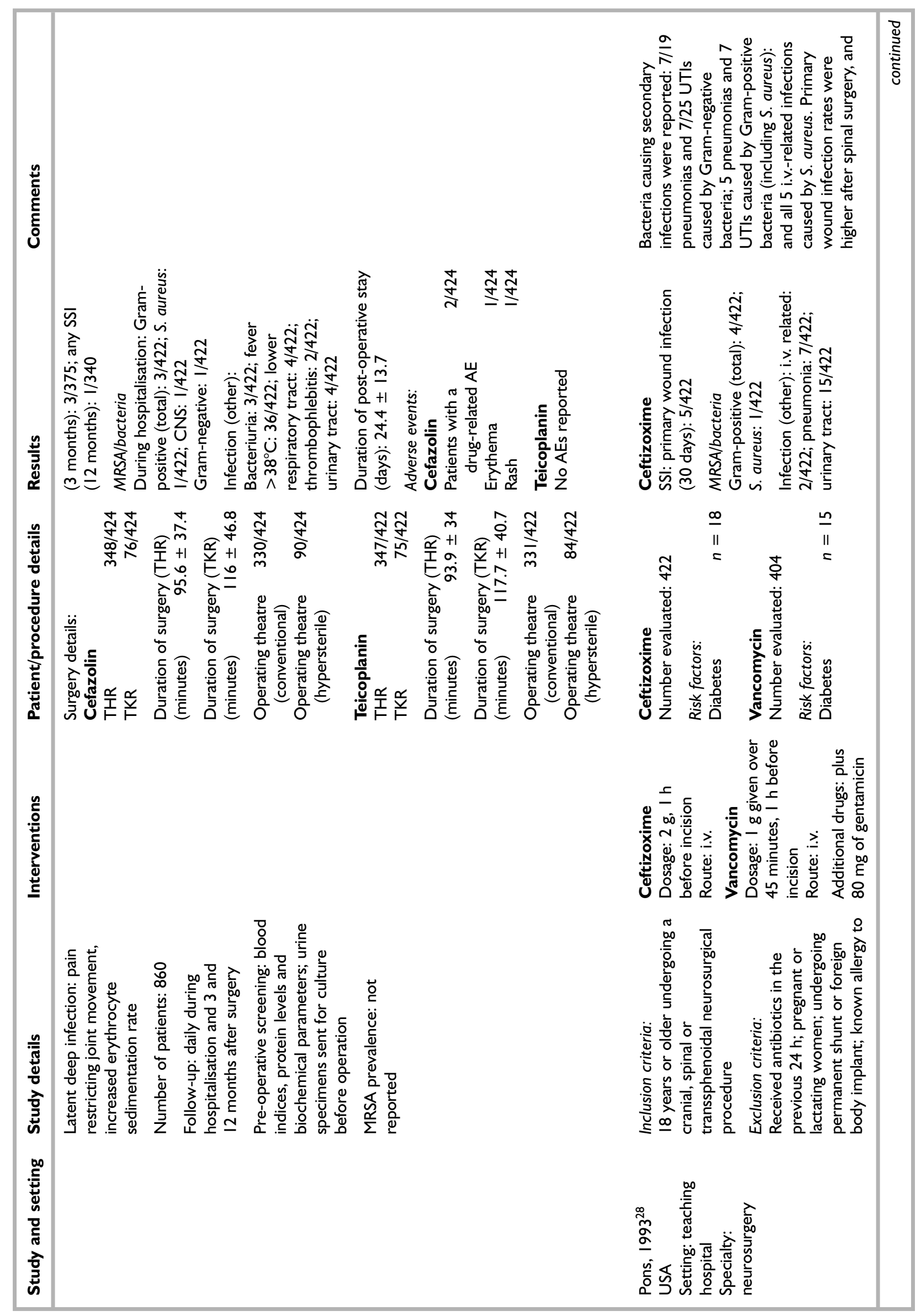




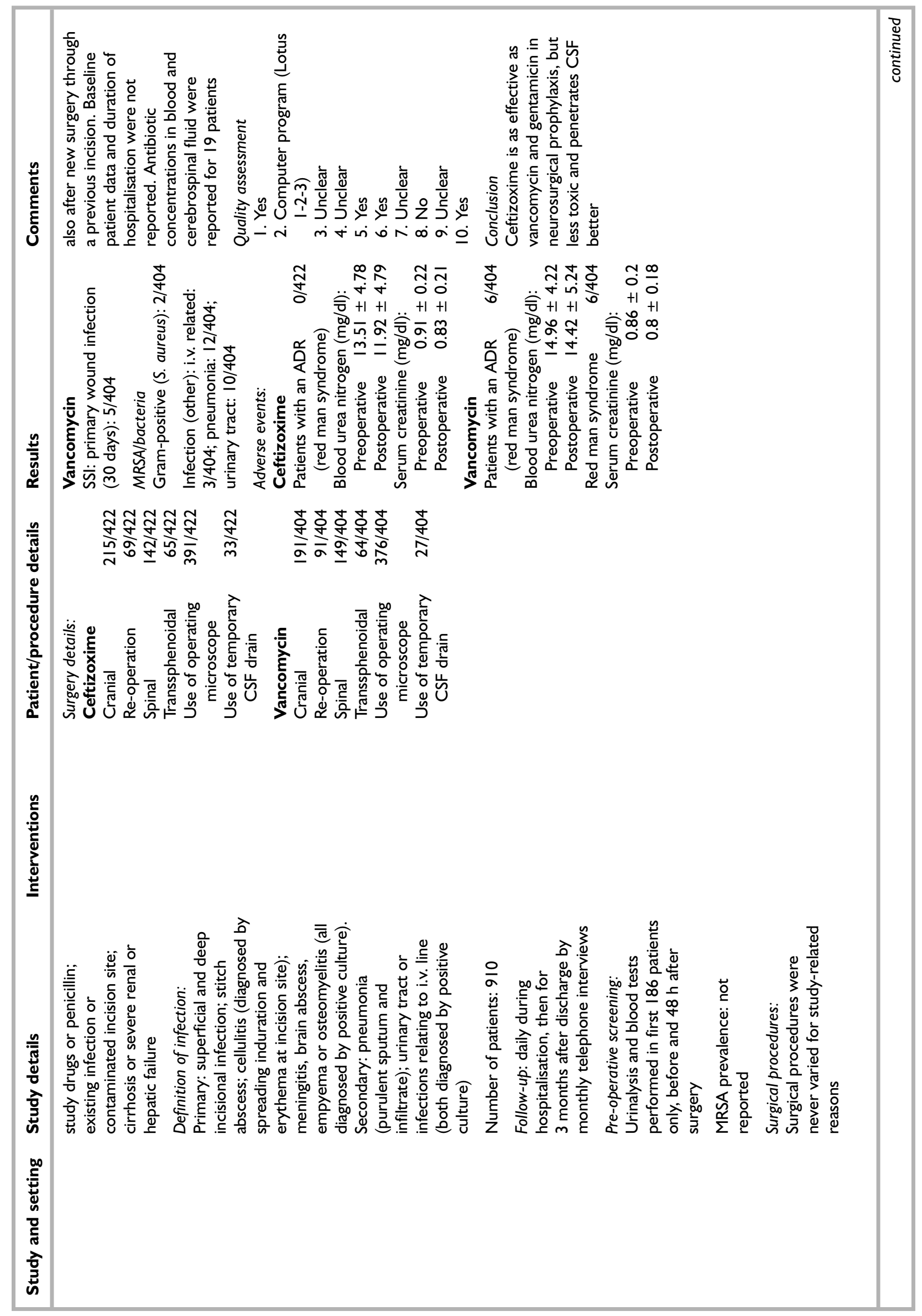




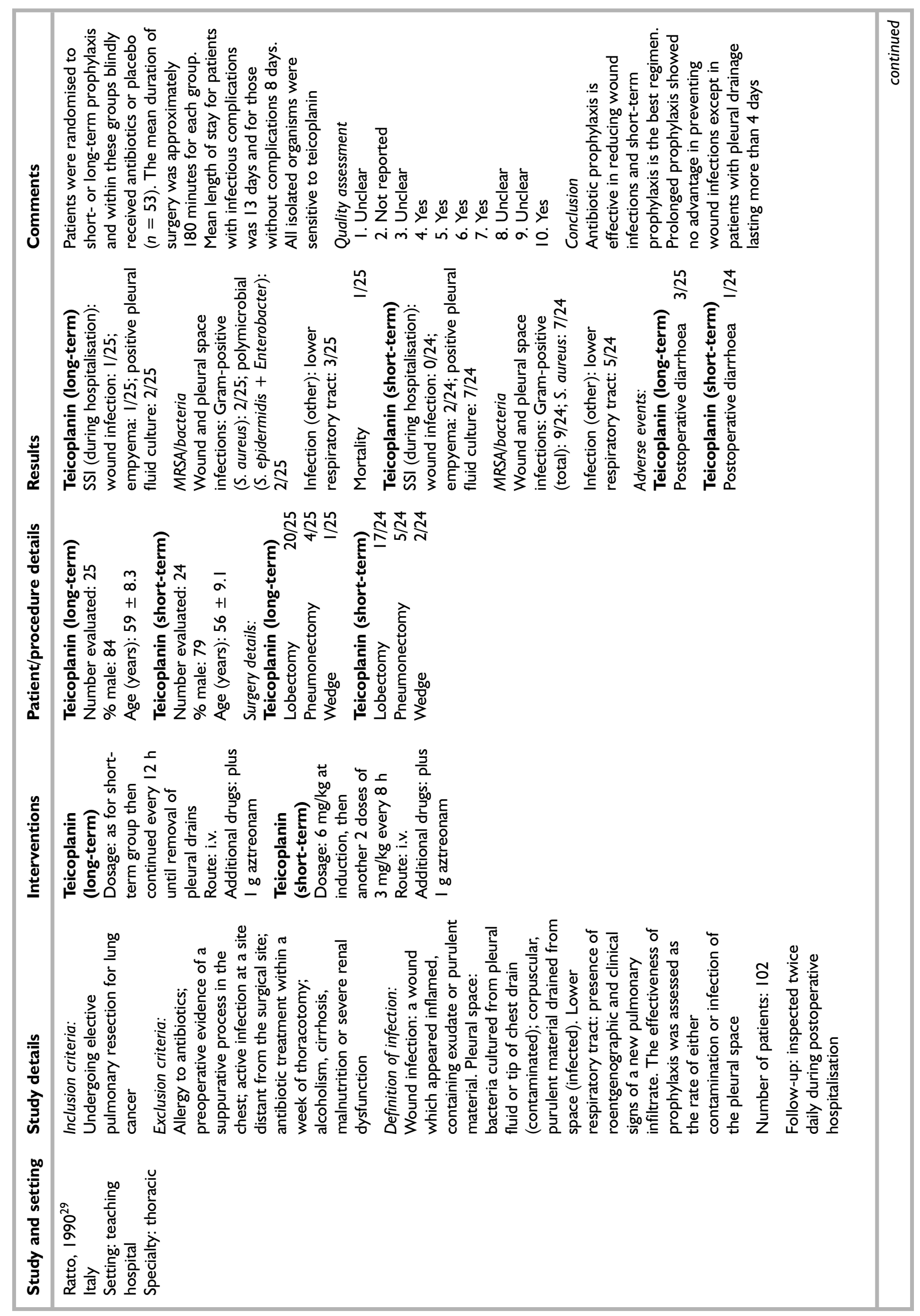




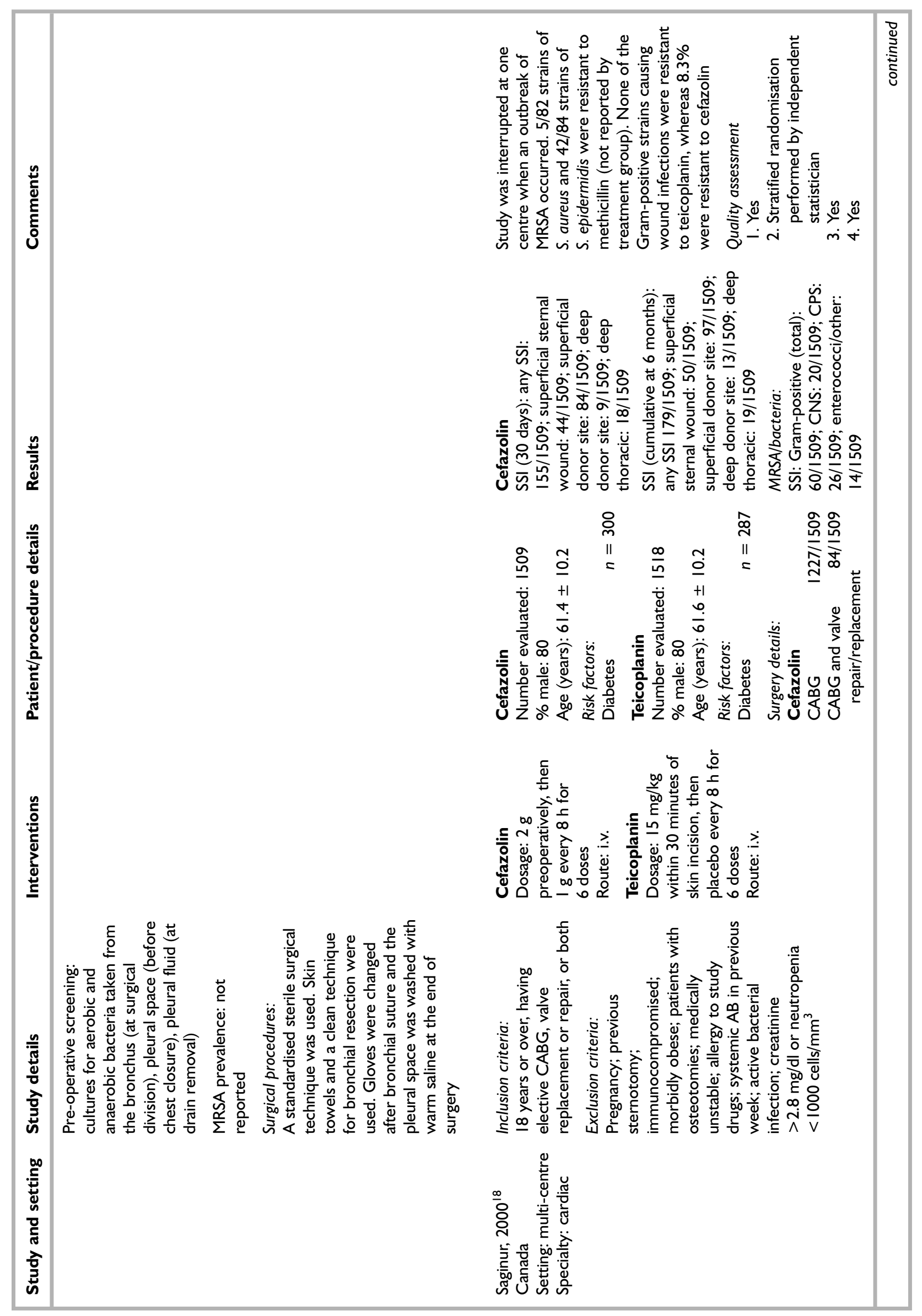




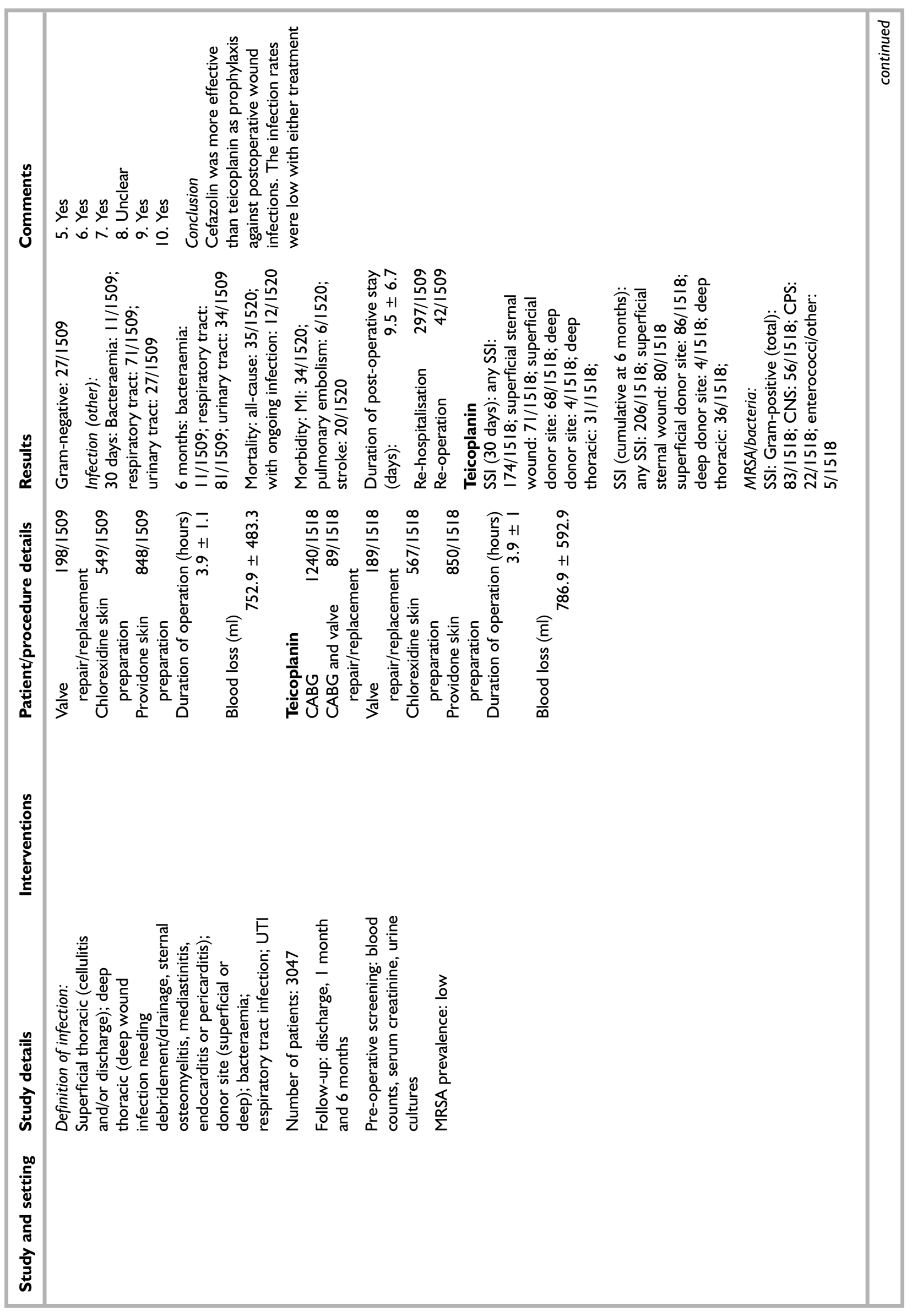




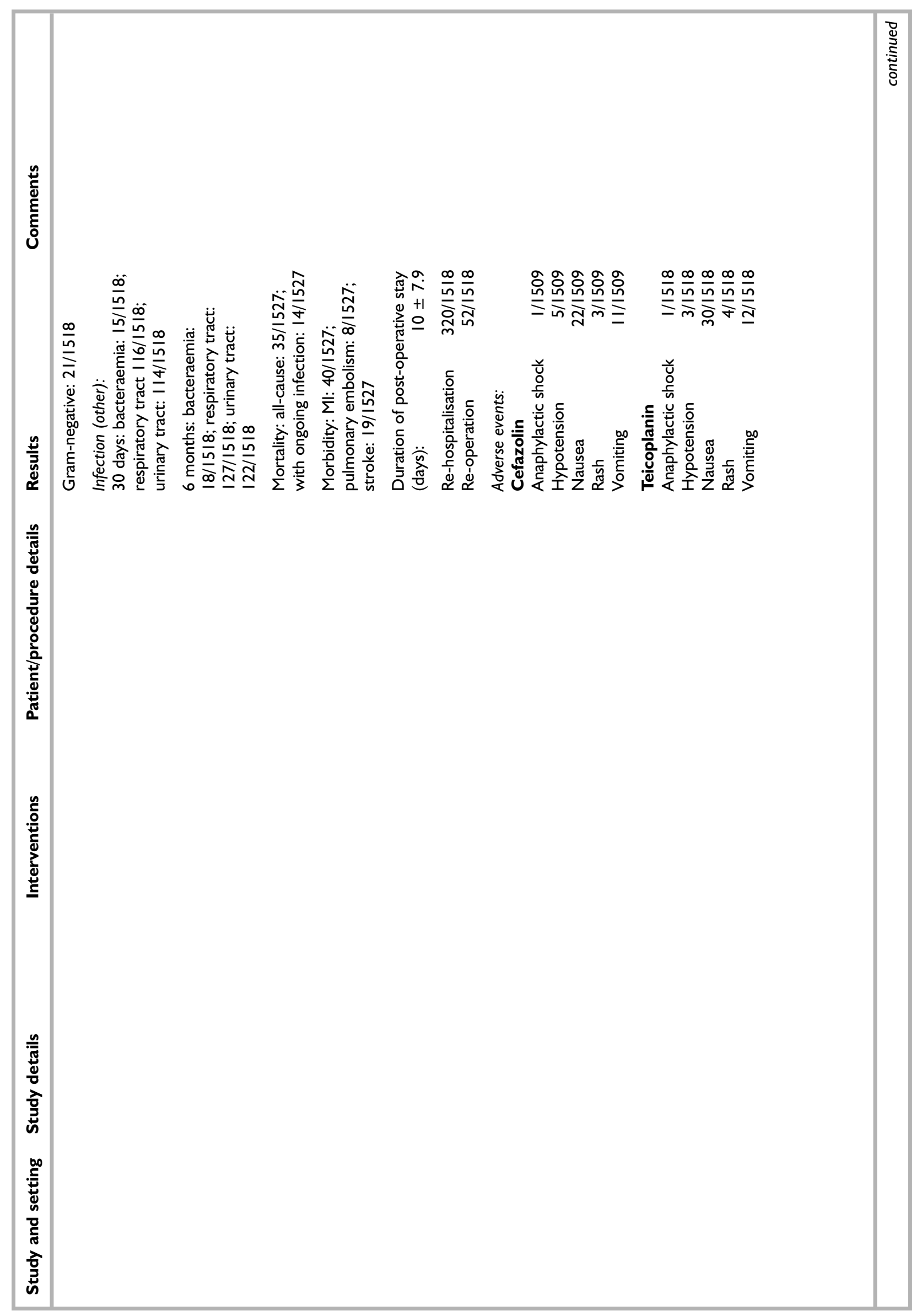




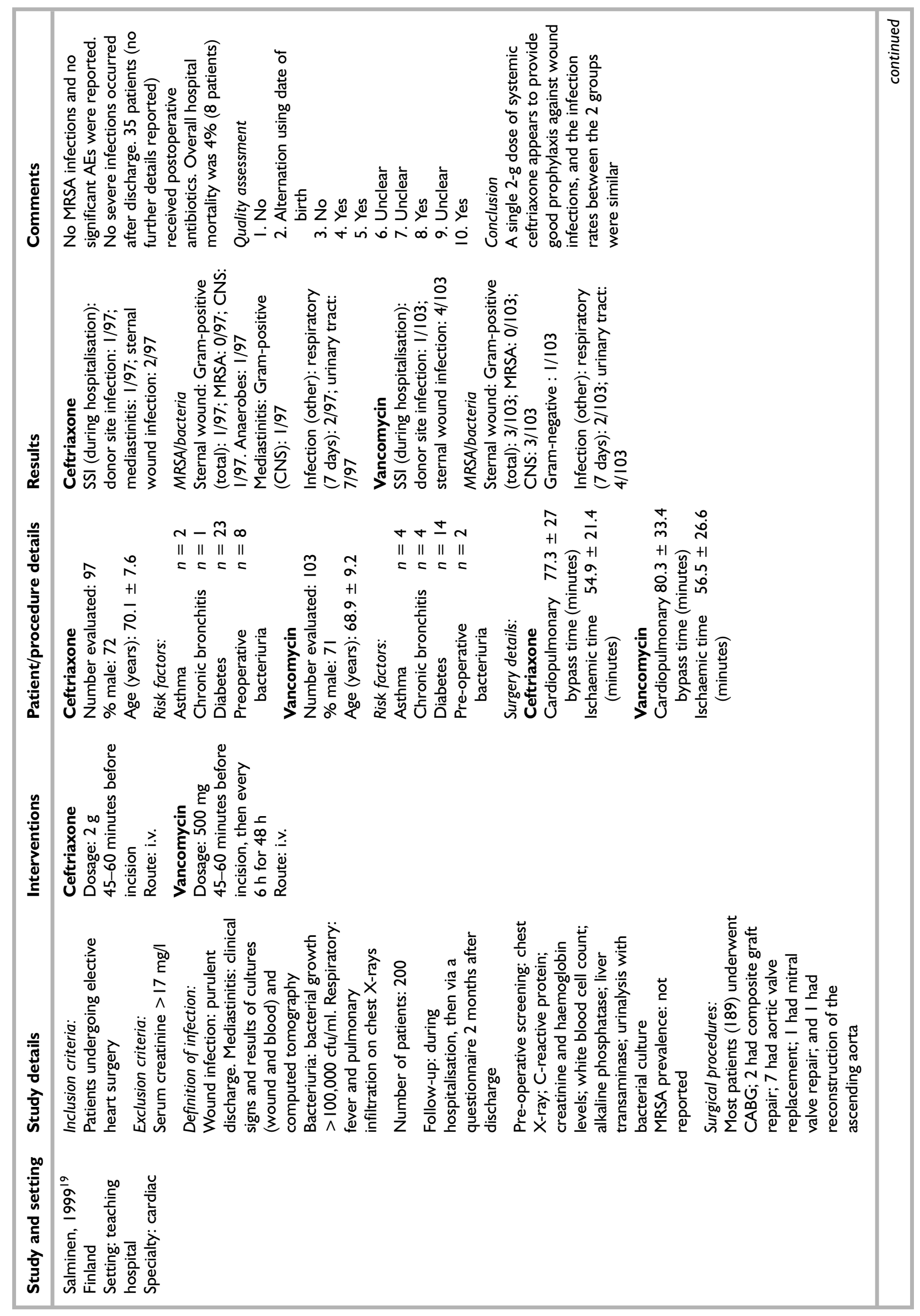




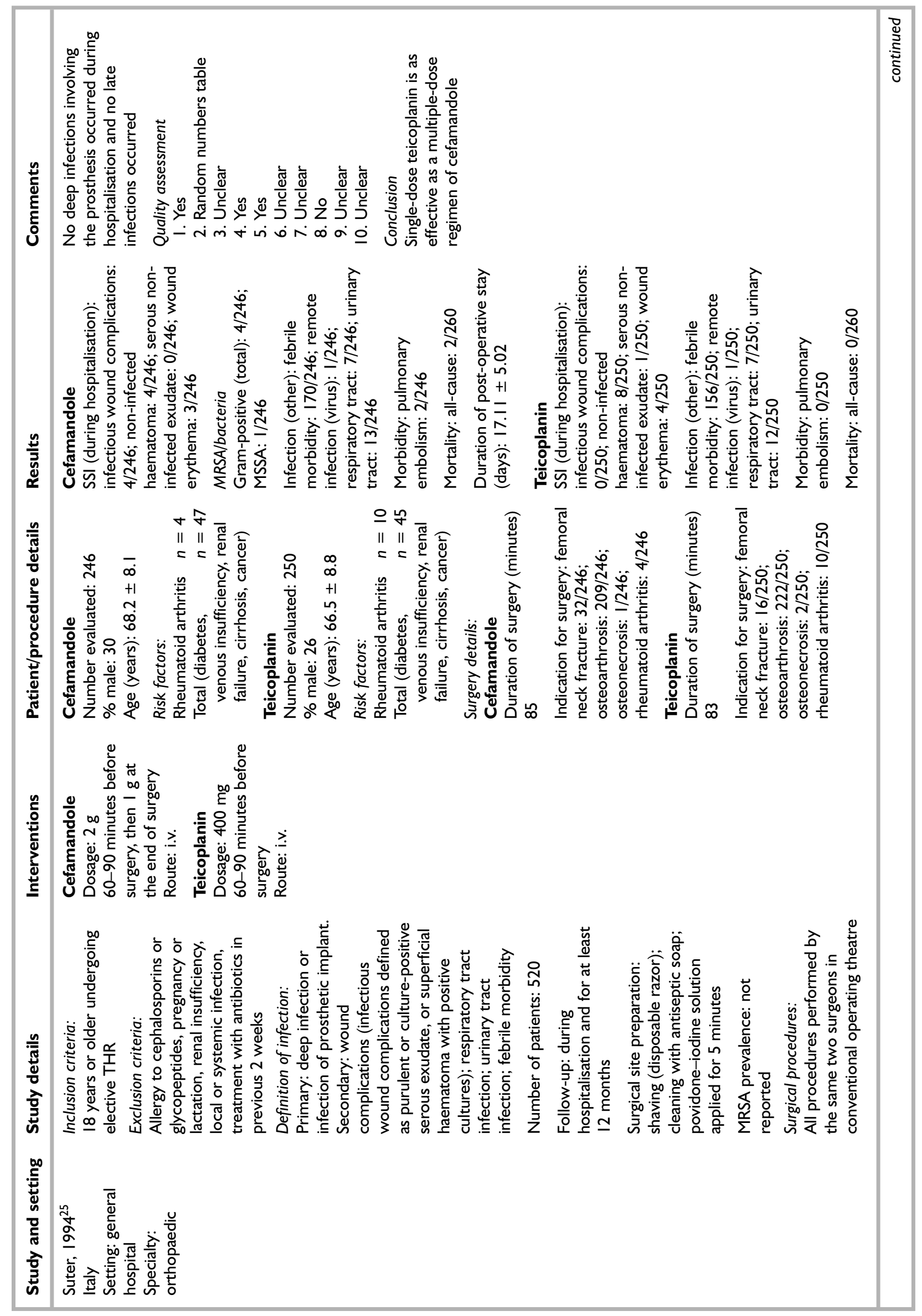




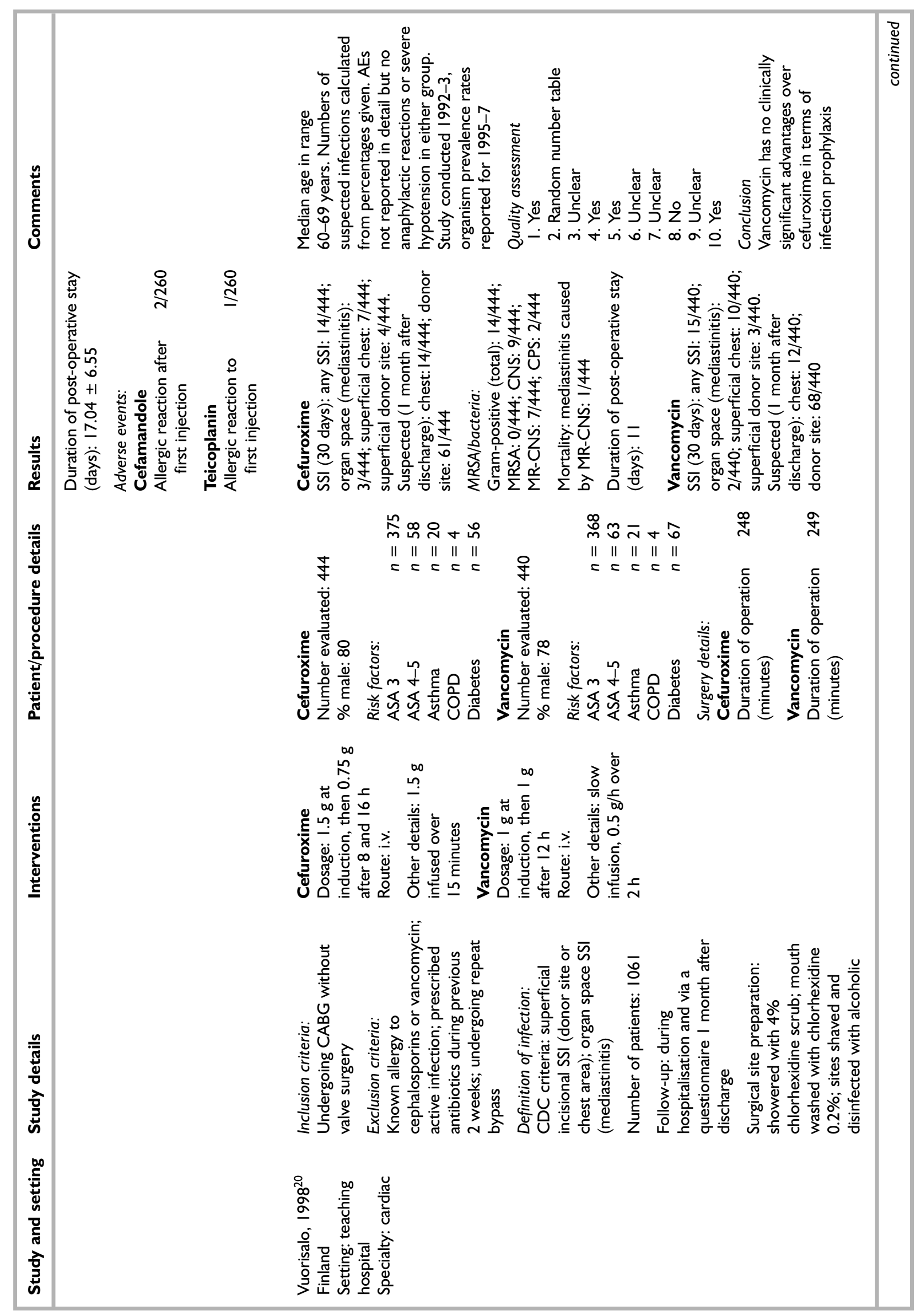




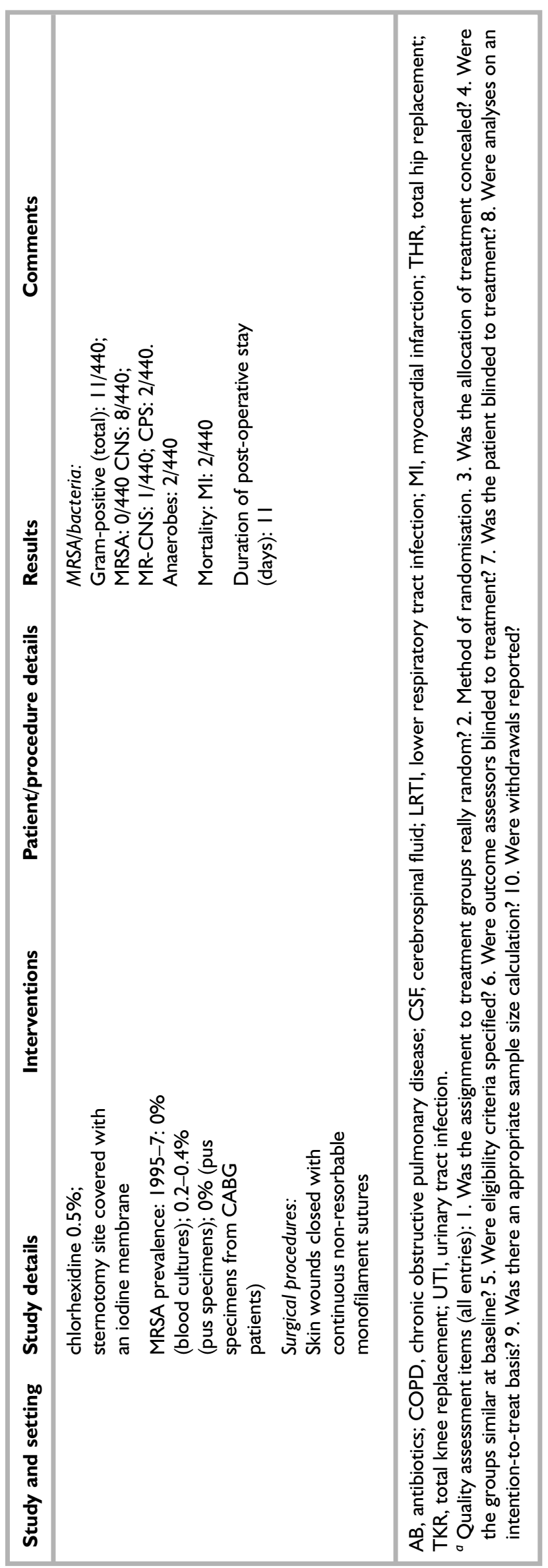




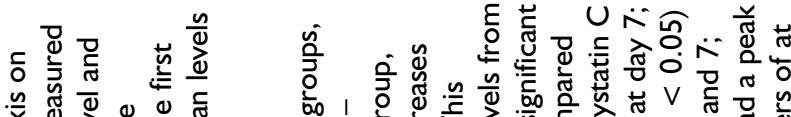

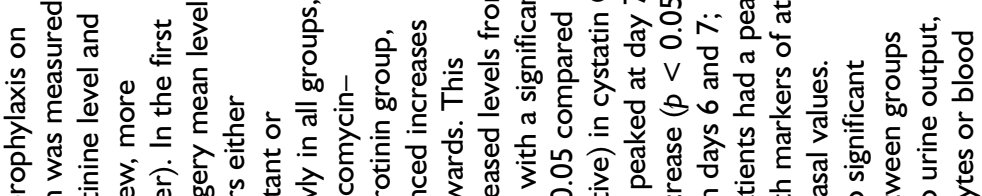

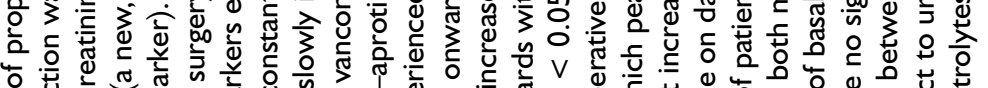

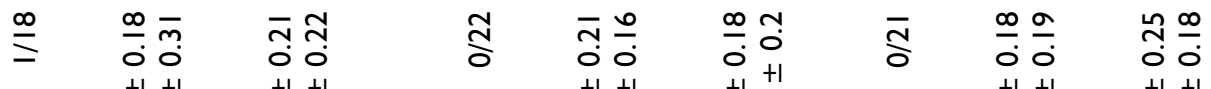

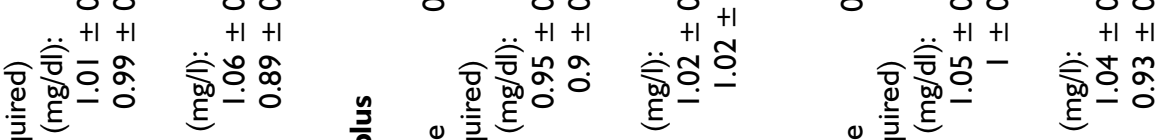

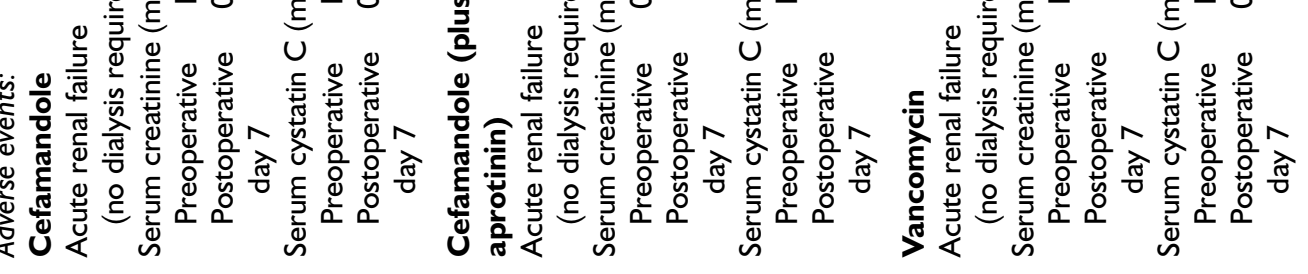

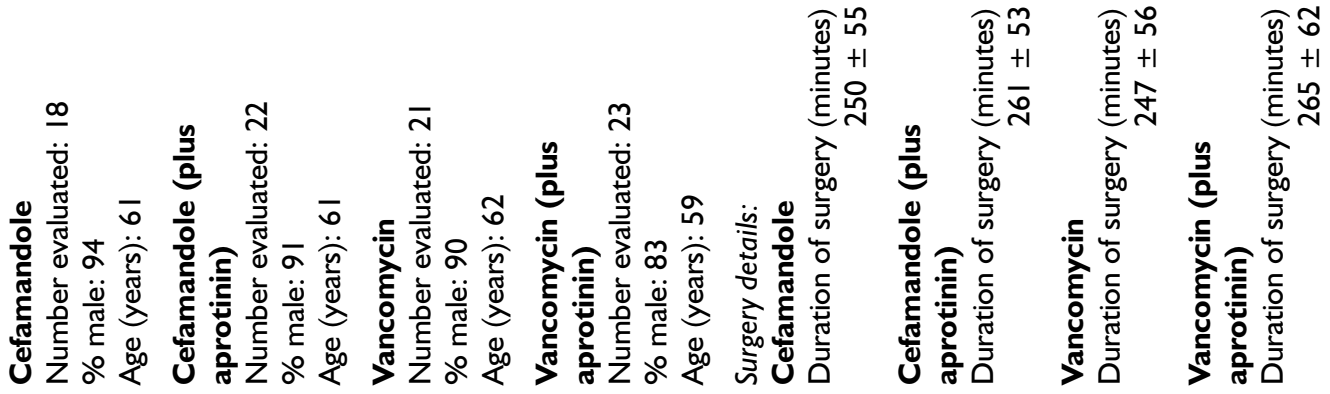

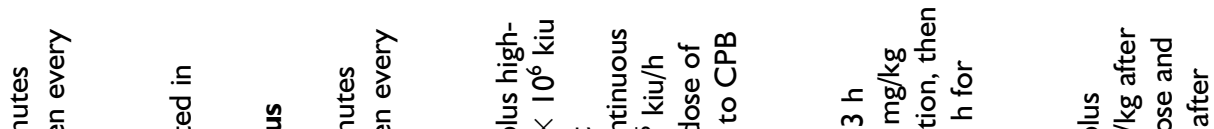

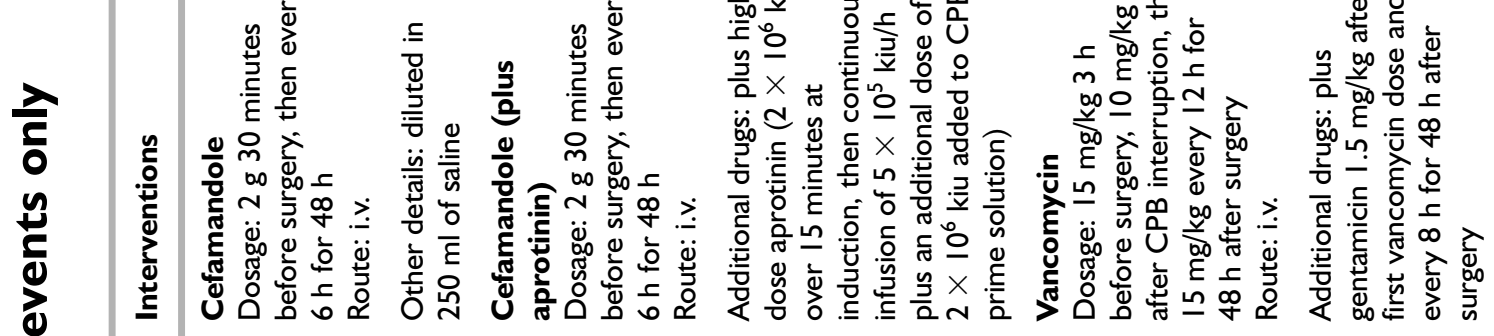

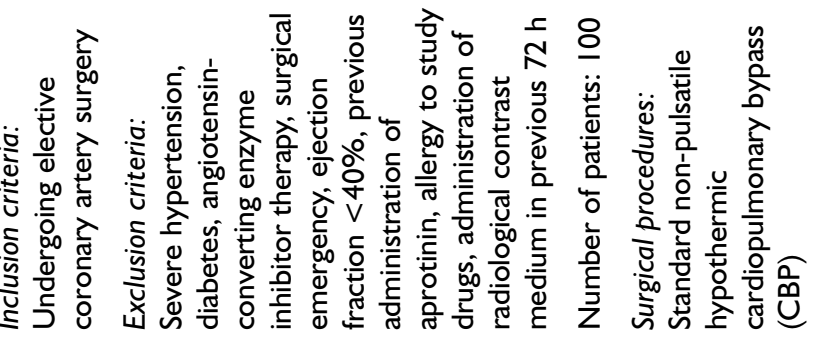




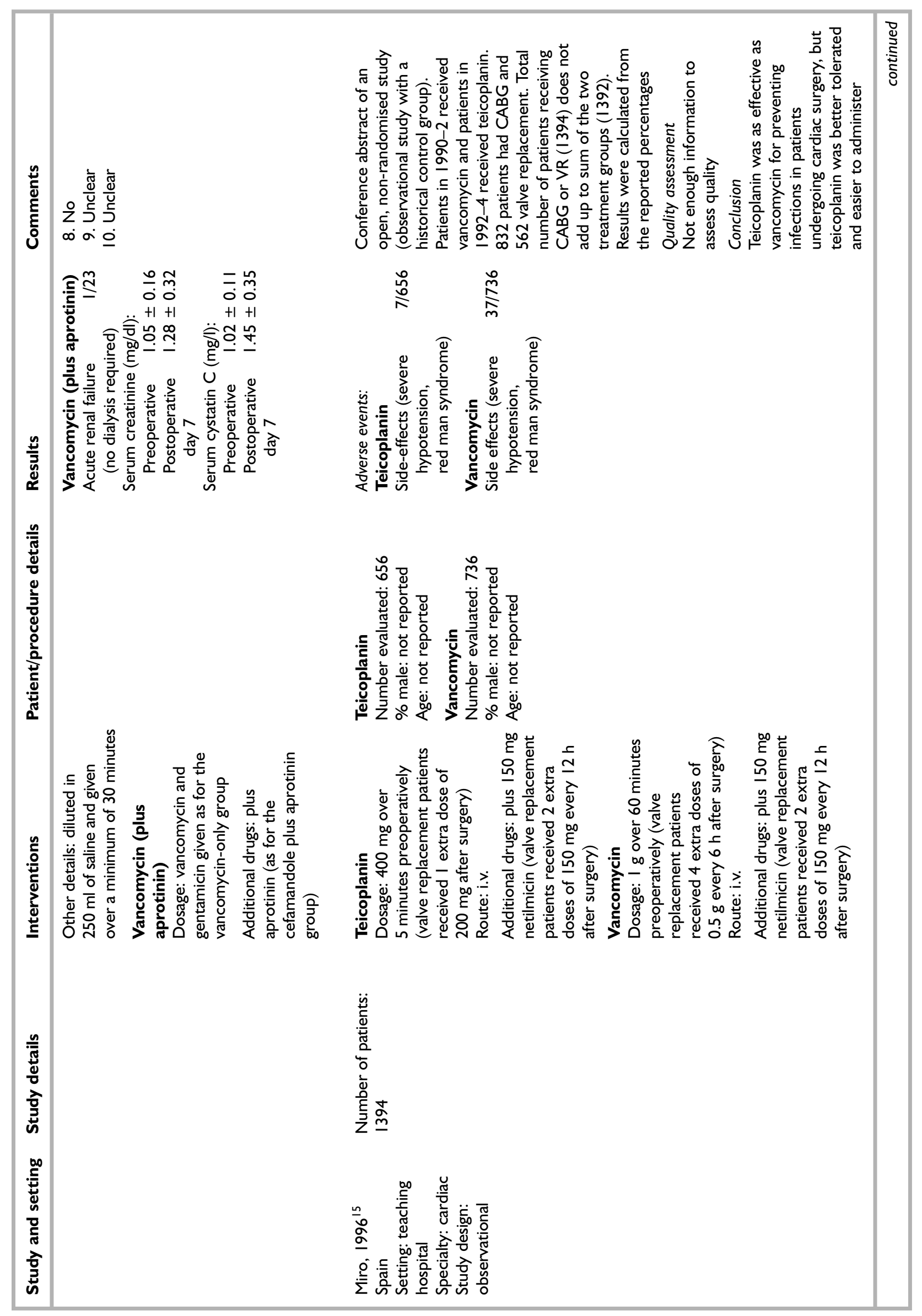




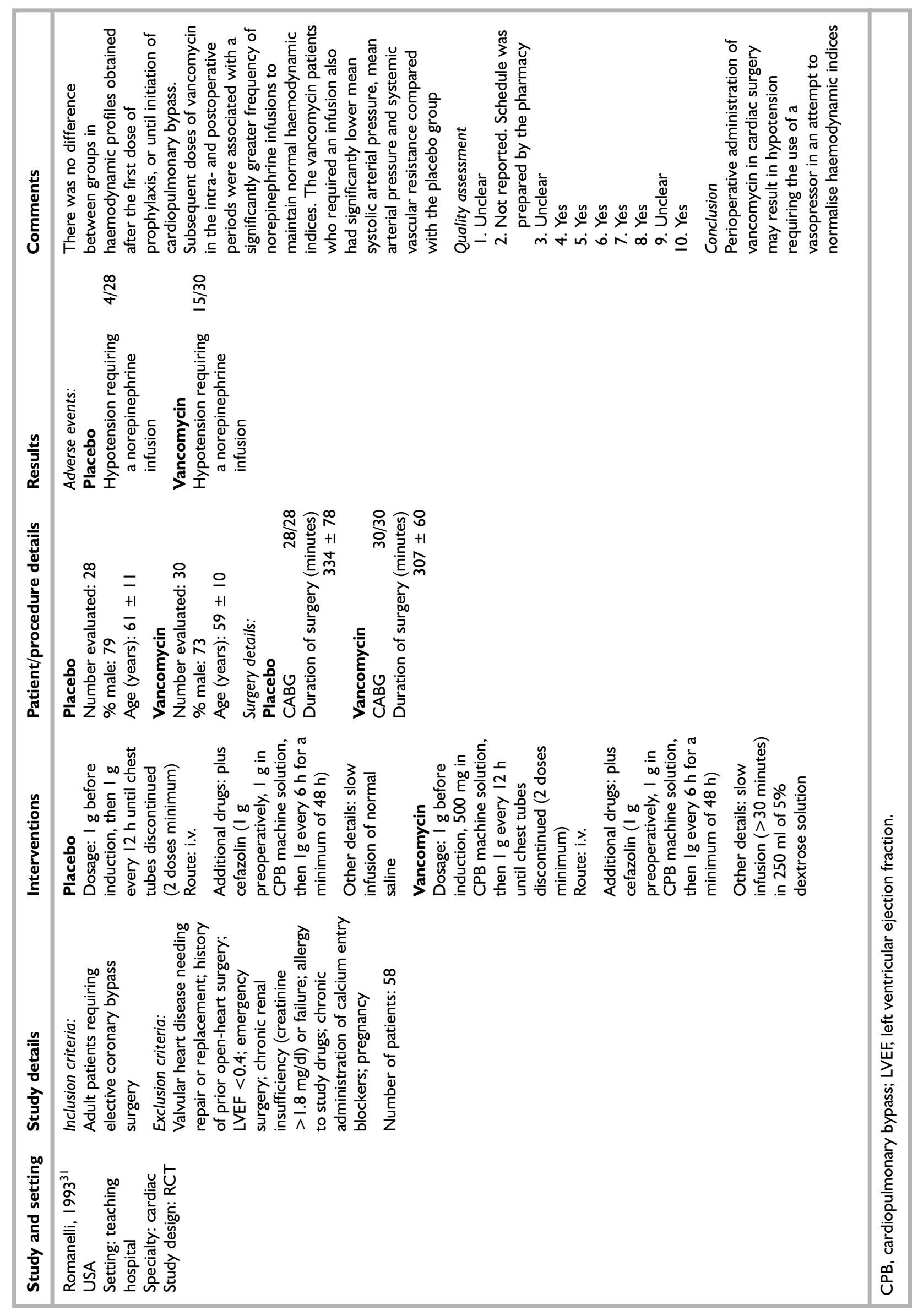




\section{Appendix 5}

\section{Data extraction tables: economic review}

\author{
Study, date of publication \\ Type of economic evaluation \\ Currency used, year \\ Study design \\ Perspective \\ Participants
}

Screening for colonisation/ infection - diagnostic test details and results

Setting, country of study

Type of surgery

Surgical site

Surgical environment

Intervention group intervention

Method of administration of intervention (how administered, when, how long)

Other interventions to reduce infection rates in the intervention group

Control I group intervention

Method of administration of control I intervention

Other interventions to reduce infection rates in the control I group

Resources used

Source of effectiveness data

Length of follow-up

Source of resource use data

Source of unit cost data

Link between cost and effectiveness data

Clinical outcomes measured and methods of valuation used

\section{Codina, $2000^{13}$}

Cost-minimisation analysis

Spanish pesetas, 1998

RCT, prospective single-centre double-blind parallel group

Hospital

Age: teicoplanin group mean age 64 (years) $(S D \pm 11$ ), vancomycin group 64 years $(S D \pm 12)$

Gender: teicoplanin group male/female 167/83, vancomycin group 167/83

Ethnicity: not stated

Diagnosis: patients about to undergo elective cardiac surgery: either VR $(n=233)$ or CABG $(n=267)$

Exclusion criteria: allergy to glycopeptides or netilmicin; active infection; treatment with antibiotic in 5 days prior to surgery; renal insufficiency

No

\section{Hospital, Spain}

Clean

Cardiac

Not stated

Vancomycin ( $n=250$ : CABG arm $n=132$, VR arm $n=118$ )

Single i.v. I-g dose at the induction of anaesthesia

Patients undergoing VR received a second dose at the end of extracorporeal circulation

Also received netilmicin $150 \mathrm{mg}$ and teicoplanin placebo

Teicoplanin ( $n=250$ : CABG arm $n=135$, VR arm $n=115)$

Single i.v. $400 \mathrm{mg}$ dose at the induction of anaesthesia

Patients undergoing VR received a second dose (200 mg teicoplanin) at the end of extracorporeal circulation

Also received netilmicin $150 \mathrm{mg}$ and vancomycin placebo

Drug use, the intravenous mix and the administration costs, personnel input, capital and overheads

Single study

Surgery period

Single study

Hospital costs

Prospective/concurrent

All postoperative infections, based on CDC criteria 


\section{Exploration of antimicrobial resistance, including methods used}

Outcome results/adverse drug events

Cost data handled appropriately

\section{Cost results}

\section{Subgroup analysis \\ Modelling summary}

Outcome measures used in the economic evaluation

Direction of result with appropriate quadrant location

Statistical analysis for patientlevel stochastic data

Appropriateness of statistical analysis

Uncertainty around costeffectiveness expressed and appropriateness of method of dealing with uncertainty around this

Sensitivity analysis and appropriateness

Modelling inputs and techniques appropriate

\section{Authors' conclusions}

Implications for practice

Comments
No

Total number of patients with an adverse drug event at first dose; vancomycin $n=5 \mathrm{I} / 250$, teicoplanin $n=4 / 250$

Total number of patients with an AE (VR second dose; vancomycin $n=6 / 118$, teicoplanin $n=1 / 115$

Severe hypotension; vancomycin $n=1 / 250$, teicoplanin $n=I / 250$

Costs were reported separately from resource use. The source of the unit cost data was reported. Discounting was not relevant

For the CABG patients, when the antibiotics were administered in the surgical room, the cost was 12,005 pts (1998 prices) for those who received vancomycin and 8265 pts for those who received teicoplanin. For the VP patients, when the antibiotics were administered in the surgical room, the cost was 14,528 pts for those who received vancomycin and II,66I pts for those who received teicoplanin. When the antibiotics were administered in a medical ward setting, for the CABG patients, the cost was 2809 pts for those who received vancomycin and 6740 pts for those who received teicoplanin and for the VR patients, the cost was 10, I 40 pts for those who received vancomycin and 5308 pts for teicoplanin

Two patient groups were considered, VR and CABG

Decision tree analysis to calculate the costs associated with prophylaxis for the two patient groups

Severe AEs

Partial economic evaluation

Not undertaken

Not undertaken

Not undertaken

Antibiotic administration in (I) the surgical theatre and (2) the medical ward. Due to the impact of staff costs and different resource use associated with administering either antibiotic, the use of teicoplanin was cheaper if administered in the surgical area whereas the use of vancomycin was cheaper if administered in the medical ward

Two simple decision trees were developed, one to evaluate the impact of the 2 interventions for VR patients, the other to evaluate the impact of the 2 interventions for CABG patients. Analyses were undertaken for medical room and surgery room administration of the drugs

Outcomes were assumed to be the same across groups in terms of severe AEs. For the CABG patients, when the antibiotics were administered in the surgical room, the cost was 12,005 pts (1998 prices) for those who received vancomycin and 8265 pts for those who received teicoplanin. For the VR patients, when the antibiotics were administered in the surgical room, the cost was 14,528 pts for those who received vancomycin and II,66I pts for those who received teicoplanin. When the antibiotics were administered in a medical ward setting, for the CABG patients, the cost was 2809 pts for those who received vancomycin and 6740 pts for those who received teicoplanin and for the VR patients, the cost was 10,140 pts for those who received vancomycin and 5308 pts for teicoplanin

The costs of antibiotic prophylaxis among cardiac surgery patients depend heavily on the setting and the circumstances of drug administration. Teicoplanin was the least costly option when administered in the surgical theatre

No data on MRSA. Resistance issue not considered 


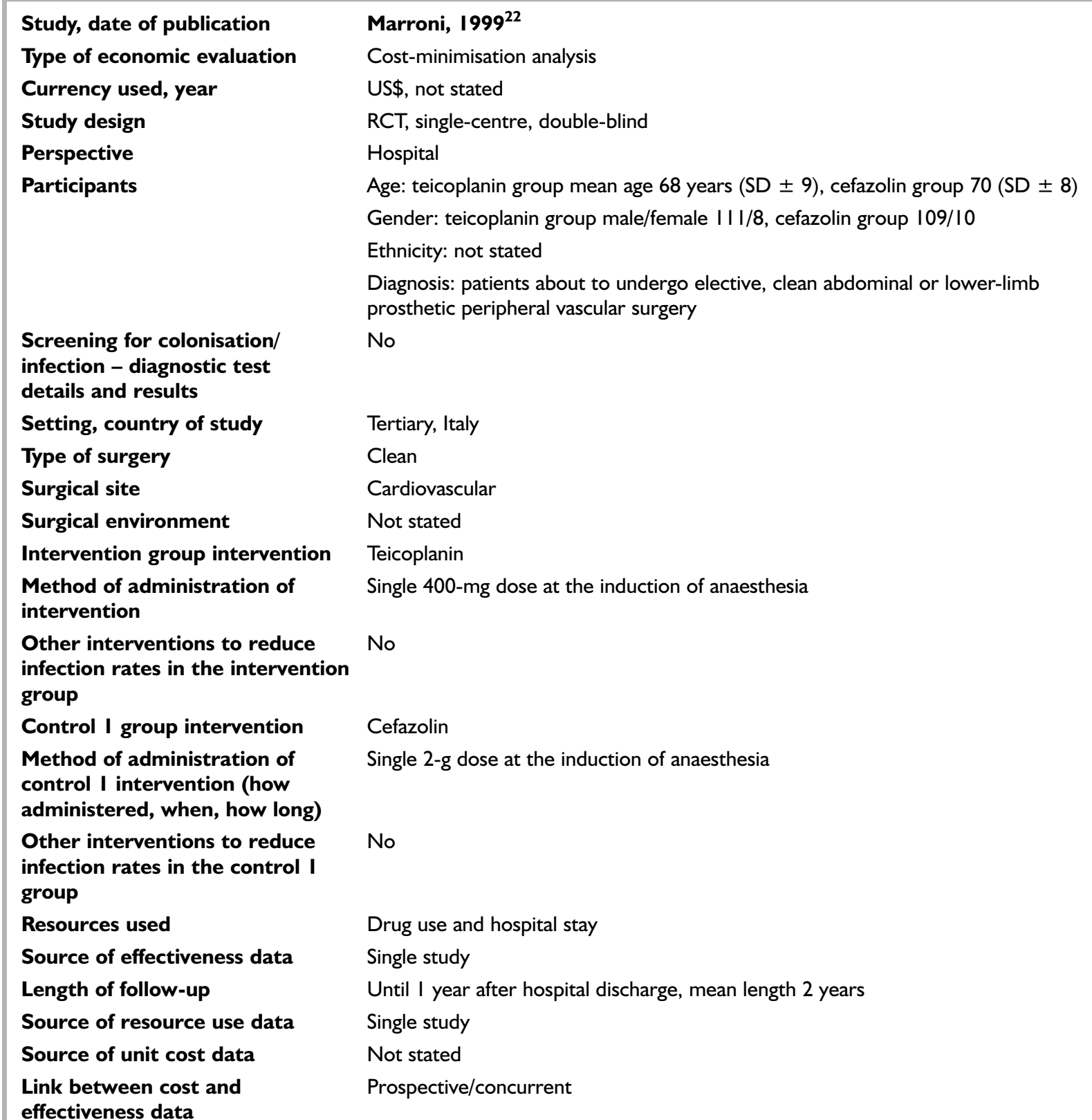

Clinical outcomes measured and Rate of prosthetic and wound infections, overall mortality rate and side-effects methods of valuation used

Exploration of antimicrobial resistance, including methods used

Outcome results/adverse drug events

No

$5.9 \%(n=7)$ of the patients in the teicoplanin group developed SSI whereas $1.7 \%$ $(n=2)$ patients in the cefazolin group developed infections. I patient had MSSA but was cured and had no sign of infection 2 years later.

Early superficial wound infections, diagnosed a mean of 9 days post-operatively, occurred in 5 teicoplanin and 2 cefazolin patients.

Other postoperative infections occurring postoperatively during the hospital stay included in 12 teicoplanin and 14 cefazolin patients.

Mortality rates were similar, $4(3.4 \%)$ in the teicoplanin group and $3(2.5 \%)$ in the cefazolin group. Infective deaths amounted to I person in each group. No sideeffects were observed in either group 
Cost data handled appropriately

\section{Cost results}

\section{Subgroup analysis}

Modelling summary

Outcome measures used in the economic evaluation

\section{Direction of result with} appropriate quadrant location Statistical analysis for patientlevel stochastic data

Appropriateness of statistical analysis

Uncertainty around costeffectiveness expressed and appropriateness of method of dealing with uncertainty around this

Sensitivity analysis and appropriateness

Modelling inputs and techniques appropriate

Authors' conclusions

Implications for practice

\section{Comments}

\section{Study, date of publication}

Type of economic evaluation

Currency used, year

Study design

Perspective

Participants

Screening for colonisation/ infection - diagnostic test details and results

\section{Setting, country of study \\ Type of surgery \\ Surgical site}

\section{Surgical environment}

The cost of drug acquisition and hospital stay was calculated. Costs were reported separately from resource use. The source of the unit cost data was not reported. Discounting was not relevant

The total cost for the teicoplanin group was US\$52,510 higher than that for the cefazolin group

No

Not undertaken

Since the clinical effectiveness analysis demonstrated that there were no significant differences between the two interventions, the economic analysis was based on costs only

Partial evaluation. Authors stated that since there was no statistically significant difference in the effects, it was appropriate to compare costs only

Not undertaken

Not undertaken

Not undertaken

Not undertaken

Not undertaken

Teicoplanin is less toxic and requires less administration. No significant differences in infection rates but teicoplanin $=7(n=5.9 \%)$ patients develop infections, cefazolin $=2(n=1.7 \%)$. Mortality rates, teicoplanin $=4(3.4 \%)$, refazolin $=3(2.5 \%)$, no side-effects observed in either group. As no statistically significant differences in effects, did a cost-minimisation analysis. Cumulative total cost for teicoplanin = US\$571,572 vs cefazolin $=$ US $\$ 519,062$. Drug and hospital stay costs were higher for teicoplanin. Cefazolin was more cost-effective. Cefazolin should remain the most appropriate choice for prophylaxis in patients undergoing vascular surgical procedures The transferability of the results was not discussed

No data on MRSA. Resistance issue not considered

\section{Phillips, 2000 35}

Cost-effectiveness analysis

Canadian \$, price year not reported

Synthesis of published papers

Hospital

Age: not stated

Gender: not stated

Ethnicity: not stated

Diagnosis: hypothetical cohort of cardiovascular surgery patients who were labelled penicillin allergic

No

Tertiary, Canada

Clean/clean contaminated/dirty

Cardiothoracic, vascular, gastrointestinal, orthopaedic, obstetric and gynaecological, head and neck, neurosurgery, urology, general surgery

Clean air, standard, etc. 


\begin{abstract}
Intervention group intervention
Method of administration of intervention (how administered, when, how long)

Other interventions to reduce infection rates in the intervention group

Control I group intervention

Method of administration of control I intervention (how administered, when, how long)

Other interventions to reduce infection rates in the control I group
\end{abstract}

Control 2 group intervention

Method of administration of control 2 intervention (how administered, when, how long)

Other interventions to reduce infection rates in the control 2 group

Control 3 group intervention

Method of administration of control 3 intervention (how administered, when, how long)

Other interventions to reduce infection rates in the control 3 group

Control 4 group intervention

Method of administration of control 4 intervention (how administered, when, how long)

Other interventions to reduce infection rates in the control 4 group

Control 5 group intervention

Method of administration of control 5 intervention (how administered, when, how long)

Other interventions to reduce infection rates in the control 5 group

Resources used

Type of study

Source of effectiveness data

Source of adverse drug events Length of follow-up
Vancomycin to all patients labelled penicillin allergic

Not stated

Not stated

Cefazolin to all patients labelled penicillin allergic

Not stated

Not stated

Obtain a history from all patients labelled penicillin allergic and give vancomycin to all patients with a history suggesting an lgE-mediated reaction to penicillin, and cefazolin to all patients without a history of IgE-mediated reaction

Not stated

Not stated

Administer penicillin skin test to patients with a history suggesting an lgE-mediated reaction to penicillin and give vancomycin to patients with a positive skin test and cefazolin to all others

Not stated

Not stated

Administer a penicillin test to all patients labelled penicillin allergic and then give vancomycin to patients with a positive skin test and cefazolin to all patients with a negative skin test, regardless of history

Not stated

Not stated

Administer a penicillin skin test to all patients labelled penicillin allergic then give vancomycin to patients with either a positive skin test or a history suggesting an lgEmediated reaction to penicillin and give Cefazolin to all others

Not stated

Not stated

Drug use, cephalosporin skin test, treatment of serious reaction to drugs

Decision analytic model

Based on previously published studies

Based on previously published studies

Not reported 
Source of resource use data

Source of unit cost data

Link between cost and

effectiveness data

Clinical outcomes measured and methods of valuation used

Exploration of antimicrobial resistance, including methods used

Outcome results/adverse drug events

Cost data handled appropriately

\section{Cost results}

Subgroup analysis

Modelling summary

Outcome measures used in the economic evaluation

Direction of result with appropriate quadrant location

Statistical analysis for patientlevel stochastic data

Appropriateness of statistical analysis

Uncertainty around costeffectiveness expressed and appropriateness of method of dealing with uncertainty around this

Sensitivity analysis and appropriateness

Modelling inputs and techniques appropriate

\section{Not reported}

Based on actual data provided by Sunnybrook, a tertiary care centre Retrospective

History suggesting an lgE-mediated reaction to penicillin (population labelled penicillin allergic), positive penicillin skin test (population labelled penicillin allergic with history suggesting IgE-mediated reaction to penicillin), serious non-lifethreatening reaction to cefazolin (general population and population labelled penicillin allergic with negative penicillin test), serious non-life-threatening reaction to vancomycin, anaphylaxis to cefazolin (general population and population labelled penicillin allergic with negative penicillin skin test, anaphylaxis to vancomycin (general population)

No

History suggesting an lgE-mediated reaction to penicillin (population labelled penicillin allergic) was 0.42

For positive penicillin skin test (population labelled penicillin allergic with history suggesting lgE-mediated reaction to penicillin 0.14 (range $0.10-0.18$ )

Serious non-life-threatening reaction to cefazolin for general population 0.005 (range $0.005-0.029)$ for population labelled penicillin allergic with negative penicillin test 0.03 (range 0-0.03)

Anaphylaxis to vancomycin (general population) 0.0002 (range 0-0.03)

Unit cost details were reported but details on resource use were not provided.

Discounting was not undertaken. The time frame of the study was not reported but is likely to have been too short to require discounting

No costs were explicitly reported

No

Considering the rate of serious non-life-threatening reactions, strategy 2 dominated strategies $I$ and 3 . The incremental cost per reaction avoided with strategy 4 was Can $\$ 5426$. With strategy 5 the ICER was Can $\$ 10,024$. With strategy 6 the ICER was Can\$10,906

Considering anaphylaxis, the incremental cost per reaction avoided was Can\$166,667 with strategy 3, Can\$159,204 with strategy I, Can\$428,57I with strategy 4, Can $\$ 692,308$ with strategy 5 and Can $\$ 544,776$ with strategy 6 when compared with strategy 2

Rate of serious non-life-threatening reactions and the rate of potentially lifethreatening anaphylactic episodes

North-east

No

Not applicable

Not applicable

All model variables were varied in the analysis. The ranges used were derived from the literature regarding probability values. No justification was provided for the variation in costs tested. The type of sensitivity analysis was not specified but is likely to be univariate

Yes 


Authors' conclusions
Implications for practice
Comments
Study, date of publication
Type of economic evaluation
Currency used, year
Study design
Perspective
Participants

Screening for colonisation/ infection - diagnostic test details and results

\section{Setting, country of study \\ Type of surgery \\ Surgical site \\ Surgical environment \\ Intervention and its administration}

Other interventions to reduce infection rates in the intervention group

Control and its administration

Other interventions to reduce infection rates in control

\section{Resources used}

\section{Source of effectiveness data}

Length of follow-up

Source of resource use data Source of unit cost data

Link between cost and effectiveness data

Clinical outcomes measured and methods of valuation used
The decision analytic model indicated that selective use of vancomycin is more costeffective than indiscriminate use of vancomycin for surgical prophylaxis in cardiovascular surgery patients labelled penicillin allergic

The transferability of the results was not discussed

No data on MRSA. Resistance issue not considered

\section{Spelman, $2002^{36}$}

Cost-effectiveness analysis

Australian \$

Single-centred before-and-after study

Hospital

Age: not stated

Gender: not stated

Ethnicity: not stated

Diagnosis: patients undergoing CABG

No

Tertiary hospital, Australia

Cardiac surgery for CABG

Ig of vancomycin administered i.v. and $600 \mathrm{mg}$ of oral rifampicin preoperatively. The oral rifampicin was administered in the ward prior to the operating room. The vancomycin was administered by the anaesthetist on the patient's arrival in the operating room. A second dose of vancomycin was administered $12 \mathrm{~h}$ postoperatively. Each vancomycin dose was infused during approximately I $\mathrm{h}$ Not specified

Ig of cephazolin administered i.v. One dose preoperatively and 3 doses postoperatively

Not specified

Not specified

Before-and-after study, single-centre

12 months before and 12 months after the change in antibiotic prophylaxis used in the hospital

Single study observational data

Hospital

Prospectively

SSI rate after CABG according to causative organism (based on CDC definitions of SSI)

MRSA

MSSA

Skin or enteric flora

No growth or no specimen

Enterobacteriaceae

Other 


\section{Exploration of antimicrobial resistance, including methods used}

\section{Outcome results/adverse drug events}

Cost data handled appropriately Cost results

\section{Subgroup analysis}

Modelling summary

Outcome measures used in the economic evaluation

Direction of result with appropriate quadrant location

Statistical analysis for patientlevel stochastic data

Appropriateness of statistical analysis

Uncertainty around cost-

effectiveness expressed and

appropriateness of method of dealing with uncertainty around this

Sensitivity analysis and appropriateness

Modelling inputs and techniques appropriate

\section{Authors' conclusions}

Implications for practice

\section{Comments}

Study, date of publication

Type of economic evaluation

Currency used, year

Study design
Not undertaken

SSI rate from 10.5 (95\% Cl 8.2 to 13.3) with cephazolin to 4.9 ( $95 \% \mathrm{Cl} 3.2$ to 7.1$)$ with vancomycin and rifampicin infections per 100 procedures

Surgical site infection rate after CABG according to causative organism MRSA cases: $42=$ cephazolin, $0=$ vancomycin and rifampicin

MSSA: 5 = cephazolin, 2 = vancomycin and rifampicin

Skin or enteric flora: $10=$ cephazolin, $10=$ vancomycin and rifampicin

No growth or no specimen: 3 = cephazolin, $4=$ vancomycin and rifampicin

Enterobacteriaceae: 3 = cephazolin, 7 = vancomycin and rifampicin

Other: 0 = cephazolin, 2 = vancomycin and rifampicin

No adverse drug events were reported

Unclear. The source of unit costs and resource use were not specified

The vancomycin and oral rifampicin group were associated with a statistically significant decrease $(p<0.00 \mathrm{I})$ in the SSI rate and this was estimated to result in a saving of Aus $\$ 576,655$ compared with the first 12 months of follow-up when the patients received cephazolin

Not undertaken

Not undertaken

The primary outcome measure in the economic evaluation was the deep sternal wound infection rate

Costs and benefits not synthesised but results suggest that vancomycin and rifampicin is the dominant strategy (south-east quadrant)

Infection rates were compared using a $\chi^{2}$ test. Statistical analysis of costs was not reported

Appropriate. No other tests were reported

Not undertaken

Not undertaken

Not applicable

Antibiotic prophylaxis using a combination of i.v. vancomycin and oral rifampicin for patients undergoing $C A B G$ surgery resulted in a statistically significant difference in the SSI rate $(p<0.001)$ and costs saved of Aus $\$ 576,655$

The transferability of the results was not discussed. Full details of all resources used and unit costs were not provided, which reduces the chance of applying the results to other settings

No data on MRSA. Resistance issue not considered

Zanetti, 2001 ${ }^{37}$

Cost-effectiveness analysis, cost-utility analysis

US\$, 1998

Controlled trials (unclear as to whether randomised)

Base case: study population was a hypothetical cohort of 10,000 patients undergoing CABG surgery

Synthesis of studies using a decision-analytic model 


\section{Perspective}

Participants

Screening for colonisation/ infection - diagnostic test details and results

Setting, country of study

Type of surgery

Surgical site

Surgical environment

Intervention group I

Method of administration

Other interventions to reduce infection rates in the intervention group

Control group I

\section{Method of administration}

Other interventions to reduce infection rates in the intervention group

\section{Control group 2}

Method of administration

Other interventions to reduce infection rates in the intervention group

Resources used

\section{Type of study}

Source of effectiveness data

Source of adverse drug events

Length of follow-up

Source of resource use data

Source of unit cost data

Link between cost and effectiveness data
Reference case: 65 -year-old man undergoing CABG surgery for stable multi-vessel coronary heart disease

A state transition model was used to incorporate the lifetime probability of death, myocardial infarction, angina, or asymptomatic coronary heart disease following CABG surgery to estimate life expectancy, quality-adjusted life expectancy and total lifetime costs

Base case: healthcare payer perspective

Reference case: societal perspective

Age: Reference case 65 years old

Gender: male

Ethnicity: not studied

Diagnosis: stable, multi-vessel coronary heart disease

Not undertaken

Tertiary care, USA

Clean/clean contaminated

Cardiothoracic

Standard

Routine vancomycin as first line perioperative prophylaxis

5 doses of I $\mathrm{g}$ of vancomycin over $48 \mathrm{~h}$

No

Routine cefazolin, reserving vancomycin for those with a history of allergic reaction to $\beta$-lactam antibiotics

6 doses of Ig of cefazolin over $48 \mathrm{~h}$

No

No routine prophylaxis

Not applicable

No

Intervention: vancomycin, staff time for preparation and administration of drug

Control I: cefazolin, staff time for administration of drug

Control 2: not applicable

Multiple sources from the published literature, national databases and author assumption

Published literature

Base case: 3 months postoperatively

Reference case: 5 years

4 published studies were used to provide resource use data associated with SSIs and hospital deaths. The resource use associated with AEs was based on a single study. The resource use associated with 5 years' follow-up were extrapolated from one published study

4 published studies were used to provide cost data associated with SSIs and hospital deaths. The cost associated with AEs was based on a single study. The costs associated with 5 years' follow-up were extrapolated from one published study

Retrospective 
Clinical outcomes measured and methods of valuation used

Exploration of antimicrobial resistance, including methods used

\section{Outcome results/adverse drug events}

\section{Base case}

Number of hospital deaths avoided

Number of deep SSIs avoided (based on CDC definitions)

Number of superficial SSIs avoided

Incidence of superficial SSI 0.08 (range $0.02-0.12$ )

Incidence of deep SSI 0.04 (range 0.0I-0.06)

Causative organisms:

S. aureus 0.25 (range $0.20-0.35$ )

CNS 0.25 (range $0.20-0.35$ )

Enterococci 0.05 (range 0.02-0.15)

Gram-negative bacteria 0.30 (range 0.15-0.50)

RR of SSI caused by susceptible organisms:

Vancomycin vs no prophlyaxis 0.4 (range $0.20-0.80$ )

Cefazolin vs no prophlyaxis 0.4 (range $0.20-0.80$ )

Incidence of SSI due to resistant organisms:

MRSA (\% of all SSI due to $S$. aureus) $0.012(0.40)$ (range 0-0.03)

MR-CNS (\% of all SSI due to CNS) 0.024 (0.80) (range 0-0.03)

VRE (\% of all SSI due to enterococci) 0.003 (0.15) (range 0-0.006)

Incidence of SSI caused by cefazolin-susceptible Gram-negative bacteria (\% of all SSI due to Gram-negative bacteria) 0.01 (0.28) (range 0-0.036)

History of $\beta$-lactams 0.1 ( 0.05 to 0.15$)$

Probability of hospital death:

Deep SSI 0.082 (range 0.0I-0.10)

Antibiotic allergic reaction 0.00002

CABG surgery related events 0.036 (range $0.01-0.10$ )

\section{Reference case}

QALYs: quality weights were derived from the Beaver Dam Health Outcomes Study. Quality-adjusted life expectancy was estimated by applying quality weights to the health states representing death, myocardial infarction, angina, asymptomatic coronary artery disease. The quality weights were obtained from a published study which used time trade-off techniques to elicit utilities

Future benefits were discounted at a rate of $3 \%$

Impact of antimicrobial resistance explored in the sensitivity analysis not in the model

The authors did conduct a simulation exercise to explore an increase in VRE by $2 \%$ per year; however, given the current lack of knowledge about glycopeptide resistance to staphylococci, they were unwilling to place any weight on this

\section{Base case}

Routine vancomycin deep SSI 368 and 388 hospital deaths per 10,000 patients. Compared with no prophylaxis, routine vancomycin resulted in 29 fewer deep SSI and 58 fewer superficial SSI and 3 fewer deaths

Routine cefazolin deep SSI 397 and 39I hospital deaths per 10,000 patients.

Compared with no prophylaxis, routine cefazolin resulted in 173 fewer deep SSI and 347 fewer superficial SSI and 14 fewer deaths

No prophylaxis deep SSI 570 and 405 hospital deaths per 10,000 patients

\section{Reference case}

Routine vancomycin 8.339 QALYs or 0.004 incremental QALYs compared with cefazolin

Routine cefazolin 8.335 QALYs or 0.023 incremental QALYs compared with no prophylaxis

No prophylaxis 8.312 QALYs 


\begin{abstract}
Adverse drug events
Cost data handled appropriately

\section{Cost results}

\section{Subgroup analysis}

Modelling summary

\section{Direction of result with appropriate quadrant location}

Statistical analysis for patientlevel stochastic data

Appropriateness of statistical analysis

Uncertainty around costeffectiveness expressed and appropriateness of method of dealing with uncertainty around this

Sensitivity analysis and appropriateness

Incidence of antibiotic-related AEs

Vancomycin 0.08 (range $0.01-0.20$ ) (adjusted estimate reflecting the probability of toxicity with a 2-day prophylactic regimen by assuming a linear relationship between incidence of AEs and duration of therapy)

Cefazolin 0.08 (range 0.0I-0.20)

Cost data were not reported separately from resource use data. No productivity losses were calculated for the reference case analysis even though the perspective was stated as societal

Future costs were discounted at a rate of $3 \%$

\section{Base case}

Routine vancomycin total cost per 10,000 patients was US $\$ 23,360,000$

This resulted in an incremental saving of US\$I, I 70,000 per 10,000 patients from using cefazolin instead of no antibiotic prophylaxis

Routine cefazolin total cost per 10,000 patients was US $\$ 24,530,000$

No prophylaxis total cost per 10,000 patients was US $\$ 33,410,000$

This resulted in an incremental saving of US $\$ 8,880,000$ per 10,000 patients from using cefazolin instead of no antibiotic prophylaxis

\section{Reference case}

Routine vancomycin total lifetime cost $=$ US $\$ 61,913$

Incremental saving of US\$103 if vancomycin used instead of cefazolin

Routine cefazolin total lifetime cost $=$ US $\$ 62,016$

Incremental saving of US\$876 if cefazolin used instead of no prophylaxis

No

Benefits and costs were not combined as the cefazolin strategy dominated the no prophylaxis strategy and the vancomycin strategy was as effective and cost-saving compared with cefazolin

Costs and benefits not synthesised as the cefazolin strategy dominated the no prophylaxis strategy. The vancomycin strategy was as cost-saving and as effective as the cefazolin strategy, but results suggest that vancomycin and rifampicin is the dominant strategy (south-east quadrant)

No

Costs were treated deterministically

Not undertaken

\section{Base case}

Univariate and multivariate sensitivity analyses were undertaken to determine the impact of variability in the model input parameters. The plausible ranges (above) were used as parameters for testing for deaths from all causes and SSI-related deaths, distribution of causative organisms, incidence of prophylaxis-related AEs, proportion of patients with allergy to $\beta$-lactam antibiotics, costs of cefazolin, deep or superficial SSI, death or prophylaxis-related events. Results were most sensitive to changes in the cost of vancomycin, efficacy of cefazolin and vancomycin in preventing $\mathrm{SSI}$, and prevalence of bacterial resistance to antibiotics

A multi-way sensitivity analysis of the impact of different antibiotic susceptibility profiles was undertaken. Routine vancomycin remained the most effective and least costly strategy, independent of the prevalence of VRE
\end{abstract}


Modelling inputs and techniques appropriate

Authors' conclusions

Implications for practice

Comments

\section{Reference case}

Results were most sensitive to the acquisition and administration cost of vancomycin, the efficacy of vancomycin and cefazolin in preventing SSIs and the prevalence of bacterial resistance to antibiotics

Incidence of SSI due to resistant organisms:

MRSA (\% of all SSI due to S. aureus) $0.012(0.40)$ (range 0-0.03)

MR-CNS (\% of all SSI due to CNS) 0.024 (0.80) (range 0-0.03)

VRE (\% of all SSI due to enterococci) $0.003(0.15)$ (range 0-0.006)

Incidence of SSI caused by cefazolin-susceptible Gram-negative bacteria (\% of all SSI due to Gram-negative bacteria) 0.01 (0.28) (range 0-0.036)

In the sensitivity analysis, the study explored the impact of different antibiotic susceptibility profiles on the results. A three-way sensitivity analysis of the impact of different antibiotic susceptibility profiles, that is, for MRSA, MR-CNS and cefazolinsusceptible Gram-negative bacteria, was undertaken. Routine vancomycin remained the most effective and least costly strategy, independent of the prevalence of VRE

The impact of different patterns of antimicrobial resistance on the ICER associated with vancomycin compared with routine cefazolin was calculated

For the base case analysis a decision analytic model was used and for the reference case a state transition model was used

A strategy of no prophylaxis was always less effective and more costly than using prophylaxis. Use of routine vancomycin prior to CABG is more effective and costeffective than cefazolin prophylaxis and no routine antibiotic prophylaxis prior to surgery

Vancomycin rather than cefazolin should be used for routine prophylaxis prior to clean surgical procedures that would save lives and hospital costs. However, since the issue of resistance was not explored, the authors were reluctant to recommend a change in practice

Resistance issue not considered 


\section{Appendix 6}

\section{Economic evaluation quality assessment}




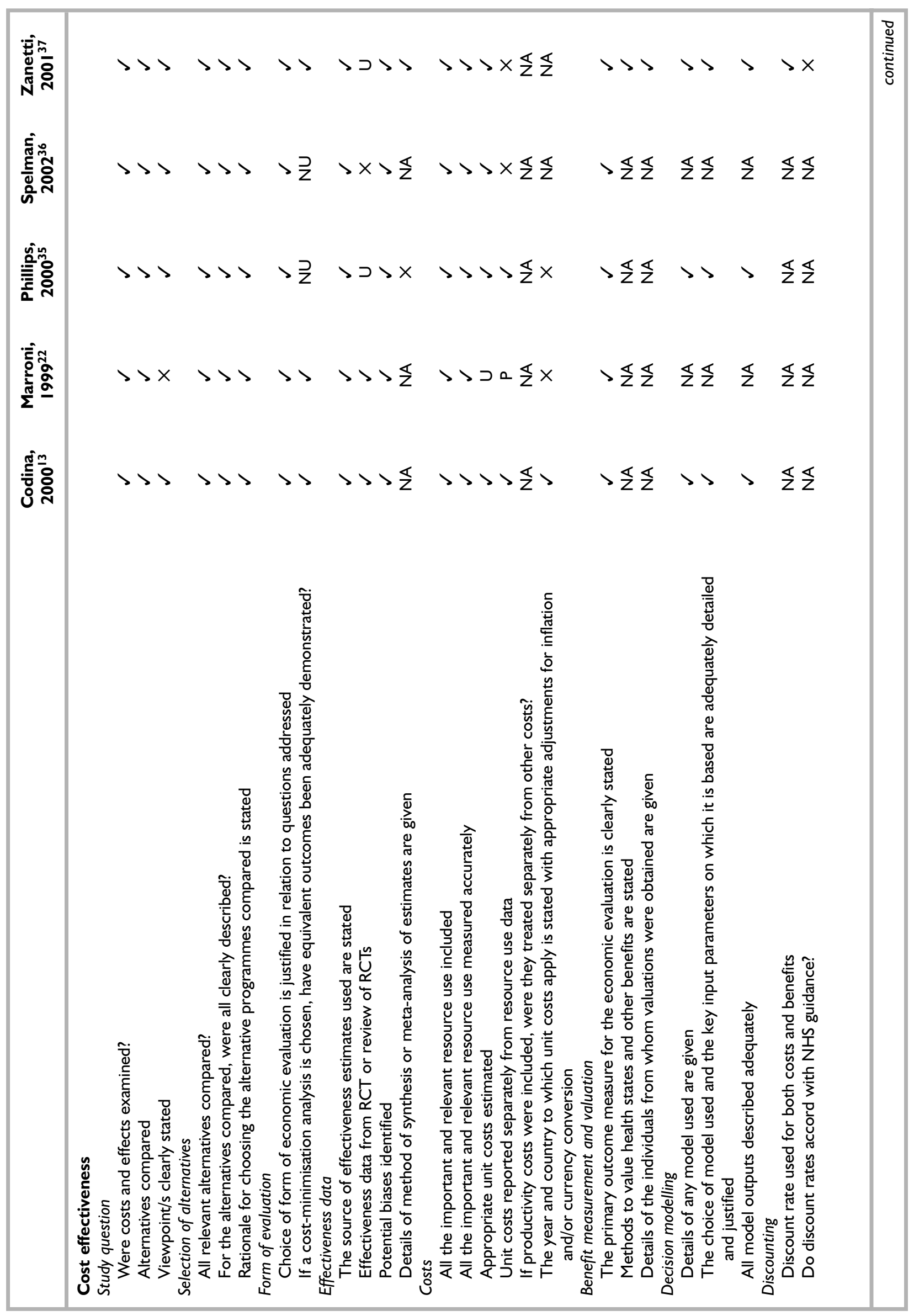




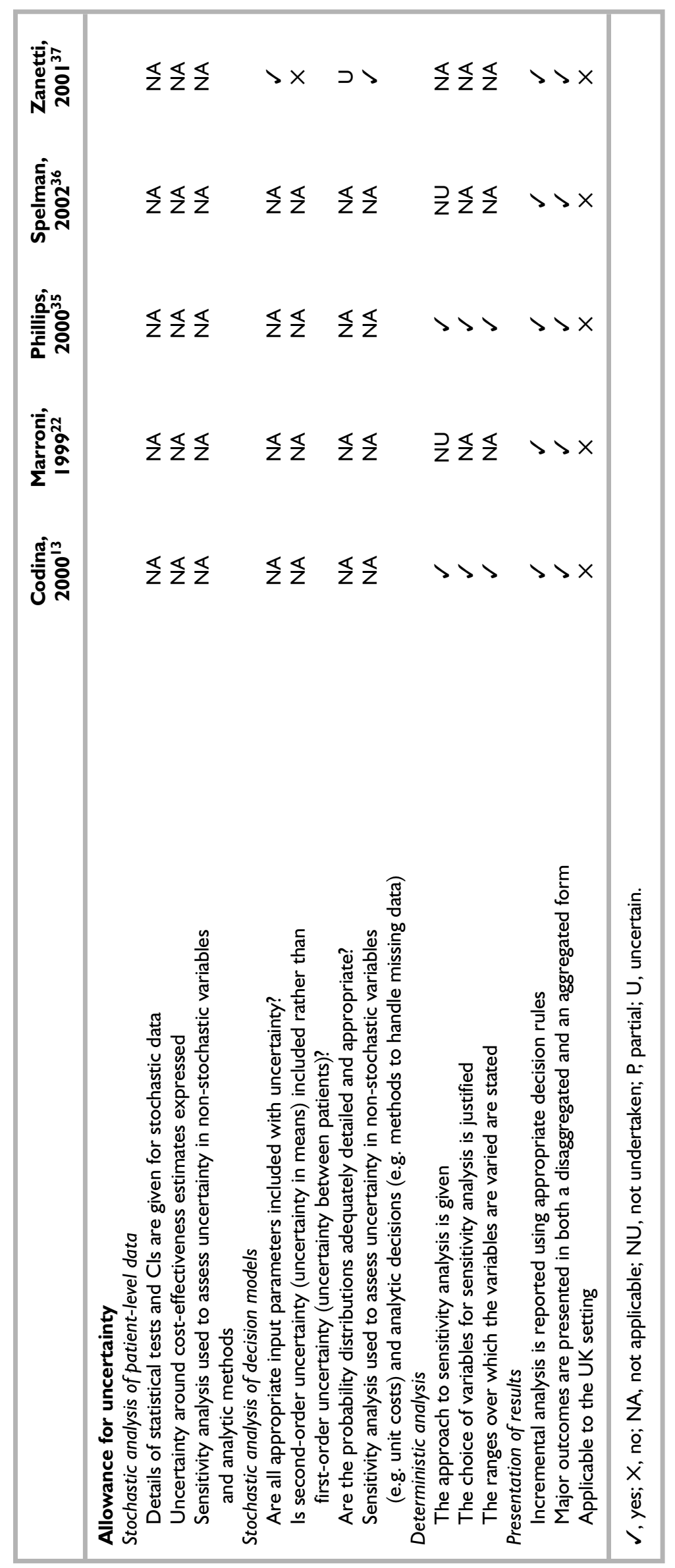





\section{Health Technology Assessment reports published to date}

\section{Volume I, 1997}

No. 1

Home parenteral nutrition: a systematic review.

By Richards DM, Deeks JJ, Sheldon TA, Shaffer JL.

No. 2

Diagnosis, management and screening of early localised prostate cancer.

A review by Selley S, Donovan J, Faulkner A, Coast J, Gillatt D.

No. 3

The diagnosis, management, treatment and costs of prostate cancer in England and Wales.

A review by Chamberlain J, Melia J, Moss S, Brown J.

No. 4

Screening for fragile $\mathrm{X}$ syndrome.

A review by Murray J, Cuckle $\mathrm{H}$, Taylor G, Hewison J.

No. 5

A review of near patient testing in primary care.

By Hobbs FDR, Delaney BC, Fitzmaurice DA, Wilson S, Hyde CJ, Thorpe GH, et al.

No. 6

Systematic review of outpatient services for chronic pain control.

By McQuay HJ, Moore RA, Eccleston C, Morley S, de C Williams AC.

No. 7

Neonatal screening for inborn errors of metabolism: cost, yield and outcome.

A review by Pollitt RJ, Green A,

McCabe CJ, Booth A, Cooper NJ,

Leonard JV, et al.

No. 8

Preschool vision screening.

A review by Snowdon SK, Stewart-Brown SL.

No. 9

Implications of socio-cultural contexts for the ethics of clinical trials.

A review by Ashcroft RE, Chadwick

DW, Clark SRL, Edwards RHT, Frith L, Hutton JL.

No. 10

A critical review of the role of neonatal hearing screening in the detection of congenital hearing impairment.

By Davis A, Bamford J, Wilson I,

Ramkalawan T, Forshaw M, Wright S.

\section{No. 11}

Newborn screening for inborn errors of metabolism: a systematic review.

By Seymour CA, Thomason MJ,

Chalmers RA, Addison GM, Bain MD, Cockburn F, et al.

No. 12

Routine preoperative testing: a systematic review of the evidence. By Munro J, Booth A, Nicholl J.

No. 13

Systematic review of the effectiveness of laxatives in the elderly.

By Petticrew M, Watt I, Sheldon T.

No. 14

When and how to assess fast-changing technologies: a comparative study of medical applications of four generic technologies.

A review by Mowatt G, Bower DJ, Brebner JA, Cairns JA, Grant AM, McKee L.

\section{Volume 2, 1998}

No. 1

Antenatal screening for Down's syndrome.

A review by Wald NJ, Kennard A, Hackshaw A, McGuire A.

No. 2

Screening for ovarian cancer:

a systematic review.

By Bell R, Petticrew M, Luengo S, Sheldon TA.

No. 3

Consensus development methods, and their use in clinical guideline development.

A review by Murphy MK, Black NA, Lamping DL, McKee CM, Sanderson CFB, Askham J, et al.

No. 4

A cost-utility analysis of interferon beta for multiple sclerosis.

By Parkin D, McNamee P, Jacoby A, Miller P, Thomas S, Bates D.

No. 5

Effectiveness and efficiency of methods of dialysis therapy for end-stage renal

disease: systematic reviews.

By MacLeod A, Grant A

Donaldson C, Khan I, Campbell M, Daly C, et al.
No. 6

Effectiveness of hip prostheses in primary total hip replacement: a critical review of evidence and an economic model.

By Faulkner A, Kennedy LG,

Baxter K, Donovan J, Wilkinson M, Bevan G.

\section{No. 7}

Antimicrobial prophylaxis in colorectal surgery: a systematic review of randomised controlled trials. By Song F, Glenny AM.

No. 8

Bone marrow and peripheral blood stem cell transplantation for malignancy.

A review by Johnson PWM, Simnett SJ, Sweetenham JW, Morgan GJ, Stewart LA.

No. 9

Screening for speech and language delay: a systematic review of the literature.

By Law J, Boyle J, Harris F, Harkness A, Nye C.

No. 10

Resource allocation for chronic stable angina: a systematic review of effectiveness, costs and cost-effectiveness of alternative interventions.

By Sculpher MJ, Petticrew M, Kelland JL, Elliott RA, Holdright DR, Buxton MJ.

No. 11

Detection, adherence and control of hypertension for the prevention of stroke: a systematic review.

By Ebrahim S.

No. 12

Postoperative analgesia and vomiting, with special reference to day-case surgery: a systematic review.

By McQuay HJ, Moore RA.

No. 13

Choosing between randomised and nonrandomised studies: a systematic review.

By Britton A, McKee M, Black N, McPherson K, Sanderson C, Bain C.

No. 14

Evaluating patient-based outcome measures for use in clinical trials.

A review by Fitzpatrick R, Davey C, Buxton MJ, Jones DR. 
No. 15

Ethical issues in the design and conduct of randomised controlled trials.

A review by Edwards SJL, Lilford RJ, Braunholtz DA, Jackson JC, Hewison J, Thornton J.

\section{No. 16}

Qualitative research methods in health technology assessment: a review of the literature.

By Murphy E, Dingwall R, Greatbatch D, Parker S, Watson P.

No. 17

The costs and benefits of paramedic skills in pre-hospital trauma care.

By Nicholl J, Hughes S, Dixon S, Turner J, Yates D.

\section{No. 18}

Systematic review of endoscopic ultrasound in gastro-oesophageal cancer.

By Harris KM, Kelly S, Berry E, Hutton J, Roderick P, Cullingworth J, et al.

No. 19

Systematic reviews of trials and other studies.

By Sutton AJ, Abrams KR, Jones DR, Sheldon TA, Song F

No. 20

Primary total hip replacement surgery: a systematic review of outcomes and modelling of cost-effectiveness associated with different prostheses.

A review by Fitzpatrick R, Shortall E, Sculpher M, Murray D, Morris R, Lodge $\mathrm{M}$, et al.

\section{Volume 3, 1999}

No. 1

Informed decision making: an annotated bibliography and systematic review.

By Bekker H, Thornton JG,

Airey CM, Connelly JB, Hewison J, Robinson MB, et al.

No. 2

Handling uncertainty when performing economic evaluation of healthcare interventions.

A review by Briggs AH, Gray AM.

No. 3

The role of expectancies in the placebo effect and their use in the delivery of health care: a systematic review.

By Crow R, Gage H, Hampson S, Hart J, Kimber A, Thomas H.

No. 4

A randomised controlled trial of different approaches to universal antenatal HIV testing: uptake and acceptability. Annex: Antenatal HIV testing - assessment of a routine voluntary approach.

By Simpson WM, Johnstone FD, Boyd FM, Goldberg DJ, Hart GJ, Gormley
No. 5

Methods for evaluating area-wide and organisation-based interventions in health and health care: a systematic review.

By Ukoumunne OC, Gulliford MC, Chinn S, Sterne JAC, Burney PGJ.

No. 6

Assessing the costs of healthcare technologies in clinical trials.

A review by Johnston $\mathrm{K}$, Buxton $\mathrm{MJ}$, Jones DR, Fitzpatrick R.

No. 7

Cooperatives and their primary care emergency centres: organisation and impact.

By Hallam L, Henthorne K.

No. 8

Screening for cystic fibrosis.

A review by Murray J, Cuckle $\mathrm{H}$, Taylor G, Littlewood J, Hewison J.

No. 9

A review of the use of health status measures in economic evaluation.

By Brazier J, Deverill M, Green C, Harper R, Booth A.

No. 10

Methods for the analysis of quality-oflife and survival data in health technology assessment.

A review by Billingham LJ, Abrams KR, Jones DR.

No. 11

Antenatal and neonatal

haemoglobinopathy screening in the

UK: review and economic analysis.

By Zeuner D, Ades AE, Karnon J,

Brown J, Dezateux C, Anionwu EN.

\section{No. 12}

Assessing the quality of reports of randomised trials: implications for the conduct of meta-analyses.

A review by Moher D, Cook DJ, Jadad AR, Tugwell P, Moher M, Jones A, et al.

No. 13

'Early warning systems' for identifying new healthcare technologies.

By Robert G, Stevens A, Gabbay J.

No. 14

A systematic review of the role of human papillomavirus testing within a cervical screening programme.

By Cuzick J, Sasieni P, Davies P, Adams J, Normand C, Frater A, et al.

\section{No. 15}

Near patient testing in diabetes clinics: appraising the costs and outcomes.

By Grieve R, Beech R, Vincent J, Mazurkiewicz J

\section{No. 16}

Positron emission tomography: establishing priorities for health technology assessment.

A review by Robert G, Milne R.
No. 17 (Pt 1)

The debridement of chronic wounds: a systematic review.

By Bradley M, Cullum N, Sheldon T.

\section{No. 17 (Pt 2)}

Systematic reviews of wound care management: (2) Dressings and topical agents used in the healing of chronic wounds.

By Bradley M, Cullum N, Nelson EA, Petticrew M, Sheldon T, Torgerson D.

\section{No. 18}

A systematic literature review of spiral and electron beam computed

tomography: with particular reference to clinical applications in hepatic lesions, pulmonary embolus and coronary artery disease.

By Berry E, Kelly S, Hutton J, Harris KM, Roderick P, Boyce JC, et al.

\section{No. 19}

What role for statins? A review and economic model.

By Ebrahim S, Davey Smith G McCabe C, Payne N, Pickin M, Sheldon TA, et al.

\section{No. 20}

Factors that limit the quality, number and progress of randomised controlled trials.

A review by Prescott RJ, Counsell CE, Gillespie WJ, Grant AM, Russell IT, Kiauka S, et al.

No. 21

Antimicrobial prophylaxis in total hip replacement: a systematic review.

By Glenny AM, Song F.

No. 22

Health promoting schools and health promotion in schools: two systematic reviews.

By Lister-Sharp D, Chapman S, Stewart-Brown S, Sowden A.

No. 23

Economic evaluation of a primary carebased education programme for patients with osteoarthritis of the knee.

A review by Lord I, Victor $C$, Littlejohns P, Ross FM, Axford JS.

\section{Volume 4, 2000}

No. 1

The estimation of marginal time preference in a UK-wide sample (TEMPUS) project.

A review by Cairns JA, van der Pol MM.

No. 2

Geriatric rehabilitation following fractures in older people: a systematic review.

By Cameron I, Crotty M, Currie C, Finnegan T, Gillespie L, Gillespie W, et al. 
No. 3

Screening for sickle cell disease and thalassaemia: a systematic review with supplementary research.

By Davies SC, Cronin E, Gill M, Greengross P, Hickman M, Normand C.

No. 4

Community provision of hearing aids and related audiology services.

A review by Reeves DJ, Alborz A, Hickson FS, Bamford JM.

No. 5

False-negative results in screening programmes: systematic review of impact and implications.

By Petticrew MP, Sowden AJ, Lister-Sharp D, Wright K.

No. 6

Costs and benefits of community postnatal support workers: a randomised controlled trial.

By Morrell CJ, Spiby H, Stewart P, Walters S, Morgan A.

No. 7

Implantable contraceptives (subdermal implants and hormonally impregnated intrauterine systems) versus other forms of reversible contraceptives: two systematic reviews to assess relative effectiveness, acceptability, tolerability and cost-effectiveness.

By French RS, Cowan FM, Mansour DJA, Morris S, Procter T, Hughes D, et al.

\section{No. 8}

An introduction to statistical methods for health technology assessment

A review by White SJ, Ashby D, Brown PJ.

No. 9

Disease-modifying drugs for multiple sclerosis: a rapid and systematic review.

By Clegg A, Bryant J, Milne R.

\section{No. 10}

Publication and related biases.

A review by Song F, Eastwood AJ, Gilbody S, Duley L, Sutton AJ.

No. 11

Cost and outcome implications of the organisation of vascular services.

By Michaels J, Brazier J, Palfreyman S, Shackley P, Slack R.

No. 12

Monitoring blood glucose control in diabetes mellitus: a systematic review.

By Coster S, Gulliford MC, Seed PT, Powrie JK, Swaminathan R.

No. 13

The effectiveness of domiciliary health visiting: a systematic review of international studies

and a selective review of the British literature.

By Elkan R, Kendrick D, Hewitt M, Robinson JJA, Tolley K, Blair M, et al.

\section{No. 14}

The determinants of screening uptake and interventions for increasing uptake: a systematic review.

By Jepson R, Clegg A, Forbes C, Lewis R, Sowden A, Kleijnen J.

\section{No. 15}

The effectiveness and cost-effectiveness of prophylactic removal of wisdom teeth.

A rapid review by Song F, O'Meara S, Wilson P, Golder S, Kleijnen J.

\section{No. 16}

Ultrasound screening in pregnancy: a systematic review of the clinical effectiveness, cost-effectiveness and women's views.

By Bricker L, Garcia J, Henderson J, Mugford M, Neilson J, Roberts T, et al.

\section{No. 17}

A rapid and systematic review of the effectiveness and cost-effectiveness of the taxanes used in the treatment of advanced breast and ovarian cancer.

By Lister-Sharp D, McDonagh MS, Khan KS, Kleijnen J.

\section{No. 18}

Liquid-based cytology in cervical screening: a rapid and systematic review.

By Payne N, Chilcott J, McGoogan E.

No. 19

Randomised controlled trial of nondirective counselling, cognitive-behaviour therapy and usual general practitioner care in the management of depression as well as mixed anxiety and depression in primary care.

By King M, Sibbald B, Ward E, Bower P, Lloyd M, Gabbay M, et al.

\section{No. 20}

Routine referral for radiography of patients presenting with low back pain: is patients' outcome influenced by GPs' referral for plain radiography?

By Kerry S, Hilton S, Patel S, Dundas D, Rink E, Lord J.

\section{No. 21}

Systematic reviews of wound care management: (3) antimicrobial agents for chronic wounds; (4) diabetic foot ulceration.

By O'Meara S, Cullum N, Majid M, Sheldon T.

\section{No. 22}

Using routine data to complement and enhance the results of randomised controlled trials.

By Lewsey JD, Leyland AH,

Murray GD, Boddy FA.

No. 23

Coronary artery stents in the treatment of ischaemic heart disease: a rapid and systematic review.

By Meads C, Cummins C, Jolly K, Stevens A, Burls A, Hyde C.
No. 24

Outcome measures for adult critical

care: a systematic review.

By Hayes JA, Black NA,

Jenkinson C, Young JD, Rowan KM, Daly K, et al.

\section{No. 25}

A systematic review to evaluate the effectiveness of interventions to promote the initiation of breastfeeding.

By Fairbank L, O'Meara S, Renfrew MJ, Woolridge M, Sowden AJ, Lister-Sharp D.

No. 26

Implantable cardioverter defibrillators: arrhythmias. A rapid and systematic review.

By Parkes J, Bryant J, Milne R.

No. 27

Treatments for fatigue in multiple sclerosis: a rapid and systematic review.

By Brañas P, Jordan R, Fry-Smith A, Burls A, Hyde C.

No. 28

Early asthma prophylaxis, natural history, skeletal development and economy (EASE): a pilot randomised controlled trial.

By Baxter-Jones ADG, Helms PJ, Russell G, Grant A, Ross S, Cairns JA, et al.

No. 29

Screening for hypercholesterolaemia versus case finding for familial hypercholesterolaemia: a systematic review and cost-effectiveness analysis.

By Marks D, Wonderling D,

Thorogood M, Lambert H, Humphries SE, Neil HAW.

No. 30

A rapid and systematic review of the clinical effectiveness and cost-

effectiveness of glycoprotein IIb/IIIa antagonists in the medical management of unstable angina.

By McDonagh MS, Bachmann LM, Golder S, Kleijnen J, ter Riet G.

No. 31

A randomised controlled trial of prehospital intravenous fluid replacement therapy in serious trauma.

By Turner J, Nicholl J, Webber L, Cox H, Dixon S, Yates D.

No. 32

Intrathecal pumps for giving opioids in chronic pain: a systematic review.

By Williams JE, Louw G, Towlerton G.

No. 33

Combination therapy (interferon alfa and ribavirin) in the treatment of chronic hepatitis C: a rapid and systematic review.

By Shepherd J, Waugh N, Hewitson P. 
No. 34

A systematic review of comparisons of effect sizes derived from randomised and non-randomised studies.

By MacLehose RR, Reeves BC, Harvey IM, Sheldon TA, Russell IT, Black AMS.

No. 35

Intravascular ultrasound-guided interventions in coronary artery disease: a systematic literature review, with decision-analytic modelling, of outcomes and cost-effectiveness.

By Berry E, Kelly S, Hutton J, Lindsay HSJ, Blaxill JM, Evans JA, et al.

No. 36

A randomised controlled trial to evaluate the effectiveness and costeffectiveness of counselling patients with chronic depression.

By Simpson S, Corney R, Fitzgerald P, Beecham J.

No. 37

Systematic review of treatments for atopic eczema.

By Hoare C, Li Wan Po A, Williams H.

No. 38

Bayesian methods in health technology assessment: a review.

By Spiegelhalter DJ, Myles JP, Jones DR, Abrams KR.

No. 39

The management of dyspepsia: a systematic review.

By Delaney B, Moayyedi P, Deeks J, Innes M, Soo S, Barton $\mathrm{P}$, et al.

No. 40

A systematic review of treatments for severe psoriasis.

By Griffiths CEM, Clark CM, Chalmers RJG, Li Wan Po A, Williams HC.

\section{Volume 5, 200I}

No. 1

Clinical and cost-effectiveness of donepezil, rivastigmine and galantamine for Alzheimer's disease: a rapid and systematic review.

By Clegg A, Bryant J, Nicholson T, McIntyre L, De Broe S, Gerard K, et al.

No. 2

The clinical effectiveness and costeffectiveness of riluzole for motor neurone disease: a rapid and systematic review.

By Stewart A, Sandercock J, Bryan S, Hyde C, Barton PM, Fry-Smith A, et al.

No. 3

Equity and the economic evaluation of healthcare.

By Sassi F, Archard L, Le Grand J.

No. 4

Quality-of-life measures in chronic

diseases of childhood.

By Eiser C, Morse R.
No. 5

Eliciting public preferences for

healthcare: a systematic review of techniques.

By Ryan M, Scott DA, Reeves C, Bate A, van Teijlingen ER, Russell EM, et al.

No. 6

General health status measures for people with cognitive impairment: learning disability and acquired brain injury.

By Riemsma RP, Forbes CA, Glanville JM, Eastwood AJ, Kleijnen J.

No. 7

An assessment of screening strategies for fragile $\mathrm{X}$ syndrome in the UK.

By Pembrey ME, Barnicoat AJ, Carmichael B, Bobrow M, Turner G.

No. 8

Issues in methodological research: perspectives from researchers and commissioners.

By Lilford RJ, Richardson A, Stevens A, Fitzpatrick R, Edwards S, Rock F, et al.

No. 9

Systematic reviews of wound care management: (5) beds; (6) compression;

(7) laser therapy, therapeutic

ultrasound, electrotherapy and

electromagnetic therapy.

By Cullum N, Nelson EA, Flemming

K, Sheldon T.

No. 10

Effects of educational and psychosocial interventions for adolescents with diabetes mellitus: a systematic review.

By Hampson SE, Skinner TC, Hart J, Storey L, Gage H, Foxcroft D, et al.

\section{No. 11}

Effectiveness of autologous chondrocyte transplantation for hyaline cartilage defects in knees: a rapid and systematic review.

By Jobanputra P, Parry D, Fry-Smith A, Burls A.

\section{No. 12}

Statistical assessment of the learning curves of health technologies.

By Ramsay CR, Grant AM,

Wallace SA, Garthwaite PH, Monk AF, Russell IT.

\section{No. 13}

The effectiveness and cost-effectiveness of temozolomide for the treatment of recurrent malignant glioma: a rapid and systematic review.

By Dinnes J, Cave C, Huang S, Major K, Milne R.

\section{No. 14}

A rapid and systematic review of the clinical effectiveness and costeffectiveness of debriding agents in treating surgical wounds healing by secondary intention.

By Lewis R, Whiting P, ter Riet G, O’Meara S, Glanville J.
No. 15

Home treatment for mental health

problems: a systematic review.

By Burns T, Knapp M,

Catty J, Healey A, Henderson J, Watt $\mathrm{H}$, et al.

\section{No. 16}

How to develop cost-conscious guidelines.

By Eccles M, Mason J.

No. 17

The role of specialist nurses in multiple sclerosis: a rapid and systematic review.

By De Broe S, Christopher F, Waugh N.

No. 18

A rapid and systematic review of the clinical effectiveness and costeffectiveness of orlistat in the management of obesity.

By O'Meara S, Riemsma R, Shirran L, Mather L, ter Riet G.

\section{No. 19}

The clinical effectiveness and costeffectiveness of pioglitazone for type 2 diabetes mellitus: a rapid and systematic review.

By Chilcott J, Wight J, Lloyd Jones M, Tappenden P.

\section{No. 20}

Extended scope of nursing practice: a multicentre randomised controlled trial of appropriately trained nurses and preregistration house officers in preoperative assessment in elective general surgery.

By Kinley H, Czoski-Murray C, George S, McCabe C, Primrose J, Reilly C, et al.

No. 21

Systematic reviews of the effectiveness of day care for people with severe mental disorders: (1) Acute day hospital versus admission; (2) Vocational rehabilitation; (3) Day hospital versus outpatient care.

By Marshall M, Crowther R, AlmarazSerrano A, Creed F, Sledge W, Kluiter $\mathrm{H}$, et al.

No. 22

The measurement and monitoring of surgical adverse events.

By Bruce J, Russell EM, Mollison J, Krukowski ZH.

\section{No. 23}

Action research: a systematic review and guidance for assessment.

By Waterman H, Tillen D, Dickson R, de Koning $\mathbf{K}$.

No. 24

A rapid and systematic review of the clinical effectiveness and costeffectiveness of gemcitabine for the treatment of pancreatic cancer.

By Ward S, Morris E, Bansback N, Calvert N, Crellin A, Forman D, et al. 


\section{No. 25}

A rapid and systematic review of the evidence for the clinical effectiveness and cost-effectiveness of irinotecan, oxaliplatin and raltitrexed for the treatment of advanced colorectal cancer.

By Lloyd Jones M, Hummel S, Bansback N, Orr B, Seymour M.

\section{No. 26}

Comparison of the effectiveness of inhaler devices in asthma and chronic obstructive airways disease: a systematic review of the literature.

By Brocklebank D, Ram F, Wright J, Barry P, Cates C, Davies L, et al.

\section{No. 27}

The cost-effectiveness of magnetic resonance imaging for investigation of the knee joint.

By Bryan S, Weatherburn G, Bungay

H, Hatrick C, Salas C, Parry D, et al.

\section{No. 28}

A rapid and systematic review of the clinical effectiveness and costeffectiveness of topotecan for ovarian cancer.

By Forbes C, Shirran L, Bagnall A-M, Duffy S, ter Riet G.

\section{No. 29}

Superseded by a report published in a later volume.

\section{No. 30}

The role of radiography in primary care patients with low back pain of at least 6 weeks duration: a randomised (unblinded) controlled trial.

By Kendrick D, Fielding K, Bentley E, Miller P, Kerslake R, Pringle M.

\section{No. 31}

Design and use of questionnaires: a review of best practice applicable to surveys of health service staff and patients.

By McColl E, Jacoby A, Thomas L, Soutter J, Bamford C, Steen N, et al.

\section{No. 32}

A rapid and systematic review of the clinical effectiveness and cost-

effectiveness of paclitaxel, docetaxel, gemcitabine and vinorelbine in nonsmall-cell lung cancer.

By Clegg A, Scott DA, Sidhu M, Hewitson P, Waugh N.

\section{No. 33}

Subgroup analyses in randomised controlled trials: quantifying the risks of false-positives and false-negatives.

By Brookes ST, Whitley E, Peters TJ, Mulheran PA, Egger M, Davey Smith G.

\section{No. 34}

Depot antipsychotic medication in the treatment of patients with schizophrenia: (1) Meta-review; (2) Patient and nurse attitudes.

By David AS, Adams C.

\section{No. 35}

A systematic review of controlled trials of the effectiveness and costeffectiveness of brief psychological treatments for depression.

By Churchill R, Hunot V, Corney R, Knapp M, McGuire H, Tylee A, et al.

No. 36

Cost analysis of child health surveillance.

By Sanderson D, Wright D, Acton C, Duree D.

\section{Volume 6, 2002}

\section{No. 1}

A study of the methods used to select review criteria for clinical audit.

By Hearnshaw H, Harker R, Cheater F, Baker R, Grimshaw G.

No. 2

Fludarabine as second-line therapy for B cell chronic lymphocytic leukaemia: a technology assessment.

By Hyde C, Wake B, Bryan S, Barton P, Fry-Smith A, Davenport C, et al.

No. 3

Rituximab as third-line treatment for refractory or recurrent Stage III or IV follicular non-Hodgkin's lymphoma: a systematic review and economic evaluation.

By Wake B, Hyde C, Bryan S, Barton P, Song F, Fry-Smith A, et al.

No. 4

A systematic review of discharge arrangements for older people.

By Parker SG, Peet SM, McPherson A, Cannaby AM, Baker R, Wilson A, et al.

No. 5

The clinical effectiveness and costeffectiveness of inhaler devices used in the routine management of chronic asthma in older children: a systematic review and economic evaluation.

By Peters J, Stevenson M, Beverley C, Lim J, Smith S.

No. 6

The clinical effectiveness and costeffectiveness of sibutramine in the management of obesity: a technology assessment.

By O'Meara S, Riemsma R, Shirran L, Mather L, ter Riet G.

No. 7

The cost-effectiveness of magnetic resonance angiography for carotid artery stenosis and peripheral vascular disease: a systematic review.

By Berry E, Kelly S, Westwood ME, Davies LM, Gough MJ, Bamford JM, et al.

No. 8

Promoting physical activity in South Asian Muslim women through 'exercise on prescription'.

By Carroll B, Ali N, Azam N.
No. 9

Zanamivir for the treatment of influenza

in adults: a systematic review and economic evaluation.

By Burls A, Clark W, Stewart T, Preston C, Bryan S, Jefferson T, et al.

\section{No. 10}

A review of the natural history and epidemiology of multiple sclerosis: implications for resource allocation and health economic models.

By Richards RG, Sampson FC, Beard SM, Tappenden P.

No. 11

Screening for gestational diabetes: a systematic review and economic evaluation.

By Scott DA, Loveman E, McIntyre L, Waugh N.

No. 12

The clinical effectiveness and costeffectiveness of surgery for people with morbid obesity: a systematic review and economic evaluation.

By Clegg AJ, Colquitt J, Sidhu MK, Royle P, Loveman E, Walker A.

\section{No. 13}

The clinical effectiveness of trastuzumab for breast cancer: a systematic review.

By Lewis R, Bagnall A-M, Forbes C, Shirran E, Duffy S, Kleijnen J, et al.

\section{No. 14}

The clinical effectiveness and costeffectiveness of vinorelbine for breast cancer: a systematic review and economic evaluation.

By Lewis R, Bagnall A-M, King S, Woolacott N, Forbes C, Shirran L, et al.

\section{No. 15}

A systematic review of the effectiveness and cost-effectiveness of metal-on-metal hip resurfacing arthroplasty for treatment of hip disease.

By Vale L, Wyness L, McCormack K, McKenzie L, Brazzelli M, Stearns SC.

\section{No. 16}

The clinical effectiveness and costeffectiveness of bupropion and nicotine replacement therapy for smoking cessation: a systematic review and economic evaluation.

By Woolacott NF, Jones L, Forbes CA, Mather LC, Sowden AJ, Song FJ, et al.

No. 17

A systematic review of effectiveness and economic evaluation of new drug treatments for juvenile idiopathic arthritis: etanercept.

By Cummins C, Connock M, Fry-Smith A, Burls A.

No. 18

Clinical effectiveness and costeffectiveness of growth hormone in children: a systematic review and economic evaluation.

By Bryant J, Cave C, Mihaylova B, Chase D, McIntyre L, Gerard K, et al. 
No. 19

Clinical effectiveness and cost-

effectiveness of growth hormone in adults in relation to impact on quality of life: a systematic review and economic evaluation.

By Bryant J, Loveman E, Chase D, Mihaylova B, Cave C, Gerard K, et al.

\section{No. 20}

Clinical medication review by a pharmacist of patients on repeat prescriptions in general practice: a randomised controlled trial.

By Zermansky AG, Petty DR, Raynor DK, Lowe CJ, Freementle N, Vail A.

No. 21

The effectiveness of infliximab and etanercept for the treatment of rheumatoid arthritis: a systematic review and economic evaluation.

By Jobanputra P, Barton P, Bryan S, Burls A.

No. 22

A systematic review and economic evaluation of computerised cognitive behaviour therapy for depression and anxiety.

By Kaltenthaler E, Shackley P, Stevens K, Beverley C, Parry G, Chilcott J.

No. 23

A systematic review and economic evaluation of pegylated liposomal doxorubicin hydrochloride for ovarian cancer.

By Forbes C, Wilby J, Richardson G, Sculpher M, Mather L, Reimsma R.

No. 24

A systematic review of the effectiveness of interventions based on a stages-ofchange approach to promote individual behaviour change.

By Riemsma RP, Pattenden J, Bridle C, Sowden AJ, Mather L, Watt IS, et al.

No. 25

A systematic review update of the clinical effectiveness and costeffectiveness of glycoprotein IIb/IIIa antagonists.

By Robinson M, Ginnelly L, Sculpher M, Jones L, Riemsma R, Palmer S, et al.

No. 26

A systematic review of the effectiveness, cost-effectiveness and barriers to implementation of thrombolytic and neuroprotective therapy for acute ischaemic stroke in the NHS.

By Sandercock P, Berge E, Dennis M, Forbes J, Hand P, Kwan J, et al.

No. 27

A randomised controlled crossover trial of nurse practitioner versus doctor-led outpatient care in a bronchiectasis clinic.

By Caine N, Sharples LD,

Hollingworth W, French J, Keogan M, Exley A, et al.
No. 28

Clinical effectiveness and cost consequences of selective serotonin reuptake inhibitors in the treatment of sex offenders.

By Adi Y, Ashcroft D, Browne K, Beech A, Fry-Smith A, Hyde C.

\section{No. 29}

Treatment of established osteoporosis: a systematic review and cost-utility analysis.

By Kanis JA, Brazier JE, Stevenson M, Calvert NW, Lloyd Jones M.

No. 30

Which anaesthetic agents are costeffective in day surgery? Literature review, national survey of practice and randomised controlled trial.

By Elliott RA Payne K, Moore JK,

Davies LM, Harper NJN, St Leger AS, et al.

\section{No. 31}

Screening for hepatitis $\mathrm{C}$ among injecting drug users and in genitourinary medicine clinics: systematic reviews of effectiveness, modelling study and national survey of current practice.

By Stein K, Dalziel K, Walker A, McIntyre L, Jenkins B, Horne J, et al.

No. 32

The measurement of satisfaction with healthcare: implications for practice from a systematic review of the literature.

By Crow R, Gage H, Hampson S, Hart J, Kimber A, Storey L, et al.

No. 33

The effectiveness and cost-effectiveness of imatinib in chronic myeloid leukaemia: a systematic review.

By Garside R, Round A, Dalziel K, Stein K, Royle R.

\section{No. 34}

A comparative study of hypertonic saline, daily and alternate-day rhDNase in children with cystic fibrosis.

By Suri R, Wallis C, Bush A,

Thompson S, Normand C, Flather M et al.

No. 35

A systematic review of the costs and effectiveness of different models of paediatric home care.

By Parker G, Bhakta P, Lovett CA, Paisley S, Olsen R, Turner D, et al.

\section{Volume 7, 2003}

No. 1

How important are comprehensive literature searches and the assessment of trial quality in systematic reviews? Empirical study.

By Egger M, Jüni P, Bartlett C, Holenstein F, Sterne J.

\section{No. 2}

Systematic review of the effectiveness and cost-effectiveness, and economic evaluation, of home versus hospital or satellite unit haemodialysis for people with end-stage renal failure.

By Mowatt G, Vale L, Perez J, Wyness L, Fraser C, MacLeod A, et al.

No. 3

Systematic review and economic evaluation of the effectiveness of infliximab for the treatment of Crohn's disease.

By Clark W, Raftery J, Barton P, Song F, Fry-Smith A, Burls A.

No. 4

A review of the clinical effectiveness and cost-effectiveness of routine anti-D prophylaxis for pregnant women who are rhesus negative.

By Chilcott J, Lloyd Jones M, Wight J, Forman K, Wray J, Beverley C, et al.

No. 5

Systematic review and evaluation of the use of tumour markers in paediatric oncology: Ewing's sarcoma and neuroblastoma.

By Riley RD, Burchill SA, Abrams KR, Heney D, Lambert PC, Jones DR, et al.

No. 6

The cost-effectiveness of screening for Helicobacter pylori to reduce mortality and morbidity from gastric cancer and peptic ulcer disease: a discrete-event simulation model.

By Roderick P, Davies R, Raftery J, Crabbe D, Pearce R, Bhandari P, et al.

No. 7

The clinical effectiveness and costeffectiveness of routine dental checks: a systematic review and economic evaluation.

By Davenport C, Elley K, Salas C, Taylor-Weetman CL, Fry-Smith A, Bryan S, et al.

\section{No. 8}

A multicentre randomised controlled trial assessing the costs and benefits of using structured information and analysis of women's preferences in the management of menorrhagia.

By Kennedy ADM, Sculpher MJ, Coulter A, Dwyer N, Rees M, Horsley S, et al.

No. 9

Clinical effectiveness and cost-utility of photodynamic therapy for wet age-related macular degeneration: a systematic review and economic evaluation.

By Meads C, Salas C, Roberts T, Moore D, Fry-Smith A, Hyde C.

\section{No. 10}

Evaluation of molecular tests for prenatal diagnosis of chromosome abnormalities.

By Grimshaw GM, Szczepura A,

Hultén M, MacDonald F, Nevin NC, Sutton F, et al. 
No. 11

First and second trimester antenatal screening for Down's syndrome: the results of the Serum, Urine and Ultrasound Screening Study (SURUSS).

By Wald NJ, Rodeck C, Hackshaw AK, Walters J, Chitty L, Mackinson AM.

No. 12

The effectiveness and cost-effectiveness of ultrasound locating devices for central venous access: a systematic review and economic evaluation.

By Calvert N, Hind D, McWilliams RG, Thomas SM, Beverley C, Davidson A

No. 13

A systematic review of atypical antipsychotics in schizophrenia.

By Bagnall A-M, Jones L, Lewis R, Ginnelly L, Glanville J, Torgerson D, et al.

No. 14

Prostate Testing for Cancer and Treatment (ProtecT) feasibility study.

By Donovan J, Hamdy F, Neal D, Peters T, Oliver S, Brindle L, et al.

\section{No. 15}

Early thrombolysis for the treatment of acute myocardial infarction: a systematic review and economic evaluation.

By Boland A, Dundar Y, Bagust A, Haycox A, Hill R, Mujica Mota R, et al.

\section{No. 16}

Screening for fragile $\mathrm{X}$ syndrome: a literature review and modelling.

By Song FJ, Barton P, Sleightholme V, Yao GL, Fry-Smith A

\section{No. 17}

Systematic review of endoscopic sinus surgery for nasal polyps.

By Dalziel K, Stein K, Round A, Garside R, Royle P.

\section{No. 18}

Towards efficient guidelines: how to monitor guideline use in primary care.

By Hutchinson A, McIntosh A, Cox S, Gilbert C.

\section{No. 19}

Effectiveness and cost-effectiveness of acute hospital-based spinal cord injuries services: systematic review.

By Bagnall A-M, Jones L,

Richardson G, Duffy S,

Riemsma R.

\section{No. 20}

Prioritisation of health technology assessment. The PATHS model: methods and case studies.

By Townsend J, Buxton M, Harper G.
No. 21

Systematic review of the clinical effectiveness and cost-effectiveness of tension-free vaginal tape for treatment of urinary stress incontinence.

By Cody J, Wyness L, Wallace S, Glazener C, Kilonzo M, Stearns S, et al.

\section{No. 22}

The clinical and cost-effectiveness of patient education models for diabetes: a systematic review and economic evaluation.

By Loveman E, Cave C, Green C, Royle P, Dunn N, Waugh N.

\section{No. 23}

The role of modelling in prioritising and planning clinical trials.

By Chilcott J, Brennan A, Booth A, Karnon J, Tappenden P.

No. 24

Cost-benefit evaluation of routine influenza immunisation in people $65-74$ years of age.

By Allsup S, Gosney M, Haycox A, Regan M.

No. 25

The clinical and cost-effectiveness of pulsatile machine perfusion versus cold storage of kidneys for transplantation retrieved from heart-beating and nonheart-beating donors.

By Wight J, Chilcott J, Holmes M, Brewer N

\section{No. 26}

Can randomised trials rely on existing electronic data? A feasibility study to explore the value of routine data in health technology assessment.

By Williams JG, Cheung WY,

Cohen DR, Hutchings HA, Longo MF, Russell IT.

No. 27

Evaluating non-randomised intervention studies.

By Deeks JJ, Dinnes J, D’Amico R, Sowden AJ, Sakarovitch C, Song F, et al.

No. 28

A randomised controlled trial to assess the impact of a package comprising a patient-orientated, evidence-based selfhelp guidebook and patient-centred consultations on disease management and satisfaction in inflammatory bowel disease.

By Kennedy A, Nelson E, Reeves D, Richardson G, Roberts C, Robinson A, et al.

\section{No. 29}

The effectiveness of diagnostic tests for the assessment of shoulder pain due to soft tissue disorders: a systematic review.

By Dinnes J, Loveman E, McIntyre L, Waugh $\mathrm{N}$.
No. 30

The value of digital imaging in diabetic retinopathy.

By Sharp PF, Olson J, Strachan F, Hipwell J, Ludbrook A, O'Donnell M, et al.

No. 31

Lowering blood pressure to prevent myocardial infarction and stroke: a new preventive strategy.

By Law M, Wald N, Morris J.

No. 32

Clinical and cost-effectiveness of capecitabine and tegafur with uracil for the treatment of metastatic colorectal cancer: systematic review and economic evaluation.

By Ward S, Kaltenthaler E, Cowan J, Brewer N.

No. 33

Clinical and cost-effectiveness of new and emerging technologies for early localised prostate cancer: a systematic review.

By Hummel S, Paisley S, Morgan A, Currie E, Brewer N.

No. 34

Literature searching for clinical and cost-effectiveness studies used in health technology assessment reports carried out for the National Institute for Clinical Excellence appraisal system

By Royle P, Waugh N.

No. 35

Systematic review and economic decision modelling for the prevention and treatment of influenza

$\mathrm{A}$ and $\mathrm{B}$.

By Turner D, Wailoo A, Nicholson K, Cooper N, Sutton A, Abrams K.

No. 36

A randomised controlled trial to evaluate the clinical and costeffectiveness of Hickman line insertions in adult cancer patients by nurses.

By Boland A, Haycox A, Bagust A, Fitzsimmons L.

No. 37

Redesigning postnatal care: a randomised controlled trial of protocol-based midwifery-led care focused on individual women's physical and psychological health needs.

By MacArthur C, Winter HR,

Bick DE, Lilford RJ, Lancashire RJ, Knowles $\mathrm{H}$, et al.

No. 38

Estimating implied rates of discount in healthcare decision-making.

By West RR, McNabb R,

Thompson AGH, Sheldon TA, Grimley Evans J. 
No. 39

Systematic review of isolation policies in the hospital management of methicillinresistant Staphylococcus aureus: a review of the literature with epidemiological and economic modelling.

By Cooper BS, Stone SP, Kibbler CC, Cookson BD, Roberts JA, Medley GF, et al.

No. 40

Treatments for spasticity and pain in multiple sclerosis: a systematic review.

By Beard S, Hunn A, Wight J.

No. 41

The inclusion of reports of randomised trials published in languages other than English in systematic reviews.

By Moher D, Pham B, Lawson ML, Klassen TP.

No. 42

The impact of screening on future health-promoting behaviours and health beliefs: a systematic review.

By Bankhead CR, Brett J, Bukach C, Webster P, Stewart-Brown S, Munafo M, et al.

\section{Volume 8, 2004}

No. 1

What is the best imaging strategy for acute stroke?

By Wardlaw JM, Keir SL, Seymour J, Lewis S, Sandercock PAG, Dennis MS, et al.

No. 2

Systematic review and modelling of the investigation of acute and chronic chest pain presenting in primary care.

By Mant J, McManus RJ, Oakes RAL, Delaney BC, Barton PM, Deeks JJ, et al.

No. 3

The effectiveness and cost-effectiveness of microwave and thermal balloon endometrial ablation for heavy menstrual bleeding: a systematic review and economic modelling.

By Garside R, Stein K, Wyatt K, Round A, Price A.

No. 4

A systematic review of the role of bisphosphonates in metastatic disease.

By Ross JR, Saunders Y, Edmonds PM, Patel S, Wonderling D, Normand C, et al.

No. 5

Systematic review of the clinical effectiveness and cost-effectiveness of capecitabine (Xeloda ${ }^{\circledR}$ ) for locally advanced and/or metastatic breast cancer.

By Jones L, Hawkins N, Westwood M, Wright K, Richardson G, Riemsma R.

No. 6

Effectiveness and efficiency of guideline dissemination and implementation strategies.

By Grimshaw JM, Thomas RE, MacLennan G, Fraser C, Ramsay CR,
No. 7

Clinical effectiveness and costs of the Sugarbaker procedure for the treatment of pseudomyxoma peritonei.

By Bryant J, Clegg AJ, Sidhu MK, Brodin H, Royle P, Davidson P.

No. 8

Psychological treatment for insomnia in the regulation of long-term hypnotic drug use.

By Morgan K, Dixon S, Mathers N, Thompson J, Tomeny M.

No. 9

Improving the evaluation of therapeutic interventions in multiple sclerosis: development of a patient-based measure of outcome.

By Hobart JC, Riazi A, Lamping DL, Fitzpatrick R, Thompson AJ.

\section{No. 10}

A systematic review and economic evaluation of magnetic resonance cholangiopancreatography compared with diagnostic endoscopic retrograde cholangiopancreatography.

By Kaltenthaler E, Bravo Vergel Y, Chilcott J, Thomas S, Blakeborough T, Walters SJ, et al.

\section{No. 11}

The use of modelling to evaluate new drugs for patients with a chronic condition: the case of antibodies against tumour necrosis factor in rheumatoid arthritis.

By Barton P, Jobanputra P, Wilson J, Bryan S, Burls A.

No. 12

Clinical effectiveness and costeffectiveness of neonatal screening for inborn errors of metabolism using tandem mass spectrometry: a systematic review.

By Pandor A, Eastham J, Beverley C, Chilcott J, Paisley S.

\section{No. 13}

Clinical effectiveness and costeffectiveness of pioglitazone and rosiglitazone in the treatment of type 2 diabetes: a systematic review and economic evaluation.

By Czoski-Murray C, Warren E, Chilcott J, Beverley C, Psyllaki MA, Cowan J.

\section{No. 14}

Routine examination of the newborn: the EMREN study. Evaluation of an extension of the midwife role including a randomised controlled trial of appropriately trained midwives and paediatric senior house officers.

By Townsend J, Wolke D, Hayes J, Davé S, Rogers C, Bloomfield L, et al.
No. 15

Involving consumers in research and development agenda setting for the NHS: developing an evidence-based approach.

By Oliver S, Clarke-Jones L, Rees R, Milne R, Buchanan P, Gabbay J, et al.

No. 16

A multi-centre randomised controlled trial of minimally invasive direct coronary bypass grafting versus percutaneous transluminal coronary angioplasty with stenting for proximal stenosis of the left anterior descending coronary artery.

By Reeves BC, Angelini GD, Bryan AJ, Taylor FC, Cripps T, Spyt TJ, et al.

No. 17

Does early magnetic resonance imaging influence management or improve outcome in patients referred to secondary care with low back pain? A pragmatic randomised controlled trial.

By Gilbert FJ, Grant AM, Gillan MGC, Vale L, Scott NW, Campbell MK, et al.

No. 18

The clinical and cost-effectiveness of anakinra for the treatment of rheumatoid arthritis in adults: a systematic review and economic analysis.

By Clark W, Jobanputra P, Barton P, Burls A.

No. 19

A rapid and systematic review and economic evaluation of the clinical and cost-effectiveness of newer drugs for treatment of mania associated with bipolar affective disorder

By Bridle C, Palmer S, Bagnall A-M, Darba J, Duffy S, Sculpher M, et al.

No. 20

Liquid-based cytology in cervical screening: an updated rapid and systematic review and economic analysis.

By Karnon J, Peters J, Platt J, Chilcott J, McGoogan E, Brewer N.

No. 21

Systematic review of the long-term effects and economic consequences of treatments for obesity and implications for health improvement.

By Avenell A, Broom J, Brown TJ, Poobalan A, Aucott L, Stearns SC, et al.

No. 22

Autoantibody testing in children with newly diagnosed type 1 diabetes mellitus.

By Dretzke J, Cummins C, Sandercock J, Fry-Smith A, Barrett T, Burls A. 
No. 23

Clinical effectiveness and cost-

effectiveness of prehospital intravenous fluids in trauma patients.

By Dretzke J, Sandercock J, Bayliss S, Burls A.

\section{No. 24}

Newer hypnotic drugs for the shortterm management of insomnia: a systematic review and economic evaluation.

By Dündar Y, Boland A, Strobl J, Dodd S, Haycox A, Bagust A, et al.

\section{No. 25}

Development and validation of methods for assessing the quality of diagnostic accuracy studies.

By Whiting P, Rutjes AWS, Dinnes J, Reitsma JB, Bossuyt PMM, Kleijnen J.

No. 26

EVALUATE hysterectomy trial: a multicentre randomised trial comparing abdominal, vaginal and laparoscopic methods of hysterectomy.

By Garry R, Fountain J, Brown J, Manca A, Mason S, Sculpher M, et al.

\section{No. 27}

Methods for expected value of information analysis in complex health economic models: developments on the health economics of interferon- $\beta$ and glatiramer acetate for multiple sclerosis.

By Tappenden P, Chilcott JB, Eggington S, Oakley J, McCabe C.

No. 28

Effectiveness and cost-effectiveness of imatinib for first-line treatment of chronic myeloid leukaemia in chronic phase: a systematic review and economic analysis

By Dalziel K, Round A, Stein K, Garside R, Price A.

\section{No. 29}

VenUS I: a randomised controlled trial of two types of bandage for treating venous leg ulcers.

By Iglesias C, Nelson EA, Cullum NA, Torgerson DJ on behalf of the VenUS Team.

\section{No. 30}

Systematic review of the effectiveness and cost-effectiveness, and economic evaluation, of myocardial perfusion scintigraphy for the diagnosis and management of angina and myocardial infarction.

By Mowatt G, Vale L, Brazzelli M, Hernandez R, Murray A, Scott N, et al.

\section{No. 31}

A pilot study on the use of decision theory and value of information analysis as part of the NHS Health Technology Assessment programme.

By Claxton K, Ginnelly L, Sculpher M, Philips Z, Palmer S.
No. 32

The Social Support and Family Health Study: a randomised controlled trial and economic evaluation of two alternative forms of postnatal support for mothers living in disadvantaged inner-city areas.

By Wiggins M, Oakley A, Roberts I,

Turner H, Rajan L, Austerberry H, et al.

No. 33

Psychosocial aspects of genetic screening of pregnant women and newborns: a systematic review.

By Green JM, Hewison J, Bekker HL, Bryant, Cuckle HS.

No. 34

Evaluation of abnormal uterine

bleeding: comparison of three outpatient procedures within cohorts defined by age and menopausal status.

By Critchley HOD, Warner P,

Lee AJ, Brechin S, Guise J, Graham B.

\section{No. 35}

Coronary artery stents: a rapid systematic review and economic evaluation.

By Hill R, Bagust A, Bakhai A, Dickson R, Dündar Y, Haycox A, et al.

No. 36

Review of guidelines for good practice in decision-analytic modelling in health technology assessment.

By Philips Z, Ginnelly L, Sculpher M, Claxton K, Golder S, Riemsma R, et al.

No. 37

Rituximab (MabThera ${ }^{\circledR}$ ) for aggressive non-Hodgkin's lymphoma: systematic review and economic evaluation.

By Knight C, Hind D, Brewer N, Abbott V.

No. 38

Clinical effectiveness and costeffectiveness of clopidogrel and modified-release dipyridamole in the secondary prevention of occlusive vascular events: a systematic review and economic evaluation.

By Jones L, Griffin S, Palmer S, Main C, Orton V, Sculpher M, et al.

\section{No. 39}

Pegylated interferon $\alpha-2 \mathrm{a}$ and -2b in combination with ribavirin in the treatment of chronic hepatitis C: a systematic review and economic evaluation.

By Shepherd J, Brodin H, Cave C, Waugh N, Price A, Gabbay J.

\section{No. 40}

Clopidogrel used in combination with aspirin compared with aspirin alone in the treatment of non-ST-segmentelevation acute coronary syndromes: a systematic review and economic evaluation.

By Main C, Palmer S, Griffin S, Jones L, Orton V, Sculpher M, et al.
No. 41

Provision, uptake and cost of cardiac rehabilitation programmes: improving services to under-represented groups.

By Beswick AD, Rees K, Griebsch I, Taylor FC, Burke M, West RR, et al.

\section{No. 42}

Involving South Asian patients in clinical trials.

By Hussain-Gambles M, Leese B, Atkin K, Brown J, Mason S, Tovey P.

No. 43

Clinical and cost-effectiveness of continuous subcutaneous insulin infusion for diabetes.

By Colquitt JL, Green C, Sidhu MK, Hartwell D, Waugh N.

No. 44

Identification and assessment of ongoing trials in health technology assessment reviews.

By Song FJ, Fry-Smith A, Davenport C, Bayliss S, Adi Y, Wilson JS, et al.

No. 45

Systematic review and economic evaluation of a long-acting insulin analogue, insulin glargine

By Warren E, Weatherley-Jones E, Chilcott J, Beverley C.

\section{No. 46}

Supplementation of a home-based exercise programme with a class-based programme for people with osteoarthritis of the knees: a randomised controlled trial and health economic analysis.

By McCarthy CJ, Mills PM,

Pullen R, Richardson G, Hawkins N, Roberts CR, et al.

\section{No. 47}

Clinical and cost-effectiveness of oncedaily versus more frequent use of same potency topical corticosteroids for atopic eczema: a systematic review and economic evaluation.

By Green C, Colquitt JL, Kirby J, Davidson P, Payne E.

No. 48

Acupuncture of chronic headache disorders in primary care: randomised controlled trial and economic analysis.

By Vickers AJ, Rees RW, Zollman CE, McCarney R, Smith CM, Ellis N, et al.

\section{No. 49}

Generalisability in economic evaluation studies in healthcare: a review and case studies.

By Sculpher MJ, Pang FS, Manca A Drummond MF, Golder S, Urdahl H, et al.

\section{No. 50}

Virtual outreach: a randomised controlled trial and economic evaluation of joint teleconferenced medical consultations.

By Wallace P, Barber J, Clayton W, Currell R, Fleming K, Garner P, et al. 


\section{Volume 9, 2005}

No. 1

Randomised controlled multiple treatment comparison to provide a cost-effectiveness rationale for the selection of antimicrobial therapy in acne.

By Ozolins M, Eady EA, Avery A, Cunliffe WJ, O’Neill C, Simpson NB, et al.

No. 2

Do the findings of case series studies vary significantly according to methodological characteristics?

By Dalziel K, Round A, Stein K, Garside R, Castelnuovo E, Payne L.

No. 3

Improving the referral process for familial breast cancer genetic counselling: findings of three randomised controlled trials of two interventions.

By Wilson BJ, Torrance N, Mollison J, Wordsworth S, Gray JR, Haites NE, et al.

No. 4

Randomised evaluation of alternative electrosurgical modalities to treat bladder outflow obstruction in men with benign prostatic hyperplasia.

By Fowler C, McAllister W, Plail R, Karim O, Yang $Q$.

No. 5

A pragmatic randomised controlled trial of the cost-effectiveness of palliative therapies for patients with inoperable oesophageal cancer.

By Shenfine J, McNamee P, Steen N, Bond J, Griffin SM.

No. 6

Impact of computer-aided detection prompts on the sensitivity and specificity of screening mammography.

By Taylor P, Champness J, GivenWilson R, Johnston K, Potts H.

No. 7

Issues in data monitoring and interim analysis of trials.

By Grant AM, Altman DG, Babiker $\mathrm{AB}$, Campbell MK, Clemens FJ, Darbyshire JH, et al.

No. 8

Lay public's understanding of equipoise and randomisation in randomised controlled trials.

By Robinson EJ, Kerr CEP, Stevens AJ, Lilford RJ, Braunholtz DA, Edwards SJ, et al.

No. 9

Clinical and cost-effectiveness of electroconvulsive therapy for depressive illness, schizophrenia, catatonia and mania: systematic reviews and economic modelling studies.

By Greenhalgh J, Knight C, Hind D, Beverley C, Walters S.

\section{No. 10}

Measurement of health-related quality of life for people with dementia: development of a new instrument (DEMQOL) and an evaluation of current methodology.

By Smith SC, Lamping DL,

Banerjee S, Harwood R, Foley B, Smith P, et al.

\section{No. 11}

Clinical effectiveness and costeffectiveness of drotrecogin alfa (activated) $\left(\right.$ Xigris $^{\circledR}$ ) for the treatment of severe sepsis in adults: a systematic review and economic evaluation.

By Green C, Dinnes J, Takeda A, Shepherd J, Hartwell D, Cave C, et al.

\section{No. 12}

A methodological review of how heterogeneity has been examined in systematic reviews of diagnostic test accuracy.

By Dinnes J, Deeks J, Kirby J, Roderick P.

\section{No. 13}

Cervical screening programmes: can automation help? Evidence from systematic reviews, an economic analysis and a simulation modelling exercise applied to the UK.

By Willis BH, Barton P, Pearmain P, Bryan S, Hyde C.

\section{No. 14}

Laparoscopic surgery for inguinal hernia repair: systematic review of effectiveness and economic evaluation.

By McCormack K, Wake B, Perez J, Fraser C, Cook J, McIntosh E, et al.

\section{No. 15}

Clinical effectiveness, tolerability and cost-effectiveness of newer drugs for epilepsy in adults: a systematic review and economic evaluation.

By Wilby J, Kainth A, Hawkins N, Epstein D, McIntosh H, McDaid C, et al.

\section{No. 16}

A randomised controlled trial to compare the cost-effectiveness of tricyclic antidepressants, selective serotonin reuptake inhibitors and lofepramine.

By Peveler R, Kendrick T, Buxton M, Longworth L, Baldwin D, Moore M, et al.

\section{No. 17}

Clinical effectiveness and costeffectiveness of immediate angioplasty for acute myocardial infarction: systematic review and economic evaluation.

By Hartwell D, Colquitt J, Loveman E, Clegg AJ, Brodin H, Waugh N, et al.
No. 18

A randomised controlled comparison of alternative strategies in stroke care.

By Kalra L, Evans A, Perez I,

Knapp M, Swift C, Donaldson N.

No. 19

The investigation and analysis of critical incidents and adverse events in healthcare.

By Woloshynowych M, Rogers S, Taylor-Adams S, Vincent C.

No. 20

Potential use of routine databases in health technology assessment.

By Raftery J, Roderick P, Stevens A.

No. 21

Clinical and cost-effectiveness of newer immunosuppressive regimens in renal transplantation: a systematic review and modelling study.

By Woodroffe R, Yao GL, Meads C, Bayliss S, Ready A, Raftery J, et al.

No. 22

A systematic review and economic evaluation of alendronate, etidronate, risedronate, raloxifene and teriparatide for the prevention and treatment of postmenopausal osteoporosis.

By Stevenson M, Lloyd Jones M, De Nigris E, Brewer N, Davis S, Oakley J.

No. 23

A systematic review to examine the impact of psycho-educational interventions on health outcomes and costs in adults and children with difficult asthma.

By Smith JR, Mugford M, Holland R, Candy B, Noble MJ, Harrison BDW, et al.

No. 24

An evaluation of the costs, effectiveness and quality of renal replacement therapy provision in renal satellite units in England and Wales.

By Roderick P, Nicholson T, Armitage A, Mehta R, Mullee M, Gerard K, et al.

No. 25

Imatinib for the treatment of patients with unresectable and/or metastatic gastrointestinal stromal tumours: systematic review and economic evaluation.

By Wilson J, Connock M, Song F, Yao G, Fry-Smith A, Raftery J, et al.

No. 26

Indirect comparisons of competing interventions.

By Glenny AM, Altman DG, Song F, Sakarovitch C, Deeks JJ, D'Amico R, et al.

No. 27

Cost-effectiveness of alternative strategies for the initial medical management of non-ST elevation acute coronary syndrome: systematic review and decision-analytical modelling.

By Robinson M, Palmer S, Sculpher M, Philips Z, Ginnelly L, Bowens A, et al. 
No. 28

Outcomes of electrically stimulated gracilis neosphincter surgery.

By Tillin T, Chambers M, Feldman R.

No. 29

The effectiveness and cost-effectiveness of pimecrolimus and tacrolimus for atopic eczema: a systematic review and economic evaluation.

By Garside R, Stein K, Castelnuovo E, Pitt M, Ashcroft D, Dimmock P, et al.

No. 30

Systematic review on urine albumin testing for early detection of diabetic complications.

By Newman DJ, Mattock MB, Dawnay ABS, Kerry S, McGuire A, Yaqoob M, et al.

No. 31

Randomised controlled trial of the costeffectiveness of water-based therapy for lower limb osteoarthritis.

By Cochrane T, Davey RC, Matthes Edwards SM.

No. 32

Longer term clinical and economic benefits of offering acupuncture care to patients with chronic low back pain.

By Thomas KJ, MacPherson $\mathrm{H}$, Ratcliffe J, Thorpe L, Brazier J, Campbell M, et al.

No. 33

Cost-effectiveness and safety of epidural steroids in the management of sciatica.

By Price C, Arden N, Coglan L, Rogers P.

No. 34

The British Rheumatoid Outcome Study Group (BROSG) randomised controlled trial to compare the effectiveness and cost-effectiveness of aggressive versus symptomatic therapy in established rheumatoid arthritis.

By Symmons D, Tricker K, Roberts C, Davies L, Dawes P, Scott DL.

\section{No. 35}

Conceptual framework and systematic review of the effects of participants' and professionals' preferences in randomised controlled trials.

By King M, Nazareth I, Lampe F, Bower P, Chandler M, Morou M, et al.

No. 36

The clinical and cost-effectiveness of implantable cardioverter defibrillators: a systematic review.

By Bryant J, Brodin H, Loveman E, Payne E, Clegg A.

No. 37

A trial of problem-solving by community mental health nurses for anxiety, depression and life difficulties among general practice patients. The CPN-GP study.

By Kendrick T, Simons L, Mynors-Wallis L, Gray A, Lathlean J, Pickering R, et al.
No. 38

The causes and effects of sociodemographic exclusions from clinical trials.

By Bartlett C, Doyal L, Ebrahim S, Davey P, Bachmann M, Egger M, et al.

\section{No. 39}

Is hydrotherapy cost-effective? A randomised controlled trial of combined hydrotherapy programmes compared with physiotherapy land techniques in children with juvenile idiopathic arthritis.

By Epps H, Ginnelly L, Utley M, Southwood T, Gallivan S, Sculpher M, et al.

\section{No. 40}

A randomised controlled trial and costeffectiveness study of systematic screening (targeted and total population screening) versus routine practice for the detection of atrial fibrillation in people aged 65 and over. The SAFE study.

By Hobbs FDR, Fitzmaurice DA, Mant J, Murray E, Jowett S, Bryan S, et al.

\section{No. 41}

Displaced intracapsular hip fractures in fit, older people: a randomised comparison of reduction and fixation, bipolar hemiarthroplasty and total hip arthroplasty.

By Keating JF, Grant A, Masson M, Scott NW, Forbes JF.

\section{No. 42}

Long-term outcome of cognitive behaviour therapy clinical trials in central Scotland.

By Durham RC, Chambers JA, Power KG, Sharp DM, Macdonald RR, Major KA, et al.

\section{No. 43}

The effectiveness and cost-effectiveness of dual-chamber pacemakers compared with single-chamber pacemakers for bradycardia due to atrioventricular block or sick sinus syndrome: systematic review and economic evaluation.

By Castelnuovo E, Stein K, Pitt M, Garside R, Payne E.

\section{No. 44}

Newborn screening for congenital heart defects: a systematic review and cost-effectiveness analysis.

By Knowles R, Griebsch I, Dezateux C, Brown J, Bull C, Wren C.

\section{No. 45}

The clinical and cost-effectiveness of left ventricular assist devices for end-stage heart failure: a systematic review and economic evaluation.

By Clegg AJ, Scott DA, Loveman E, Colquitt J, Hutchinson J, Royle P, et al.
No. 46

The effectiveness of the Heidelberg

Retina Tomograph and laser diagnostic glaucoma scanning system (GDx) in detecting and monitoring glaucoma

By Kwartz AJ, Henson DB,

Harper RA, Spencer AF,

McLeod D.

No. 47

Clinical and cost-effectiveness of autologous chondrocyte implantation for cartilage defects in knee joints: systematic review and economic evaluation.

By Clar C, Cummins E, McIntyre L, Thomas S, Lamb J, Bain L, et al.

\section{No. 48}

Systematic review of effectiveness of different treatments for childhood retinoblastoma.

By McDaid C, Hartley S, Bagnall A-M, Ritchie G, Light K, Riemsma R.

No. 49

Towards evidence-based guidelines for the prevention of venous thromboembolism: systematic reviews of mechanical methods, oral anticoagulation, dextran and regional anaesthesia as thromboprophylaxis.

By Roderick P, Ferris G, Wilson K, Halls H, Jackson D, Collins R, et al.

No. 50

The effectiveness and cost-effectiveness of parent training/education programmes for the treatment of conduct disorder, including oppositional defiant disorder, in children.

By Dretzke J, Frew E, Davenport C, Barlow J, Stewart-Brown S, Sandercock J, et al.

\section{Volume 10, 2006}

No. 1

The clinical and cost-effectiveness of donepezil, rivastigmine, galantamine and memantine for Alzheimer's disease.

By Loveman E, Green C, Kirby J, Takeda A, Picot J, Payne E, et al.

\section{No. 2}

FOOD: a multicentre randomised trial evaluating feeding policies in patients admitted to hospital with a recent stroke.

By Dennis M, Lewis S, Cranswick G, Forbes J.

No. 3

The clinical effectiveness and costeffectiveness of computed tomography screening for lung cancer: systematic reviews.

By Black C, Bagust A, Boland A Walker S, McLeod C, De Verteuil R, et al. 
No. 4

A systematic review of the effectiveness and cost-effectiveness of neuroimaging assessments used to visualise the seizure focus in people with refractory epilepsy being considered for surgery.

By Whiting P, Gupta R, Burch J, Mujica Mota RE, Wright K, Marson A, et al.

\section{No. 5}

Comparison of conference abstracts and presentations with full-text articles in the health technology assessments of rapidly evolving technologies.

By Dundar Y, Dodd S, Dickson R, Walley T, Haycox A, Williamson PR.

\section{No. 6}

Systematic review and evaluation of methods of assessing urinary incontinence.

By Martin JL, Williams KS, Abrams KR, Turner DA, Sutton AJ, Chapple C, et al.

\section{No. 7}

The clinical effectiveness and costeffectiveness of newer drugs for children with epilepsy. A systematic review.

By Connock M, Frew E, Evans B-W, Bryan S, Cummins C, Fry-Smith A, et al.

\section{No. 8}

Surveillance of Barrett's oesophagus: exploring the uncertainty through systematic review, expert workshop and economic modelling.

By Garside R, Pitt M, Somerville M, Stein K, Price A, Gilbert N.

\section{No. 9}

Topotecan, pegylated liposomal doxorubicin hydrochloride and paclitaxel for second-line or subsequent treatment of advanced ovarian cancer: a systematic review and economic evaluation.

By Main C, Bojke L, Griffin S, Norman G, Barbieri M, Mather L, et al.

No. 10

Evaluation of molecular techniques in prediction and diagnosis of cytomegalovirus disease in immunocompromised patients.

By Szczepura A, Westmoreland D, Vinogradova Y, Fox J, Clark M.

No. 11

Screening for thrombophilia in high-risk situations: systematic review and costeffectiveness analysis. The Thrombosis: Risk and Economic Assessment of Thrombophilia Screening (TREATS) study.

By Wu O, Robertson L, Twaddle S,

Lowe GDO, Clark P, Greaves M, et al.

\section{No. 12}

A series of systematic reviews to inform a decision analysis for sampling and treating infected diabetic foot

ulcers.

By Nelson EA, O'Meara S, Craig D, Iglesias C, Golder S, Dalton J, et al.

\section{No. 13}

Randomised clinical trial, observational study and assessment of cost-

effectiveness of the treatment of varicose veins (REACTIV trial).

By Michaels JA, Campbell WB,

Brazier JE, MacIntyre JB, Palfreyman SJ, Ratcliffe J, et al.

\section{No. 14}

The cost-effectiveness of screening for oral cancer in primary care.

By Speight PM, Palmer S, Moles DR, Downer MC, Smith DH, Henriksson M et al.

\section{No. 15}

Measurement of the clinical and costeffectiveness of non-invasive diagnostic testing strategies for deep vein thrombosis.

By Goodacre S, Sampson F, Stevenson M, Wailoo A, Sutton A, Thomas S, et al.

\section{No. 16}

Systematic review of the effectiveness and cost-effectiveness of HealOzone ${ }^{\circledR}$ for the treatment of occlusal pit/fissure caries and root caries.

By Brazzelli M, McKenzie L, Fielding S, Fraser C, Clarkson J, Kilonzo M, et al.

\section{No. 17}

Randomised controlled trials of conventional antipsychotic versus new atypical drugs, and new atypical drugs versus clozapine, in people with schizophrenia responding poorly to, or intolerant of, current drug treatment.

By Lewis SW, Davies L, Jones PB, Barnes TRE, Murray RM, Kerwin R, et al.

\section{No. 18}

Diagnostic tests and algorithms used in the investigation of haematuria: systematic reviews and economic evaluation.

By Rodgers M, Nixon J, Hempel S, Aho T, Kelly J, Neal D, et al.

\section{No. 19}

Cognitive behavioural therapy in addition to antispasmodic therapy for irritable bowel syndrome in primary care: randomised controlled trial.

By Kennedy TM, Chalder T, McCrone P, Darnley S, Knapp M, Jones RH, et al.
No. 20

A systematic review of the clinical effectiveness and cost-effectiveness of enzyme replacement therapies for

Fabry's disease and mucopolysaccharidosis type 1 .

By Connock M, Juarez-Garcia A, Frew E, Mans A, Dretzke J, Fry-Smith A, et al.

No. 21

Health benefits of antiviral therapy for mild chronic hepatitis $\mathrm{C}$ : randomised controlled trial and economic evaluation.

By Wright M, Grieve R, Roberts J, Main J, Thomas HC on behalf of the UK Mild Hepatitis C Trial Investigators.

\section{No. 22}

Pressure relieving support surfaces: a randomised evaluation.

By Nixon J, Nelson EA, Cranny G, Iglesias CP, Hawkins K, Cullum NA, et al.

No. 23

A systematic review and economic model of the effectiveness and costeffectiveness of methylphenidate, dexamfetamine and atomoxetine for the treatment of attention deficit hyperactivity disorder in children and adolescents.

By King S, Griffin S, Hodges Z, Weatherly H, Asseburg C, Richardson G, et al.

No. 24

The clinical effectiveness and costeffectiveness of enzyme replacement therapy for Gaucher's disease:

a systematic review.

By Connock M, Burls A, Frew E, Fry-Smith A, Juarez-Garcia A McCabe C, et al.

No. 25

Effectiveness and cost-effectiveness of salicylic acid and cryotherapy for cutaneous warts. An economic decision model.

By Thomas KS, Keogh-Brown MR, Chalmers JR, Fordham RJ, Holland RC, Armstrong SJ, et al.

No. 26

A systematic literature review of the effectiveness of non-pharmacological interventions to prevent wandering in dementia and evaluation of the ethical implications and acceptability of their use.

By Robinson L, Hutchings D, Corner L, Beyer F, Dickinson H, Vanoli A, et al.

\section{No. 27}

A review of the evidence on the effects and costs of implantable cardioverter defibrillator therapy in different patient groups, and modelling of costeffectiveness and cost-utility for these groups in a UK context.

By Buxton M, Caine N, Chase D, Connelly D, Grace A, Jackson C, et al. 
No. 28

Adefovir dipivoxil and pegylated interferon alfa-2a for the treatment of chronic hepatitis B: a systematic review and economic evaluation.

By Shepherd J, Jones J, Takeda A, Davidson P, Price A.

No. 29

An evaluation of the clinical and costeffectiveness of pulmonary artery catheters in patient management in intensive care: a systematic review and a randomised controlled trial.

By Harvey S, Stevens K, Harrison D, Young D, Brampton W, McCabe C, et al.

\section{No. 30}

Accurate, practical and cost-effective assessment of carotid stenosis in the UK.

By Wardlaw JM, Chappell FM, Stevenson M, De Nigris E, Thomas S, Gillard J, et al.

No. 31

Etanercept and infliximab for the treatment of psoriatic arthritis: a systematic review and economic evaluation.

By Woolacott N, Bravo Vergel Y, Hawkins N, Kainth A, Khadjesari Z, Misso K, et al.

No. 32

The cost-effectiveness of testing for hepatitis $\mathrm{C}$ in former injecting drug users.

By Castelnuovo E, Thompson-Coon J, Pitt M, Cramp M, Siebert U, Price A, et al.

\section{No. 33}

Computerised cognitive behaviour therapy for depression and anxiety update: a systematic review and economic evaluation.

By Kaltenthaler E, Brazier J, De Nigris E, Tumur I, Ferriter M, Beverley C, et al.

No. 34

Cost-effectiveness of using prognostic information to select women with breast cancer for adjuvant systemic therapy.

By Williams C, Brunskill S, Altman D, Briggs A, Campbell H, Clarke M, et al.

No. 35

Psychological therapies including dialectical behaviour therapy for borderline personality disorder: a systematic review and preliminary economic evaluation.

By Brazier J, Tumur I, Holmes M, Ferriter M, Parry G, Dent-Brown K, et al.

No. 36

Clinical effectiveness and costeffectiveness of tests for the diagnosis and investigation of urinary tract infection in children: a systematic review and economic model.

By Whiting P, Westwood M, Bojke L, Palmer S, Richardson G, Cooper J, et al.

\section{No. 37}

Cognitive behavioural therapy in chronic fatigue syndrome: a randomised controlled trial of an outpatient group programme.

By O'Dowd H, Gladwell P, Rogers CA, Hollinghurst S, Gregory A.

No. 38

A comparison of the cost-effectiveness of five strategies for the prevention of nonsteroidal anti-inflammatory drug-induced gastrointestinal toxicity: a systematic review with economic modelling.

By Brown TJ, Hooper L, Elliott RA, Payne K, Webb R, Roberts C, et al.

\section{No. 39}

The effectiveness and cost-effectiveness of computed tomography screening for coronary artery disease: systematic review.

By Waugh N, Black C, Walker S, McIntyre L, Cummins E, Hillis G.

No. 40

What are the clinical outcome and costeffectiveness of endoscopy undertaken by nurses when compared with doctors? A Multi-Institution Nurse Endoscopy Trial (MINuET).

By Williams J, Russell I, Durai D, Cheung W-Y, Farrin A, Bloor K, et al.

\section{No. 41}

The clinical and cost-effectiveness of oxaliplatin and capecitabine for the adjuvant treatment of colon cancer: systematic review and economic evaluation.

By Pandor A, Eggington S, Paisley S, Tappenden P, Sutcliffe P.

\section{No. 42}

A systematic review of the effectiveness of adalimumab, etanercept and infliximab for the treatment of rheumatoid arthritis in adults and an economic evaluation of their costeffectiveness.

By Chen Y-F, Jobanputra P, Barton P, Jowett S, Bryan S, Clark W, et al.

\section{No. 43}

Telemedicine in dermatology:

a randomised controlled trial.

By Bowns IR, Collins K, Walters SJ, McDonagh AJG.

\section{No. 44}

Cost-effectiveness of cell salvage and alternative methods of minimising perioperative allogeneic blood transfusion: a systematic review and economic model.

By Davies L, Brown TJ, Haynes S, Payne K, Elliott RA, McCollum C.

\section{No. 45}

Clinical effectiveness and costeffectiveness of laparoscopic surgery for colorectal cancer: systematic reviews and economic evaluation.

By Murray A, Lourenco T,

de Verteuil R, Hernandez R, Fraser C, McKinley A, et al.
No. 46

Etanercept and efalizumab for the treatment of psoriasis: a systematic review.

By Woolacott N, Hawkins N, Mason A, Kainth A, Khadjesari Z, Bravo Vergel Y, et al.

No. 47

Systematic reviews of clinical decision tools for acute abdominal pain.

By Liu JLY, Wyatt JC, Deeks JJ, Clamp S, Keen J, Verde P, et al.

\section{No. 48}

Evaluation of the ventricular assist device programme in the UK.

By Sharples L, Buxton M, Caine N, Cafferty F, Demiris N, Dyer M, et al.

\section{No. 49}

A systematic review and economic model of the clinical and costeffectiveness of immunosuppressive therapy for renal transplantation in children.

By Yao G, Albon E, Adi Y, Milford D, Bayliss S, Ready A, et al.

No. 50

Amniocentesis results: investigation of anxiety. The ARIA trial.

By Hewison J, Nixon J, Fountain J, Cocks K, Jones C, Mason G, et al.

\section{Volume I I, 2007}

No. 1

Pemetrexed disodium for the treatment of malignant pleural mesothelioma: a systematic review and economic evaluation.

By Dundar Y, Bagust A, Dickson R, Dodd S, Green J, Haycox A, et al.

\section{No. 2}

A systematic review and economic model of the clinical effectiveness and cost-effectiveness of docetaxel in combination with prednisone or prednisolone for the treatment of hormone-refractory metastatic prostate cancer.

By Collins R, Fenwick E, Trowman R, Perard R, Norman G, Light K, et al.

No. 3

A systematic review of rapid diagnostic tests for the detection of tuberculosis infection.

By Dinnes J, Deeks J, Kunst H, Gibson A, Cummins E, Waugh N, et al.

No. 4

The clinical effectiveness and costeffectiveness of strontium ranelate for the prevention of osteoporotic fragility fractures in postmenopausal women.

By Stevenson M, Davis S, Lloyd-Jones M, Beverley C. 
No. 5

A systematic review of quantitative and qualitative research on the role and effectiveness of written information available to patients about individual medicines.

By Raynor DK, Blenkinsopp A, Knapp P, Grime J, Nicolson DJ, Pollock K, et al.

No. 6

Oral naltrexone as a treatment for relapse prevention in formerly opioid-dependent drug users: a systematic review and economic evaluation.

By Adi Y, Juarez-Garcia A, Wang D, Jowett S, Frew E, Day E, et al.

No. 7

Glucocorticoid-induced osteoporosis: a systematic review and cost-utility analysis.

By Kanis JA, Stevenson M, McCloskey EV, Davis S, Lloyd-Jones M.

\section{No. 8}

Epidemiological, social, diagnostic and economic evaluation of population screening for genital chlamydial infection.

By Low N, McCarthy A, Macleod J, Salisbury C, Campbell R, Roberts TE, et al.

\section{No. 9}

Methadone and buprenorphine for the management of opioid dependence: a systematic review and economic evaluation.

By Connock M, Juarez-Garcia A, Jowett S, Frew E, Liu Z, Taylor RJ, et al.

\section{No. 10}

Exercise Evaluation Randomised Trial (EXERT): a randomised trial comparing GP referral for leisure centre-based exercise, community-based walking and advice only.

By Isaacs AJ, Critchley JA, See Tai S, Buckingham K, Westley D, Harridge SDR, et al.

\section{No. 11}

Interferon alfa (pegylated and non-pegylated) and ribavirin for the treatment of mild chronic hepatitis C: a systematic review and economic evaluation.

By Shepherd J, Jones J, Hartwell D, Davidson P, Price A, Waugh N.

\section{No. 12}

Systematic review and economic evaluation of bevacizumab and cetuximab for the treatment of metastatic colorectal cancer

By Tappenden P, Jones R, Paisley S, Carroll C.

\section{No. 13}

A systematic review and economic evaluation of epoetin alfa, epoetin beta and darbepoetin alfa in anaemia associated with cancer, especially that attributable to cancer treatment.

By Wilson J, Yao GL, Raftery J,

Bohlius J, Brunskill S, Sandercock J, et al.

\section{No. 14}

A systematic review and economic evaluation of statins for the prevention of coronary events.

By Ward S, Lloyd Jones M, Pandor A, Holmes M, Ara R, Ryan A, et al.

\section{No. 15}

A systematic review of the effectiveness and cost-effectiveness of different models of community-based respite care for frail older people and their carers.

By Mason A, Weatherly H, Spilsbury K, Arksey H, Golder S, Adamson J, et al.

\section{No. 16}

Additional therapy for young children with spastic cerebral palsy:

a randomised controlled trial.

By Weindling AM, Cunningham CC, Glenn SM, Edwards RT, Reeves DJ.

\section{No. 17}

Screening for type 2 diabetes: literature review and economic modelling.

By Waugh N, Scotland G,

McNamee P, Gillett M, Brennan A, Goyder E, et al.

\section{No. 18}

The effectiveness and cost-effectiveness of cinacalcet for secondary hyperparathyroidism in end-stage renal disease patients on dialysis: a systematic review and economic evaluation.

By Garside R, Pitt M, Anderson R, Mealing S, Roome C, Snaith A, et al.

\section{No. 19}

The clinical effectiveness and cost-effectiveness of gemcitabine for metastatic breast cancer: a systematic review and economic evaluation.

By Takeda AL, Jones J, Loveman E, Tan SC, Clegg AJ.

\section{No. 20}

A systematic review of duplex ultrasound, magnetic resonance angiography and computed tomography angiography for the diagnosis and assessment of symptomatic, lower limb peripheral arterial disease.

By Collins R, Cranny G, Burch J, Aguiar-Ibáñez R, Craig D, Wright K, et al.
No. 21

The clinical effectiveness and costeffectiveness of treatments for children with idiopathic steroid-resistant nephrotic syndrome: a systematic review.

By Colquitt JL, Kirby J, Green C, Cooper K, Trompeter RS.

No. 22

A systematic review of the routine monitoring of growth in children of primary school age to identify growth-related conditions.

By Fayter D, Nixon J, Hartley S, Rithalia A, Butler G, Rudolf M, et al.

No. 23

Systematic review of the effectiveness of preventing and treating Staphylococcus aureus carriage in reducing peritoneal catheter-related infections.

By McCormack K, Rabindranath K, Kilonzo M, Vale L, Fraser C, McIntyre L, et al.

\section{No. 24}

The clinical effectiveness and cost of repetitive transcranial magnetic stimulation versus electroconvulsive therapy in severe depression: a multicentre pragmatic randomised controlled trial and economic analysis.

By McLoughlin DM, Mogg A, Eranti S, Pluck G, Purvis R, Edwards D, et al.

\section{No. 25}

A randomised controlled trial and economic evaluation of direct versus indirect and individual versus group modes of speech and language therapy for children with primary language impairment.

By Boyle J, McCartney E, Forbes J, O'Hare A.

\section{No. 26}

Hormonal therapies for early breast cancer: systematic review and economic evaluation.

By Hind D, Ward S, De Nigris E, Simpson E, Carroll C, Wyld L.

No. 27

Cardioprotection against the toxic effects of anthracyclines given to children with cancer: a systematic review.

By Bryant J, Picot J, Levitt G, Sullivan I, Baxter L, Clegg A.

No. 28

Adalimumab, etanercept and infliximab for the treatment of ankylosing spondylitis: a systematic review and economic evaluation.

By McLeod C, Bagust A, Boland A, Dagenais P, Dickson R, Dundar Y, et al. 
No. 29

Prenatal screening and treatment strategies to prevent group B streptococcal and other bacterial infections in early infancy: cost-effectiveness and expected value of information analyses.

By Colbourn T, Asseburg C, Bojke L, Philips Z, Claxton K, Ades AE, et al.

\section{No. 30}

Clinical effectiveness and costeffectiveness of bone morphogenetic proteins in the non-healing of fractures and spinal fusion: a systematic review.

By Garrison KR, Donell S, Ryder J, Shemilt I, Mugford M, Harvey I, et al.

\section{No. 31}

A randomised controlled trial of postoperative radiotherapy following breast-conserving surgery in a minimum-risk older population. The PRIME trial.

By Prescott RJ, Kunkler IH, Williams LJ, King CC, Jack W, van der Pol M, et al.

No. 32

Current practice, accuracy, effectiveness and cost-effectiveness of the school entry hearing screen.

By Bamford J, Fortnum H, Bristow K, Smith J, Vamvakas G, Davies L, et al.

No. 33

The clinical effectiveness and cost-effectiveness of inhaled insulin in diabetes mellitus: a systematic review and economic evaluation.

By Black C, Cummins E, Royle P, Philip S, Waugh N.

No. 34

Surveillance of cirrhosis for hepatocellular carcinoma: systematic review and economic analysis.

By Thompson Coon J, Rogers G, Hewson P, Wright D, Anderson R, Cramp M, et al.

No. 35

The Birmingham Rehabilitation Uptake Maximisation Study (BRUM). Homebased compared with hospital-based cardiac rehabilitation in a multi-ethnic population: cost-effectiveness and patient adherence.

By Jolly K, Taylor R, Lip GYH, Greenfield S, Raftery J, Mant J, et al.

No. 36

A systematic review of the clinical, public health and cost-effectiveness of rapid diagnostic tests for the detection and identification of bacterial intestinal pathogens in faeces and food.

By Abubakar I, Irvine L, Aldus CF, Wyatt GM, Fordham R, Schelenz S, et al.
No. 37

A randomised controlled trial

examining the longer-term outcomes of standard versus new antiepileptic drugs. The SANAD trial.

By Marson AG, Appleton R, Baker GA, Chadwick DW, Doughty J, Eaton B, et al.

No. 38

Clinical effectiveness and cost-

effectiveness of different models of managing long-term oral anticoagulation therapy: a systematic review and economic modelling.

By Connock M, Stevens C Fry-Smith A, Jowett S, Fitzmaurice D, Moore D, et al.

No. 39

A systematic review and economic model of the clinical effectiveness and cost-effectiveness of interventions for preventing relapse in people with bipolar disorder.

By Soares-Weiser K, Bravo Vergel Y, Beynon S, Dunn G, Barbieri M, Duffy S, et al.

\section{No. 40}

Taxanes for the adjuvant treatment of early breast cancer: systematic review and economic evaluation.

By Ward S, Simpson E, Davis S, Hind D, Rees A, Wilkinson A.

\section{No. 41}

The clinical effectiveness and costeffectiveness of screening for open angle glaucoma: a systematic review and economic evaluation.

By Burr JM, Mowatt G, Hernández R, Siddiqui MAR, Cook J, Lourenco T, et al.

No. 42

Acceptability, benefit and costs of early screening for hearing disability: a study of potential screening tests and models.

By Davis A, Smith P, Ferguson M, Stephens D, Gianopoulos I.

No. 43

Contamination in trials of educational interventions.

By Keogh-Brown MR, Bachmann MO, Shepstone L, Hewitt C, Howe A, Ramsay CR, et al.

No. 44

Overview of the clinical effectiveness of positron emission tomography imaging in selected cancers.

By Facey K, Bradbury I, Laking G, Payne E.

\section{No. 45}

The effectiveness and cost-effectiveness of carmustine implants and temozolomide for the treatment of newly diagnosed high-grade glioma: a systematic review and economic evaluation.

By Garside R, Pitt M, Anderson R, Rogers G, Dyer M, Mealing S, et al.
No. 46

Drug-eluting stents: a systematic review and economic evaluation.

By Hill RA, Boland A, Dickson R, Dündar Y, Haycox A, McLeod C, et al.

\section{No. 47}

The clinical effectiveness and costeffectiveness of cardiac resynchronisation (biventricular pacing) for heart failure: systematic review and economic model.

By Fox M, Mealing S, Anderson R,

Dean J, Stein K, Price A, et al.

No. 48

Recruitment to randomised trials: strategies for trial enrolment and participation study. The STEPS study.

By Campbell MK, Snowdon C, Francis D, Elbourne D, McDonald AM, Knight R, et al.

No. 49

Cost-effectiveness of functional cardiac testing in the diagnosis and management of coronary artery disease: a randomised controlled trial.

The CECaT trial.

By Sharples L, Hughes V, Crean A, Dyer M, Buxton M, Goldsmith K, et al.

No. 50

Evaluation of diagnostic tests when there is no gold standard. A review of methods.

By Rutjes AWS, Reitsma JB,

Coomarasamy A, Khan KS,

Bossuyt PMM.

No. 51

Systematic reviews of the clinical effectiveness and cost-effectiveness of proton pump inhibitors in acute upper gastrointestinal bleeding.

By Leontiadis GI, Sreedharan A, Dorward S, Barton P, Delaney B,

Howden CW, et al.

No. 52

A review and critique of modelling in prioritising and designing screening programmes.

By Karnon J, Goyder E, Tappenden P, McPhie S, Towers I, Brazier J, et al.

No. 53

An assessment of the impact of the NHS Health Technology Assessment Programme.

By Hanney S, Buxton M, Green C, Coulson D, Raftery J.

\section{Volume I2, 2008}

No. 1

A systematic review and economic mode of switching from non-glycopeptide to glycopeptide antibiotic prophylaxis for surgery.

By Cranny G, Elliott R, Weatherly H, Chambers D, Hawkins N, Myers L, et al. 



\section{Health Technology Assessment Programme}

\section{Members}

\section{Chair,}

Professor Tom Walley,

Director, NHS HTA Programme,

Department of Pharmacology \&

Therapeutics,

University of Liverpool

\section{Director,}

Professor Tom Walley,

Director, NHS HTA Programme,

Department of Pharmacology \&

Therapeutics,

University of Liverpool

\section{Deputy Director,}

Professor Jon Nicholl,

Director, Medical Care Research

Unit, University of Sheffield,

School of Health and Related

Research

\section{Prioritisation Strategy Group}

Professor Bruce Campbell,

Consultant Vascular \& General

Surgeon, Royal Devon \& Exeter

Hospital

Professor Robin E Ferner,

Consultant Physician and Director, West Midlands Centre

for Adverse Drug Reactions,

City Hospital NHS Trust,

Birmingham
Dr Edmund Jessop, Medical

Adviser, National Specialist,

Commissioning Advisory Group

(NSCAG), Department of

Health, London

Professor Jon Nicholl, Director,

Medical Care Research Unit,

University of Sheffield,

School of Health and

Related Research
Dr Ron Zimmern, Director, Public Health Genetics Unit, Strangeways Research Laboratories, Cambridge

\section{HTA Commissioning Board}

Members

Programme Director,

Professor Tom Walley,

Director, NHS HTA Programme,

Department of Pharmacology \&

Therapeutics,

University of Liverpool

Chair,

Professor Jon Nicholl,

Director, Medical Care Research

Unit, University of Sheffield,

School of Health and Related

Research

Deputy Chair,

Dr Andrew Farmer,

University Lecturer in General

Practice, Department of

Primary Health Care,

University of Oxford
Professor Deborah Ashby,

Professor of Medical Statistics,

Department of Environmental

and Preventative Medicine,

Queen Mary University of

London

Professor Ann Bowling,

Professor of Health Services

Research, Primary Care and

Population Studies,

University College London

Professor John Cairns,

Professor of Health Economics,

Public Health Policy,

London School of Hygiene

and Tropical Medicine,

London

Professor Nicky Cullum,

Director of Centre for Evidence

Based Nursing, Department of

Health Sciences, University of

Dr Jeffrey Aronson,

Reader in Clinical

Pharmacology, Department of

Clinical Pharmacology,

Radcliffe Infirmary, Oxford

\section{York}

Professor Jon Deeks,

Professor of Health Statistics,

University of Birmingham
Professor Jenny Donovan,

Professor of Social Medicine,

Department of Social Medicine,

University of Bristol

Professor Freddie Hamdy,

Professor of Urology,

University of Sheffield

Professor Allan House,

Professor of Liaison Psychiatry,

University of Leeds

Professor Sallie Lamb, Director, Warwick Clinical Trials Unit, University of Warwick

Professor Stuart Logan, Director of Health \& Social Care Research, The Peninsula Medical School, Universities of Exeter \& Plymouth

Professor Miranda Mugford, Professor of Health Economics, University of East Anglia

Dr Linda Patterson,

Consultant Physician,

Department of Medicine,

Burnley General Hospital
Professor Ian Roberts, Professor of Epidemiology \& Public Health, Intervention Research Unit, London School of Hygiene and Tropical Medicine

Professor Mark Sculpher, Professor of Health Economics, Centre for Health Economics, Institute for Research in the Social Services,

University of York

Professor Kate Thomas, Professor of Complementary and Alternative Medicine, University of Leeds

Professor David John Torgerson, Director of York Trial Unit, Department of Health Sciences, University of York

Professor Hywel Williams,

Professor of

Dermato-Epidemiology,

University of Nottingham 


\section{Diagnostic Technologies \& Screening Panel}

Members

\section{Chair,}

Dr Ron Zimmern, Director of the Public Health Genetics Unit, Strangeways Research Laboratories, Cambridge

Ms Norma Armston, Freelance Consumer Advocate, Bolton

Professor Max Bachmann, Professor of Health Care Interfaces, Department of Health Policy and Practice, University of East Anglia

Professor Rudy Bilous Professor of Clinical Medicine \& Consultant Physician,

The Academic Centre,

South Tees Hospitals NHS Trust

Ms Dea Birkett, Service User Representative, London
Dr Paul Cockcroft, Consultant Medical Microbiologist and Clinical Director of Pathology, Department of Clinical Microbiology, St Mary's Hospital, Portsmouth

Professor Adrian K Dixon, Professor of Radiology, University Department of Radiology, University of Cambridge Clinical School

Dr David Elliman, Consultant in Community Child Health, Islington PCT \& Great Ormond Street Hospital, London

Professor Glyn Elwyn, Research Chair, Centre for Health Sciences Research, Cardiff University, Department of General Practice, Cardiff

Professor Paul Glasziou, Director, Centre for Evidence-Based Practice, University of Oxford
Dr Jennifer J Kurinczuk, Consultant Clinical Epidemiologist, National Perinatal Epidemiology Unit, Oxford

Dr Susanne M Ludgate, Clinical Director, Medicines \& Healthcare Products Regulatory Agency, London

Mr Stephen Pilling, Director, Centre for Outcomes, Research \& Effectiveness, Joint Director, National Collaborating Centre for Mental Health, University College London

Mrs Una Rennard,

Service User Representative,

Oxford

Dr Phil Shackley, Senior Lecturer in Health Economics, Academic Vascular Unit, University of Sheffield
Dr Margaret Somerville, Director of Public Health Learning, Peninsula Medical School, University of Plymouth

Dr Graham Taylor, Scientific Director \& Senior Lecturer, Regional DNA Laboratory, The Leeds Teaching Hospitals

Professor Lindsay Wilson Turnbull, Scientific Director, Centre for MR Investigations \& YCR Professor of Radiology, University of Hul

Professor Martin J Whittle, Clinical Co-director, National Co-ordinating Centre for Women's and Childhealth

Dr Dennis Wright, Consultant Biochemist \& Clinical Director, The North West London Hospitals NHS Trust, Middlesex

\section{Pharmaceuticals Panel}

\section{Chair,}

Professor Robin Ferner,

Consultant Physician and Director, West Midlands Centre for Adverse Drug Reactions, City Hospital NHS Trust, Birmingham

Ms Anne Baileff, Consultant Nurse in First Contact Care, Southampton City Primary Care Trust, University of Southampton
Professor Imti Choonara, Professor in Child Health, Academic Division of Child Health, University of Nottingham

Professor John Geddes, Professor of Epidemiological Psychiatry, University of Oxford

Mrs Barbara Greggains, Non-Executive Director, Greggains Management Ltd

Dr Bill Gutteridge, Medical Adviser, National Specialist Commissioning Advisory Group (NSCAG), London

Mrs Sharon Hart, Consultant Pharmaceutical Adviser, Reading
Dr Jonathan Karnon, Senior Research Fellow, Health Economics and Decision Science, University of Sheffield

Dr Yoon Loke, Senior Lecturer in Clinical Pharmacology, University of East Anglia

Ms Barbara Meredith, Lay Member, Epsom

Dr Andrew Prentice, Senior Lecturer and Consultant Obstetrician \& Gynaecologist, Department of Obstetrics \& Gynaecology, University of Cambridge

Dr Frances Rotblat, CPMP Delegate, Medicines \& Healthcare Products Regulatory Agency, London

\section{Dr Martin Shelly, General Practitioner, Leeds}

Mrs Katrina Simister, Assistant Director New Medicines, National Prescribing Centre, Liverpool

Dr Richard Tiner, Medical Director, Medical Department, Association of the British Pharmaceutical Industry, London 


\section{Therapeutic Procedures Panel}

Members

\section{Chair,}

Professor Bruce Campbell,

Consultant Vascular and General Surgeon, Department of Surgery, Royal Devon \& Exeter Hospital

Dr Mahmood Adil, Deputy Regional Director of Public Health, Department of Health, Manchester

Dr Aileen Clarke, Consultant in Public Health, Public Health Resource Unit, Oxford
Professor Matthew Cooke,

Professor of Emergency

Medicine, Warwick Emergency

Care and Rehabilitation,

University of Warwick

Mr Mark Emberton, Senior

Lecturer in Oncological

Urology, Institute of Urology,

University College Hospital

Professor Paul Gregg,

Professor of Orthopaedic

Surgical Science, Department of

General Practice and Primary

Care, South Tees Hospital NHS

Trust, Middlesbrough

Ms Maryann L Hardy,

Lecturer, Division of

Radiography, University of

Bradford
Dr Simon de Lusignan, Senior Lecturer, Primary Care Informatics, Department of Community Health Sciences, St George's Hospital Medical School, London

Dr Peter Martin, Consultant Neurologist, Addenbrooke's Hospital, Cambridge

Professor Neil McIntosh, Edward Clark Professor of Child Life \& Health, Department of Child Life \& Health, University of Edinburgh

Professor Jim Neilson, Professor of Obstetrics and Gynaecology, Department of Obstetrics and Gynaecology, University of Liverpool
Dr John C Pounsford,

Consultant Physician,

Directorate of Medical Services, North Bristol NHS Trust

Dr Karen Roberts, Nurse Consultant, Queen Elizabeth Hospital, Gateshead

Dr Vimal Sharma, Consultant Psychiatrist/Hon. Senior Lecturer, Mental Health Resource Centre, Cheshire and Wirral Partnership NHS Trust, Wallasey

Professor Scott Weich, Professor of Psychiatry, Division of Health in the Community, University of Warwick

Members

\section{Chair, \\ Dr Edmund Jessop, Medical} Adviser, National Specialist Commissioning Advisory Group (NSCAG), London

Mrs Sheila Clark, Chief Executive, St James's Hospital, Portsmouth

Mr Richard Copeland, Lead Pharmacist: Clinical Economy/Interface, Wansbeck General Hospital, Northumberland
Dr Elizabeth Fellow-Smith, Medical Director, West London Mental Health Trust, Middlesex

Mr Ian Flack, Director PPI Forum Support, Council of Ethnic Minority Voluntary Sector Organisations, Stratford

Dr John Jackson, General Practitioner, Newcastle upon Tyne

Mrs Veronica James, Chief Officer, Horsham District Age Concern, Horsham

Professor Mike Kelly, Director, Centre for Public Health Excellence,

National Institute for Health and Clinical Excellence, London
Professor Yi Mien Koh, Director of Public Health and Medical Director, London NHS (North West London Strategic Health Authority), London

Ms Jeanett Martin, Director of Clinical Leadership \& Quality, Lewisham PCT, London

Dr Chris McCall, General Practitioner, Dorset

Dr David Pencheon, Director, Eastern Region Public Health Observatory, Cambridge

Dr Ken Stein, Senior Clinical Lecturer in Public Health, Director, Peninsula Technology

Assessment Group,

University of Exeter,

Exeter
Dr Carol Tannahill, Director, Glasgow Centre for Population Health, Glasgow

Professor Margaret Thorogood, Professor of Epidemiology, University of Warwick, Coventry

Dr Ewan Wilkinson, Consultant in Public Health, Royal Liverpool University Hospital, Liverpool 


\section{Expert Advisory Network}

\section{Members}

Professor Douglas Altman, Professor of Statistics in Medicine, Centre for Statistics in Medicine, University of Oxford

Professor John Bond, Director, Centre for Health Services Research, University of Newcastle upon Tyne, School of Population \& Health Sciences, Newcastle upon Tyne

Professor Andrew Bradbury, Professor of Vascular Surgery, Solihull Hospital, Birmingham

Mr Shaun Brogan,

Chief Executive, Ridgeway

Primary Care Group, Aylesbury

Mrs Stella Burnside OBE

Chief Executive,

Regulation and Improvement

Authority, Belfast

Ms Tracy Bury,

Project Manager, World

Confederation for Physical

Therapy, London

Professor Iain T Cameron, Professor of Obstetrics and Gynaecology and Head of the School of Medicine, University of Southampton

Dr Christine Clark, Medical Writer \& Consultant Pharmacist, Rossendale

Professor Collette Clifford, Professor of Nursing \& Head of Research, School of Health Sciences, University of Birmingham, Edgbaston, Birmingham

Professor Barry Cookson, Director, Laboratory of Healthcare Associated Infection, Health Protection Agency, London

Dr Carl Counsell, Clinical Senior Lecturer in Neurology, Department of Medicine \& Therapeutics, University of Aberdeen

Professor Howard Cuckle, Professor of Reproductive Epidemiology, Department of Paediatrics, Obstetrics \& Gynaecology, University of Leeds

Dr Katherine Darton, Information Unit, MIND The Mental Health Charity, London
Professor Carol Dezateux, Professor of Paediatric Epidemiology, London

Dr Keith Dodd, Consultant Paediatrician, Derby

Mr John Dunning, Consultant Cardiothoracic Surgeon, Cardiothoracic Surgical Unit, Papworth Hospital NHS Trust, Cambridge

Mr Jonothan Earnshaw, Consultant Vascular Surgeon, Gloucestershire Royal Hospital, Gloucester

Professor Martin Eccles, Professor of Clinical

Effectiveness, Centre for Health Services Research, University of Newcastle upon Tyne

Professor Pam Enderby, Professor of Community Rehabilitation, Institute of General Practice and Primary Care, University of Sheffield

Professor Gene Feder, Professor of Primary Care Research \& Development, Centre for Health Sciences, Barts \& The London Queen Mary's School of Medicine \& Dentistry, London

Mr Leonard R Fenwick,

Chief Executive, Newcastle upon Tyne Hospitals NHS Trust

Mrs Gillian Fletcher,

Antenatal Teacher \& Tutor and President, National Childbirth Trust, Henfield

Professor Jayne Franklyn, Professor of Medicine,

Department of Medicine, University of Birmingham, Queen Elizabeth Hospital,

Edgbaston, Birmingham

Dr Neville Goodman,

Consultant Anaesthetist, Southmead Hospital, Bristol

Professor Robert E Hawkins, CRC Professor and Director of Medical Oncology, Christie CRC Research Centre, Christie Hospital NHS Trust, Manchester

Professor Allen Hutchinson, Director of Public Health \& Deputy Dean of ScHARR, Department of Public Health, University of Sheffield

Professor Peter Jones, Professor of Psychiatry, University of Cambridge, Cambridge
Professor Stan Kave, Cancer Research UK Professor of Medical Oncology, Section of Medicine, Royal Marsden Hospital \& Institute of Cancer Research, Surrey

Dr Duncan Keeley, General Practitioner (Dr Burch \& Ptnrs), The Health Centre, Thame

Dr Donna Lamping, Research Degrees Programme Director \& Reader in Psychology, Health Services Research Unit, London School of Hygiene and Tropical Medicine, London

Mr George Levvy,

Chief Executive, Motor

Neurone Disease Association, Northampton

Professor James Lindesay, Professor of Psychiatry for the Elderly, University of Leicester, Leicester General Hospital

Professor Julian Little, Professor of Human Genome Epidemiology, Department of Epidemiology \& Community Medicine, University of Ottawa

Professor Rajan Madhok, Consultant in Public Health, South Manchester Primary Care Trust, Manchester

Professor Alexander Markham, Director, Molecular Medicine Unit, St James's University Hospital, Leeds

Professor Alistaire McGuire, Professor of Health Economics, London School of Economics

Dr Peter Moore,

Freelance Science Writer, Ashtead

Dr Andrew Mortimore, Public Health Director, Southampton City Primary Care Trust, Southampton

Dr Sue Moss, Associate Director, Cancer Screening Evaluation Unit, Institute of Cancer Research, Sutton

Mrs Julietta Patnick, Director, NHS Cancer Screening Programmes, Sheffield

Professor Robert Peveler, Professor of Liaison Psychiatry, Royal South Hants Hospital, Southampton
Professor Chris Price,

Visiting Professor in Clinica Biochemistry, University of Oxford

Professor William Rosenberg, Professor of Hepatology and Consultant Physician, University of Southampton, Southampton

Professor Peter Sandercock, Professor of Medical Neurology, Department of Clinical Neurosciences, University of Edinburgh

Dr Susan Schonfield, Consultant in Public Health, Hillingdon PCT, Middlesex

Dr Eamonn Sheridan,

Consultant in Clinical Genetics, Genetics Department, St James's University Hospital, Leeds

Professor Sarah Stewart-Brown, Professor of Public Health, University of Warwick, Division of Health in the Community Warwick Medical School, LWMS, Coventry

Professor Ala Szczepura, Professor of Health Service Research, Centre for Health Services Studies, University of Warwick

Dr Ross Taylor, Senior Lecturer, Department of General Practice and Primary Care, University of Aberdeen

Mrs Joan Webster, Consumer member, HTA Expert Advisory Network 



\section{Feedback}

The HTA Programme and the authors would like to know your views about this report.

The Correspondence Page on the HTA website (http://www.hta.ac.uk) is a convenient way to publish your comments. If you prefer, you can send your comments

to the address below, telling us whether you would like us to transfer them to the website.

We look forward to hearing from you.

The National Coordinating Centre for Health Technology Assessment,

Mailpoint 728, Boldrewood,

University of Southampton,

Southampton, SO 16 7PX, UK.

Fax: +44 (0) $2380595639 \quad$ Email: hta@hta.ac.uk

http://www.hta.ac.uk 